\title{
Total Synthesis of Thuggacin cmc-A and Its Structure Determination
}

Tomohiro Tsutsumi ${ }^{\dagger}$, Moe Matsumoto ${ }^{\dagger}$, Hitomi Iwasaki ${ }^{\dagger}$, Kei Tomisawa ${ }^{\dagger}$, Keita Komine ${ }^{\dagger}$, Hayato Fukuda ${ }^{\dagger}$, Jacques Eustache ${ }^{\S}$, Rolf Jansen`, Susumi Hatakeyama ${ }^{\ddagger}$, and Jun Ishihara*†

Graduate School of Biomedical Sciences, Nagasaki University, Nagasaki, 852-8521, Japan. §École Nationale Supérieure de Chimie de Mulhouse, Université de Haute-Alsace, 3 rue A. Werner, 68093 Mulhouse Cedex, France. 'Department Microbial Drugs, Helmholtz Centre for Infection Research, Inhoffenstraße 7, D-38124 Braunschweig, Germany.

${ }^{\ddagger}$ Medical Innovation Center, Nagasaki University, Nagasaki, 852-8521, Japan.

\section{Supporting Information}

Table of Contents

Page

Experimental Procedure and Characterization of Compounds

General

Experiments in Scheme 2

Experiments in Scheme 3

Experiments in Scheme 4

NMR Comparison of Synthetic Thuggacin cmc-A with the Natural Product

References

${ }^{1} \mathrm{H}$ and ${ }^{13} \mathrm{C}$ NMR Spectra 
General. Where appropriate, reactions were performed in flame-dried glassware under argon atmosphere. For reactions that require heating, an oil bath was used as the heat source. All extracts were dried over $\mathrm{MgSO}_{4}$ and concentrated by rotary evaporation below $30{ }^{\circ} \mathrm{C}$ at 25 Torr unless otherwise noted. Commercial reagents and solvents were used as supplied with following exceptions. Dichloromethane $\left(\mathrm{CH}_{2} \mathrm{Cl}_{2}\right)$, hexane, pyridine, toluene and triethylamine $\left(\mathrm{Et}_{3} \mathrm{~N}\right)$ were distilled over $\mathrm{CaH}_{2}$. Methanol $(\mathrm{MeOH})$ was distilled over Na. Thin-layer chromatography (TLC) was performed using precoated silica gel plates $(0.2$ or $0.5 \mathrm{~mm}$ thickness) and silica gel $60 \mathrm{RP}-18$ (0.25 mm thickness). Column chromatography was performed using silica gel (particle size 100-210 $\mu \mathrm{m}$ (regular), 40-50 $\mu \mathrm{m}$ (flash)). Optical rotations were recorded on a digital polarimeter at ambient temperature. Infrared spectra (FTIR) were measured on a Fourier transform infrared spectrometer. ${ }^{1} \mathrm{H}$ NMR $(300,400$ and $500 \mathrm{MHz})$ and ${ }^{13} \mathrm{C} \operatorname{NMR}(75,100$ and $125 \mathrm{MHz})$ spectra were measured using $\mathrm{CDCl}_{3}$ or DMSO- $\mathrm{d}_{6}$ as solvent, and chemical shifts are reported as $\delta$ values in ppm based on solvent peak $\left(\mathrm{CHCl}_{3},{ }^{1} \mathrm{H}: 7.26\right.$ ppm, ${ }^{13} \mathrm{C}: 77.0$ ppm; DMSO-d $\left.6,{ }^{1} \mathrm{H}: 2.50 \mathrm{ppm},{ }^{13} \mathrm{C}: 39.5 \mathrm{ppm}\right)$. Mass spectra (MS) and high-resolution MS (HRMS) were recorded in EI (dual focusing sector field), FAB (dual focusing sector field), or ESI (time of flight [TOF]) mode.

\section{Experiments in Scheme 2}

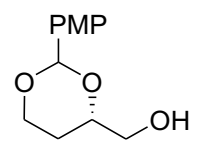

((4S)-2-(4-Methoxybenzyl)-1,3-dioxan-4-yl)methanol (S1): ${ }^{1}$ To an ice-cooled solution of borane dimethylsulfide complex (10.0-10.2 M, 34.0 mL, $358 \mathrm{mmol})$ in THF (149 mL) were added trimethoxyborane $(43.7 \mathrm{~mL}, 392 \mathrm{mmol})$ and a solution of L-malic acid (15 g, $112 \mathrm{mmol})$ in THF $(75 \mathrm{~mL})$, and the mixture was stirred at room temperature for $15 \mathrm{~h}$. The mixture was diluted with methanol $(120 \mathrm{~mL})$ at $0{ }^{\circ} \mathrm{C}$ and concentrated. The residue was diluted with $\mathrm{CH}_{2} \mathrm{Cl}_{2}(40 \mathrm{~mL})$ and methanol $(10 \mathrm{~mL})$, and the mixture was filtered through $\mathrm{SiO}_{2}$ and concentrated. Further dilution of the residue with $\mathrm{CH}_{2} \mathrm{Cl}_{2}(18 \mathrm{~mL})$ and methanol ( $2 \mathrm{~mL})$ and concentration gave triol (11.6 g, $109 \mathrm{mmol}, 97 \%)$.

To an ice-cooled solution of the resulting triol (11.6 g, $109 \mathrm{mmol})$ in $\mathrm{CH}_{2} \mathrm{Cl}_{2}(112 \mathrm{~mL})$ were added $p$-anisaldehyde dimethylacetal $(38 \mathrm{~mL}, 224 \mathrm{mmol})$ and 10-camphorsulfonic acid $(2.6 \mathrm{~g}$, $11.2 \mathrm{mmol}$ ), and the mixture was stirred at room temperature for $42 \mathrm{~h}$. The reaction was quenched with triethylamine $(1.5 \mathrm{~mL}, 10.8 \mathrm{mmol})$ and the mixture was concentrated. The residue was purified by column chromatography $\left(\mathrm{SiO}_{2} 1000 \mathrm{~g}\right.$, hexane/EtOAc $=2: 1$ to $\left.1: 1\right)$ to afford $\mathbf{S 1}\left(18.8 \mathrm{~g}, 83.8 \mathrm{mmol}, 75 \%, 2\right.$ steps) as a colorless oil. $[\alpha]_{\mathrm{D}}{ }^{23}+6.8\left(c \mathrm{1.04}, \mathrm{CHCl}_{3}\right) ;{ }^{1} \mathrm{H}$ NMR (400 MHz, $\left.\mathrm{CDCl}_{3}\right) \delta 7.42(\mathrm{~d}, J=8.6 \mathrm{~Hz}, 2 \mathrm{H}), 6.89(\mathrm{~d}, J=8.6 \mathrm{~Hz}, 2 \mathrm{H}), 5.50(\mathrm{~s}, 1 \mathrm{H})$, $4.30(\mathrm{dd}, J=11.4,5.1 \mathrm{~Hz}, 1 \mathrm{H}), 4.05-3.97$ (m, 2H), 3.80 (s, 3H), 3.75-3.62 (m, 2H), 2.10 (br, 1H), $1.95-1.85(\mathrm{~m}, 1 \mathrm{H}), 1.43(\mathrm{~d}, J=12.4 \mathrm{~Hz}, 1 \mathrm{H}) ;{ }^{13} \mathrm{C} \mathrm{NMR}\left(100 \mathrm{MHz}, \mathrm{CDCl}_{3}\right) \delta 160.0,130.9$, 127.4, 133.6, 101.2, 77.4, 66.5, 65.7, 55.3, 26.7; FT-IR (neat) v 3437, 2858, 1615, 1516, 1391, 
1308, 1251, 1114, 829, 780, $593 \mathrm{~cm}^{-1}$; MS (ESI) $\mathrm{m} / z: 108$ (100), 247 [(M+Na) ${ }^{+}$; HRMS (ESI) calcd for $\mathrm{C}_{12} \mathrm{H}_{16} \mathrm{NaO}_{4}\left[(\mathrm{M}+\mathrm{Na})^{+}\right]$247.0946, found 247.0976.

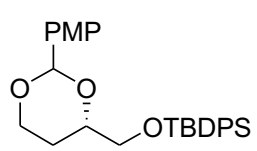

(4S)-2-(4-Methoxybenzyl)-4-tert-butyldiphenylsiloxymethyl-1,3-dioxane (13): To an ice-cooled solution of $\mathbf{S 1}(5.8 \mathrm{~g}, 25.8 \mathrm{mmol})$ in $\mathrm{CH}_{2} \mathrm{Cl}_{2}(129 \mathrm{~mL})$ were added imidazole ( $4.2 \mathrm{~g}, 62 \mathrm{mmol})$ and TBDPSCl $(7.9 \mathrm{~mL}, 31.0 \mathrm{mmol})$, and the mixture was stirred at room temperature for $2 \mathrm{~h}$. The mixture was diluted with EtOAc $(300 \mathrm{~mL})$, washed with saturated $\mathrm{NH}_{4} \mathrm{Cl}(100 \mathrm{~mL})$, saturated $\mathrm{NaHCO}_{3}(100 \mathrm{~mL})$ and brine $(100$ $\mathrm{mL})$, dried, and concentrated. The residue was purified by column chromatography $\left(\mathrm{SiO}_{2} 300\right.$ $\mathrm{g}$, hexane/EtOAc $=10: 1)$ to give $\mathbf{1 3}(12.0 \mathrm{~g}, 25.8 \mathrm{mmol}, 100 \%)$ as a colorless oil. $[\alpha]_{\mathrm{D}}{ }^{24}-0.05$ (c 1.02, $\left.\mathrm{CHCl}_{3}\right) ;{ }^{1} \mathrm{H}$ NMR (400 MHz, $\left.\mathrm{CDCl}_{3}\right) \delta$ 7.72-7.67 (m, 4H), 7.43-7.33 (m, 8H), 6.87 (d, $J=8.5 \mathrm{~Hz}, 2 \mathrm{H}), 5.47(\mathrm{~s}, 1 \mathrm{H}), 4.28(\mathrm{dd}, J=11.0,4.6 \mathrm{~Hz}, 1 \mathrm{H}), 4.03-3.94(\mathrm{~m}, 2 \mathrm{H}), 3.89-3.76(\mathrm{~m}$, $1 \mathrm{H}), 3.79(\mathrm{~s}, 3 \mathrm{H}), 3.67(\mathrm{dd}, J=10.2,5.6 \mathrm{~Hz}, 1 \mathrm{H}), 1.86(\mathrm{dq}, J=12.9,5.1 \mathrm{~Hz}, 1 \mathrm{H}), 1.63$ (d, $J=$ $12.9 \mathrm{~Hz}, 1 \mathrm{H}), 1.06(\mathrm{~s}, 9 \mathrm{H}) ;{ }^{13} \mathrm{C}$ NMR $\left(100 \mathrm{MHz}, \mathrm{CDCl}_{3}\right) \delta 159.8,135.6,134.7,133.4,131.2$, 129.6, 127.6, 127.6, 127.3, 113.4, 100.9, 66.8, 66.7, 55.2, 28.0, 26.7, 26.5, 19.2; FT-IR (neat) $v 2856,1615,1517,1428,1249,1111,824,741,702,607 \mathrm{~cm}^{-1}$; MS (ESI) $\mathrm{m} / z: 367,485$ (100) $\left[(\mathrm{M}+\mathrm{Na})^{+}\right]$; HRMS (ESI) calcd for $\mathrm{C}_{28} \mathrm{H}_{34} \mathrm{NaO}_{4} \mathrm{Si}\left[(\mathrm{M}+\mathrm{Na})^{+}\right]$485.2124, found 485.2100.

OPMB
OPO-3-(4-Methoxybenzyloxy)-4-(tert-butyldiphenylsilyloxy)butan-1-ol added diisobutylaluminium hydride $(1.02 \mathrm{M}$ in hexane, $246 \mathrm{~mL}, 251 \mathrm{mmol})$ at $-78^{\circ} \mathrm{C}$ over 3.5 $\mathrm{h}$ and the mixture was stirred at $-40{ }^{\circ} \mathrm{C}$ for $7 \mathrm{~h}$. The reaction was quenched with saturated Rochelle salt ( $85 \mathrm{~g}, 302 \mathrm{mmol}, 200 \mathrm{~mL}$ ). After stirring at room temperature for $14 \mathrm{~h}$, the mixture was extracted with EtOAc $(200 \mathrm{~mL})$. The extracts were washed with brine $(300 \mathrm{~mL})$, dried, and concentrated. The residue was purified by column chromatography $\left(\mathrm{SiO}_{2} 1400 \mathrm{~g}\right.$, hexane/EtOAc $=8: 1$ to $3: 1)$ to give $\mathbf{S 2}(28.9 \mathrm{~g}, 62.2 \mathrm{mmol}, 74 \%)$ as a colorless oil. $[\alpha]_{\mathrm{D}}{ }^{21}-31.6$ (c 1.00, $\left.\mathrm{CHCl}_{3}\right) ;{ }^{1} \mathrm{H}$ NMR (400 MHz, $\left.\mathrm{CDCl}_{3}\right) \delta 7.68(\mathrm{~d}, J=6.3 \mathrm{~Hz}, 4 \mathrm{H}), 7.44-7.36(\mathrm{~m}, 6 \mathrm{H})$, $7.20(\mathrm{~d}, J=8.6 \mathrm{~Hz}, 2 \mathrm{H}), 6.85(\mathrm{~d}, J=8.6 \mathrm{~Hz}, 2 \mathrm{H}), 4.60(\mathrm{~d}, J=11.2 \mathrm{~Hz}, 1 \mathrm{H}), 4.41(\mathrm{~d}, J=11.2$ $\mathrm{Hz}, 1 \mathrm{H}), 3.80$ (s, 3H), 3.78-3.65 (m, 5H), 2.35 (br, 1H), 1.85-1.78 (m, 2H), $1.06(\mathrm{~s}, 9 \mathrm{H}) ;{ }^{13} \mathrm{C}$ NMR $\left(100 \mathrm{MHz}, \mathrm{CDCl}_{3}\right) \delta 159.2,135.6,135.5,133.2,133.2,130.4,129.7,129.4,127.7,113.8$, 78.4, 71.7, 65.8, 60.4, 55.2, 34.1, 26.7, 19.1; FT-IR (neat) v 3430, 2931, 1613, 1513, 1428, 1248, 1111, 822, 741, $702 \mathrm{~cm}^{-1}$; MS (ESI) $m / z: 121,487$ (100) [(M+Na) ${ }^{+}$; HRMS (ESI) calcd for $\mathrm{C}_{28} \mathrm{H}_{36} \mathrm{NaO}_{4} \mathrm{Si}\left[(\mathrm{M}+\mathrm{Na})^{+}\right]$487.2280, found 487.2281.

Spectroscopic data in accord with the literature data. ${ }^{1}$

OPMB Ethyl (S,E)-5-(4-methoxybenzyloxy)-6-(tert-butyldiphenylsilyl$\mathrm{EtO}_{2} \mathrm{C} \sim$ OTBDPS oxy)hex-2-enoate (14): To a solution of oxalyl chloride $(369 \mu \mathrm{L}, 4.30$ $\mathrm{mmol})$ in $\mathrm{CH}_{2} \mathrm{Cl}_{2}(13 \mathrm{~mL})$ was added dropwise a solution of DMSO $(611 \mu \mathrm{L}, 9.00 \mathrm{mmol})$ in 
$\mathrm{CH}_{2} \mathrm{Cl}_{2}(4 \mathrm{~mL})$ at $-78{ }^{\circ} \mathrm{C}$, and the mixture was stirred at $-78^{\circ} \mathrm{C}$ for $30 \mathrm{~min}$. To this mixture was added a solution of $\mathbf{S 2}(1.0 \mathrm{~g}, 2.15 \mathrm{mmol})$ in $\mathrm{CH}_{2} \mathrm{Cl}_{2}(5 \mathrm{~mL})$, and it was stirred at $-78^{\circ} \mathrm{C}$ for an additional $1 \mathrm{~h}$. Then triethylamine $(2.4 \mathrm{~mL}, 17.2 \mathrm{mmol})$ was added. After stirring at $-40{ }^{\circ} \mathrm{C}$ for $20 \mathrm{~min}$, a solution of (1-(ethoxycarbonyl)ethylidene)triphenylphosphorane (1.9 g, $5.38 \mathrm{mmol})$ in $\mathrm{CH}_{2} \mathrm{Cl}_{2}(6 \mathrm{~mL})$ was added and stirring was continued at $0{ }^{\circ} \mathrm{C}$ for $14 \mathrm{~h}$. The mixture was diluted with water $(20 \mathrm{~mL})$ and extracted with EtOAc $(100 \mathrm{~mL})$. The extracts were washed with water $(20 \mathrm{~mL})$, brine $(20 \mathrm{~mL})$, dried, and concentrated. The residue was purified by column chromatography $\left(\mathrm{SiO}_{2} 160 \mathrm{~g}\right.$, hexane/EtOAc $=20: 1$ to 4:1) to give $14(1.0 \mathrm{~g}, 1.88$ mmol, $90 \%)$ as a pale yellow oil. $[\alpha]_{\mathrm{D}}{ }^{21}-17.6\left(c 1.00, \mathrm{CHCl}_{3}\right) ;{ }^{1} \mathrm{H}$ NMR $\left(400 \mathrm{MHz}, \mathrm{CDCl}_{3}\right) \delta$ $7.66(\mathrm{~d}, J=7.6 \mathrm{~Hz}, 4 \mathrm{H}), 7.42-7.37$ (m, 6H), $7.19(\mathrm{~d}, J=8.4 \mathrm{~Hz}, 2 \mathrm{H}), 6.95$ (dt, $J=15.6,7.3 \mathrm{~Hz}$, $1 \mathrm{H}), 6.83(\mathrm{~d}, J=8.4 \mathrm{~Hz}, 2 \mathrm{H}), 5.85(\mathrm{~d}, J=15.6 \mathrm{~Hz}, 1 \mathrm{H}), 4.50(\mathrm{~d}, J=11.3 \mathrm{~Hz}, 1 \mathrm{H}), 4.41(\mathrm{~d}, J$ $=11.3 \mathrm{~Hz}, 1 \mathrm{H}), 4.19(\mathrm{q}, J=7.1 \mathrm{~Hz}, 2 \mathrm{H}), 3.79(\mathrm{~s}, 3 \mathrm{H}), 3.72(\mathrm{dd}, J=9.9,4.6 \mathrm{~Hz}, 1 \mathrm{H}), 3.62(\mathrm{dd}$, $J=9.9,5.6 \mathrm{~Hz}, 1 \mathrm{H}), 3.60-3.56(\mathrm{~m}, 1 \mathrm{H}), 2.55(\mathrm{dd}, J=14.2,5.6 \mathrm{~Hz}, 1 \mathrm{H}), 2.43$ (dd, $J=14.2,7.2$ $\mathrm{Hz}, 1 \mathrm{H}), 1.30(\mathrm{t}, J=7.1 \mathrm{~Hz}, 3 \mathrm{H}) 1.05(\mathrm{~s}, 9 \mathrm{H}) ;{ }^{13} \mathrm{C} \mathrm{NMR}\left(100 \mathrm{MHz}, \mathrm{CDCl}_{3}\right) \delta 166.3,159.1$, $145.3,135.5,135.5,133.3,133.2,130.3,129.6,129.2,127.6,123.3,113.3,77.9,71.6,65.2$, $60.3,60.0,55.1,34.6,26.7,20.9,19.1,14.2,14.1$; FT-IR (neat) v 2931, 1718, 1512, 1428, 1247 , 1172, 1111, 823, 702, $504 \mathrm{~cm}^{-1}$; MS (EI) $\mathrm{m} / \mathrm{z}:$ 57, 69, 121 (100), 163, 199, 241, 309, 475, 532 $\left(\mathrm{M}^{+}\right)$; HRMS (EI) calcd for $\mathrm{C}_{32} \mathrm{H}_{40} \mathrm{O}_{5} \mathrm{Si}\left(\mathrm{M}^{+}\right)$532.2629, found 532.2663.

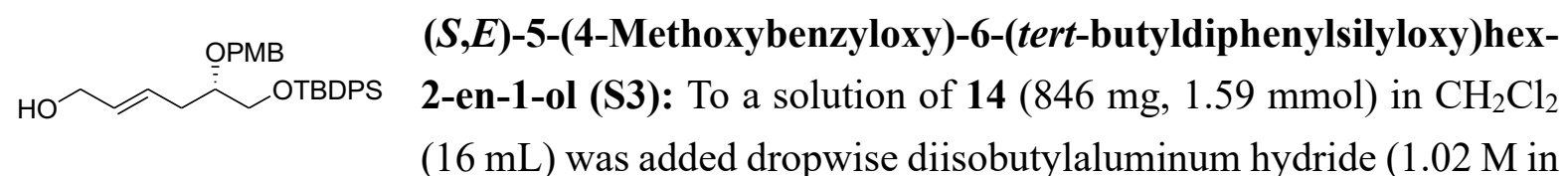
hexane, $4.7 \mathrm{~mL}, 4.67 \mathrm{mmol}$ ) at $-78{ }^{\circ} \mathrm{C}$ and the mixture was stirred at $-40{ }^{\circ} \mathrm{C}$ for $13 \mathrm{~h}$. The reaction was quenched with saturated Rochelle salt $(10 \mathrm{~mL})$. After being stirred at room temperature for $9 \mathrm{~h}$, the mixture was extracted with EtOAc $(40 \mathrm{~mL})$. The extracts were washed with brine $(10 \mathrm{~mL})$, dried, and concentrated. The residue was purified by column chromatography $\left(\mathrm{SiO}_{2} 32 \mathrm{~g}\right.$, hexane/EtOAc $\left.=4: 1\right)$ to give $\mathbf{S 3}(666 \mathrm{mg}, 1.36 \mathrm{mmol}, 94 \%)$ as a colorless oil. $[\alpha]_{\mathrm{D}}{ }^{21}-16.7\left(c 1.00, \mathrm{CHCl}_{3}\right) ;{ }^{1} \mathrm{H}$ NMR (400 MHz, $\left.\mathrm{CDCl}_{3}\right) \delta 7.67(\mathrm{~d}, J=6.8 \mathrm{~Hz}$, $4 \mathrm{H}), 7.42-7.37(\mathrm{~m}, 6 \mathrm{H}), 7.19(\mathrm{~d}, J=8.4 \mathrm{~Hz}, 2 \mathrm{H}), 6.84(\mathrm{~d}, J=8.4 \mathrm{~Hz}, 2 \mathrm{H}), 5.61-5.59(\mathrm{~m}, 2 \mathrm{H})$, $4.55(\mathrm{~d}, J=11.4 \mathrm{~Hz}, 1 \mathrm{H}), 4.44$ (d, $J=11.4 \mathrm{~Hz}, 1 \mathrm{H}), 4.03$ (br, 2H), 3.79 (s, 3H), 3.71 (dd, $J=$ 10.6, 5.6 Hz, 1H), 3.63 (dd, $J=10.6,5.2 \mathrm{~Hz}, 1 \mathrm{H}), 3.55-3.49$ (m, 1H), 2.39-2.25 (m, 2H), 1.15 (br, 1H) $1.06(\mathrm{~s}, 9 \mathrm{H}) ;{ }^{13} \mathrm{C} \mathrm{NMR}\left(100 \mathrm{MHz}, \mathrm{CDCl}_{3}\right) \delta 159.0,135.6,135.6,133.4,133.4,131.4$, $130.8,129.6,129.3,128.8,127.6,113.6,78.9$, 71.5, 65.4, 63.6, 60.3, 55.2, 34.3, 26.8, 19.1, 14.4; FT-IR (neat) v 3406, 2930, 1613, 1513, 1428, 1247, 1111, 822, 741, $702 \mathrm{~cm}^{-1}$; MS (ESI) $m / z: 279,513(100)\left[(\mathrm{M}+\mathrm{Na})^{+}\right]$; HRMS (ESI) calcd for $\mathrm{C}_{30} \mathrm{H}_{38} \mathrm{NaO}_{4} \mathrm{Si}\left[(\mathrm{M}+\mathrm{Na})^{+}\right]$513.2437, found 513.2406. 
每 $\stackrel{\text { OPMB }}{\text { OTBDPS }}$

((2S,3S)-3-((S)-2-(4-Methoxybenzyloxy)-3-(tert-butyldiphenylsilyloxy)propyl)-oxiran-2-yl)methanol (15): ${ }^{2}$ To a solution of dried MS-4Å powder $(200 \mathrm{mg})$ in $\mathrm{CH}_{2} \mathrm{Cl}_{2}(200 \mathrm{~mL})$ were added titanium tetraisopropoxide $(49.4 \mu \mathrm{L}$, $0.16 \mathrm{mmol})$ and diethyl $\mathrm{L}-(+)$-tartrate $(37 \mathrm{mg}, 0.18 \mathrm{mmol})$ in $\mathrm{CH}_{2} \mathrm{Cl}_{2}(2 \mathrm{~mL})$ at $-30{ }^{\circ} \mathrm{C}$ and the mixture was stirred at $-30{ }^{\circ} \mathrm{C}$ for $30 \mathrm{~min}$. To this mixture was added tert-butyl hydroperoxide (4.04 $\mathrm{M}$ in toluene, $606 \mu \mathrm{L}, 2.45 \mathrm{mmol}$ ), and it was stirred at $-30^{\circ} \mathrm{C}$ for an additional $30 \mathrm{~min}$. Then a solution of $\mathbf{S 3}$ (400 $\mathrm{mg}, 0.82 \mathrm{mmol})$ in $\mathrm{CH}_{2} \mathrm{Cl}_{2}(4 \mathrm{~mL})$ was added and stirring was continued at $-30{ }^{\circ} \mathrm{C}$ for $22 \mathrm{~h}$. After stirring at $-25^{\circ} \mathrm{C}$ for $18 \mathrm{~h}$, the reaction was quenched with $50 \%$ sodium thiosulfate solution $(14 \mathrm{~mL})$. The mixture was filtered through Celite and extracted with EtOAc $(30 \mathrm{~mL})$. The extracts were washed with brine $(10 \mathrm{~mL})$, dried, and concentrated. The residue was purified by column chromatography $\left(\mathrm{SiO}_{2} 22 \mathrm{~g}\right.$, hexane/EtOAc $=3: 1$ to $2: 1)$ to give $15(381 \mathrm{mg}, 0.75 \mathrm{mmol}, 92 \%)$ as a colorless oil. [ $\alpha]_{\mathrm{D}}{ }^{21}-24.7$ (c 1.00, $\left.\mathrm{CHCl}_{3}\right) ;{ }^{1} \mathrm{H}$ NMR $\left(400 \mathrm{MHz}, \mathrm{CDCl}_{3}\right) \delta 7.66(\mathrm{~d}, J=6.6 \mathrm{~Hz}, 4 \mathrm{H}), 7.45-7.35(\mathrm{~m}, 6 \mathrm{H}), 7.21(\mathrm{~d}, J$ $=8.5 \mathrm{~Hz}, 2 \mathrm{H}), 6.85(\mathrm{~d}, J=8.5 \mathrm{~Hz}, 2 \mathrm{H}), 4.60(\mathrm{~d}, J=11.1 \mathrm{~Hz}, 1 \mathrm{H}), 4.45(\mathrm{~d}, J=11.1 \mathrm{~Hz}, 1 \mathrm{H})$, $3.86(\mathrm{dd}, J=5.6,3.0 \mathrm{~Hz}, 1 \mathrm{H}), 3.83(\mathrm{dd}, J=5.6,3.0 \mathrm{~Hz}, 1 \mathrm{H}), 3.79(\mathrm{~s}, 3 \mathrm{H}), 3.75-3.63(\mathrm{~m}, 3 \mathrm{H})$, 3.59-3.53 (m, 1H), 3.07 (br, 1H), $2.92(\mathrm{br}, 1 \mathrm{H}), 1.85-1.75(\mathrm{~m}, 2 \mathrm{H}), 1.05(\mathrm{~s}, 9 \mathrm{H})$; ${ }^{13} \mathrm{C}$ NMR (100 $\left.\mathrm{MHz}_{\mathrm{CDCl}}\right) \delta 171.0,159.0,135.4,133.2,133.1,130.5,129.5,129.1,127.5,113.6,71.8,65.7$, 61.6, 60.2, 58.9, 55.0, 53.2, 34.2, 26.6, 20.8, 19.0, 14.0; FT-IR (neat) v 3450, 2929, 1746, 1612 , 1512, 1427, 1110, 702, $503 \mathrm{~cm}^{-1}$; MS (ESI) $\mathrm{m} / \mathrm{z}: 529(100)\left[(\mathrm{M}+\mathrm{Na})^{+}\right]$; HRMS (ESI) calcd for $\mathrm{C}_{30} \mathrm{H}_{38} \mathrm{NaO}_{5} \mathrm{Si}\left[(\mathrm{M}+\mathrm{Na})^{+}\right]$529.2386, found 529.2359.

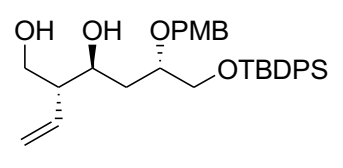

(2R,3S,5S)-3-Hydroxy-5-(4-methoxybenzyloxy)-6-(tert-butyldiphenylsilyloxy)-2-vinyl-hexan-1-ol (S4): To a solution of copper (I) iodide (4.49 $\mathrm{g}, 23.6 \mathrm{mmol})$ in THF $(150 \mathrm{~mL})$ was added dropwise vinylmagnesium bromide (1.0 $\mathrm{M}$ in THF, $117.8 \mathrm{~mL}, 117.8 \mathrm{mmol})$ at $-26{ }^{\circ} \mathrm{C}$. After stirring at $26^{\circ} \mathrm{C}$ for $3 \mathrm{~h}$, a solution of $15(5.97 \mathrm{~g}, 11.8 \mathrm{mmol})$ in THF $(240 \mathrm{~mL})$ was added, and the mixture was stirred at $-26{ }^{\circ} \mathrm{C}$ for $19 \mathrm{~h}$. The reaction was quenched with saturated $\mathrm{NH}_{4} \mathrm{Cl}(400 \mathrm{~mL})$. The mixture was filtered through Celite and extracted with EtOAc $(400 \mathrm{~mL})$. The extracts were washed with brine $(400 \mathrm{~mL})$, dried, and concentrated. To a solution of the resulting mixture in THF $(400 \mathrm{~mL})$ was added $\mathrm{NaIO}_{4}(2.10 \mathrm{~g}, 9.82 \mathrm{mmol})$, and the mixture was stirred at room temperature for $15 \mathrm{~h}$. The reaction was quenched with brine $(400 \mathrm{~mL})$ and the mixture was extracted with EtOAc $(400 \mathrm{~mL})$. The extracts were dried, and concentrated. The residue was purified by flash chromatography $\left(\mathrm{SiO}_{2} 250 \mathrm{~g}\right.$, hexane/EtOAc $\left.=4: 1\right)$ to afford $\mathbf{S 4}(5.48 \mathrm{~g}, 10.2$ mmol, 87\%) as a colorless oil. $[\alpha]_{\mathrm{D}}{ }^{24}-32.5$ (c 0.90, $\left.\mathrm{CHCl}_{3}\right) ;{ }^{1} \mathrm{H} \mathrm{NMR}\left(400 \mathrm{MHz}, \mathrm{CDCl}_{3}\right) \delta$ 7.67-7.65 (m, 4H), 7.46-7.37 (m, 6H), $7.18(\mathrm{~d}, J=8.2 \mathrm{~Hz}, 2 \mathrm{H}), 6.86(\mathrm{~d}, J=8.2 \mathrm{~Hz}, 2 \mathrm{H}), 5.54$ $(\mathrm{dt}, J=18.4,9.2 \mathrm{~Hz}, 1 \mathrm{H}), 5.15-5.11(\mathrm{~m}, 2 \mathrm{H}), 4.56(\mathrm{~d}, J=11.2 \mathrm{~Hz}, 1 \mathrm{H}), 4.41(\mathrm{~d}, J=11.2 \mathrm{~Hz}$, $1 \mathrm{H}), 3.91(\mathrm{t}, J=8.8 \mathrm{~Hz}, 1 \mathrm{H}), 3.80(\mathrm{~s}, 3 \mathrm{H}), 3.78(\mathrm{~d}, J=4.8 \mathrm{~Hz}, 2 \mathrm{H}), 3.67-3.62(\mathrm{~m}, 3 \mathrm{H}), 2.32-$ $2.25(\mathrm{~m}, 1 \mathrm{H}), 1.87$ (dd, $J=14.8,4.8 \mathrm{~Hz}, 1 \mathrm{H}), 1.72(\mathrm{ddd}, J=14.8,8.8,3.2 \mathrm{~Hz}, 1 \mathrm{H}), 1.54$ (br, 
2H), 1.05 (s, 9H); ${ }^{13} \mathrm{C}$ NMR (100 MHz, $\left.\mathrm{CDCl}_{3}\right) \delta 136.1,135.6,133.2,133.1,129.9,129.8$, $129.6,127.7,118.0,113.9,77.1,72.4,71.9,65.9,65.3,55.3,51.8,36.0,26.8,19.1$; FT-IR (neat) $v$ 3411, 2930, 2862, 1611, 1512, 1464, 1248, 1107, 819, 703, $499 \mathrm{~cm}^{-1}$; MS (EI) m/z: 69, 121 (100), 199, 333, $534\left(\mathrm{M}^{+}\right)$; HRMS (EI) calcd for $\mathrm{C}_{32} \mathrm{H}_{42} \mathrm{O}_{5} \mathrm{Si}\left(\mathrm{M}^{+}\right)$534.2802, found 534.2802.

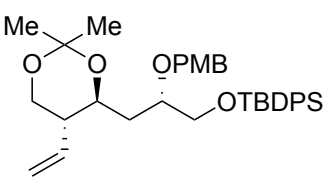

(4S,5R)-4-((S)-2-(4-Methoxybenzyloxy)-3-(tert-butyldiphenylsilyloxy)propyl)-2,2-dimethyl-5-vinyl-1,3-dioxane (16): To an ice-cooled solution of $\mathbf{S 4}(1.49 \mathrm{~g}, 2.78 \mathrm{mmol})$ in $\mathrm{CH}_{2} \mathrm{Cl}_{2}(19 \mathrm{~mL})$ were added 2,2dimethoxypropane $(3.42 \mathrm{~mL}, 27.8 \mathrm{mmol})$ and PPTS $(70.0 \mathrm{mg}, 278$ $\mu \mathrm{mol})$, and the mixture was stirred at room temperature for $5.5 \mathrm{~h}$. The reaction was quenched with $\mathrm{NaHCO}_{3}(230 \mathrm{mg}, 2.78 \mathrm{mmol})$ and the mixture was concentrated. The residue was purified by flash chromatography $\left(\mathrm{SiO}_{2} 80 \mathrm{~g}\right.$, hexane/EtOAc $\left.=10: 1\right)$ to give $16(1.44 \mathrm{~g}, 2.51$ mmol, 90\%) as a colorless oil. $[\alpha]_{\mathrm{D}}{ }^{21}-31.8\left(c 1.00, \mathrm{CHCl}_{3}\right) ;{ }^{1} \mathrm{H} \mathrm{NMR}\left(400 \mathrm{MHz}, \mathrm{CDCl}_{3}\right) \delta 7.68$ $(\mathrm{t}, J=6.5 \mathrm{~Hz}, 4 \mathrm{H}), 7.41-7.34(\mathrm{~m}, 6 \mathrm{H}), 7.22(\mathrm{~d}, J=8.4 \mathrm{~Hz}, 2 \mathrm{H}), 6.85(\mathrm{~d}, J=8.5 \mathrm{~Hz}, 2 \mathrm{H}), 5.45$ $(\mathrm{dt}, J=17.2,10.1 \mathrm{~Hz}, 1 \mathrm{H}), 5.10(\mathrm{~d}, J=17.2 \mathrm{~Hz}, 1 \mathrm{H}), 5.08(\mathrm{~d}, J=10.1 \mathrm{~Hz}, 1 \mathrm{H}), 4.66(\mathrm{~d}, J=$ $11.1 \mathrm{~Hz}, 1 \mathrm{H}), 4.40$ (d, $J=11.1 \mathrm{~Hz}, 1 \mathrm{H}), 3.89$ (t, $J=10.0 \mathrm{~Hz}, 1 \mathrm{H}), 3.79$ (s, 3H), 3.72 (br, 1H), 3.69-3.65 (m, 4H), 2.24-2.15 (m, 1H), $1.78(\mathrm{t}, J=12.4 \mathrm{~Hz}, 1 \mathrm{H}), 1.41(\mathrm{t}, J=12.4 \mathrm{~Hz}, 1 \mathrm{H}), 1.38$ $(\mathrm{s}, 6 \mathrm{H}), 1.06(\mathrm{~s}, 9 \mathrm{H}) ;{ }^{13} \mathrm{C} \mathrm{NMR}\left(100 \mathrm{MHz}, \mathrm{CDCl}_{3}\right) \delta 159.0,135.6,135.6,134.6,133.5,131.2$, 129.5, 127.6, 118.6, 113.6, 98.0, 75.3, 72.4, 68.5, 67.0, 64.0, 60.3, 55.2, 45.8, 36.4, 29.7, 26.8, 21.0, 19.1, 19.1, 14.1; FT-IR (neat) v 2931, 1613, 1513, 1427, 1379, 1246, 1111, 822, 702, 505 $\mathrm{cm}^{-1}$; MS (ESI) m/z: $597(100)\left[(\mathrm{M}+\mathrm{Na})^{+}\right]$; HRMS (ESI) calcd for $\mathrm{C}_{35} \mathrm{H}_{46} \mathrm{NaO}_{5} \mathrm{Si}\left[(\mathrm{M}+\mathrm{Na})^{+}\right]$ 597.3012, found 597.3017.

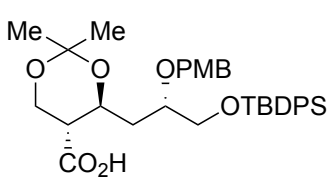

(4S,5R)-4-((S)-2-(4-Methoxybenzyloxy)-3-(tert-butyldiphenylsilyloxy)propyl)-2,2-dimethyl-1,3-dioxane-5-carboxylic acid (9) : To a solution of $16(300 \mathrm{mg}, 0.51 \mathrm{mmol})$ in 1,4-dioxane $(3.84 \mathrm{~mL})$ and water (1.26 mL) were added 2,6-lutidine $(119 \mu \mathrm{L}, 1.02 \mathrm{mmol})$, osmium tetroxide $\left(0.16 \mathrm{M}\right.$ in $\left.\mathrm{H}_{2} \mathrm{O}, 65 \mu \mathrm{L}, 10.2 \mu \mathrm{mol}\right)$, and sodium periodate (439 $\left.\mathrm{mg}, 2.05 \mathrm{mmol}\right)$, and the mixture was stirred at room temperature for $13 \mathrm{~h}$. To this mixture was added osmium tetroxide $\left(0.16 \mathrm{M}\right.$ in $\left.\mathrm{H}_{2} \mathrm{O}, 65 \mu \mathrm{L}, 10.2 \mu \mathrm{mol}\right)$ again, and it was stirred at room temperature for an additional $5 \mathrm{~h}$. The mixture was diluted with water $(5 \mathrm{~mL})$ and $\mathrm{CH}_{2} \mathrm{Cl}_{2}(10 \mathrm{~mL})$, and extracted with $\mathrm{CH}_{2} \mathrm{Cl}_{2}(45 \mathrm{~mL})$. The extracts were washed with brine $(5 \mathrm{~mL})$, dried, and concentrated to give crude aldehyde.

To an ice-cooled solution of the resulting aldehyde in $t$-butyl alcohol $(3.72 \mathrm{~mL})$ and water $(0.93$ $\mathrm{mL})$ were added 2-methyl-2-butene $(1.1 \mathrm{~mL}, 10.2 \mathrm{mmol})$, sodium dihydrogen phosphate dihydrate $(240 \mathrm{mg}, 1.54 \mathrm{mmol})$, and sodium chlorite $(185 \mathrm{mg}, 2.05 \mathrm{mmol})$, and the mixture was stirred at room temperature for $5 \mathrm{~h}$. The reaction was quenched with saturated $\mathrm{NH}_{4} \mathrm{Cl}(10$ $\mathrm{mL})$ and the mixture was extracted with EtOAc $(45 \mathrm{~mL})$. The extracts were dried and 
concentrated. The residue was purified by flash chromatography $\left(\mathrm{SiO}_{2} 20 \mathrm{~g}\right.$, hexane/EtOAc $=$ $3: 1)$ to afford $9(272 \mathrm{mg}, 0.46 \mathrm{mmol}, 90 \%, 2$ steps $)$ as a colorless oil. [ $\alpha]_{\mathrm{D}}{ }^{23}-37.6(c) 1.33$, $\left.\mathrm{CHCl}_{3}\right) ;{ }^{1} \mathrm{H}$ NMR $\left(400 \mathrm{MHz}, \mathrm{CDCl}_{3}\right) \delta$ 7.67-7.65 (m, 4H), 7.43-7.33 (m, 6H), $7.21(\mathrm{~d}, J=8.6$ $\mathrm{Hz}, 2 \mathrm{H}), 6.83(\mathrm{~d}, J=8.6 \mathrm{~Hz}, 2 \mathrm{H}), 4.65(\mathrm{~d}, J=11.1 \mathrm{~Hz}, 1 \mathrm{H}), 4.41(\mathrm{~d}, J=11.1 \mathrm{~Hz}, 1 \mathrm{H}), 4.22$ (t, $J=9.2 \mathrm{~Hz}, 1 \mathrm{H}), 4.00$ (d, $J=11.3 \mathrm{~Hz}, 1 \mathrm{H}), 3.92(\mathrm{dd}, J=11.3,5.3 \mathrm{~Hz}, 1 \mathrm{H}), 3.78$ (s, 3H), 3.77 (br, $1 \mathrm{H}), 3.71-3.61(\mathrm{~m}, 2 \mathrm{H}), 2.58-2.50(\mathrm{~m}, 1 \mathrm{H}), 1.81(\mathrm{t}, J=12.0 \mathrm{~Hz}, 1 \mathrm{H}), 1.59$ (t, $J=12.0 \mathrm{~Hz}$, $1 \mathrm{H}), 1.35(\mathrm{~s}, 6 \mathrm{H}), 1.04(\mathrm{~s}, 9 \mathrm{H}) ;{ }^{13} \mathrm{C} \mathrm{NMR}\left(100 \mathrm{MHz}, \mathrm{CDCl}_{3}\right) \delta 159.2,135.7,135.6,133.5$, 130.5, 129.6, 127.7, 133.7, 98.5, 75.2, 72.3, 66.7, 66.4, 61.2, 55.3, 47.0, 37.3, 29.2, 26.9, 19.3, 19.2; FT-IR (neat) v 2932, 1732, 1611, 1513, 1428, 1249, 1112, 822, 702, $507 \mathrm{~cm}^{-1}$; MS (ESI) $m / z: 615(100)\left[(\mathrm{M}+\mathrm{Na})^{+}\right], 1209$; HRMS (ESI) calcd for $\mathrm{C}_{34} \mathrm{H}_{44} \mathrm{NaO}_{7} \mathrm{Si}\left[(\mathrm{M}+\mathrm{Na})^{+}\right] 615.2754$, found 615.2785 .

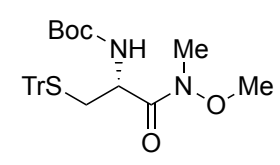

(R)- $N$-Methyl- $N$-methoxy-2-tert-butoxycarbonylamino-3-(triphenylmethylthio)propionamide (S5): To a solution of $N$-(tert-butoxycarbonyl)$(S)$-trityl-L-cysteine $(1.0 \mathrm{~g}, 2.16 \mathrm{mmol})$ in $\mathrm{CH}_{2} \mathrm{Cl}_{2}(11 \mathrm{~mL})$ were added $N$ methoxymethylamine (274 mg, $2.81 \mathrm{mmol}), N, N$-diisopropylethylamine ( $924 \mu \mathrm{L}, 5.40 \mathrm{mmol})$, 1-hydroxybenzotriazole (380 $\mathrm{mg}, 2.81 \mathrm{mmol})$, and 1-(3-dimethylaminopropyl)-3ethylcarbodiimide hydrochloride $(539 \mathrm{mg}, 2.81 \mathrm{mmol})$, and the mixture was stirred at room temperature for $3 \mathrm{~h}$. The reaction was quenched with $1 \mathrm{M} \mathrm{HCl}(5.4 \mathrm{~mL})$ at $0{ }^{\circ} \mathrm{C}$ and the mixture was extracted with EtOAc $(10 \mathrm{~mL})$. The extracts were washed with saturated $\mathrm{NaHCO}_{3}(15 \mathrm{~mL})$ and brine $(15 \mathrm{~mL})$, dried, and concentrated. The residue was purified by column chromatography $\left(\mathrm{SiO}_{2} 40 \mathrm{~g}\right.$, hexane/EtOAc $=4: 1$ to 3:1) to afford $\mathbf{S 5}(1.09 \mathrm{~g}, 2.15 \mathrm{mmol}$, $100 \%)$ as a white amorphous. $[\alpha]_{\mathrm{D}}{ }^{28}-11.9\left(c 1.00, \mathrm{CHCl}_{3}\right) ;{ }^{1} \mathrm{H} \mathrm{NMR}\left(400 \mathrm{MHz}, \mathrm{CDCl}_{3}\right) \delta 7.40$ $(\mathrm{d}, J=7.3 \mathrm{~Hz}, 6 \mathrm{H}), 7.29-7.18(\mathrm{~m}, 9 \mathrm{H}), 5.11(\mathrm{~d}, J=7.8 \mathrm{~Hz}, 1 \mathrm{H}), 4.75$ (br, 1H), $3.64(\mathrm{~s}, 3 \mathrm{H})$, 3.14 (s, 3H), $2.54(\mathrm{dd}, J=12.0,4.4 \mathrm{~Hz}, 1 \mathrm{H}), 2.37(\mathrm{dd}, J=12.0,7.8 \mathrm{~Hz}, 1 \mathrm{H}), 1.43(\mathrm{~s}, 9 \mathrm{H}) ;{ }^{13} \mathrm{C}$ NMR $\left(125 \mathrm{MHz}, \mathrm{CDCl}_{3}\right) \delta 170.7,154.9,144.2,129.3,127.6,126.5,79.3,66.4,61.2,60.0$, 49.5, 33.8, 31.8, 28.1, 20.7, 15.0, 13.9; FT-IR (neat) v 3426, 2979, 1724, 1185, 1048, 911, 857, $769,623,503 \mathrm{~cm}^{-1}$; MS (ESI) $\mathrm{m} / z: 243,529(100)\left[(\mathrm{M}+\mathrm{Na})^{+}\right], 1036$; HRMS (ESI) calcd for $\mathrm{C}_{29} \mathrm{H}_{34} \mathrm{NaO}_{4} \mathrm{~S}\left[(\mathrm{M}+\mathrm{Na})^{+}\right]$529.2137, found 529.2094.

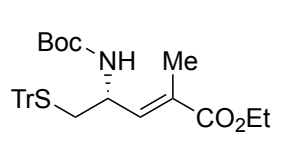

Ethyl-(E)-(R)-4-tert-butoxycarbonylamino-2-methyl-5-(triphenylmethylthio)-pentenoate (S6): To a solution of S5 (1.09 g, $2.15 \mathrm{mmol})$ in toluene $(21 \mathrm{~mL})$ was added diisobutylaluminum hydride $(1.04 \mathrm{M}$ in hexane, $4.6 \mathrm{~mL}$, $4.73 \mathrm{mmol}$ ) at $-78{ }^{\circ} \mathrm{C}$, and the mixture was stirred at $-78{ }^{\circ} \mathrm{C}$ for $1 \mathrm{~h}$. The mixture was added to saturated Rochelle salt $(50 \mathrm{~mL})$ via cannula. After stirring at $0{ }^{\circ} \mathrm{C}$ for $1 \mathrm{~h}$, the mixture was extracted with $\mathrm{Et}_{2} \mathrm{O}(50 \mathrm{~mL})$. The extracts were washed with brine $(50 \mathrm{~mL})$, dried, and concentrated to give crude aldehyde.

To an ice-cooled solution of the resulting aldehyde in toluene $(15 \mathrm{~mL})$ was added (1- 
(ethoxycarbonyl)ethylidene)triphenylphosphorane (1.44 g, $3.96 \mathrm{mmol})$. After being stirred at room temperature for $5 \mathrm{~h}$, the mixture was concentrated. The residue was purified by column chromatography $\left(\mathrm{SiO}_{2} 120 \mathrm{~g}\right.$, hexane/EtOAc $\left.=7: 1\right)$ to afford $\mathbf{S 6}(1.00 \mathrm{~g}, 1.89 \mathrm{mmol}, 88 \%)$ as a white amorphous. $[\alpha]_{\mathrm{D}}{ }^{23}+18.7\left(c 1.04, \mathrm{CHCl}_{3}\right) ;{ }^{1} \mathrm{H} \mathrm{NMR}\left(400 \mathrm{MHz}, \mathrm{CDCl}_{3}\right) \delta 7.39(\mathrm{~d}, J=$ 7.6 Hz, 6H), 7.30-7.20 (m, 9H), $6.32(\mathrm{~d}, J=9.2 \mathrm{~Hz}, 1 \mathrm{H}), 4.54-4.42(\mathrm{~m}, 1 \mathrm{H}), 4.37-4.23(\mathrm{~m}, 1 \mathrm{H})$, $4.10(\mathrm{q}, J=7.2 \mathrm{~Hz}, 2 \mathrm{H}), 2.35$ (dd, $J=12.4,7.6 \mathrm{~Hz}, 1 \mathrm{H}), 2.27$ (dd, $J=12.4,6.4 \mathrm{~Hz}, 1 \mathrm{H}), 1.69$ (s, 3H), $1.34(\mathrm{~s}, 9 \mathrm{H}), 1.20(\mathrm{t}, J=7.2 \mathrm{~Hz}, 3 \mathrm{H}) ;{ }^{13} \mathrm{C} \mathrm{NMR}\left(100 \mathrm{MHz}, \mathrm{CDCl}_{3}\right) \delta 167.4,154.6$, 144.4, 144.3, 139.8, 129.4, 127.8, 126.6, 126.5, 79.3, 66.8, 60.5, 48.7, 28.2, 14.0, 12.6; FT-IR (neat) $v 3358,2977,1707,1496,1369,1260,1169,1031,744,701,624 \mathrm{~cm}^{-1}$; MS (ESI) $\mathrm{m} / \mathrm{z}$ : 243, 498, $554(100)\left[(\mathrm{M}+\mathrm{Na})^{+}\right]$; HRMS (ESI) calcd for $\mathrm{C}_{32} \mathrm{H}_{37} \mathrm{NNaO}_{4} \mathrm{~S}\left[(\mathrm{M}+\mathrm{Na})^{+}\right]$554.2341, found 554.2361 .

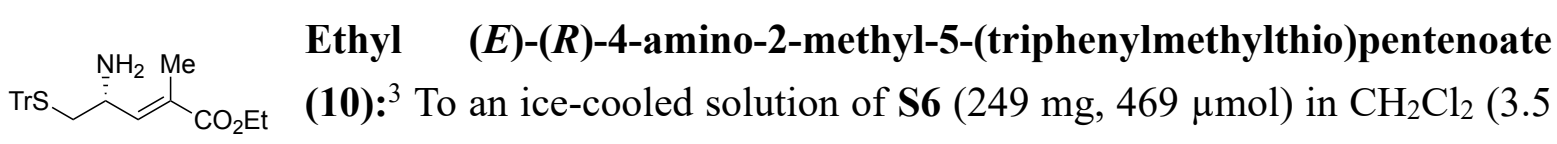
$\mathrm{mL})$ were added 2,6-lutidine $(328 \mu \mathrm{L}, 2.81 \mathrm{mmol})$ and trimethylsilyl trifluoromethanesulfonate $(550 \mu \mathrm{L}, 2.02 \mathrm{mmol})$, and the mixture was stirred at $0{ }^{\circ} \mathrm{C}$ for $3 \mathrm{~h}$. The mixture was diluted with methanol $(5 \mathrm{~mL})$ and water $(5 \mathrm{~mL})$ and extracted with $\mathrm{CHCl}_{3}(15$ $\mathrm{mL})$. The extracts were washed with brine $(10 \mathrm{~mL})$, dried, and concentrated to afford amine $\mathbf{1 0}$ (345 mg) as a pale yellow amorphous. The crude mixture was partially purified by flash chromatography to obtain the analytical sample. $[\alpha]_{\mathrm{D}}{ }^{24}-102.7\left(c 1.00, \mathrm{CHCl}_{3}\right) ;{ }^{1} \mathrm{H} \mathrm{NMR}(400$ $\left.\mathrm{MHz}, \mathrm{CDCl}_{3}\right) \delta 7.42(\mathrm{~d}, J=8.0 \mathrm{~Hz}, 6 \mathrm{H}), 7.31-7.21(\mathrm{~m}, 9 \mathrm{H}), 6.46(\mathrm{~d}, J=9.2 \mathrm{~Hz}, 1 \mathrm{H}), 4.08(\mathrm{q}$, $J=7.2 \mathrm{~Hz}, 2 \mathrm{H}), 2.94-2.89(\mathrm{~m}, 2 \mathrm{H}), 2.63(\mathrm{dd}, J=13.6,6.4 \mathrm{~Hz}, 1 \mathrm{H}), 1.59$ (s, 3H), $1.23(\mathrm{t}, J=$ $7.2 \mathrm{~Hz}, 3 \mathrm{H}) ;{ }^{13} \mathrm{C}$ NMR $\left(100 \mathrm{MHz}, \mathrm{CDCl}_{3}\right) \delta 167.3,154.2,144.0,134.6,133.0,129.4,128.2$, 127.1, 124.3, 67.6, 61.4, 48.8, 34.0, 19.8, 13.4; FT-IR (neat) v 2979, 1712, 1649, 1492, 1445, 1376, 1281, 1117, 1029, 751, 703, 633, $516 \mathrm{~cm}^{-1}$; MS (ESI) m/z: 165, 243 (100), 454 $\left[(\mathrm{M}+\mathrm{Na})^{+}\right]$; HRMS (ESI) calcd for $\mathrm{C}_{27} \mathrm{H}_{29} \mathrm{NNaO}_{2} \mathrm{~S}\left[(\mathrm{M}+\mathrm{Na})^{+}\right]$454.1817, found 454.1835.

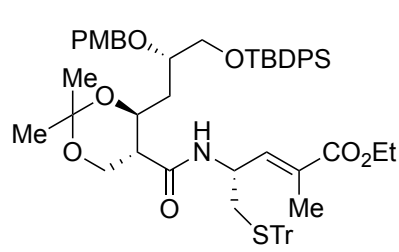

Ethyl $(R, E)-4-((4 S, 5 R)-4-((S)-2-(4-m e t h o x y b e n z y l o x y)-3-t e r t-b u-$ tyldiphenylsilyloxypropyl)-2,2-dimethyl-1,3-dioxane-5-carboxamido)-2-methyl-5-(tritylthio)pent-2-enoate (S7): To a solution of carboxylic acid $9(214 \mathrm{mg}, 361 \mu \mathrm{mol})$ and amine $10(202 \mathrm{mg}, 469$ $\mu \mathrm{mol})$ in $\mathrm{CH}_{2} \mathrm{Cl}_{2}(7 \mathrm{~mL})$ were added $N, N$-diisopropylethylamine $(124 \mu \mathrm{L}, 722 \mu \mathrm{mol})$ and HATU $(178 \mathrm{mg}, 469 \mu \mathrm{mol})$. After stirring at room temperature for 14 $\mathrm{h}$, the mixture was concentrated. The residue was purified by flash chromatography $\left(\mathrm{SiO}_{2} 25\right.$ $\mathrm{g}$, hexane/EtOAc $=3: 1$ to EtOAc:methanol $=10: 1)$ to afford $\mathbf{S 7}(279 \mathrm{mg}, 277 \mu \mathrm{mol}, 77 \%)$ as a pale yellow amorphous. $[\alpha]_{\mathrm{D}}{ }^{25}-13.4\left(c 1.39, \mathrm{CHCl}_{3}\right) ;{ }^{1} \mathrm{H} \mathrm{NMR}\left(400 \mathrm{MHz}, \mathrm{CDCl}_{3}\right) \delta 7.69$ $7.65(\mathrm{~m}, 4 \mathrm{H}), 7.45-7.31(\mathrm{~m}, 13 \mathrm{H}), 7.24-7.13(\mathrm{~m}, 12 \mathrm{H}), 6.82(\mathrm{~d}, J=8.5 \mathrm{~Hz}, 2 \mathrm{H}), 5.57(\mathrm{~d}, J=$ $7.3 \mathrm{~Hz}, 1 \mathrm{H}), 4.64(\mathrm{~d}, J=11.2 \mathrm{~Hz}, 1 \mathrm{H}), 4.58-4.51(\mathrm{~m}, 1 \mathrm{H}), 4.36(\mathrm{~d}, J=11.2 \mathrm{~Hz}, 1 \mathrm{H}), 4.21-4.09$ 
$(\mathrm{m}, 4 \mathrm{H}), 4.01-3.95(\mathrm{~m}, 1 \mathrm{H}), 3.78(\mathrm{~s}, 3 \mathrm{H}), 3.76-3.69(\mathrm{~m}, 2 \mathrm{H}), 3.56-3.54(\mathrm{~m}, 1 \mathrm{H}), 2.33(\mathrm{~d}, J=$ $6.6 \mathrm{~Hz}, 2 \mathrm{H}), 2.17(\mathrm{dt}, J=10.4,4.8 \mathrm{~Hz}, 1 \mathrm{H}), 1.72(\mathrm{~d}, J=1.4 \mathrm{~Hz}, 3 \mathrm{H}), 1.31(\mathrm{~s}, 6 \mathrm{H}), 1.26(\mathrm{t}, J=$ $7.0 \mathrm{~Hz}, 3 \mathrm{H}), 1.04$ (s, 9H); ${ }^{13} \mathrm{C} \mathrm{NMR}\left(100 \mathrm{MHz}, \mathrm{CDCl}_{3}\right) \delta 169.6,167.4,159.0,144.4,144.3$, 138.7, 135.6, 135.6, 133.6, 133.5, 131.1, 130.4, 129.6, 129.5, 129.5, 129.3, 128.0, 127.6, 126.8, $120.1,113.7,98.4,77.2,75.7,72.2,67.2,67.1,67.0,62.0,60.8,55.2,48.7,46.9,37.1,35.7$, 29.3, 26.8, 24.4, 19.2, 19.1, 14.2, 12.9, 12.8; FT-IR (neat) v 3333, 2935, 1709, 1667, 1517 , 1377, 1254, 1105, 818, 741, 701, $505 \mathrm{~cm}^{-1}$; MS (FAB) m/z: 121, 154 (100), 243, 307, 460, 762, 884, $1006\left(\mathrm{M}+\mathrm{H}^{+}\right)$; HRMS (FAB) calcd for $\mathrm{C}_{61} \mathrm{H}_{72} \mathrm{NO}_{8} \mathrm{SSi}\left(\mathrm{M}+\mathrm{H}^{+}\right)$1006.4748, found 1006.4747 .

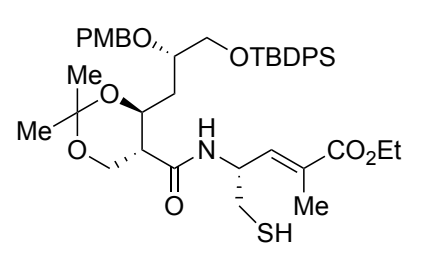

Ethyl $(R, E)-4-((4 S, 5 R)-4-((S)-2-(4-m e t h o x y b e n z y l o x y)-3-t e r t-b u-$ tyldiphenylsilyloxypropyl)-2,2-dimethyl-1,3-dioxane-5-carboxamido)-5-mercapto-2-methylpent-2-enoate (S8): To an ice-cooled solution of $\mathbf{S 7}(410 \mathrm{mg}, 407 \mu \mathrm{mol})$ in EtOAc $(4.88 \mathrm{~mL})$ and ethanol $(3.26 \mathrm{~mL})$ was added mercury(II) acetate $(161 \mathrm{mg}, 504 \mu \mathrm{mol})$, and the mixture was stirred at room temperature for $2.5 \mathrm{~h}$. To this mixture was added sodium borohydride $(30.8 \mathrm{mg}, 814 \mu \mathrm{mol})$. After stirring at room temperature for $3 \mathrm{~h}$, the mixture was filtered through Celite pad and concentrated. The residue was purified by flash chromatography $\left(\mathrm{SiO}_{2} 10 \mathrm{~g}\right.$, hexane/EtOAc $=3: 1$ to only EtOAc) to afford $\mathbf{S 8}(286 \mathrm{mg}, 375 \mu \mathrm{mol}, 92 \%)$ as a colorless amorphous. $[\alpha]_{\mathrm{D}}{ }^{23}-25.0\left(c 1.06, \mathrm{CHCl}_{3}\right) ;{ }^{1} \mathrm{H} \mathrm{NMR}\left(400 \mathrm{MHz}, \mathrm{CDCl}_{3}\right) \delta 7.68-7.66$ $(\mathrm{m}, 4 \mathrm{H}), 7.43-7.31(\mathrm{~m}, 6 \mathrm{H}), 7.26(\mathrm{~d}, J=8.7 \mathrm{~Hz}, 2 \mathrm{H}), 6.86(\mathrm{~d}, J=8.7 \mathrm{~Hz}, 2 \mathrm{H}), 6.45(\mathrm{~d}, J=9.0$ $\mathrm{Hz}, 1 \mathrm{H}), 5.93$ (d, $J=8.1 \mathrm{~Hz}, 1 \mathrm{H}), 4.87-4.71(\mathrm{~m}, 1 \mathrm{H}), 4.66(\mathrm{~d}, J=11.4 \mathrm{~Hz}, 1 \mathrm{H}), 4.40(\mathrm{~d}, J=$ $11.4 \mathrm{~Hz}, 1 \mathrm{H}), 4.29-4.01(\mathrm{~m}, 5 \mathrm{H}), 3.89-3.67(\mathrm{~m}, 5 \mathrm{H}), 3.80(\mathrm{~s}, 3 \mathrm{H}), 2.59-2.54(\mathrm{~m}, 2 \mathrm{H}), 2.28$ (dt, $J=9.9,4.8 \mathrm{~Hz}, 1 \mathrm{H}), 1.90(\mathrm{~s}, 3 \mathrm{H}), 1.77-1.60(\mathrm{~m}, 1 \mathrm{H}), 1.35$ (s, 3H), 1.32 (s, 3H), 1.29 (t, $J=7.2$ $\mathrm{Hz}, 3 \mathrm{H}), 1.05$ (s, 9H); ${ }^{13} \mathrm{C} \mathrm{NMR}\left(100 \mathrm{MHz}, \mathrm{CDCl}_{3}\right) \delta 169.8,167.4,159.1,137.3,135.6,133.5$, $131.9,131.0,129.5,127.7,113.8,113.8,98.5,77.2$, 75.7, 72.2, 67.1, 67.0, 66.8, 62.0, 61.0, $55.3,48.9,48.7,37.4,29.3,28.8,26.8,19.2,14.2,13.3$; FT-IR (neat) v 3313, 2928, 1711, 1648, $1518,1377,1255,1106,818,703 \mathrm{~cm}^{-1}$; MS (ESI) $\mathrm{m} / z$ : $786(100)\left[(\mathrm{M}+\mathrm{Na})^{+}\right], 1550$; HRMS (ESI) calcd for $\mathrm{C}_{42} \mathrm{H}_{57} \mathrm{NNaO}_{8} \mathrm{SSi}\left[(\mathrm{M}+\mathrm{Na})^{+}\right]$786.3471, found 786.3506.

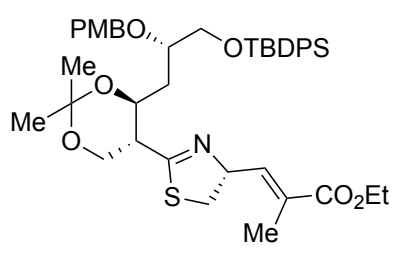

Ethyl (2E)-3-((R)-2-((4S,5R)-4-((S)-2-(4-methoxybenzyloxy)-3tert-butyldiphenylsilyloxypropyl)-2,2-dimethyl-1,3-dioxan-5-yl)4,5-dihydrothiazol-4-yl)-2-methylacrylate (S9): To a solution of S8 $(289 \mathrm{mg}, 379 \mu \mathrm{mol})$ in toluene $(38 \mathrm{~mL})$ was added hexaammonium heptamolybdate tetrahydrate (49 $\mathrm{mg}, 39.6 \mu \mathrm{mol})$, and the mixture was stirred at $140{ }^{\circ} \mathrm{C}$ for $4 \mathrm{~h}$. To this mixture was added hexaammonium heptamolybdate tetrahydrate ( $49 \mathrm{mg}, 39.6 \mu \mathrm{mol}$ ) again. After stirring at $140{ }^{\circ} \mathrm{C}$ for $19 \mathrm{~h}$, the mixture was concentrated. The residue was purified by flash chromatography $\left(\mathrm{SiO}_{2} 30 \mathrm{~g}\right.$, 
hexane/EtOAc $=4: 1)$ to give $\mathbf{S 9}(261 \mathrm{mg}, 350 \mu \mathrm{mol}, 92 \%)$ as a colorless amorphous. $[\alpha]_{\mathrm{D}}{ }^{23}$ +6.7 (c 1.16, $\left.\mathrm{CHCl}_{3}\right) ;{ }^{1} \mathrm{H}$ NMR (400 MHz, $\left.\mathrm{CDCl}_{3}\right) \delta$ 7.69-7.66 (m, 4H), 7.41-7.34 (m, 6H), $7.23(\mathrm{~d}, J=8.8 \mathrm{~Hz}, 2 \mathrm{H}), 6.83(\mathrm{~d}, J=8.8 \mathrm{~Hz}, 2 \mathrm{H}), 6.70(\mathrm{~d}, J=8.8 \mathrm{~Hz}, 1 \mathrm{H}), 5.14(\mathrm{q}, J=9.3 \mathrm{~Hz}$, $1 \mathrm{H}), 4.63(\mathrm{~d}, J=11.0 \mathrm{~Hz}, 1 \mathrm{H}), 4.41(\mathrm{~d}, J=11.0 \mathrm{~Hz}, 1 \mathrm{H}), 4.31-4.25(\mathrm{~m}, 1 \mathrm{H}), 4.18$ (q, $J=6.9$ $\mathrm{Hz}, 2 \mathrm{H}), 4.07-4.01(\mathrm{~m}, 1 \mathrm{H}), 3.91$ (dd, $J=10.9,4.8 \mathrm{~Hz}, 1 \mathrm{H}), 3.85-3.74(\mathrm{~m}, 1 \mathrm{H}), 3.79(\mathrm{~s}, 3 \mathrm{H})$, 3.70-3.55 (m, 2H), $3.40(\mathrm{dd}, J=10.8,8.8 \mathrm{~Hz}, 1 \mathrm{H}), 2.95(\mathrm{dd}, J=10.8,8.8 \mathrm{~Hz}, 1 \mathrm{H}), 2.73(\mathrm{dt}, J$ $=10.9,4.8 \mathrm{~Hz}, 1 \mathrm{H}), 1.92(\mathrm{~s}, 3 \mathrm{H}), 1.86-1.79(\mathrm{~m}, 1 \mathrm{H}), 1.40(\mathrm{~s}, 3 \mathrm{H}), 1.37(\mathrm{~s}, 3 \mathrm{H}), 1.32-1.21(\mathrm{~m}$, $1 \mathrm{H}), 1.26(\mathrm{t}, J=6.9 \mathrm{~Hz}, 3 \mathrm{H}), 1.06(\mathrm{~s}, 9 \mathrm{H}) ;{ }^{13} \mathrm{C} \mathrm{NMR}\left(100 \mathrm{MHz}, \mathrm{CDCl}_{3}\right) \delta 169.4,167.5,159.0$, 139.6, 135.7, 135.6, 134.8, 133.6, 133.6, 131.2, 130.0, 129.5, 129.1, 129.1, 127.7, 127.6, 113.7, 98.6, 75.3, 74.5, 72.3, 68.5, 66.9, 63.0, 60.8, 55.2, 46.4, 37.9, 36.8, 29.4, 26.9, 26.5, 19.3, 19.2, 14.2, 13.1; FT-IR (neat) v 2933, 1713, 1610, 1511, 1463, 1375, 1251, 1106, 818, $701 \mathrm{~cm}^{-1}$; MS (ESI) $m / z: 746,768(100)\left[(\mathrm{M}+\mathrm{Na})^{+}\right], 784$; HRMS (ESI) calcd for $\mathrm{C}_{42} \mathrm{H}_{55} \mathrm{NNaO}_{7} \mathrm{SSi}\left[(\mathrm{M}+\mathrm{Na})^{+}\right]$ 768.3366, found 768.3405 .

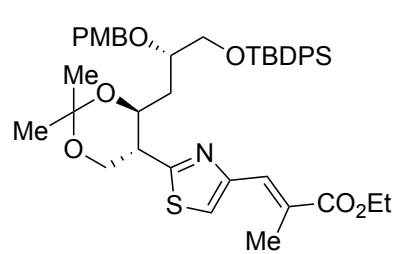

Ethyl (E)-3-(2-((4S,5R)-4-((S)-2-(4-methoxybenzyloxy)-3-tert-butyldiphenylsilyloxypropyl)-2,2-dimethyl-1,3-dioxan-5-yl)thiazol4-yl)-2-methylacrylate (17): To a solution of $\mathbf{S 9}(12.0 \mathrm{mg}, 16.1$ $\mu \mathrm{mol})$ in hexane $(4.0 \mathrm{~mL})$ was added activated manganese (IV) dioxide (218 $\mathrm{mg}, 2.51 \mathrm{mmol}$ ), and the mixture was stirred at room temperature for $22.5 \mathrm{~h}$. The mixture was filtered through Celite pad and concentrated. The residue was purified by flash chromatography $\left(\mathrm{SiO}_{2} 1.2 \mathrm{~g}\right.$, hexane/EtOAc $\left.=4: 1\right)$ to give $17(9.4$ $\mathrm{mg}, 12.6 \mu \mathrm{mol}, 79 \%)$ as a colorless amorphous. $[\alpha]_{\mathrm{D}}{ }^{23}-2.5\left(c 1.00, \mathrm{CHCl}_{3}\right) ;{ }^{1} \mathrm{H} \mathrm{NMR}(400$ $\left.\mathrm{MHz}, \mathrm{CDCl}_{3}\right) \delta$ 7.66-7.64 (m, 4H), $7.60(\mathrm{~s}, 1 \mathrm{H}), 7.42-7.32(\mathrm{~m}, 6 \mathrm{H}), 7.27(\mathrm{~s}, 1 \mathrm{H}), 7.19(\mathrm{~d}, J=$ $8.8 \mathrm{~Hz}, 2 \mathrm{H}), 6.83(\mathrm{~d}, J=8.8 \mathrm{~Hz}, 2 \mathrm{H}), 4.58(\mathrm{~d}, J=11.2 \mathrm{~Hz}, 1 \mathrm{H}), 4.41-4.35(\mathrm{~m}, 1 \mathrm{H}), 4.36(\mathrm{~d}, J$ $=11.2 \mathrm{~Hz}, 1 \mathrm{H}), 4.26(\mathrm{q}, J=7.2 \mathrm{~Hz}, 2 \mathrm{H}), 4.19-4.13(\mathrm{~m}, 1 \mathrm{H}), 3.99(\mathrm{dd}, J=11.2,5.2 \mathrm{~Hz}, 1 \mathrm{H})$, $3.80(\mathrm{~s}, 3 \mathrm{H}) 3.79-3.74(\mathrm{~m}, 1 \mathrm{H}), 3.65-3.57(\mathrm{~m}, 2 \mathrm{H}), 3.19(\mathrm{dt}, J=11.2,5.2 \mathrm{~Hz}, 1 \mathrm{H}), 2.30(\mathrm{~s}, 3 \mathrm{H})$, $1.69-1.52$ (m, 1H), 1.46 (s, 3H), 1.43 (s, 3H), 1.33 (t, $J=7.2 \mathrm{~Hz}, 3 \mathrm{H}), 1.28-1.25$ (m, 1H), 1.02 $(\mathrm{s}, 9 \mathrm{H}) ;{ }^{13} \mathrm{C} \mathrm{NMR}\left(100 \mathrm{MHz}, \mathrm{CDCl}_{3}\right) \delta 188.9,168.7,166.3,159.0,152.5,141.2,135.6,133.5$, $133.5,132.9,131.1,130.2,129.6,129.3,129.1,127.6,120.7,113.7,98.6,77.3,75.1,72.2,69.5$, $66.7,64.5,60.9,55.2,45.7,42.8,41.1,36.6,31.4,29.4,26.8,19.2,19.1,14.4$, 14.3; FT-IR (neat) v 2925, 2857, 1705, 1617, 1509, 1464, 1375, 1244, 1108, $817 \mathrm{~cm}^{-1}$; MS (ESI) m/z: 766 (100) $\left[(\mathrm{M}+\mathrm{Na})^{+}\right]$; HRMS (ESI) calcd for $\mathrm{C}_{42} \mathrm{H}_{53} \mathrm{NNaO}_{7} \mathrm{SSi}\left[(\mathrm{M}+\mathrm{Na})^{+}\right] 766.3209$, found 766.3207 .

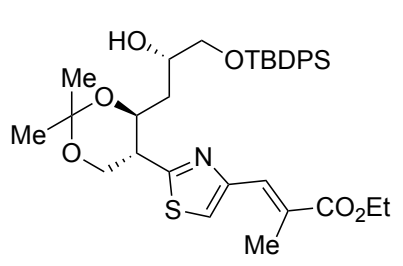

Ethyl (E)-3-(2-((4S,5R)-4-((S)-2-hydroxy-3-tert-butyldiphenylsilyloxypropyl)-2,2-dimethyl-1,3-dioxan-5-yl)thiazol-4-yl)-2-methylacrylate (S10): To a solution of $17(128.5 \mathrm{mg}, 173 \mu \mathrm{mol})$ in $\mathrm{CH}_{2} \mathrm{Cl}_{2}$ $(55 \mathrm{~mL})$ and phosphate buffer $\left(\mathrm{KH}_{2} \mathrm{PO}_{4} / \mathrm{Na}_{2} \mathrm{HPO}_{4}, \mathrm{pH} 7.0,2.75 \mathrm{~mL}\right)$ 
was added 2,3-dichloro-5,6-dicyano- $p$-benzoquinone (235.4 $\mathrm{mg}, 1.04 \mathrm{mmol}$ ), and the mixture was stirred at room temperature for $6 \mathrm{~h}$. The mixture was diluted with saturated $\mathrm{NaHCO}_{3}(50$ $\mathrm{mL})$ and saturated $\mathrm{Na}_{2} \mathrm{~S}_{2} \mathrm{O}_{3}(50 \mathrm{~mL})$, and extracted with EtOAc $(200 \mathrm{~mL})$. The extracts were washed with brine $(100 \mathrm{~mL})$, dried and concentrated. The residue was purified by flash chromatography $\left(\mathrm{SiO}_{2} 5 \mathrm{~g}\right.$, hexane/EtOAc $\left.=10: 1\right)$ to afford $\mathbf{S 1 0}(103.0 \mathrm{mg}, 165 \mu \mathrm{mol}, 96 \%)$ as a colorless oil. $[\alpha]_{\mathrm{D}}{ }^{24}-34.2\left(c 1.00, \mathrm{CHCl}_{3}\right) ;{ }^{1} \mathrm{H} \mathrm{NMR}\left(400 \mathrm{MHz}, \mathrm{CDCl}_{3}\right) \delta 7.62-7.59(\mathrm{~m}$, 4H), 7.59 (s, 1H), 7.42-7.34 (m, 6H), $7.29(\mathrm{~s}, 1 \mathrm{H}), 4.50$ (dt, $J=9.6,2.4 \mathrm{~Hz}, 1 \mathrm{H}), 4.26$ (q, $J=$ $7.0 \mathrm{~Hz}, 2 \mathrm{H}), 4.17$ (t, $J=11.6 \mathrm{~Hz}, 1 \mathrm{H}), 4.00(\mathrm{dd}, J=11.6,5.2 \mathrm{~Hz}, 1 \mathrm{H}), 3.98-3.92(\mathrm{~m}, 1 \mathrm{H}), 3.63$ $(\mathrm{dd}, J=10.4,4.4 \mathrm{~Hz}, 1 \mathrm{H}), 3.48(\mathrm{dd}, J=10.4,6.6 \mathrm{~Hz}, 1 \mathrm{H}), 3.27(\mathrm{dt}, J=9.6,5.2 \mathrm{~Hz}, 1 \mathrm{H}), 2.71$ $(\mathrm{d}, J=4.4 \mathrm{~Hz}, 1 \mathrm{H}), 2.32(\mathrm{~s}, 3 \mathrm{H}), 1.70-1.65(\mathrm{~m}, 1 \mathrm{H}), 1.57-1.50(\mathrm{~m}, 1 \mathrm{H}), 1.55(\mathrm{~s}, 3 \mathrm{H}), 1.40$ (s, $3 \mathrm{H}), 1.34(\mathrm{t}, J=7.0 \mathrm{~Hz}, 3 \mathrm{H}), 0.98(\mathrm{~s}, 9 \mathrm{H}) ;{ }^{13} \mathrm{C} \mathrm{NMR}\left(100 \mathrm{MHz}, \mathrm{CDCl}_{3}\right) \delta 168.7,166.2,152.6$, $135.5,135.5,133.2,133.1,130.2$, 129.7, 129.5, 127.7, 120.6, 98.8, 70.5, 68.4, 67.8, 64.5, 60.9, 45.1, 36.5, 29.3, 26.8, 19.2, 19.1, 14.4, 14.3, 12.5, 4.3; FT-IR (neat) v 3516, 2954, 2249, 1897 , 1709, 1474, 1132, 741, 611, $521 \mathrm{~cm}^{-1}$; MS (FAB) $\mathrm{m} / z:$ 75, 135 (100), 199, 310, 430, 478, 508, $566,624\left[(\mathrm{M}+\mathrm{H})^{+}\right]$; HRMS $(\mathrm{FAB})$ calcd for $\mathrm{C}_{34} \mathrm{H}_{46} \mathrm{NO}_{6} \mathrm{SSi}\left[(\mathrm{M}+\mathrm{H})^{+}\right]$622.2816, found 622.2813 .

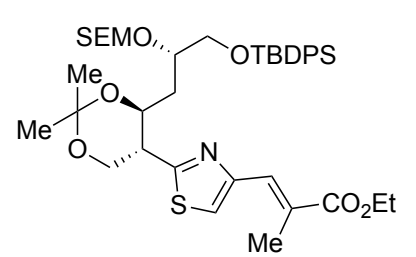

Ethyl (E)-3-(2-((4S,5R)-4-((S)-2-((2-(trimethylsilyl)ethoxy)methoxy)-3-tert-butyldiphenylsilyloxypropyl)-2,2-dimethyl-1,3-dioxan-5-yl)thiazol-4-yl)-2-methylacrylate (S11): To a solution of S10 (1.68 g, $2.69 \mathrm{mmol})$ in $\mathrm{CH}_{2} \mathrm{Cl}_{2}(33 \mathrm{~mL})$ were added $\mathrm{N}, \mathrm{N}$ diisopropylethylamine $(2.3 \mathrm{~mL}, \quad 13.1 \mathrm{mmol})$ and 2(trimethylsilyl)ethoxymethyl chloride $(1.1 \mathrm{~mL}, 6.5 \mathrm{mmol})$, and the mixture was stirred at room temperature for 5 days. The reaction was quenched with saturated $\mathrm{NH}_{4} \mathrm{Cl}(20 \mathrm{~mL})$ and the mixture was extracted with EtOAc $(100 \mathrm{~mL})$. The extracts were washed with brine $(50 \mathrm{ml})$, dried, and concentrated. The residue was purified by flash chromatography $\left(\mathrm{SiO}_{2} 100 \mathrm{~g}\right.$, hexane/EtOAc $=5: 1)$ to give $\mathbf{S 1 1}(2.02 \mathrm{~g}, 2.69 \mathrm{mmol}, 100 \%)$ as a colorless oil. $[\alpha]_{\mathrm{D}}{ }^{28}-9.8(c$ 1.93, $\mathrm{CHCl}_{3}$ ); ${ }^{1} \mathrm{H}$ NMR (400 MHz, $\left.\mathrm{CDCl}_{3}\right) \delta 7.64$ (s, $\left.1 \mathrm{H}\right), 7.62$ (d, $\left.J=6.4 \mathrm{~Hz}, 4 \mathrm{H}\right), 7.41-7.34$ $(\mathrm{m}, 6 \mathrm{H}), 7.29(\mathrm{~s}, 1 \mathrm{H}), 4.73(\mathrm{~d}, J=6.8 \mathrm{~Hz}, 1 \mathrm{H}), 4.67(\mathrm{~d}, J=6.8 \mathrm{~Hz}, 1 \mathrm{H}), 4.35(\mathrm{dt}, J=10.6,2.8$ $\mathrm{Hz}, 1 \mathrm{H}), 4.26(\mathrm{q}, J=7.2 \mathrm{~Hz}, 2 \mathrm{H}), 4.16(\mathrm{t}, J=12.0 \mathrm{~Hz}, 1 \mathrm{H}), 4.01(\mathrm{dd}, J=12.0,5.2 \mathrm{~Hz}, 1 \mathrm{H})$, 3.87-3.84 (m, 1H), 3.63-3.57 (m, 2H), $3.54(\mathrm{dd}, J=8.8,2.2 \mathrm{~Hz}, 2 \mathrm{H}), 3.22(\mathrm{dt}, J=10.6,5.2 \mathrm{~Hz}$, $1 \mathrm{H}), 2.32(\mathrm{~s}, 3 \mathrm{H}), 1.64-1.62(\mathrm{~m}, 2 \mathrm{H}), 1.56(\mathrm{~s}, 3 \mathrm{H}), 1.43(\mathrm{~s}, 3 \mathrm{H}), 1.34(\mathrm{t}, J=7.2 \mathrm{~Hz}, 3 \mathrm{H}), 1.00$ (s, 9H), 0.83 (dt, $J=8.8,2.2 \mathrm{~Hz}, 2 \mathrm{H}),-0.04(\mathrm{~s}, 9 \mathrm{H}) ;{ }^{13} \mathrm{C} \mathrm{NMR}\left(100 \mathrm{MHz}, \mathrm{CDCl}_{3}\right) \delta 168.7$, 166.4 , 152.6, 135.6, 135.6, 133.6, 133.5, 130.2, 129.6, 129.4, 127.6, 120.7, 98.7, 95.3, 94.3, 91.6, 74.6, 69.6, 66.9, 65.7, 65.3, 64.9, 64.5, 60.9, 45.7, 36.8, 29.4, 26.8, 19.2, 19.2 , 18.1, 18.0, 14.4, 14.3, -1.4, -1.4; FT-IR (neat) v 2952, 1708, 1377, 1247, 1111, 934, 835, 702, 613, 507 $\mathrm{cm}^{-1}$; MS (ESI) $\mathrm{m} / z:$ 143, 187, 270, 300, 329, 414, 451, 518, 558, 637, 695, 776 (100) $\left[(\mathrm{M}+\mathrm{Na})^{+}\right]$, 975; HRMS (ESI) calcd for $\mathrm{C}_{40} \mathrm{H}_{59} \mathrm{NNaO}_{7} \mathrm{SSi}_{2}\left[(\mathrm{M}+\mathrm{Na})^{+}\right] 776.3448$, found 
776.3494.

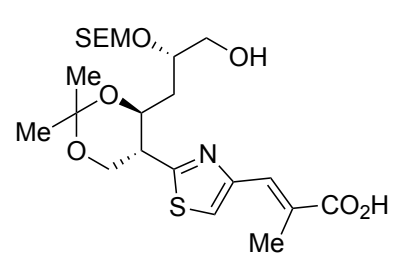

(E)-3-(2-((4S,5R)-4-((S)-2-((2-(Trimethylsilyl)ethoxy)methoxy)3-hydroxypropyl)-2,2-dimethyl-1,3-dioxan-5-yl)thiazol-4-yl)-2methylacrylic acid (18): To an ice-cooled S11 (2.02 g, $2.69 \mathrm{mmol})$ was added $10 \% \mathrm{NaOH}$ in methanol $(27 \mathrm{~mL})$, and the mixture was stirred at $75^{\circ} \mathrm{C}$ for $24 \mathrm{~h}$. The reaction was quenched with saturated $\mathrm{NH}_{4} \mathrm{Cl}(100 \mathrm{~mL})$ and the mixture was extracted with EtOAc $(400 \mathrm{~mL})$. The extracts were washed with brine $(100 \mathrm{ml})$, dried, and concentrated. The residue was purified by column chromatography $\left(\mathrm{SiO}_{2} 60 \mathrm{~g}\right.$, hexane/EtOAc $=2: 1$ to only EtOAc to $\mathrm{CHCl}_{3} /$ methanol $\left.=10: 1\right)$ to give 18 (1.32 g, $2.69 \mathrm{mmol}, 100 \%)$ as a yellow oil. $[\alpha]_{\mathrm{D}}{ }^{28}-0.97\left(c 1.19, \mathrm{CHCl}_{3}\right) ;{ }^{1} \mathrm{H} \mathrm{NMR}$ $\left(400 \mathrm{MHz}, \mathrm{CDCl}_{3}\right) \delta 7.70(\mathrm{~s}, 1 \mathrm{H}), 7.35(\mathrm{~s}, 1 \mathrm{H}), 4.77$ (d, J=7.2 Hz, 1H), 4.62 (d, J= 7.2 Hz, $1 \mathrm{H}), 4.38(\mathrm{t}, J=12.4 \mathrm{~Hz}, 1 \mathrm{H}), 4.15(\mathrm{t}, J=11.6 \mathrm{~Hz}, 1 \mathrm{H}), 4.01(\mathrm{dd}, J=11.6,5.2 \mathrm{~Hz}, 1 \mathrm{H}), 3.79$ $3.76(\mathrm{~m}, 1 \mathrm{H}), 3.76-3.72(\mathrm{~m}, 1 \mathrm{H}), 3.57-3.49(\mathrm{~m}, 2 \mathrm{H}), 3.42(\mathrm{dd}, J=13.6,6.8 \mathrm{~Hz}, 1 \mathrm{H}), 3.23(\mathrm{dt}$, $J=12.4,5.2 \mathrm{~Hz}, 1 \mathrm{H}), 2.35$ (s, 3H), 1.69 (t, $J=12.4 \mathrm{~Hz}, 1 \mathrm{H}), 1.56(\mathrm{~s}, 3 \mathrm{H}), 1.56-1.50$ (m, $1 \mathrm{H})$, 1.45 (s, 3H), $0.93(\mathrm{ddd}, J=13.6,6.8,2.4 \mathrm{~Hz}, 2 \mathrm{H}), 0.00(\mathrm{~s}, 9 \mathrm{H}) ;{ }^{13} \mathrm{C} \mathrm{NMR}\left(100 \mathrm{MHz}, \mathrm{CDCl}_{3}\right)$ $\delta 173.3,166.4,152.4,131.9,128.4,121.8,98.8,95.7,79.1,69.4,66.2,65.9,64.6,45.5,36.1$, 29.3, 19.2, 18.1, 14.1, -1.5; FT-IR (neat) v 3435, 2950, 1697, 1634, 1381, 1256, 1204, 1112 , $1056,840 \mathrm{~cm}^{-1}$; MS (ESI) $m / z: 445,510(100)\left[(\mathrm{M}+\mathrm{Na})^{+}\right], 586,662,783,800,821,837$; HRMS (ESI) calcd for $\mathrm{C}_{22} \mathrm{H}_{37} \mathrm{NNaO}_{7} \mathrm{SSi}\left[(\mathrm{M}+\mathrm{Na})^{+}\right]$510.1958, found 510.1923.

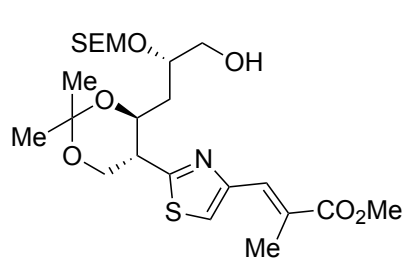

Methyl (E)-3-(2-((4S,5R)-4-((S)-2-((2-(trimethylsilyl)ethoxy)methoxy)-3-hydroxypropyl)-2,2-dimethyl-1,3-dioxan-5-yl)thiazol4-yl)-2-methylacrylate (S12): To a solution of $\mathbf{1 8}(0.81 \mathrm{~g}, 1.67$ $\mathrm{mmol})$ in methanol $(11.1 \mathrm{~mL})$ was added dropwise trimethylsilyl diazomethane (0.6 $\mathrm{M}$ in hexane, $16.7 \mathrm{~mL}, 10.0 \mathrm{mmol})$, and the mixture was stirred at room temperature for $1 \mathrm{~h}$. The reaction was quenched with acetic acid $(2.5 \mathrm{~mL})$ and the mixture was concentrated. The residue was purified by column chromatography $\left(\mathrm{SiO}_{2} 60 \mathrm{~g}\right.$, hexane/EtOAc $\left.=3: 1\right)$ to afford $\mathbf{S 1 2}(0.66 \mathrm{~g}, 1.31 \mathrm{mmol}, 79 \%)$ as a yellow oil. $[\alpha]_{\mathrm{D}}{ }^{27}+3.1\left(c 2.09, \mathrm{CHCl}_{3}\right) ;{ }^{1} \mathrm{H} \mathrm{NMR}\left(400 \mathrm{MHz}, \mathrm{CDCl}_{3}\right) \delta 7.60(\mathrm{~s}, 1 \mathrm{H}), 7.30(\mathrm{~s}$, $1 \mathrm{H}), 4.75(\mathrm{~d}, J=7.0 \mathrm{~Hz}, 1 \mathrm{H}), 4.61(\mathrm{~d}, J=7.0 \mathrm{~Hz}, 1 \mathrm{H}), 4.36(\mathrm{dt}, J=10.4,2.4 \mathrm{~Hz}, 1 \mathrm{H}), 4.14$ (t, $J=11.6 \mathrm{~Hz}, 1 \mathrm{H}), 4.00(\mathrm{dd}, J=11.6,5.2 \mathrm{~Hz}, 1 \mathrm{H}), 3.80(\mathrm{~s}, 3 \mathrm{H}), 3.74(\mathrm{dt}, J=10.6,6.5 \mathrm{~Hz}, 2 \mathrm{H})$, $3.53(\mathrm{dd}, J=10.0,6.8 \mathrm{~Hz}, 1 \mathrm{H}), 3.51(\mathrm{dd}, J=10.0,6.8 \mathrm{~Hz}, 1 \mathrm{H}), 3.41(\mathrm{dd}, J=12.4,6.8 \mathrm{~Hz}, 2 \mathrm{H})$, $3.22(\mathrm{dt}, J=10.4,5.2 \mathrm{~Hz}, 1 \mathrm{H}), 2.34(\mathrm{~s}, 3 \mathrm{H}), 1.68(\mathrm{dt}, J=12.4,2.4 \mathrm{~Hz}, 1 \mathrm{H}), 1.55(\mathrm{~s}, 3 \mathrm{H}), 1.50$ $(\mathrm{dt}, J=10.4,3.2 \mathrm{~Hz}, 1 \mathrm{H}), 1.44(\mathrm{~s}, 3 \mathrm{H}), 0.93$ (ddd, $J=10.6,6.5,4.0 \mathrm{~Hz}, 2 \mathrm{H}), 0.00(\mathrm{~s}, 9 \mathrm{H}) ;{ }^{13} \mathrm{C}$ NMR $\left(100 \mathrm{MHz}, \mathrm{CDCl}_{3}\right) \delta 169.2,166.3,152.6,130.3,129.2,120.9,100.6,98.8,95.7,79.0$, 69.4, 66.2, 65.8, 64.6, 52.1, 45.5, 36.1, 29.3, 19.2, 18.1, 14.3, -1.5; FT-IR (neat) v 3459, 2950, 1711, 1636, 1438, 1380, 1253, 1059, 843, $765 \mathrm{~cm}^{-1}$; MS (ESI) $m / z: 147,252,299,336,413$, 
461, $524(100)\left[(\mathrm{M}+\mathrm{Na})^{+}\right], 589,634,777,972$; HRMS (ESI) calcd for $\mathrm{C}_{23} \mathrm{H}_{39} \mathrm{NNaO}_{7} \mathrm{SSi}$ $\left[(\mathrm{M}+\mathrm{Na})^{+}\right]$524.2114, found 524.2099.

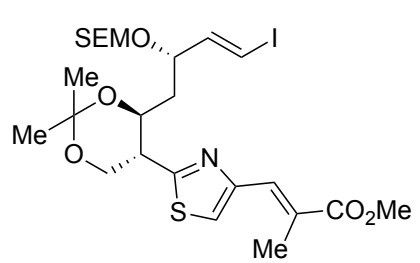

(2E)-Methyl 3-(2-((4S,5R)-4-((S,E)-2-((2-(trimethylsilyl)ethoxy)methoxy)-4-iodobut-3-enyl)-2,2-dimethyl-1,3-dioxan-5-yl)thiazol-4-yl)-2-methylacrylate (7): To a solution of oxalyl chloride $(0.27 \mathrm{~mL}, 3.17 \mathrm{mmol})$ in $\mathrm{CH}_{2} \mathrm{Cl}_{2}(10 \mathrm{~mL})$ was added dropwise the solution of DMSO $(0.45 \mathrm{~mL}, 6.33 \mathrm{mmol})$ in $\mathrm{CH}_{2} \mathrm{Cl}_{2}(1.0 \mathrm{~mL})$ at -

$78^{\circ} \mathrm{C}$. After stirring at $-78^{\circ} \mathrm{C}$ for $30 \mathrm{~min}$, a solution of $\mathbf{S 1 2}(0.79 \mathrm{~g}, 1.58 \mathrm{mmol})$ in $\mathrm{CH}_{2} \mathrm{Cl}_{2}(4.8$ $\mathrm{mL}$ ) was added, and the mixture was stirred at $-78{ }^{\circ} \mathrm{C}$ for $1 \mathrm{~h}$. Then triethylamine $(1.77 \mathrm{~mL}$, $12.7 \mathrm{mmol}$ ) was added, and stirring was continued at $0{ }^{\circ} \mathrm{C}$ for $7 \mathrm{~h}$. The reaction was quenched with saturated $\mathrm{NH}_{4} \mathrm{Cl}(10 \mathrm{~mL})$, and the mixture was extracted with EtOAc $(50 \mathrm{~mL})$. The extracts were washed with brine $(20 \mathrm{~mL})$, dried, and concentrated. The residue was purified by column chromatography $\left(\mathrm{SiO}_{2} 40 \mathrm{~g}\right.$, hexane/EtOAc $\left.=3: 1\right)$ to give aldehyde $(0.73 \mathrm{~g}, 1.47 \mathrm{mmol}$, 93\%) as a pale yellow oil.

To an ice-cooled solution of chromium(II) chloride $(1.21 \mathrm{~g}, 9.83 \mathrm{mmol})$ in THF (18.4 mL) were added iodoform $(0.97 \mathrm{~g}, 2.46 \mathrm{mmol})$, a solution of the resulting aldehyde $(0.61 \mathrm{~g}, 1.23 \mathrm{mmol})$ in THF $(8.0 \mathrm{~mL})$ at $-20{ }^{\circ} \mathrm{C}$ and the mixture was stirred at that temperature for $20 \mathrm{~min}$. After being stirred at $0{ }^{\circ} \mathrm{C}$ for $1.5 \mathrm{~h}$, the reaction mixture was diluted with water $(15 \mathrm{~mL})$ and extracted with $\mathrm{Et}_{2} \mathrm{O}(75 \mathrm{~mL} \times 3)$. The extracts were dried and concentrated. The residue was purified by column chromatography $\left(\mathrm{SiO}_{2} 70 \mathrm{~g}\right.$, hexane/EtOAc $\left.=10: 1\right)$ to afford $7(0.38 \mathrm{~g}, 0.62$ mmol, 50\%) as a pale red oil. $[\alpha]_{\mathrm{D}}^{32}-86.0\left(c 1.00, \mathrm{CHCl}_{3}\right) ;{ }^{1} \mathrm{H} \mathrm{NMR}\left(400 \mathrm{MHz}, \mathrm{CDCl}_{3}\right) \delta 7.60$ (s, 1H), $7.30(\mathrm{~s}, 1 \mathrm{H}), 6.38(\mathrm{dd}, J=14.8,7.2 \mathrm{~Hz}, 1 \mathrm{H}), 6.30(\mathrm{~d}, J=14.8 \mathrm{~Hz}, 1 \mathrm{H}), 4.65$ (d, $J=7.2$ $\mathrm{Hz}, 1 \mathrm{H}), 4.58(\mathrm{~d}, J=7.2 \mathrm{~Hz}, 1 \mathrm{H}), 4.37$ (t, $J=9.8,1 \mathrm{H}), 4.22-4.20(\mathrm{~m}, 1 \mathrm{H}), 4.14(\mathrm{t}, J=11.6 \mathrm{~Hz}$, $1 \mathrm{H}), 4.00(\mathrm{dd}, J=11.6,5.2 \mathrm{~Hz}, 1 \mathrm{H}), 3.81(\mathrm{~s}, 3 \mathrm{H}), 3.65-3.52(\mathrm{~m}, 2 \mathrm{H}), 3.21(\mathrm{dt}, J=10.5,5.2 \mathrm{~Hz}$, 1H), $2.34(\mathrm{~s}, 3 \mathrm{H}), 1.74-1.68(\mathrm{~m}, 1 \mathrm{H}), 1.56(\mathrm{~s}, 3 \mathrm{H}), 1.45(\mathrm{~s}, 3 \mathrm{H}), 0.90(\mathrm{t}, J=8.6 \mathrm{~Hz}, 3 \mathrm{H}), 0.02$ (s, 9H); ${ }^{13} \mathrm{C}$ NMR $\left(100 \mathrm{MHz}, \mathrm{CDCl}_{3}\right) \delta 169.2,166.2,152.6,146.6,130.3,129.1,121.0,98.7$, 93.2, 78.4, 69.1, 65.5, 64.6, 52.1, 45.4, 39.6, 29.4, 19.2, 18.0, 14.4, -1.4; FT-IR (neat) v 2951, 1713, 1637, 1436, 1383, 1250, 941, 838, 764, $692 \mathrm{~cm}^{-1}$; MS (ESI) $m / z: 231,266,301,379,413$, 449, 494, 519 (100), 563, 607, $646\left[(\mathrm{M}+\mathrm{Na})^{+}\right], 725,767$; HRMS (ESI) calcd for $\mathrm{C}_{24} \mathrm{H}_{38} \mathrm{INNaO}_{6} \mathrm{SSi}\left[(\mathrm{M}+\mathrm{Na})^{+}\right]$646.1132, found 646.1117.

\section{Experiments in Scheme 3}

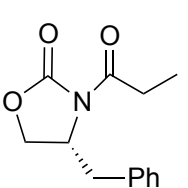

(R)-4-Benzyl-3-propionyloxazolidin-2-one (19): ${ }^{4}$ To a solution of $(R)-4$ benzyl-2-oxazolidinone $(25.1 \mathrm{~g}, 0.142 \mathrm{~mol})$ in THF $(240 \mathrm{~mL})$ was added dropwise $n$-butyllithium $(2.69 \mathrm{M}$ in hexane, $58.7 \mathrm{~mL}, 0.158 \mathrm{~mol})$ at $-78{ }^{\circ} \mathrm{C}$ over $15 \mathrm{~min}$ and the mixture was stirred at $-78{ }^{\circ} \mathrm{C}$ for $10 \mathrm{~min}$. To this mixture was 
added propionyl chloride $(18.5 \mathrm{~mL}, 0.205 \mathrm{~mol})$ and the mixture was stirred at $-78^{\circ} \mathrm{C}$ for $16 \mathrm{~h}$. The reaction was quenched with saturated $\mathrm{NH}_{4} \mathrm{Cl}(200 \mathrm{~mL})$ and the mixture was extracted with $\mathrm{CH}_{2} \mathrm{Cl}_{2}(400 \mathrm{~mL})$. The extracts were washed with brine $(200 \mathrm{~mL})$, dried, and concentrated. The residue was purified by column chromatography $\left(\mathrm{SiO}_{2} 1200 \mathrm{~g}\right.$, hexane/EtOAc $\left.=6: 1\right)$ to give 19 (32.5 g, $0.140 \mathrm{mmol}, 98 \%)$ as a colorless oil. $[\alpha]_{\mathrm{D}}{ }^{24}-66.1\left(c \mathrm{c} 0.87, \mathrm{CHCl}_{3}\right) ;{ }^{1} \mathrm{H} \mathrm{NMR}(400$ $\left.\mathrm{MHz}, \mathrm{CDCl}_{3}\right) \delta$ 7.37-7.22 (m, 5H), 4.72-4.67 (m, 1H), 4.25-4.13 (m, 2H), $3.32(\mathrm{dd}, J=13.2$, $3.2 \mathrm{~Hz}, 1 \mathrm{H}), 3.07-2.90(\mathrm{~m}, 2 \mathrm{H}), 2.79(\mathrm{dd}, J=13.2,9.6 \mathrm{~Hz}, 1 \mathrm{H}), 1.23(\mathrm{t}, J=10.8 \mathrm{~Hz}, 3 \mathrm{H}) ;{ }^{13} \mathrm{C}$ NMR (100 MHz, $\left.\mathrm{CDCl}_{3}\right) \delta 174.1,153.5,135.3,129.4,128.9,127.3,66.2,55.1,37.9,29.2,8.3$; FT-IR (neat) v 3545, 3385, 2943, 1803, 1407, 711, 589, 496, 438, $414 \mathrm{~cm}^{-1}$; HRMS (ESI) calcd for $\mathrm{C}_{13} \mathrm{H}_{15} \mathrm{NNaO}_{3}\left[(\mathrm{M}+\mathrm{Na})^{+}\right]$256.0950, found 256.0926.

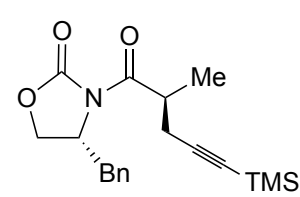

(R)-3-((S)-2-Methyl-5-(trimethylsilyl)pent-4-ynoyl)-4-benzyloxazolidin-2-one (S13): ${ }^{5}$ To a solution of 19 (1.02 g, $\left.4.38 \mathrm{mmol}\right)$ in THF $(25 \mathrm{~mL})$ was added dropwise NaHMDS (1.0 M in THF, $4.5 \mathrm{~mL}, 4.5 \mathrm{mmol})$ at $-78^{\circ} \mathrm{C}$ and the mixture was stirred for $30 \mathrm{~min}$. To this mixture was added a solution of 3-bromo-1-(trimethylsilyl)-1-propyne $(2.1 \mathrm{~mL}, 13.1 \mathrm{mmol})$ in toluene $(5.0 \mathrm{~mL})$, and the mixture was allowed to warm to $0{ }^{\circ} \mathrm{C}$ and stirred at that temperature for $11 \mathrm{~h}$. The reaction was quenched with saturated $\mathrm{NH}_{4} \mathrm{Cl}(20 \mathrm{~mL})$, and the mixture was extracted with EtOAc $(50 \mathrm{~mL})$. The extracts were washed with brine $(20 \mathrm{~mL})$, dried, and concentrated. The residue was purified by column chromatography $\left(\mathrm{SiO}_{2} 40 \mathrm{~g}\right.$, hexane/EtOAc $\left.=6: 1\right)$ to afford $\mathbf{S 1 3}(0.94 \mathrm{~g}, 2.73 \mathrm{mmol}, 62 \%)$ as a colorless oil. $[\alpha]_{\mathrm{D}}{ }^{24}-40.7\left(c 1.02, \mathrm{CHCl}_{3}\right)$ [Lit. of enantiomer +40.7 (c 1.1, $\left.\left.\mathrm{CHCl}_{3}\right)^{6}\right] ;{ }^{1} \mathrm{H}$ NMR $\left(400 \mathrm{MHz}, \mathrm{CDCl}_{3}\right) \delta$ 7.36-7.27 (m, 3H), 7.24-7.22 (m, 2H), $4.71(\mathrm{tt}, J=9.6,3.2 \mathrm{~Hz}, 1 \mathrm{H}), 4.24-4.16(\mathrm{~m}, 2 \mathrm{H}), 3.31$ (dd, $J=13.2,3.2 \mathrm{~Hz}, 1 \mathrm{H}), 2.76$ (dd, $J=$ $13.2,9.6 \mathrm{~Hz}, 1 \mathrm{H}), 3.94$ (q, $J=6.8 \mathrm{~Hz}, 1 \mathrm{H}), 2.60(\mathrm{dt}, J=15.4,6.8 \mathrm{~Hz}, 1 \mathrm{H}), 2.57$ (dt, $J=15.4$, $6.8 \mathrm{~Hz}, 1 \mathrm{H}), 1.27(\mathrm{~d}, J=6.8 \mathrm{~Hz}, 3 \mathrm{H}), 0.13(\mathrm{~s}, 9 \mathrm{H}) ;{ }^{13} \mathrm{C} \mathrm{NMR}\left(100 \mathrm{MHz}, \mathrm{CDCl}_{3}\right) \delta 175.1,160.0$, 135.2 , 129.4, 128.9, 127.3, 103.7, 86.5, 66.1, 60.3, 55.2, 38.0, 37.4, 24.0, 16.5, 14.1, 0.00; FTIR (neat) v 2962, 2175, 1793, 1252, 1026, 860, 760, 643, 574, $503 \mathrm{~cm}^{-1}$; MS (EI) $\mathrm{m} / z: 43$ (100), 73, 91, 117, 166, 167, 216, 226, 250, 298, 328, $343\left(\mathrm{M}^{+}\right)$; HRMS (EI) calcd for $\mathrm{C}_{19} \mathrm{H}_{25} \mathrm{NO}_{3} \mathrm{Si}$ $\left(\mathrm{M}^{+}\right)$343.1604, found 343.1617.

Spectroscopic data in accord with the literature data. ${ }^{5,6}$

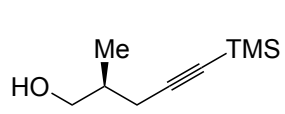

(S)-2-Methyl-5-(trimethylsilyl)pent-4-yn-1-ol (20): To an ice-cooled solution of S13 $(5.67 \mathrm{~g}, 16.5 \mathrm{mmol})$ in THF $(165 \mathrm{~mL})$ and $\mathrm{H}_{2} \mathrm{O}(55 \mathrm{~mL})$ was added sodium borohydride $(2.50 \mathrm{~g}, 66.1 \mathrm{mmol})$, and stirred at room temperature for $2 \mathrm{~h}$. The reaction was quenched with saturated $\mathrm{NH}_{4} \mathrm{Cl}(100 \mathrm{~mL})$ and the mixture was extracted with $\mathrm{Et}_{2} \mathrm{O}(300 \mathrm{~mL})$. The extracts were dried and concentrated. The residue was purified by column chromatography $\left(\mathrm{SiO}_{2} 240 \mathrm{~g}\right.$, hexane/EtOAc $\left.=5: 1\right)$ to afford $20(2.72 \mathrm{~g}$, $16.0 \mathrm{mmol}, 97 \%)$ as a colorless oil. $[\alpha]_{\mathrm{D}}{ }^{26}-7.5\left(c\right.$ 1.02, $\left.\mathrm{CHCl}_{3}\right)$ [Lit. $\left.-6.0\left(c 1.0, \mathrm{CHCl}_{3}\right)^{6}\right]$; ${ }^{1} \mathrm{H}$ 
NMR (400 MHz, $\left.\mathrm{CDCl}_{3}\right) \delta 3.58-3.54(\mathrm{~m}, 2 \mathrm{H}), 2.27$ (dd, $\left.J=6.8,2.4 \mathrm{~Hz}, 2 \mathrm{H}\right), 1.91-1.86(\mathrm{~m}$, 1H), 1.67 (br, $1 \mathrm{H}), 0.99$ (d, $J=6.8 \mathrm{~Hz}, 3 \mathrm{H}), 0.14$ (s, 9H); ${ }^{13} \mathrm{C} \mathrm{NMR}\left(100 \mathrm{MHz}, \mathrm{CDCl}_{3}\right) \delta 105.4$, 86.0, 67.0, 35.0, 23.7, 16.1, 0.00; FT-IR (neat) v 3351, 2960, 1459, 1251, 1038, 855, 761, 699, $651,410 \mathrm{~cm}^{-1}$; MS (EI) m/z: 75, 109, 139 (100), 155, $170\left(\mathrm{M}^{+}\right)$; HRMS (EI) calcd for $\mathrm{C}_{9} \mathrm{H}_{18} \mathrm{OSi}$ $\left(\mathrm{M}^{+}\right)$170.1127, found 170.1122 .

Spectroscopic data in accord with the literature data. 5,6

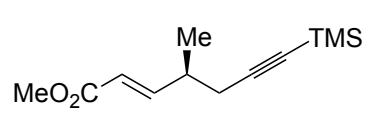

Methyl (S,E)-4-methyl-7-(trimethylsilyl)hept-2-en-6-ynoate (21): To a solution of oxalyl chloride $(253 \mu \mathrm{L}, 2.95 \mathrm{mmol})$ in $\mathrm{CH}_{2} \mathrm{Cl}_{2}(4.0$ $\mathrm{mL})$ was added dropwise a solution of DMSO $(419 \mu \mathrm{L}, 5.90 \mathrm{mmol})$ in $\mathrm{CH}_{2} \mathrm{Cl}_{2}(0.5 \mathrm{~mL})$ at $-78{ }^{\circ} \mathrm{C}$, and the mixture was stirred at $-78^{\circ} \mathrm{C}$ for $30 \mathrm{~min}$. To this mixture was added a solution of $20(251 \mathrm{mg}, 1.48 \mathrm{mmol})$ in $\mathrm{CH}_{2} \mathrm{Cl}_{2}(1.5 \mathrm{~mL})$, and it was stirred at $78^{\circ} \mathrm{C}$ for an additional $1 \mathrm{~h}$. Then triethylamine $(1.7 \mathrm{~mL}, 11.8 \mathrm{mmol})$ was added. After stirring at $0{ }^{\circ} \mathrm{C}$ for $2 \mathrm{~h}$, a solution of (1-carbomethoxyethylidene)triphenylphosphorane $(0.99 \mathrm{~g}, 2.95$ $\mathrm{mmol})$ in $\mathrm{CH}_{2} \mathrm{Cl}_{2}(2.3 \mathrm{~mL})$ was added and stirring was continued at room temperature for $4 \mathrm{~h}$. The mixture was diluted with water $(20 \mathrm{~mL})$ and extracted with EtOAc $(50 \mathrm{~mL})$. The extracts were washed with brine $(20 \mathrm{~mL})$, dried, and concentrated. The residue was purified by column chromatography $\left(\mathrm{SiO}_{2} 12 \mathrm{~g}\right.$, hexane/EtOAc $\left.=4: 1\right)$ to give $21(262.7 \mathrm{mg}, 1.18 \mathrm{mmol}, 79 \%)$ as a pale yellow oil. $[\alpha]_{\mathrm{D}}{ }^{24}+14.4\left(c 0.30, \mathrm{CHCl}_{3}\right) ;{ }^{1} \mathrm{H} \mathrm{NMR}\left(400 \mathrm{MHz}, \mathrm{CDCl}_{3}\right) \delta 6.93(\mathrm{dd}, J=$ 15.6, 7.2 Hz, 1H), 5.84 (d, $J=15.6 \mathrm{~Hz}, 1 \mathrm{H}), 3.74$ (s, 3H), 2.56-2.52 (m, 1H), 2.36-2.25 (m, $2 \mathrm{H}), 1.15(\mathrm{~d}, J=6.8 \mathrm{~Hz}, 3 \mathrm{H}), 0.14(\mathrm{~s}, 9 \mathrm{H}) ;{ }^{13} \mathrm{C} \mathrm{NMR}\left(100 \mathrm{MHz}, \mathrm{CDCl}_{3}\right) \delta 152.2,149.2,120.1$, 104.3, 86.8, 51.4, 47.0, 35.7, 26.4, 18.5, 0.00; FT-IR (neat) $v$ 2960, 2175, 1729, 1658, 1436, 1254, 1033, 852, 761, $647 \mathrm{~cm}^{-1}$; MS (EI) m/z: 73, 89 (100), 209, $224\left(\mathrm{M}^{+}\right)$; HRMS (EI) calcd for $\mathrm{C}_{12} \mathrm{H}_{20} \mathrm{O}_{2} \mathrm{Si}\left(\mathrm{M}^{+}\right)$224.1233, found 224.1232.

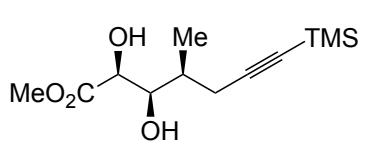

Methyl $(2 R, 3 R, 4 S)-2,3-$ dihydroxy-4-methyl-7-(trimethylsilyl)hept6-ynoate (S14): To an ice-cooled solution of 21 (1.14 g, $5.10 \mathrm{mmol})$ in $t$-butanol $(10 \mathrm{~mL})$ and $\mathrm{H}_{2} \mathrm{O}(10 \mathrm{~mL})$ were added $\mathrm{AD}$-mix- $\beta(7.76 \mathrm{~g})$ and methanesulfonamide $(0.49 \mathrm{~g}, 5.10 \mathrm{mmol})$, and stirred at room temperature for $10.5 \mathrm{~h}$. The mixture was diluted with EtOAc $(50 \mathrm{~mL})$ and saturated $\mathrm{Na}_{2} \mathrm{~S}_{2} \mathrm{O}_{3} \cdot 5 \mathrm{H}_{2} \mathrm{O}(34 \mathrm{~mL})$. After being stirred at room temperature for $45 \mathrm{~min}$, the mixture was filtered through Celite pad. The filtrate was extracted with EtOAc $(100 \mathrm{~mL})$. The extracts were washed with brine $(50 \mathrm{~mL})$, dried, and concentrated. The residue was purified by column chromatography $\left(\mathrm{SiO}_{2} 40 \mathrm{~g}\right.$, hexane/EtOAc $=4: 1)$ to afford S14 (1.25 g, $4.83 \mathrm{mmol}, 95 \%, 9: 1$ diastereomeric mixture) as a colorless oil. For analytic data, the mixture of $\mathbf{S 1 4}$ was partially separated by silica gel chromatography to afford pure compound S14. $[\alpha]_{\mathrm{D}}{ }^{18}+16.8\left(c 1.00, \mathrm{CHCl}_{3}\right) ;{ }^{1} \mathrm{H} \mathrm{NMR}\left(400 \mathrm{MHz}, \mathrm{CDCl}_{3}\right) \delta 4.33$ $(\mathrm{d}, J=2.0 \mathrm{~Hz}, 1 \mathrm{H}), 3.85(\mathrm{dd}, J=7.6,2.0 \mathrm{~Hz}, 1 \mathrm{H}), 3.82(\mathrm{~s}, 3 \mathrm{H}), 2.32(\mathrm{~d}, J=6.0 \mathrm{~Hz}, 2 \mathrm{H}), 1.97-$ $1.92(\mathrm{~m}, 1 \mathrm{H}), 1.14(\mathrm{~d}, J=6.8 \mathrm{~Hz}, 3 \mathrm{H}), 0.14(\mathrm{~s}, 9 \mathrm{H}) ;{ }^{13} \mathrm{C} \mathrm{NMR}\left(100 \mathrm{MHz}, \mathrm{CDCl}_{3}\right) \delta 174.2$, 
104.5, 86.8, 75.1, 71.4, 52.8, 35.3, 24.1, 15.5, 0.00; FT-IR (neat) v 3445, 2957, 2172, 1742 , 1250, 845, 534, 446, 419, $404 \mathrm{~cm}^{-1}$; MS (ESI) $m / z: 178,231,281(100)\left[(\mathrm{M}+\mathrm{Na})^{+}\right]$; HRMS (ESI) calcd for $\mathrm{C}_{12} \mathrm{H}_{22} \mathrm{NaO}_{4} \mathrm{Si}\left[(\mathrm{M}+\mathrm{Na})^{+}\right] 281.1185$, found 281.1214 .

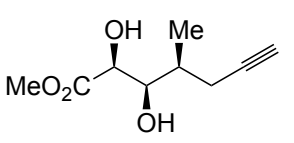

Methyl (2R,3R,4S)-2,3-dihydroxy-4-methylhept-6-ynoate (S15): To an ice-cooled solution of $\mathbf{S 1 4}(105.1 \mathrm{mg}, 407 \mu \mathrm{mol})$ in THF $(4.0 \mathrm{~mL})$ was added tetrabutylammonium fluoride (1.0 M in THF, $0.4 \mathrm{~mL}, 400 \mu \mathrm{mol})$ and the mixture was stirred at room temperature for $30 \mathrm{~min}$. The reaction was quenched with saturated $\mathrm{NH}_{4} \mathrm{Cl}(2 \mathrm{~mL})$, and the mixture was extracted with EtOAc $(20 \mathrm{~mL})$. The extracts were washed with brine $(5 \mathrm{~mL})$, dried, and concentrated. The residue was purified by flash chromatography $\left(\mathrm{SiO}_{2} 5 \mathrm{~g}\right.$, hexane/EtOAc $\left.=5: 1\right)$ to afford $\mathbf{S 1 5}(78.1 \mathrm{mg}, 407 \mu \mathrm{mol}, 100 \%)$ as a colorless oil. $[\alpha]_{\mathrm{D}}{ }^{18}+16.8\left(c 1.00, \mathrm{CHCl}_{3}\right) ;{ }^{1} \mathrm{H} \mathrm{NMR}\left(400 \mathrm{MHz}, \mathrm{CDCl}_{3}\right) \delta 4.32(\mathrm{~d}, J=1.6 \mathrm{~Hz}$, $1 \mathrm{H}), 3.85(\mathrm{t}, J=1.6 \mathrm{~Hz}, 1 \mathrm{H}), 3.83(\mathrm{~s}, 3 \mathrm{H}), 3.43(\mathrm{br}, 1 \mathrm{H}), 2.60(\mathrm{br}, 1 \mathrm{H}), 2.36-2.25(\mathrm{~m}, 2 \mathrm{H}), 2.03$ $(\mathrm{t}, J=2.0 \mathrm{~Hz}, 1 \mathrm{H}), 2.02-1.94(\mathrm{~m}, 1 \mathrm{H}), 1.15(\mathrm{~d}, J=7.2 \mathrm{~Hz}, 3 \mathrm{H}) ;{ }^{13} \mathrm{C}$ NMR $\left(100 \mathrm{MHz}, \mathrm{CDCl}_{3}\right)$ $\delta 174.1,81.8,74.9,71.6,70.1,52.8,35.0,22.6,15.3$; FT-IR (neat) v 3450, 3290, 2956, 1734 , $1439,1221,1119,1041,637 \mathrm{~cm}^{-1}$; MS (EI) $\mathrm{m} / z$ : $186(100)\left(\mathrm{M}^{+}\right)$; HRMS (EI) calcd for $\mathrm{C}_{9} \mathrm{H}_{14} \mathrm{O}_{4}$ $\left(\mathrm{M}^{+}\right)$186.0892, found 186.0893.

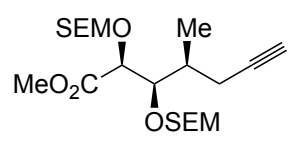

Methyl (2R,3R,4S)-2,3-bis((2-(trimethylsilyl)ethoxy)methoxy)-4-methylhept-6-ynoate (S16): To a solution of $\mathbf{S 1 5}(72.1 \mathrm{mg}, 387 \mu \mathrm{mol})$ in

$\mathrm{CH}_{2} \mathrm{Cl}_{2}(4 \mathrm{~mL})$ were added $N, N$-diisopropylethylamine $(0.5 \mathrm{~mL}, 3.1 \mathrm{mmol})$ and 2-(trimethylsilyl)ethoxymethyl chloride $(0.3 \mathrm{~mL}, 1.6 \mathrm{mmol})$. The mixture was stirred at room temperature for 6 days. The mixture was diluted with $\mathrm{H}_{2} \mathrm{O}(20 \mathrm{~mL})$ and extracted with EtOAc $(100 \mathrm{~mL})$. The extracts were washed with brine $(50 \mathrm{ml})$, dried, and concentrated. The residue was purified by column chromatography $\left(\mathrm{SiO}_{2} 20 \mathrm{~g}\right.$, hexane/EtOAc $\left.=10: 1\right)$ to give S16 (187.9 mg, $387 \mu \mathrm{mol}, 100 \%)$ as a colorless oil. $[\alpha]_{\mathrm{D}}{ }^{21}-17.2\left(c 1.04, \mathrm{CHCl}_{3}\right) ;{ }^{1} \mathrm{H} \mathrm{NMR}(400$ $\left.\mathrm{MHz}, \mathrm{CDCl}_{3}\right) \delta 4.72(\mathrm{~s}, 4 \mathrm{H}), 4.29(\mathrm{~d}, J=4.8 \mathrm{~Hz}, 1 \mathrm{H}), 3.90(\mathrm{t}, J=4.8 \mathrm{~Hz}, 1 \mathrm{H}), 3.74(\mathrm{~s}, 3 \mathrm{H})$, $3.64(\mathrm{t}, J=9.2 \mathrm{~Hz}, 2 \mathrm{H}), 3.61$ (t, $J=7.2 \mathrm{~Hz}, 2 \mathrm{H}), 2.38(\mathrm{dd}, J=16.8,5.2 \mathrm{~Hz}, 1 \mathrm{H}), 2.22$ (dd, $J=$ 16.8, 5.2 Hz, 1H), 1.97 (s, 1H), 1.96-1.94 (m, 1H), 1.09 (d, J=7.2 Hz, 3H), 0.92 (t, $J=7.2 \mathrm{~Hz}$, 2H), 0.90 (t, $J=9.2 \mathrm{~Hz}, 2 \mathrm{H}), 0.00(\mathrm{~s}, 18 \mathrm{H}) ;{ }^{13} \mathrm{C} \mathrm{NMR}\left(100 \mathrm{MHz}, \mathrm{CDCl}_{3}\right) \delta 171.1,96.3,94.7$, $82.5,81.0,76.7,69.6,66.1,65.9,51.9,34.5,22.5,17.9,14.8,-1.6$; FT-IR (neat) v 3306, 2952, 1749, 1430, 1253, 1197, 1110, 1027, 933, $838 \mathrm{~cm}^{-1}$; MS (ESI) m/z: 258, 305, 469 (100) $\left[(\mathrm{M}+\mathrm{Na})^{+}\right]$; HRMS (ESI) calcd for $\mathrm{C}_{21} \mathrm{H}_{42} \mathrm{NaO}_{6} \mathrm{Si}_{2}\left[(\mathrm{M}+\mathrm{Na})^{+}\right]$469.2418, found 469.2398.

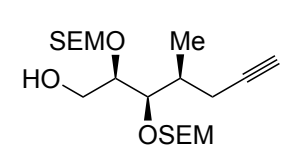

(2R,3R,4S)-2,3-Bis((2-(trimethylsilyl)ethoxy)methoxy)-4-methylhept-6yn-1-ol (12): To a solution of $\mathbf{S 1 6}(750 \mathrm{mg}, 1.68 \mathrm{mmol})$ in $\mathrm{CH}_{2} \mathrm{Cl}_{2}(3.7 \mathrm{~mL})$ was added dropwise diisobutylaluminum hydride (1.02 $\mathrm{M}$ in hexane, 7.4 $\mathrm{mL}, 7.56 \mathrm{mmol}$ ) at $-78^{\circ} \mathrm{C}$ and the mixture was stirred at $-78^{\circ} \mathrm{C}$ for $30 \mathrm{~min}$. The reaction was quenched with saturated Rochelle salt $(20 \mathrm{~mL})$. After being stirred at room temperature for 8 
$\mathrm{h}$, the mixture was extracted with EtOAc $(50 \mathrm{~mL})$. The extracts were washed with brine (20 $\mathrm{mL})$, dried, and concentrated. The residue was purified by column chromatography $\left(\mathrm{SiO}_{2} 20 \mathrm{~g}\right.$, hexane/EtOAc $=2: 1)$ to give $12(671.5 \mathrm{mg}, 1.61 \mathrm{mmol}, 96 \%)$ as a colorless oil. $[\alpha]_{\mathrm{D}}{ }^{19}+6.2(c$ $\left.1.21, \mathrm{CHCl}_{3}\right) ;{ }^{1} \mathrm{H} \mathrm{NMR}\left(400 \mathrm{MHz}, \mathrm{CDCl}_{3}\right) \delta 4.83(\mathrm{~d}, J=7.2 \mathrm{~Hz}, 1 \mathrm{H}), 4.78(\mathrm{~d}, J=7.2 \mathrm{~Hz}, 1 \mathrm{H})$, 4.72 (d, $J=7.2 \mathrm{~Hz}, 1 \mathrm{H}), 4.69$ (d, $J=7.2 \mathrm{~Hz}, 1 \mathrm{H}), 3.80-3.76(\mathrm{~m}, 1 \mathrm{H}), 3.75-3.70(\mathrm{~m}, 4 \mathrm{H}), 3.70-$ $3.65(\mathrm{~m}, 1 \mathrm{H}), 3.65-3.64(\mathrm{~m}, 2 \mathrm{H}), 3.63-3.55(\mathrm{~m}, 1 \mathrm{H}), 2.36(\mathrm{ddd}, J=16.8,5.6,2.8 \mathrm{~Hz}, 1 \mathrm{H}), 2.19$ (ddd, $J=16.8,7.2,2.8 \mathrm{~Hz}, 1 \mathrm{H}), 2.06-2.01(\mathrm{~m}, 1 \mathrm{H}), 1.99$ (t, $J=2.8 \mathrm{~Hz}, 1 \mathrm{H}), 1.07$ (d, $J=6.8$ $\mathrm{Hz}, 3 \mathrm{H}), 1.00-0.93(\mathrm{~m}, 4 \mathrm{H}), 0.01(\mathrm{~s}, 18 \mathrm{H}) ;{ }^{13} \mathrm{C} \mathrm{NMR}\left(100 \mathrm{MHz}, \mathrm{CDCl}_{3}\right) \delta$ 96.6, 95.8, 82.6, $82.1,80.6,69.8,66.1,66.0,62.5,34.1,22.8,18.0,15.1,-1.5$; FT-IR (neat) $v 3462,3308,2954$, $2115,1377,1253,1068,933,862,760,629 \mathrm{~cm}^{-1}$; MS (ESI) $\mathrm{m} / \mathrm{z}: 441$ (100) [(M+Na) $]$; HRMS (ESI) calcd for $\mathrm{C}_{20} \mathrm{H}_{42} \mathrm{NaO}_{5} \mathrm{Si}_{2}\left[(\mathrm{M}+\mathrm{Na})^{+}\right] 441.2469$, found 441.2432.

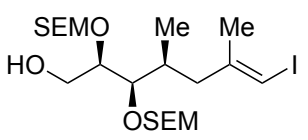

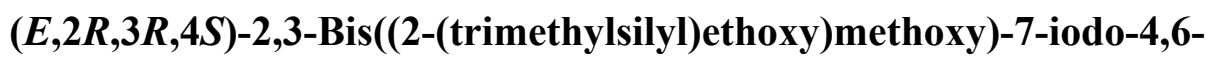
dimethylhept-6-en-1-ol (11): To an ice-cooled solution of tin (II) chloride (5.44 g, $28.7 \mathrm{mmol})$ in THF (24 mL) was added dropwise $n$-butyllithium (2.69 $\mathrm{M}$ in hexane, $33.1 \mathrm{~mL}, 86.0 \mathrm{mmol}$ ). After stirring at $0{ }^{\circ} \mathrm{C}$ for $20 \mathrm{~min}$, methyl magnesium bromide $\left(3.0 \mathrm{M}\right.$ in $\mathrm{Et}_{2} \mathrm{O}, 9.6 \mathrm{~mL}, 28.7 \mathrm{mmol}$ ) was added to the mixture, and stirring was continued at $0{ }^{\circ} \mathrm{C}$ for $20 \mathrm{~min}$. To the mixture were added copper (I) cyanide (128 $\mathrm{mg}, 1.4 \mathrm{mmol}$ ) and a solution of $12(1.00 \mathrm{~g}, 2.4 \mathrm{mmol})$ in THF $(24 \mathrm{~mL})$, and mixture was stirred at $0{ }^{\circ} \mathrm{C}$ for an additional $2 \mathrm{~h}$. Then methyl iodide $(3.0 \mathrm{~mL}, 47.8 \mathrm{mmol})$ was added and the mixture was stirred at $0{ }^{\circ} \mathrm{C}$ for $2 \mathrm{~h}$. Hexane $(50 \mathrm{~mL})$ was added and the mixture was stirred vigorously at $0{ }^{\circ} \mathrm{C}$ for $2 \mathrm{~h}$. After the mixture separated to two layers, the upper layer was added via cannula to an icecooled solution of iodine $(13.3 \mathrm{~g}, 52.6 \mathrm{mmol})$ and sodium carbonate $(3.3 \mathrm{~g}, 31.1 \mathrm{mmol})$ in $\mathrm{CH}_{2} \mathrm{Cl}_{2}(48 \mathrm{~mL})$. The former mixture was washed with hexane $(50 \mathrm{~mL})$ and the upper layer was added to the latter one again. The mixture was stirred at $0{ }^{\circ} \mathrm{C}$ for $12 \mathrm{~h}$, diluted with $\mathrm{Et}_{2} \mathrm{O}$ $(50 \mathrm{~mL})$, and extracted with $\mathrm{Et}_{2} \mathrm{O}(400 \mathrm{~mL})$. The extracts were washed with saturated $\mathrm{Na}_{2} \mathrm{~S}_{2} \mathrm{O}_{3}$ $(150 \mathrm{~mL})$ and brine $(150 \mathrm{~mL})$, dried, and concentrated. The residue was purified by column chromatography $\left(\mathrm{SiO}_{2} 275 \mathrm{~g}\right.$, hexane/EtOAc $\left.=10: 1\right)$ to afford $11(756 \mathrm{mg}, 1.35 \mathrm{mmol}, 56 \%)$ as a colorless oil. $[\alpha]_{\mathrm{D}}{ }^{21}+19.4\left(c 1.00, \mathrm{CHCl}_{3}\right) ;{ }^{1} \mathrm{H} \mathrm{NMR}\left(400 \mathrm{MHz}, \mathrm{CDCl}_{3}\right) \delta 5.94(\mathrm{~s}, 1 \mathrm{H})$, $4.83(\mathrm{~d}, J=6.8 \mathrm{~Hz}, 1 \mathrm{H}), 4.76(\mathrm{~d}, J=6.8 \mathrm{~Hz}, 1 \mathrm{H}), 4.70(\mathrm{~d}, J=6.8 \mathrm{~Hz}, 1 \mathrm{H}), 4.68(\mathrm{~d}, J=6.8 \mathrm{~Hz}$, $1 \mathrm{H}), 3.83-3.76(\mathrm{~m}, 1 \mathrm{H}), 3.72-3.64(\mathrm{~m}, 4 \mathrm{H}), 3.59-3.52(\mathrm{~m}, 2 \mathrm{H}), 3.46(\mathrm{t}, J=4.4 \mathrm{~Hz}, 1 \mathrm{H}), 2.40$ (dd, $J=13.4,5.2 \mathrm{~Hz}, 1 \mathrm{H}), 2.06(\mathrm{dd}, J=13.4,9.2 \mathrm{~Hz}, 1 \mathrm{H}), 1.99-1.94(\mathrm{~m}, 1 \mathrm{H}), 1.80$ (s, 3H), $1.64(\mathrm{t}, J=7.6 \mathrm{~Hz}, 4 \mathrm{H}), 0.87(\mathrm{~d}, J=6.8 \mathrm{~Hz}, 3 \mathrm{H}), 0.02(\mathrm{~s}, 18 \mathrm{H}) ;{ }^{13} \mathrm{C} \mathrm{NMR}\left(100 \mathrm{MHz}, \mathrm{CDCl}_{3}\right)$ $\delta 146.4,144.8,96.7,96.2,83.2,81.5,66.2,66.2,63.0,44.0,32.6,23.8,18.3,18.1,15.5,15.0$, 14.8, -1.3; FT-IR (neat) v 3461, 2953, 1378, 1250, 1192, 1060, 938, 863, 763, $694 \mathrm{~cm}^{-1}$; MS (ESI) $m / z: 583(100)\left[(\mathrm{M}+\mathrm{Na})^{+}\right]$; HRMS (ESI) calcd for $\mathrm{C}_{21} \mathrm{H}_{45} \mathrm{INaO}_{5} \mathrm{Si}_{2}\left[(\mathrm{M}+\mathrm{Na})^{+}\right] 583.1748$, found 583.1776 . 


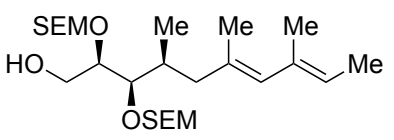

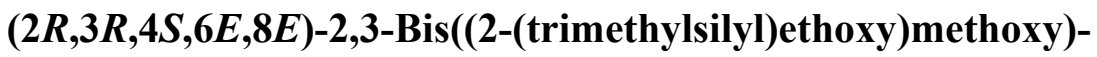

4,6,8-trimethyldeca-6,8-dien-1-ol (23): To a degassed solution of $11(26.4 \mathrm{mg}, 47.1 \mu \mathrm{mol})$ and boronic acid $22(9.4 \mathrm{mg}, 94.3 \mu \mathrm{mol})$ in $\mathrm{CH}_{3} \mathrm{CN}(9.0 \mathrm{~mL})$ and $\mathrm{H}_{2} \mathrm{O}(0.9 \mathrm{~mL})$ were added tetrakis(triphenylphosphine)palladium(0) (5.4 $\mathrm{mg}, 4.7 \mu \mathrm{mol})$ and cesium carbonate $(61.6 \mathrm{mg}, 189 \mu \mathrm{mol})$, and the mixture was stirred at room temperature for $22 \mathrm{~h}$. The reaction was quenched with saturated $\mathrm{NH}_{4} \mathrm{Cl}(5 \mathrm{~mL})$ and the mixture was extracted with EtOAc $(60 \mathrm{~mL})$. The extracts were washed with brine $(30 \mathrm{~mL})$, dried, and concentrated. The residue was purified by preparative TLC (hexane/EtOAc $=2: 1$ ) to give $\mathbf{2 3}$ (19.0 mg, $38.9 \mu \mathrm{mol}, 83 \%)$ as a yellow oil. $[\alpha]_{\mathrm{D}}{ }^{26}+4.8\left(c 0.80, \mathrm{CHCl}_{3}\right) ;{ }^{1} \mathrm{H}$ NMR $(400 \mathrm{MHz}$, $\left.\mathrm{CDCl}_{3}\right) \delta 5.65(\mathrm{~s}, 1 \mathrm{H}), 5.31(\mathrm{q}, J=6.8 \mathrm{~Hz}, 1 \mathrm{H}), 4.84(\mathrm{~d}, J=7.2 \mathrm{~Hz}, 1 \mathrm{H}), 4.78(\mathrm{~d}, J=7.2 \mathrm{~Hz}$, 1H), $4.71(\mathrm{~d}, J=7.2 \mathrm{~Hz}, 1 \mathrm{H}), 4.70(\mathrm{~d}, J=7.2 \mathrm{~Hz}, 1 \mathrm{H}), 3.81(\mathrm{dt}, J=9.6,6.8 \mathrm{~Hz}, 1 \mathrm{H}), 3.68$ (t, $J=8.8 \mathrm{~Hz}, 4 \mathrm{H}), 3.55(\mathrm{dt}, J=9.6,6.8 \mathrm{~Hz}, 2 \mathrm{H}), 3.47(\mathrm{t}, J=4.8 \mathrm{~Hz}, 1 \mathrm{H}), 2.21(\mathrm{dd}, J=13.2,4.8$ $\mathrm{Hz}, 1 \mathrm{H}), 2.00-1.90(\mathrm{~m}, 1 \mathrm{H}), 1.82(\mathrm{dd}, J=13.2,9.6 \mathrm{~Hz}, 1 \mathrm{H}), 1.70(\mathrm{~s}, 6 \mathrm{H}), 1.66(\mathrm{~d}, J=6.8 \mathrm{~Hz}$, $3 \mathrm{H}), 0.96(\mathrm{t}, J=8.8 \mathrm{~Hz}, 4 \mathrm{H}), 0.88(\mathrm{~d}, J=6.8 \mathrm{~Hz}, 3 \mathrm{H}), 0.02(\mathrm{~s}, 18 \mathrm{H}) ;{ }^{13} \mathrm{C}$ NMR $(100 \mathrm{MHz}$, $\left.\mathrm{CDCl}_{3}\right) \delta 133.5,133.0,131.2,123.3,96.6,96.2,83.3,81.8,66.0,66.0,63.1,45.1,32.6,18.1$, 18.0, 17.6, 16.7, 14.7, 13.6, -1.5; FT-IR (neat) v 3466, 2953, 1378, 1250, 1026, 937, 836, 694, $442,422 \mathrm{~cm}^{-1}$; MS (ESI) $\mathrm{m} / z$ : $511(100)\left[(\mathrm{M}+\mathrm{Na})^{+}\right], 527$; HRMS (ESI) calcd for $\mathrm{C}_{25} \mathrm{H}_{52} \mathrm{NaO}_{5} \mathrm{Si}_{2}$ $\left[(\mathrm{M}+\mathrm{Na})^{+}\right]$511.3251, found 511.3239.

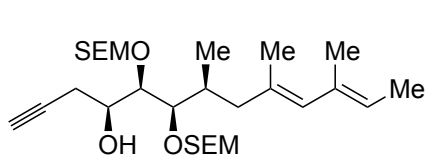

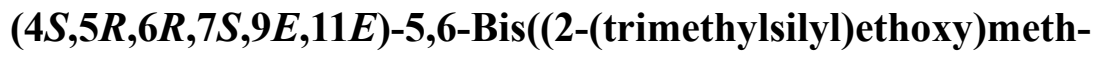
oxy)-7,9,11-trimethyl-trideca-9,11-dien-1-yn-4-ol (8): To a solution of $23(281 \mathrm{mg}, 576 \mu \mathrm{mol})$ in $\mathrm{CH}_{2} \mathrm{Cl}_{2}(115 \mathrm{~mL})$ were

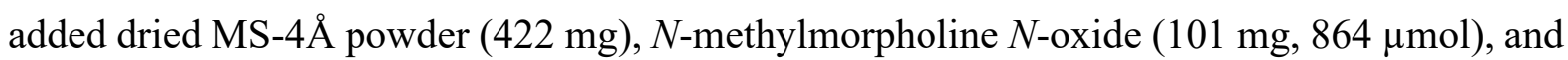
tetrapropylammonium perruthenate $(20.3 \mathrm{mg}, 57.6 \mu \mathrm{mol})$. The mixture was stirred at room temperature for $1 \mathrm{~h}$ and concentrated. The residue was purified by column chromatography $\left(\mathrm{SiO}_{2} 20 \mathrm{~g}\right.$, hexane/EtOAc = 10:1) to afford aldehyde 24 (239 mg, $\left.492 \mu \mathrm{mol}, 85 \%\right)$.

To a solution of $(1 S, 2 R)-(+)$-2-amino-1,2-diphenylethanol $25(525 \mathrm{mg}, 2.46 \mathrm{mmol})$ and indium powder $(283 \mathrm{mg}, 2.46 \mathrm{mmol})$ in THF $(10 \mathrm{~mL})$ backfilled with $\mathrm{Ar}(5 \times)$ were added pyridine $(199 \mu \mathrm{L}, 2.46 \mathrm{mmol})$ and propargyl bromide $(212 \mu \mathrm{L}, 2.46 \mathrm{mmol})$ in that order, and the mixture was stirred at room temperature for $25 \mathrm{~min}$ vigorously. The mixture was cooled to $-78^{\circ} \mathrm{C}$, and a solution of resulting aldehyde $\mathbf{2 4}(239 \mathrm{mg}, 492 \mu \mathrm{mol})$ in THF $(2 \mathrm{~mL})$ was added. The mixture was gradually allowed to warm at room temperature over $24 \mathrm{~h}$ with stirring. The reaction was quenched with saturated $\mathrm{NH}_{4} \mathrm{Cl}(10 \mathrm{~mL})$, and the mixture was extracted with EtOAc $(40 \mathrm{~mL})$. The extracts were washed with brine $(15 \mathrm{~mL})$, dried, and concentrated. The rdesidue was purified by flash chromatography $\left(\mathrm{SiO}_{2} 20 \mathrm{~g}\right.$, hexane/EtOAc $\left.=10: 1\right)$ to afford $8(236 \mathrm{mg}, 449$ $\mu \mathrm{mol}, 91 \%)$ as a colorless oil. $[\alpha]_{\mathrm{D}}{ }^{28}+5.0\left(c \mathrm{c} .79, \mathrm{CHCl}_{3}\right) ;{ }^{1} \mathrm{H} \mathrm{NMR}\left(400 \mathrm{MHz}, \mathrm{CDCl}_{3}\right) \delta 5.65$ $(\mathrm{s}, 1 \mathrm{H}), 5.30(\mathrm{q}, J=6.8 \mathrm{~Hz}, 1 \mathrm{H}), 4.85(\mathrm{~d}, J=6.8 \mathrm{~Hz}, 1 \mathrm{H}), 4.79(\mathrm{~d}, J=6.8 \mathrm{~Hz}, 1 \mathrm{H}), 4.75(\mathrm{~d}, J$ $=6.8 \mathrm{~Hz}, 1 \mathrm{H}), 4.71(\mathrm{~d}, J=6.8 \mathrm{~Hz}, 1 \mathrm{H}), 3.81(\mathrm{t}, J=10.8 \mathrm{~Hz}, 1 \mathrm{H}), 3.80(\mathrm{t}, J=10.8 \mathrm{~Hz}, 1 \mathrm{H})$ 
$3.70(\mathrm{t}, J=7.6 \mathrm{~Hz}, 2 \mathrm{H}), 3.64(\mathrm{q}, J=6.0 \mathrm{~Hz}, 1 \mathrm{H}), 3.58(\mathrm{t}, J=7.6 \mathrm{~Hz}, 1 \mathrm{H}), 3.57(\mathrm{t}, J=7.6 \mathrm{~Hz}$, $1 \mathrm{H}), 3.18(\mathrm{~d}, J=6.0 \mathrm{~Hz}, 1 \mathrm{H}), 2.54(\mathrm{dd}, J=6.0,2.4 \mathrm{~Hz}, 2 \mathrm{H}), 2.24(\mathrm{dd}, J=13.2,4.4 \mathrm{~Hz}, 1 \mathrm{H})$, $2.01(\mathrm{t}, J=2.4 \mathrm{~Hz}, 1 \mathrm{H}), 2.00-1.89(\mathrm{~m}, 1 \mathrm{H}), 1.86(\mathrm{dd}, J=13.2,9.2 \mathrm{~Hz}, 1 \mathrm{H}), 1.70(\mathrm{~s}, 3 \mathrm{H}), 1.69$ $(\mathrm{s}, 3 \mathrm{H}), 1.65(\mathrm{~d}, J=6.8 \mathrm{~Hz}, 3 \mathrm{H}), 0.93$ (t, $J=7.6 \mathrm{~Hz}, 4 \mathrm{H}), 0.87$ (d, $J=6.8 \mathrm{~Hz}, 3 \mathrm{H}), 0.00$ (s, $18 \mathrm{H}) ;{ }^{13} \mathrm{C}$ NMR $\left(100 \mathrm{MHz}, \mathrm{CHCl}_{3}\right) \delta 133.5,133.0,131.1,123.3,97.0,96.3,82.2,81.4,80.8$, $70.4,69.4,66.5,65.9,45.2,32.6,23.9,18.1,18.0,17.6,16.7,14.2,13.6,-1.5,-1.5$; FT-IR (neat) $v 3442,3305,2949,1598,1379,1250,1026,932,848,636 \mathrm{~cm}^{-1}$; MS (ESI) $\mathrm{m} / z: 323,351,373$ (100), 413, 427, 470, 499, 519, $549\left[(\mathrm{M}+\mathrm{Na})^{+}\right], 585,602$; HRMS (ESI) calcd for $\mathrm{C}_{28} \mathrm{H}_{54} \mathrm{NaO}_{5} \mathrm{Si}_{2}\left[(\mathrm{M}+\mathrm{Na})^{+}\right]$549.3408, found 549.3388.

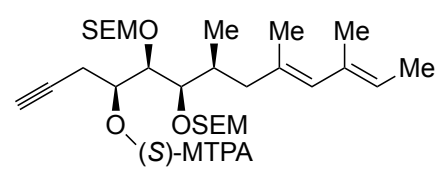

(S)-MTPA ester of 8: ${ }^{1} \mathrm{H}$ NMR $\left(500 \mathrm{MHz}, \mathrm{CDCl}_{3}\right) \delta 7.57(\mathrm{dd}, J=6.0,2.5 \mathrm{~Hz}, 2 \mathrm{H}), 7.41-7.39$ $(\mathrm{m}, 3 \mathrm{H}), 5.65(\mathrm{~s}, 1 \mathrm{H}), 5.34(\mathrm{q}, J=8.0 \mathrm{~Hz}, 1 \mathrm{H}), 4.74-4.69(\mathrm{~m}, 4 \mathrm{H}), 3.92$ (dd, $J=6.5,4.5 \mathrm{~Hz}$, $1 \mathrm{H}), 3.74-3.69(\mathrm{~m}, 1 \mathrm{H}), 3.65-3.58(\mathrm{~m}, 4 \mathrm{H}), 3.51(\mathrm{~s}, 3 \mathrm{H}), 3.45(\mathrm{dd}, J=6.5,4.5 \mathrm{~Hz}, 1 \mathrm{H}), 2.74$ $(\mathrm{dd}, \mathrm{J}=6.0,3.0 \mathrm{~Hz}, 1 \mathrm{H}), 2.65(\mathrm{dd}, J=6.0,3.0 \mathrm{~Hz}, 1 \mathrm{H}), 2.20$ (dd, $J=13.0,4.5 \mathrm{~Hz}, 1 \mathrm{H}), 1.96$ (s, 1H), 1.95-1.94 (m, 1H), $1.88(\mathrm{dd}, J=13.0,9.5 \mathrm{~Hz}, 1 \mathrm{H}), 1.70(\mathrm{~s}, 3 \mathrm{H}), 1.69$ (s, 3H), 1.66 (d, $J=8.0 \mathrm{~Hz}, 3 \mathrm{H}), 0.95-0.89(\mathrm{~m}, 4 \mathrm{H}), 0.85$ (d, $J=6.8 \mathrm{~Hz}, 3 \mathrm{H}), 0.02$ (s, 9H), -0.01 (s, 9H).

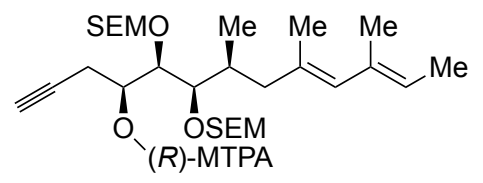

(R)-MTPA ester of 8: ${ }^{1} \mathrm{H}$ NMR $\left(500 \mathrm{MHz}, \mathrm{CDCl}_{3}\right) \delta 7.60(\mathrm{dd}, \mathrm{J}=6.0,2.5 \mathrm{~Hz}, 2 \mathrm{H}), 7.40-7.37$ $(\mathrm{m}, 3 \mathrm{H}), 5.63(\mathrm{~s}, 1 \mathrm{H}), 5.32(\mathrm{q}, J=8.0 \mathrm{~Hz}, 1 \mathrm{H}), 4.69-4.63(\mathrm{~m}, 2 \mathrm{H}), 4.50(\mathrm{~d}, J=7.5 \mathrm{~Hz}, 1 \mathrm{H})$, $4.31(\mathrm{~d}, J=7.5 \mathrm{~Hz}, 1 \mathrm{H}), 3.86(\mathrm{t}, J=5.5 \mathrm{~Hz}, 1 \mathrm{H}), 3.69-3.61(\mathrm{~m}, 2 \mathrm{H}), 3.65(\mathrm{~s}, 3 \mathrm{H}), 3.62-3.60$ $(\mathrm{m}, 1 \mathrm{H}), 3.58(\mathrm{dd}, J=9.6,1.5 \mathrm{~Hz}, 1 \mathrm{H}), 3.51(\mathrm{dd}, J=9.6,1.5 \mathrm{~Hz}, 1 \mathrm{H}), 3.34$ (t, $J=5.5 \mathrm{~Hz}, 1 \mathrm{H})$, $2.79(\mathrm{dd}, J=5.0,2.5 \mathrm{~Hz}, 1 \mathrm{H}), 2.76(\mathrm{dd}, J=5.0,2.5 \mathrm{~Hz}, 1 \mathrm{H}), 2.20(\mathrm{dd}, J=13.0,4.5 \mathrm{~Hz}, 1 \mathrm{H})$, $2.03(\mathrm{~s}, 1 \mathrm{H}), 1.96-1.92(\mathrm{~m}, 1 \mathrm{H}), 1.82(\mathrm{dd}, J=13.0,10.0 \mathrm{~Hz}, 1 \mathrm{H}), 1.72(\mathrm{~s}, 3 \mathrm{H}), 1.71(\mathrm{~s}, 3 \mathrm{H})$, $1.67(\mathrm{~d}, J=8.0 \mathrm{~Hz}, 3 \mathrm{H}), 0.91-0.87(\mathrm{~m}, 4 \mathrm{H}), 0.79$ (d, $J=6.8 \mathrm{~Hz}, 3 \mathrm{H}), 0.02$ (s, 9H), 0.00 (s, 9H).

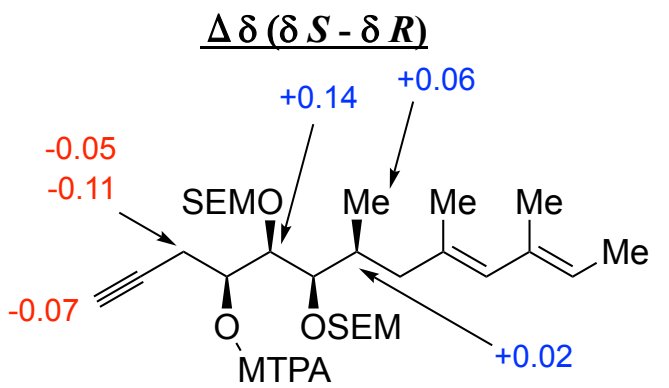




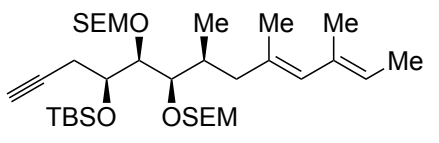

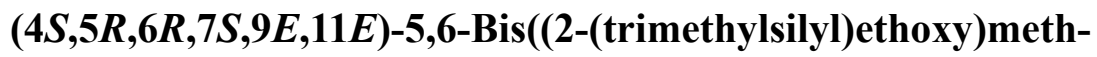
oxy)-4-(tert-butyl)dimethylsiloxy-7,9,11-trimethyltrideca-

9,11-dien-1-yne (26): To an ice-cooled solution of 8 (11.4 mg, was stirred at $0{ }^{\circ} \mathrm{C}$ for $15 \mathrm{~min}$. To the mixture was added TBSOTf $(26.7 \mu \mathrm{L}, 116 \mu \mathrm{mol})$ and the mixture was stirred at room temperature for $16 \mathrm{~h}$. The mixture was quenched with saturated $\mathrm{NH}_{4} \mathrm{Cl}(500 \mu \mathrm{L})$ at $0{ }^{\circ} \mathrm{C}$, and extracted with EtOAc $(10 \mathrm{~mL})$. The extracts were washed with brine $(10 \mathrm{~mL})$, dried, and concentrated. The residue was purified by flash chromatography $\left(\mathrm{SiO}_{2} 5 \mathrm{~g}\right.$, hexane/EtOAc $\left.=40: 1\right)$ to give compound $26(15.1 \mathrm{mg}, 21.7 \mu \mathrm{mol}, 100 \%)$ as a colorless oil. $[\alpha]_{\mathrm{D}}{ }^{25}+26.7\left(c 1.00, \mathrm{CHCl}_{3}\right) ;{ }^{1} \mathrm{H}$ NMR $\left(400 \mathrm{MHz}, \mathrm{CDCl}_{3}\right) \delta 5.67(\mathrm{~s}, 1 \mathrm{H}), 5.30(\mathrm{q}$, $J=6.8 \mathrm{~Hz}, 1 \mathrm{H}), 4.79(\mathrm{~d}, J=6.8 \mathrm{~Hz}, 1 \mathrm{H}), 4.68(\mathrm{~d}, J=6.8 \mathrm{~Hz}, 1 \mathrm{H}), 4.76(\mathrm{~d}, J=6.8 \mathrm{~Hz}, 1 \mathrm{H})$, $4.72(\mathrm{~d}, J=6.8 \mathrm{~Hz}, 1 \mathrm{H}), 3.78(\mathrm{t}, J=9.4 \mathrm{~Hz}, 1 \mathrm{H}), 3.72(\mathrm{t}, J=9.4 \mathrm{~Hz}, 1 \mathrm{H}) 3.64(\mathrm{t}, J=6.4 \mathrm{~Hz}$, $1 \mathrm{H}), 3.57$ (t, $J=7.2 \mathrm{~Hz}, 2 \mathrm{H}), 3.52$ (t, $J=7.2 \mathrm{~Hz}, 2 \mathrm{H}), 2.59$ (dd, $J=12.8,2.4 \mathrm{~Hz}, 1 \mathrm{H}), 2.35-2.23$ (m, 2H), 2.12-2.11 (m, 1H), $1.93(\mathrm{t}, J=2.4 \mathrm{~Hz}, 1 \mathrm{H}), 1.87(\mathrm{dd}, J=12.8,9.2 \mathrm{~Hz}, 1 \mathrm{H}), 1.72(\mathrm{~s}$, $3 \mathrm{H}), 1.69$ (s, 3H), 1.65 (d, $J=6.8 \mathrm{~Hz}, 3 \mathrm{H}), 0.94$ (t, $J=6.8 \mathrm{~Hz}, 4 \mathrm{H}), 0.90$ (s, 9H), 0.84 (d, $J=$ $6.8 \mathrm{~Hz}, 3 \mathrm{H}), 0.15$ (s, 3H), 0.13 (s, 3H), $0.01(\mathrm{~s}, 18 \mathrm{H}) ;{ }^{13} \mathrm{C} \mathrm{NMR}\left(100 \mathrm{MHz}, \mathrm{CHCl}_{3}\right) \delta 133.6$, $131.2,126.4,123.3,96.9,96.7,82.5,81.0,80.8,72.5,69.9,65.8,45.4,32.4,26.0,23.0,18.3$, 18.1, 16.8, 14.1, 14.0, 13.6, -1.4, -4.2, -4.4; FT-IR (neat) v 3313, 2953, 2858, 1462, 1376, 1250, 1105, 1023, 836, $455 \mathrm{~cm}^{-1}$; MS (ESI) $\mathrm{m} / \mathrm{z}$ : 164, 215, 241, 288, 349, 380, 413, 486, 531, 575, $663(100)\left[(\mathrm{M}+\mathrm{Na})^{+}\right]$; HRMS (ESI) calcd for $\mathrm{C}_{34} \mathrm{H}_{68} \mathrm{NaO}_{5} \mathrm{Si}_{3}\left[(\mathrm{M}+\mathrm{Na})^{+}\right]$663.4272, found 663.4299 .

\section{Experiments in Scheme 4}

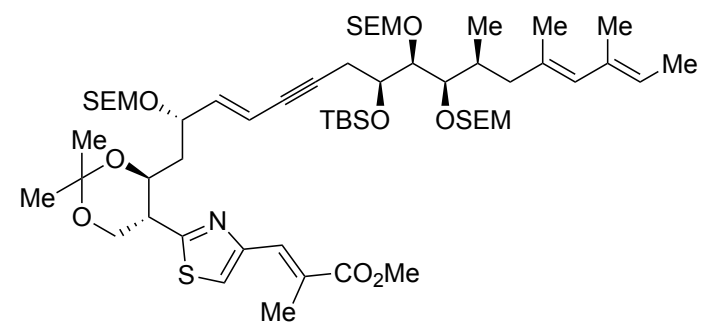

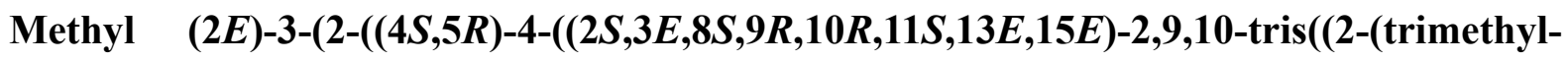
silyl)ethoxy)methoxy)-8-tert-butyldimethylsiloxy-11,13,15-trimethylheptadeca-3,13,15trien-5-ynyl)-2,2-dimethyl-1,3-dioxan-5-yl)thiazol-4-yl)-2-meth-ylacrylate (27): To a degassed solution of vinyl iodide 7 (383 mg, $0.615 \mathrm{mmol}$ ) and alkyne 26 (394 mg, $0.615 \mathrm{mmol}$ ) in $\mathrm{CH}_{3} \mathrm{CN}$ (31 mL) were added copper iodide (58.6 mg, $\left.0.308 \mathrm{mmol}\right)$ and bis(triphenylphosphine)palladium(II) dichloride $(43.2 \mathrm{mg}, 61.5 \mu \mathrm{mol})$ at $-20{ }^{\circ} \mathrm{C}$, and the mixture was stirred at $-20^{\circ} \mathrm{C}$ for $5 \mathrm{~min}$. To this mixture was added triethylamine $(515 \mu \mathrm{L}, 3.69$ $\mathrm{mmol}$ ), and stirring was continued at $-20^{\circ} \mathrm{C}$ for $15 \mathrm{~min}$. After being stirred at room temperature 
for $1.5 \mathrm{~h}$, the mixture was neutralized by phosphate buffer $\left(\mathrm{KH}_{2} \mathrm{PO}_{4} / \mathrm{Na}_{2} \mathrm{HPO}_{4}, \mathrm{pH} 7.0,10 \mathrm{~mL}\right)$ and extracted with EtOAc $(240 \mathrm{~mL})$. The extracts were dried and concentrated. The residue was purified by flash chromatography $\left(\mathrm{SiO}_{2} 100 \mathrm{~g}\right.$, hexane/EtOAc $\left.=10: 1\right)$ to afford $27(626$ $\mathrm{mg}, 0.55 \mathrm{mmol}, 90 \%)$ as a yellow oil. $[\alpha]_{\mathrm{D}}{ }^{23}-47.5\left(c 1.00, \mathrm{CHCl}_{3}\right) ;{ }^{1} \mathrm{H} \mathrm{NMR}\left(300 \mathrm{MHz}, \mathrm{CDCl}_{3}\right)$ $\delta 7.61(\mathrm{~s}, 1 \mathrm{H}), 7.31(\mathrm{~s}, 1 \mathrm{H}), 5.79(\mathrm{dd}, J=16.2,7.2 \mathrm{~Hz}, 1 \mathrm{H}), 5.67(\mathrm{~s}, 1 \mathrm{H}), 5.60(\mathrm{~d}, J=16.2 \mathrm{~Hz}$, $1 \mathrm{H}), 5.30(\mathrm{q}, J=6.8 \mathrm{~Hz}, 1 \mathrm{H}), 4.78(\mathrm{~d}, J=6.9 \mathrm{~Hz}, 1 \mathrm{H}), 4.74(\mathrm{~d}, J=6.9 \mathrm{~Hz}, 2 \mathrm{H}), 4.72(\mathrm{~d}, J=$ $6.9 \mathrm{~Hz}, 1 \mathrm{H}), 4.62(\mathrm{~d}, J=6.9 \mathrm{~Hz}, 1 \mathrm{H}), 4.56(\mathrm{~d}, J=6.9 \mathrm{~Hz}, 1 \mathrm{H}), 4.40(\mathrm{t}, J=9.6 \mathrm{~Hz}, 1 \mathrm{H}), 4.25$ $(\mathrm{t}, J=7.8 \mathrm{~Hz}, 1 \mathrm{H}), 4.12(\mathrm{dd}, J=13.0,7.2 \mathrm{~Hz}, 1 \mathrm{H}), 4.00(\mathrm{dd}, J=13.0,4.8 \mathrm{~Hz}, 1 \mathrm{H}), 3.81(\mathrm{~s}$, $3 \mathrm{H}), 3.74(\mathrm{dd}, J=7.8,1.8 \mathrm{~Hz}, 1 \mathrm{H}), 3.69$ (t, $J=7.8 \mathrm{~Hz}, 1 \mathrm{H}), 3.55-3.52$ (m, 7H), 3.20 (dt, $J=$ 10.4, $4.8 \mathrm{~Hz}, 1 \mathrm{H}), 2.68(\mathrm{~d}, J=7.8 \mathrm{~Hz}, 1 \mathrm{H}), 2.42$ (d, $J=7.8 \mathrm{~Hz}, 1 \mathrm{H}), 2.35$ (s, 3H), 2.24 (dd, $J$ $=12.8,4.5 \mathrm{~Hz}, 1 \mathrm{H}), 2.05-2.03(\mathrm{~m}, 1 \mathrm{H}), 1.87(\mathrm{dd}, J=12.8,8.8 \mathrm{~Hz}, 1 \mathrm{H}), 1.72(\mathrm{~s}, 3 \mathrm{H}), 1.70(\mathrm{~s}$, $3 \mathrm{H}), 1.66(\mathrm{~d}, J=6.8 \mathrm{~Hz}, 3 \mathrm{H}), 1.58(\mathrm{~s}, 3 \mathrm{H}), 1.56-1.50(\mathrm{~m}, 2 \mathrm{H}), 1.46(\mathrm{~s}, 3 \mathrm{H}), 0.97-0.89(\mathrm{~m}, 6 \mathrm{H})$, 0.89 (s, 9H), 0.84 (d, $J=6.0 \mathrm{~Hz}, 3 \mathrm{H}), 0.12$ (s, 3H), 0.10 (s, 3H), 0.02 (s, 27H); ${ }^{13} \mathrm{C}$ NMR (100 $\left.\mathrm{MHz}, \mathrm{CDCl}_{3}\right) \delta 169.4,166.5,152.8,142.1,135.4,133.8,133.6,131.1,130.6,129.3,127.8$, $123.3,121.2,112.3,98.9,96.8,96.7,93.0,89.5,80.9,79.7,72.7,72.4,69.4,65.8,65.6,64.7$, $60.5,52.2,45.5,45.4,40.3,32.3,29.5,25.9,23.8,19.3,18.3,18.1,18.0,17.9,16.8,14.4,14.1$, 13.7, -1.4, -4.3, -4.5; FT-IR (neat) v 2952, 1714, 1436, 1382, 1249, 1108, 1025, 838, 693, 456 $\mathrm{cm}^{-1}$; MS (ESI) $\mathrm{m} / \mathrm{z}: 178,301,375,413,520,561,740,839,942,1073,1159$ (100) $\left[(\mathrm{M}+\mathrm{Na})^{+}\right]$; HRMS (ESI) calcd for $\mathrm{C}_{58} \mathrm{H}_{105} \mathrm{NNaO}_{11} \mathrm{SSi}_{4}\left[(\mathrm{M}+\mathrm{Na})^{+}\right] 1158.6383$, found 1158.6396.

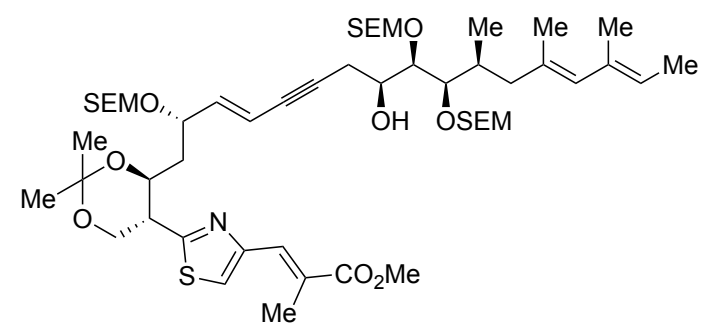

Methyl (2E)-3-(2-((4S,5R)-4-((2S,3E,8S,9R,10R,11S,13E,15E)-2,9,10-tris((2-(trimethylsilyl) ethoxy)methoxy)-8-hydroxy-11,13,15-trimethylheptadeca-3,13,15-trien-5-ynyl)-2,2dimethyl-1,3-dioxan-5-yl)thiazol-4-yl)-2-methylacrylate (6): To an ice-cooled solution of 27 $(626 \mathrm{mg}, 0.55 \mathrm{mmol})$ in THF $(5.5 \mathrm{~mL})$ was added tetrabutylammonium fluoride (1.0 M in THF, $1.7 \mathrm{~mL}, 1.7 \mathrm{mmol}$ ) and the mixture was stirred at room temperature for $2.5 \mathrm{~h}$. Tetrabutylammonium fluoride (1.0 M in THF, $1.2 \mathrm{~mL}, 1.2 \mathrm{mmol}$ ) was added again, and the mixture was stirred at room temperature for $12 \mathrm{~h}$. The reaction was quenched with saturated $\mathrm{NH}_{4} \mathrm{Cl}(5 \mathrm{~mL})$, and the mixture was extracted with EtOAc $(20 \mathrm{~mL})$. The extracts were washed with brine $(5 \mathrm{~mL})$, dried, and concentrated. The residue was purified by column chromatography $\left(\mathrm{SiO}_{2} 100 \mathrm{~g}\right.$, hexane/EtOAc $\left.=10: 1\right)$ to afford $6(524 \mathrm{mg}, 0.51 \mathrm{mmol}, 93 \%)$ as a yellow oil. $[\alpha]_{\mathrm{D}}{ }^{21}-24.7\left(c 0.50, \mathrm{CHCl}_{3}\right) ;{ }^{1} \mathrm{H} \mathrm{NMR}\left(500 \mathrm{MHz}, \mathrm{CDCl}_{3}\right) \delta 7.60(\mathrm{~s}, 1 \mathrm{H}), 7.30(\mathrm{~s}$, $1 \mathrm{H}), 5.84(\mathrm{dd}, J=16.0,7.2 \mathrm{~Hz}, 1 \mathrm{H}), 5.65(\mathrm{~s}, 1 \mathrm{H}), 5.62$ (d, $J=16.0 \mathrm{~Hz}, 1 \mathrm{H}), 5.31$ (q, $J=7.0$ 
$\mathrm{Hz}, 1 \mathrm{H}), 4.85(\mathrm{~d}, J=7.0 \mathrm{~Hz}, 1 \mathrm{H}), 4.78(\mathrm{~d}, J=7.0 \mathrm{~Hz}, 1 \mathrm{H}), 4.76(\mathrm{~d}, J=7.0 \mathrm{~Hz}, 1 \mathrm{H}), 4.72(\mathrm{~d}, J$ $=7.0 \mathrm{~Hz}, 1 \mathrm{H}), 4.61(\mathrm{~d}, J=7.0 \mathrm{~Hz}, 1 \mathrm{H}), 4.55(\mathrm{~d}, J=7.0 \mathrm{~Hz}, 1 \mathrm{H}), 4.39(\mathrm{t}, J=10.5 \mathrm{~Hz}, 1 \mathrm{H}), 4.24$ (t, $J=10.5 \mathrm{~Hz}, 1 \mathrm{H}), 4.12(\mathrm{dd}, J=11.5,7.0 \mathrm{~Hz}, 1 \mathrm{H}), 4.00(\mathrm{dd}, J=11.5,5.5 \mathrm{~Hz}, 1 \mathrm{H}), 3.81$ (s, $3 \mathrm{H}), 3.79(\mathrm{t}, J=6.0 \mathrm{~Hz}, 1 \mathrm{H}), 3.70-3.66(\mathrm{~m}, 2 \mathrm{H}), 3.63$ (dt, $J=8.0,2.0 \mathrm{~Hz}, 2 \mathrm{H}), 3.60-3.54(\mathrm{~m}$, $4 \mathrm{H}), 3.19(\mathrm{dt}, J=10.5,5.5 \mathrm{~Hz}, 1 \mathrm{H}), 2.69-2.62(\mathrm{~m}, 2 \mathrm{H}), 2.35(\mathrm{~s}, 3 \mathrm{H}), 2.24$ (dd, $J=13.0,4.5 \mathrm{~Hz}$, $1 \mathrm{H}), 1.95-1.92(\mathrm{~m}, 1 \mathrm{H}), 1.86(\mathrm{dd}, J=13.0,9.0 \mathrm{~Hz}, 1 \mathrm{H}), 1.71(\mathrm{~s}, 3 \mathrm{H}), 1.70(\mathrm{~s}, 3 \mathrm{H}), 1.66(\mathrm{~d}, J=$ $7.0 \mathrm{~Hz}, 3 \mathrm{H}), 1.58(\mathrm{~s}, 3 \mathrm{H}), 1.56-1.45(\mathrm{~m}, 2 \mathrm{H}), 1.46$ (s, 3H), 1.00-0.89 (m, 6H), 0.86 (d, $J=6.5$ $\mathrm{Hz}, 3 \mathrm{H}), 0.01(\mathrm{~s}, 27 \mathrm{H}) ;{ }^{13} \mathrm{C} \mathrm{NMR}\left(100 \mathrm{MHz}, \mathrm{CDCl}_{3}\right) \delta 169.4,166.4,152.7,142.8,133.2,131.3$, $130.5,129.2,123.4,121.2,111.8,98.9,97.1,96.4,93.1,87.2,82.5,81.6,72.4,69.8,69.4,66.6$, $66.0,65.7,64.7,52.3,45.6,45.3,40.4,32.7,29.6,25.1,19.4,18.2,18.1,17.8,16.9,14.5,14.3$, 13.8, 0.1, -1.2, -1.3; FT-IR (neat) v 3456, 2952, 2360, 1712, 1381, 1248, 1108, 1022, 836, 423 $\mathrm{cm}^{-1}$; MS (ESI) m/z: 186, 242 (100), 331, 360, 413, 441, 536, 608, 699, 771, 841, 939, 1045 $\left[(\mathrm{M}+\mathrm{Na})^{+}\right]$; HRMS (ESI) calcd for $\mathrm{C}_{52} \mathrm{H}_{91} \mathrm{NNaO}_{11} \mathrm{SSi}_{3}\left[(\mathrm{M}+\mathrm{Na})^{+}\right] 1044.5518$, found 1044.5531 .

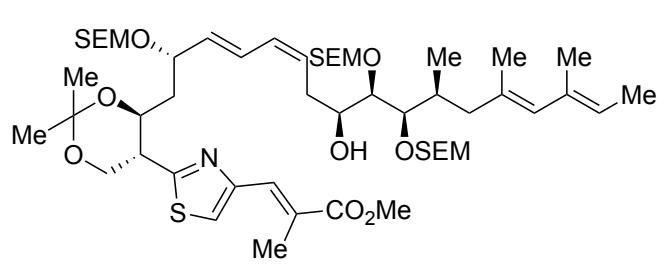

Methyl (2E)-3-(2-((4S,5R)-4-((2S,3E,5Z,8S,9R,10R,11S,13E,15E)-2,9,10-tris((2-(trimethylsilyl)ethoxy)methoxy)-8-hydroxy-11,13,15-trimethylheptadeca-3,5,13,15-tetraenyl)2,2-dimethyl-1,3-dioxan-5-yl)thiazol-4-yl)-2-methylacrylate (29): To an ice-cooled 6 (524 $\mathrm{mg}, 513 \mu \mathrm{mol}$ ) was added 1,1,3,3-tetramethyldisilazane (684 mg, $889 \mu \mathrm{mol})$, and the mixture was stirred at $0{ }^{\circ} \mathrm{C}$ for $5 \mathrm{~min}$. After being stirred at room temperature for $20 \mathrm{~h}$, the mixture was concentrated. To an ice-cooled solution of the resulting mixture in THF $(10 \mathrm{~mL})$ was added platinum(0)-1,3-divinyl-1,1,3,3-tetramethyldisiloxane complex solution in xylenes $(\sim 2 \% \mathrm{Pt}$, $171 \mu \mathrm{L}, 383 \mu \mathrm{mol})$, and mixture was stirred at room temperature for $1 \mathrm{~h}$ and concentrated. Then, to a solution of the resulting mixture 28 in THF $(6.4 \mathrm{~mL})$ was added the solution of silver(I) fluoride $(651 \mathrm{mg}, 5.1 \mathrm{mmol})$ in water $(1.2 \mathrm{~mL})$, methanol $(3.7 \mathrm{~mL})$, and DMSO $(1.8$ $\mathrm{mL})$. After being stirred at room temperature for $1 \mathrm{~h}$, the mixture was concentrated. The residue was purified by flash chromatography $\left(\mathrm{SiO}_{2} 85 \mathrm{~g}\right.$, hexane/EtOAc $\left.=8: 1\right)$ to afford $29(313 \mathrm{mg}$, $306 \mu \mathrm{mol}, 60 \%)$ as a colorless oil. $[\alpha]_{\mathrm{D}}{ }^{28}-24.6\left(c 0.67, \mathrm{CHCl}_{3}\right) ;{ }^{1} \mathrm{H} \mathrm{NMR}\left(400 \mathrm{MHz}, \mathrm{CDCl}_{3}\right)$ $\delta 7.59(\mathrm{~s}, 1 \mathrm{H}), 7.29(\mathrm{~s}, 1 \mathrm{H}), 6.46(\mathrm{dd}, J=15.2,11.2 \mathrm{~Hz}, 1 \mathrm{H}), 6.04(\mathrm{t}, J=11.2 \mathrm{~Hz}, 1 \mathrm{H}), 5.64(\mathrm{~s}$, $1 \mathrm{H}), 5.54$ (dt, $J=11.2,7.6 \mathrm{~Hz}, 1 \mathrm{H}), 5.45$ (dd, $J=15.2,7.2 \mathrm{~Hz}, 1 \mathrm{H}), 5.30$ (q, $J=7.0 \mathrm{~Hz}, 1 \mathrm{H})$, $4.83(\mathrm{~d}, J=6.8 \mathrm{~Hz}, 1 \mathrm{H}), 4.78(\mathrm{~d}, J=6.8 \mathrm{~Hz}, 1 \mathrm{H}), 4.75(\mathrm{~d}, J=6.8 \mathrm{~Hz}, 1 \mathrm{H}), 4.70(\mathrm{~d}, J=6.8 \mathrm{~Hz}$, $1 \mathrm{H}), 4.63(\mathrm{~d}, J=6.8 \mathrm{~Hz}, 1 \mathrm{H}), 4.55(\mathrm{~d}, J=6.8 \mathrm{~Hz}, 1 \mathrm{H}), 4.41$ (t, $J=11.2 \mathrm{~Hz}, 1 \mathrm{H}), 4.29$ (t, $J=$ $7.2 \mathrm{~Hz}, 1 \mathrm{H}), 4.13$ (dd, $J=12.0,11.2 \mathrm{~Hz}, 1 \mathrm{H}), 3.99$ (dd, $J=12.0,5.2 \mathrm{~Hz}, 1 \mathrm{H}), 3.83$ (dt, $J=6.0$, 
$5.6 \mathrm{~Hz}, 1 \mathrm{H}), 3.80(\mathrm{~s}, 3 \mathrm{H}), 3.68(\mathrm{t}, J=6.0 \mathrm{~Hz}, 1 \mathrm{H}), 3.66(\mathrm{t}, J=9.2 \mathrm{~Hz}, 2 \mathrm{H}), 3.61(\mathrm{t}, J=8.8 \mathrm{~Hz}$, $1 \mathrm{H}), 3.57(\mathrm{t}, J=6.0 \mathrm{~Hz}, 1 \mathrm{H}), 3.54(\mathrm{dt}, J=7.6,4.0 \mathrm{~Hz}, 2 \mathrm{H}), 3.49(\mathrm{dd}, J=9.2,3.6 \mathrm{~Hz}, 1 \mathrm{H}), 3.19$ $(\mathrm{dt}, J=11.2,5.2 \mathrm{~Hz}, 1 \mathrm{H}), 2.91(\mathrm{~d}, J=5.6 \mathrm{~Hz}, 1 \mathrm{H}), 2.52(\mathrm{dt}, J=9.6,7.6 \mathrm{~Hz}, 1 \mathrm{H}), 2.41(\mathrm{dt}, J=$ 9.6, 7.6 Hz, 1H), 2.33 (s, 3H), 2.21 (dd, $J=12.8,4.0 \mathrm{~Hz}, 1 \mathrm{H}), 1.93-1.85$ (m, 1H), 1.81 (dd, $J$ $=12.8,9.6 \mathrm{~Hz}, 1 \mathrm{H}), 1.69(\mathrm{~s}, 3 \mathrm{H}), 1.69(\mathrm{~s}, 3 \mathrm{H}), 1.65(\mathrm{~d}, J=7.0 \mathrm{~Hz}, 3 \mathrm{H}), 1.57(\mathrm{~s}, 3 \mathrm{H}), 1.56-1.45$ $(\mathrm{m}, 2 \mathrm{H}), 1.45(\mathrm{~s}, 3 \mathrm{H}), 0.95(\mathrm{t}, J=7.6 \mathrm{~Hz}, 2 \mathrm{H}), 0.93(\mathrm{t}, J=8.8 \mathrm{~Hz}, 2 \mathrm{H}), 0.90(\mathrm{t}, J=9.2 \mathrm{~Hz}, 2 \mathrm{H})$, $0.83(\mathrm{~d}, J=6.8 \mathrm{~Hz}, 3 \mathrm{H}), 0.00(\mathrm{~s}, 27 \mathrm{H}) ;{ }^{13} \mathrm{C} \mathrm{NMR}\left(100 \mathrm{MHz}, \mathrm{CDCl}_{3}\right) \delta 169.2,166.4,161.7$, 152.6, 135.1, 131.4, 130.4, 130.0, 129.0, 127.9, 127.2, 126.4, 121.3, 121.0, 98.7, 97.0, 96.3, $92.8,82.9,81.5,72.7,70.8,69.3,66.4,65.8,65.3,64.6,52.1,45.5,40.6,32.4,29.7,29.5,23.9$, 19.3, 18.1, 18.0, 17.5, 15.0, 14.4, 14.3, 13.6, -1.4, -1.4; FT-IR (neat) v 3491, 2951, 1712, 1440 , 1379, 1251, 1106, 1026, 840, $763 \mathrm{~cm}^{-1}$; MS (FAB) $m / z:$ 59, 73 (100), 109, 136, 210, 266, 332, $418,476,570,628,709,877,1047\left[(\mathrm{M}+\mathrm{Na})^{+}\right]$; HRMS (FAB) calcd for $\mathrm{C}_{52} \mathrm{H}_{93} \mathrm{NNaO}_{11} \mathrm{SSi}_{3}$ $\left[(\mathrm{M}+\mathrm{Na})^{+}\right] 1046.5675$, found 1046.5679 .

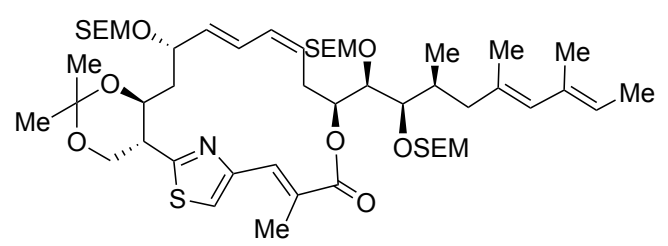

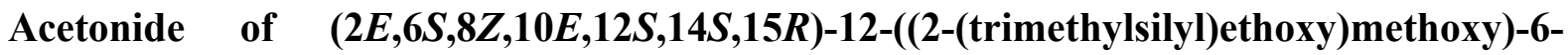

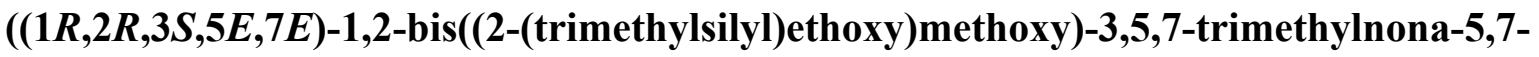
dienyl)-14-hydroxy-15-(hydroxymethyl)-3-methyl-5-oxa-17-thia-19-aza-bicyclo[14.2.1]nonadeca-1(18),2,8,10-tetraen-4-one (30): To an ice-cooled solution of 29 (94.4 mg, 92.2 $\mu \mathrm{mol})$ in methanol $(18 \mathrm{~mL})$ was added $3 \mathrm{M} \mathrm{KOH}(9 \mathrm{~mL})$. The mixture was stirred at $35^{\circ} \mathrm{C}$ for $11 \mathrm{~h}$. The mixture was neutralized with $3 \mathrm{M} \mathrm{HCl}(9 \mathrm{~mL})$ and extracted with EtOAc $(200 \mathrm{~mL})$. The extracts were washed with brine $(150 \mathrm{~mL})$, dried, and concentrated to give the corresponding crude carboxylic acid $(93.1 \mathrm{mg}, 92.2 \mu \mathrm{mol})$.

To a solution of the resulting carboxylic acid $(93.1 \mathrm{mg}, 92.2 \mu \mathrm{mol})$ in toluene $(461 \mathrm{~mL})$ were

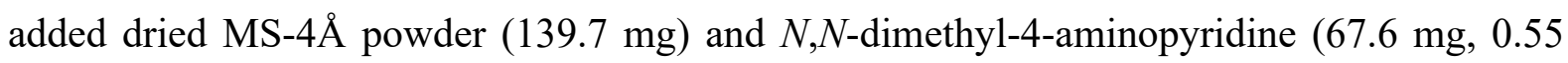
$\mathrm{mmol}$ ), and the mixture was stirred at $70{ }^{\circ} \mathrm{C}$ for $5 \mathrm{~min}$. To the mixture was added 2-methyl-6nitrobenzoic anhydride ( $95.3 \mathrm{mg}, 0.28 \mathrm{mmol}$ ), and then stirring was continued at $70{ }^{\circ} \mathrm{C}$ for 14 h. The mixture was filtered through Celite pad, and concentrated. The residue was purified by flash chromatography $\left(\mathrm{SiO}_{2} 25 \mathrm{~g}\right.$, hexane/EtOAc $\left.=5: 1\right)$ to afford $30(66.6 \mathrm{mg}, 67.2 \mu \mathrm{mol}, 73 \%)$ as a colorless oil. $[\alpha]_{\mathrm{D}}{ }^{28}-6.7\left(c 0.33, \mathrm{CHCl}_{3}\right) ;{ }^{1} \mathrm{H} \mathrm{NMR}\left(400 \mathrm{MHz}, \mathrm{CDCl}_{3}\right) \delta 7.77(\mathrm{~s}, 1 \mathrm{H}), 7.29$ $(\mathrm{s}, 1 \mathrm{H}), 6.55(\mathrm{dd}, J=14.8,11.6 \mathrm{~Hz}, 1 \mathrm{H}), 6.04(\mathrm{t}, J=11.6 \mathrm{~Hz}, 1 \mathrm{H}), 5.66(\mathrm{~s}, 1 \mathrm{H}), 5.64(\mathrm{dd}, J=$ $14.8,9.2 \mathrm{~Hz}, 1 \mathrm{H}), 5.32(\mathrm{dd}, J=11.6,4.8 \mathrm{~Hz}, 1 \mathrm{H}), 5.26(\mathrm{q}, J=7.2 \mathrm{~Hz}, 1 \mathrm{H}), 5.10(\mathrm{dd}, J=12.4$, $3.6 \mathrm{~Hz}, 1 \mathrm{H}), 4.85(\mathrm{~d}, J=6.8 \mathrm{~Hz}, 1 \mathrm{H}), 4.80(\mathrm{~d}, J=6.8 \mathrm{~Hz}, 2 \mathrm{H}), 4.73(\mathrm{~d}, J=6.8 \mathrm{~Hz}, 1 \mathrm{H}), 4.68$ $(\mathrm{d}, J=6.8 \mathrm{~Hz}, 1 \mathrm{H}), 4.64(\mathrm{~d}, J=6.8 \mathrm{~Hz}, 1 \mathrm{H}), 4.63-4.58(\mathrm{~m}, 1 \mathrm{H}), 4.29(\mathrm{dt}, J=9.2,5.6 \mathrm{~Hz}, 1 \mathrm{H})$, $4.16(\mathrm{dd}, J=10.8,5.6 \mathrm{~Hz}, 1 \mathrm{H}), 4.01$ (t, $J=10.8 \mathrm{~Hz}, 1 \mathrm{H}), 3.85$ (dd, $J=6.4,3.6 \mathrm{~Hz}, 1 \mathrm{H}), 3.78$ 
$(\mathrm{t}, J=6.4 \mathrm{~Hz}, 1 \mathrm{H}), 3.60(\mathrm{t}, J=7.6 \mathrm{~Hz}, 2 \mathrm{H}), 3.57(\mathrm{t}, J=7.6 \mathrm{~Hz}, 2 \mathrm{H}), 3.54(\mathrm{t}, J=7.6 \mathrm{~Hz}, 2 \mathrm{H})$, $3.41(\mathrm{dt}, J=10.8,5.6 \mathrm{~Hz}, 1 \mathrm{H}), 2.95(\mathrm{dt}, J=12.4,11.6 \mathrm{~Hz}, 1 \mathrm{H}), 2.35-2.28(\mathrm{~m}, 2 \mathrm{H}), 2.33(\mathrm{t}, J=$ $4.8 \mathrm{~Hz}, 1 \mathrm{H}), 2.26(\mathrm{dd}, J=12.8,4.0 \mathrm{~Hz}, 1 \mathrm{H}), 2.15-2.08(\mathrm{~m}, 1 \mathrm{H}), 2.05(\mathrm{~s}, 3 \mathrm{H}), 1.91$ (dd, $J=12.8$, $9.6 \mathrm{~Hz}, 1 \mathrm{H}), 1.77$ (s, 3H), 1.67 (s, 3H), $1.62(\mathrm{~d}, J=7.2 \mathrm{~Hz}, 3 \mathrm{H}), 1.50(\mathrm{~s}, 3 \mathrm{H}), 1.44$ (s, 3H), 0.94 $(\mathrm{t}, J=7.6 \mathrm{~Hz}, 6 \mathrm{H}), 0.81(\mathrm{~d}, J=6.4 \mathrm{~Hz}, 3 \mathrm{H}), 0.00(\mathrm{~s}, 27 \mathrm{H}) ;{ }^{13} \mathrm{C} \mathrm{NMR}\left(100 \mathrm{MHz}, \mathrm{CDCl}_{3}\right) \delta$ $167.9,165.1,150.8,135.3,133.6,133.3,133.2,131.7,130.9,127.9,127.3,126.5,123.0,118.1$, 99.0, 96.6, 96.2, 91.7, 81.4, 79.2, 73.6, 70.9, 65.9, 65.7, 65.0, 63.6, 45.9, 43.2, 38.3, 32.0, 30.3, 29.3, 19.0, 18.1, 18.0, 17.8, 16.7, 13.8, 13.6, 13.3, -1.3, -1.4, -1.4; FT-IR (neat) v 2925, 2857, $1711,1455,1376,1254,1106,1027,840,480 \mathrm{~cm}^{-1}$; MS (ESI) $\mathrm{m} / z: 219,301,382,519,572$ (100), 573, 613, 657, 858, 958, $1014\left[(\mathrm{M}+\mathrm{Na})^{+}\right]$; HRMS (ESI) calcd for $\mathrm{C}_{51} \mathrm{H}_{89} \mathrm{NNaO}_{10} \mathrm{SSi}_{3}$ $\left[(\mathrm{M}+\mathrm{Na})^{+}\right]$1014.5413, found 1014.5438.

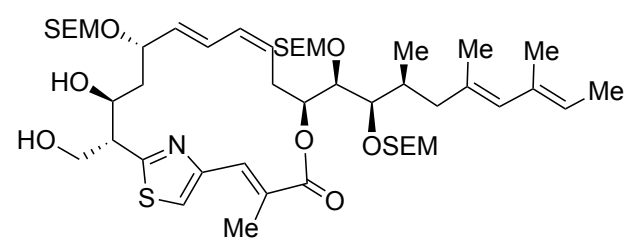

$(2 E, 6 S, 8 Z, 10 E, 12 S, 14 S, 15 R)-12-((2-(T r i m e t h y l s i l y l) e t h o x y) m e t h o x y)-6-((1 R, 2 R, 3 S, 5 E$, 7E)-1,2-bis((2-(trimethylsilyl)ethoxy)methoxy)-3,5,7-trimethylnona-5,7-dienyl)-14hydroxy-15-(hydroxymethyl)-3-methyl-5-oxa-17-thia-19-aza-bicyclo[14.2.1]-nonadeca1(18),2,8,10-tetraen-4-one (31): To an ice-cooled solution of $\mathbf{3 0}(5.3 \mathrm{mg}, 5.4 \mu \mathrm{mol})$ in THF $(1.0 \mathrm{~mL})$ was added $3 \mathrm{M} \mathrm{HClO}_{4}(0.1 \mathrm{~mL})$ and the mixture was stirred at room temperature for $4 \mathrm{~h}$. The reaction was quenched with saturated $\mathrm{NaHCO}_{3}(2.0 \mathrm{~mL})$ and the mixture was extracted with EtOAc $(20 \mathrm{~mL})$. The extracts were washed with brine $(5 \mathrm{~mL})$, dried, and concentrated. The residue was purified by flash chromatography $\left(\mathrm{SiO}_{2} 1 \mathrm{~g}\right.$, hexane/EtOAc $\left.=4: 1\right)$ to afford $31(5.1 \mathrm{mg}, 5.4 \mu \mathrm{mol}, 100 \%)$ as a colorless oil. $[\alpha]_{\mathrm{D}}{ }^{19}-86.5\left(c 0.56, \mathrm{CHCl}_{3}\right) ;{ }^{1} \mathrm{H} \mathrm{NMR}(400$ $\left.\mathrm{MHz}, \mathrm{CDCl}_{3}\right) \delta 7.85(\mathrm{~s}, 1 \mathrm{H}), 7.32(\mathrm{~s}, 1 \mathrm{H}), 6.63(\mathrm{dd}, J=15.2,11.6 \mathrm{~Hz}, 1 \mathrm{H}), 5.99(\mathrm{t}, J=11.6$ $\mathrm{Hz}, 1 \mathrm{H}), 5.66(\mathrm{~s}, 1 \mathrm{H}), 5.49$ (dd, $J=15.2,8.8 \mathrm{~Hz}, 1 \mathrm{H}), 5.36$ (dt, $J=11.6,4.8 \mathrm{~Hz}, 1 \mathrm{H}), 5.26$ (q, $J=6.4 \mathrm{~Hz}, 1 \mathrm{H}), 5.09$ (dd, $J=10.8,3.2 \mathrm{~Hz}, 1 \mathrm{H}), 5.02(\mathrm{t}, J=8.4 \mathrm{~Hz}, 1 \mathrm{H}), 4.84(\mathrm{~d}, J=6.8 \mathrm{~Hz}$, $1 \mathrm{H}), 4.82(\mathrm{~d}, J=6.8 \mathrm{~Hz}, 1 \mathrm{H}), 4.80(\mathrm{~d}, J=6.8 \mathrm{~Hz}, 1 \mathrm{H}), 4.73(\mathrm{~d}, J=6.8 \mathrm{~Hz}, 1 \mathrm{H}), 4.71$ (d, $J=$ $6.8 \mathrm{~Hz}, 1 \mathrm{H}), 4.59$ (d, $J=6.8 \mathrm{~Hz}, 1 \mathrm{H}), 4.23-4.15(\mathrm{~m}, 2 \mathrm{H}), 4.15$ (t, $J=5.2 \mathrm{~Hz}, 1 \mathrm{H}), 3.85$ (dd, $J$ $=8.0,3.2 \mathrm{~Hz}, 1 \mathrm{H}), 3.81(\mathrm{dd}, J=10.8,8.0 \mathrm{~Hz}, 1 \mathrm{H}), 3.77(\mathrm{dd}, J=8.4,2.4 \mathrm{~Hz}, 1 \mathrm{H}), 3.74(\mathrm{t}, J=$ $8.4 \mathrm{~Hz}, 1 \mathrm{H}), 3.62(\mathrm{t}, J=6.8 \mathrm{~Hz}, 1 \mathrm{H}), 3.61(\mathrm{t}, J=6.8 \mathrm{~Hz}, 1 \mathrm{H}), 3.54(\mathrm{t}, J=10.8 \mathrm{~Hz}, 2 \mathrm{H}), 3.53$ $(\mathrm{t}, J=7.2 \mathrm{~Hz}, 2 \mathrm{H}), 3.13-3.05(\mathrm{~m}, 1 \mathrm{H}), 2.91(\mathrm{dt}, J=14.0,10.8 \mathrm{~Hz}, 1 \mathrm{H}), 2.36(\mathrm{dd}, J=14.0,4.8$ $\mathrm{Hz}, 1 \mathrm{H}), 2.25$ (dd, $J=12.0,4.4 \mathrm{~Hz}, 1 \mathrm{H}), 2.22$ (t, $J=8.0 \mathrm{~Hz}, 1 \mathrm{H}), 2.12-2.04(\mathrm{~m}, 1 \mathrm{H}), 2.03$ (s, $3 \mathrm{H}), 1.91(\mathrm{dd}, J=12.0,9.6 \mathrm{~Hz}, 1 \mathrm{H}), 1.80(\mathrm{~s}, 3 \mathrm{H}), 1.67$ (s, 3H), $1.62(\mathrm{~d}, J=6.4 \mathrm{~Hz}, 3 \mathrm{H}), 1.12$ $(\mathrm{dd}, J=8.0,5.2 \mathrm{~Hz}, 1 \mathrm{H}), 0.96(\mathrm{t}, J=6.8 \mathrm{~Hz}, 2 \mathrm{H}), 0.94(\mathrm{t}, J=10.8 \mathrm{~Hz}, 2 \mathrm{H}), 0.93$ (t, $J=7.2 \mathrm{~Hz}$, $2 \mathrm{H}), 0.80(\mathrm{~d}, J=6.8 \mathrm{~Hz}, 3 \mathrm{H}), 0.00(\mathrm{~s}, 27 \mathrm{H}) ;{ }^{13} \mathrm{C} \mathrm{NMR}\left(100 \mathrm{MHz}, \mathrm{CDCl}_{3}\right) \delta 167.5,166.4,150.4$, 133.6, 133.6, 133.4, 133.3, 131.2, 130.9, 128.3, 127.3, 123.0, 118.9, 96.6, 96.2, 92.0, 81.4, 79.1, 
75.6, 73.4, 73.3, 65.9, 65.9, 65.7, 50.3, 45.9, 32.0, 30.2, 18.2, 18.1, 18.0, 17.8, 16.7, 13.7, 13.6, 13.3, -1.4, -1.4; FT-IR (neat) v 3485, 2952, 1709, 1421, 1245, 1105, 1024, 934, 840, $759 \mathrm{~cm}^{-1}$; MS (FAB) $m / z:$ 59, 73 (100), 109, 136, 147, 178, 226, 268, 356, 556, 628, 805, 878, 936, 975 $\left[(\mathrm{M}+\mathrm{Na})^{+}\right]$; HRMS (FAB) calcd for $\mathrm{C}_{48} \mathrm{H}_{85} \mathrm{NNaO}_{10} \mathrm{SSi}_{3}\left[(\mathrm{M}+\mathrm{Na})^{+}\right]$974.5100, found 974.5099.

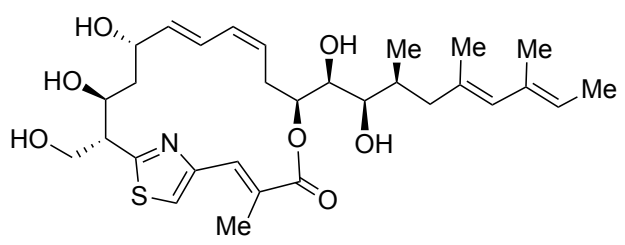

Thuggacin cmc-A (1): To an ice-cooled solution of $\mathbf{3 1}(6.0 \mathrm{mg}, 6.31 \mu \mathrm{mol})$ in methanol (500 $\mu \mathrm{L})$ was added methanolic hydrogen chloride $(500 \mu \mathrm{L})$ [prepared by treating anhydrous methanol $(2.5 \mathrm{~mL})$ with acetyl chloride $(100 \mu \mathrm{L})]$, and the mixture was stirred at room temperature for $15 \mathrm{~min}$. The mixture was diluted with triethylamine $(145 \mu \mathrm{L})$ and concentrated. The residue was purified by flash chromatography $\left(\mathrm{SiO}_{2} 1 \mathrm{~g}\right.$, hexane/EtOAc $\left.=1: 1\right)$, and reverse phase preparative TLC $\left(\mathrm{MeOH} / \mathrm{H}_{2} \mathrm{O}=4: 1\right)$ to afford thuggacin cmc-A (1) $(1.6 \mathrm{mg}, 2.85 \mu \mathrm{mol}$, $45 \%)$ as a white solid. $[\alpha]_{\mathrm{D}}{ }^{22}-148.5(c 0.04, \mathrm{MeOH})\left[\right.$ Lit. $\left.[\alpha]_{\mathrm{D}}{ }^{22}-160.6(c 1.24, \mathrm{MeOH})^{7}\right]$; ${ }^{1} \mathrm{H}$ NMR (500 MHz, DMSO-d 6 ) $\delta 7.87(\mathrm{~s}, 1 \mathrm{H}), 7.83(\mathrm{~s}, 1 \mathrm{H}), 6.45(\mathrm{dd}, J=15.0,11.0 \mathrm{~Hz}, 1 \mathrm{H}), 5.87$ $(\mathrm{dd}, J=11.0,11.0 \mathrm{~Hz}, 1 \mathrm{H}), 5.58(\mathrm{~s}, 1 \mathrm{H}), 5.38(\mathrm{dd}, J=15.0,9.2 \mathrm{~Hz}, 1 \mathrm{H}), 5.30(\mathrm{ddd}, J=11.3$, $11.3,5.4 \mathrm{~Hz}, 1 \mathrm{H}), 5.24(\mathrm{q}, J=6.8 \mathrm{~Hz}, 1 \mathrm{H}), 5.17$ (t, $J=4.6 \mathrm{~Hz}, 1 \mathrm{H}), 5.07$ (d, $J=3.0 \mathrm{~Hz}, 1 \mathrm{H})$, $4.98(\mathrm{~d}, J=6.5 \mathrm{~Hz}, 1 \mathrm{H}), 4.94(\mathrm{dd}, J=9.6,9.2 \mathrm{~Hz}, 1 \mathrm{H}), 4.90(\mathrm{dd}, J=11.2,3.3 \mathrm{~Hz}, 1 \mathrm{H}), 4.84$ $(\mathrm{d}, J=6.0 \mathrm{~Hz}, 1 \mathrm{H}), 4.32(\mathrm{~d}, J=5.8 \mathrm{~Hz}, 1 \mathrm{H}), 3.94(\mathrm{~m}, 2 \mathrm{H}), 3.89-3.85(\mathrm{~m}, 1 \mathrm{H}), 3.58(\mathrm{dd}, J=$ 6.4, 3.3 Hz, 1H), 3.44-3.41 (m, 1H), 3.30 (m, 1H), 2.85 (ddd, $J=13.7,11.9,11.3 \mathrm{~Hz}, 1 \mathrm{H}), 2.23$ (dd, $J=13.7,5.0 \mathrm{~Hz}, 1 \mathrm{H}), 2.11(\mathrm{dd}, J=12.8,6.4 \mathrm{~Hz}, 1 \mathrm{H}), 1.95(\mathrm{~s}, 3 \mathrm{H}), 1.94-1.93(\mathrm{~m}, 1 \mathrm{H})$, $1.76(\mathrm{dd}, J=12.8,8.0 \mathrm{~Hz}, 1 \mathrm{H}), 1.70(\mathrm{~s}, 3 \mathrm{H}), 1.66-1.65(\mathrm{~m}, 1 \mathrm{H}), 1.63(\mathrm{~s}, 3 \mathrm{H}), 1.58(\mathrm{~d}, J=6.8$ $\mathrm{Hz}, 3 \mathrm{H}), 0.75(\mathrm{~d}, J=6.7 \mathrm{~Hz}, 3 \mathrm{H}), 0.41(\mathrm{dd}, J=14.5,1.5 \mathrm{~Hz}, 1 \mathrm{H}) ;{ }^{13} \mathrm{C}$ NMR (125 MHz, DMSO$\left.\mathrm{d}_{6}\right) \delta 168.0,166.9,149.0,137.2,133.5,133.3,133.3,131.0,130.2,128.1,126.0,125.0,122.7$, 121.1, 74.2, 72.1, 71.8, 70.1, 69.5, 59.8, 51.4, 45.3, 39.2, 32.3, 30.0, 17.7, 16.7, 13.7, 13.5, 13.4; FT-IR (neat) v 3401, 2923, 2853, 1707, 1460, 1377, 1284, 1258, 1101, $1042 \mathrm{~cm}^{-1}$; MS (ESI) $m / z: 193,254,329,413,519,544,584(100)\left[(\mathrm{M}+\mathrm{Na})^{+}\right]$; HRMS (ESI) calcd for $\mathrm{C}_{30} \mathrm{H}_{43} \mathrm{NNaO}_{7} \mathrm{~S}\left[(\mathrm{M}+\mathrm{Na})^{+}\right]$584.2658, found 584.2661. 
NMR Comparison of Synthetic Thuggacin cmc-A with the Natural Product

Table S1. Comparison of ${ }^{1} \mathrm{H}-\mathrm{NMR}$ Spectral Data between Natural and Synthetic Thuggacin cmc-A (1)

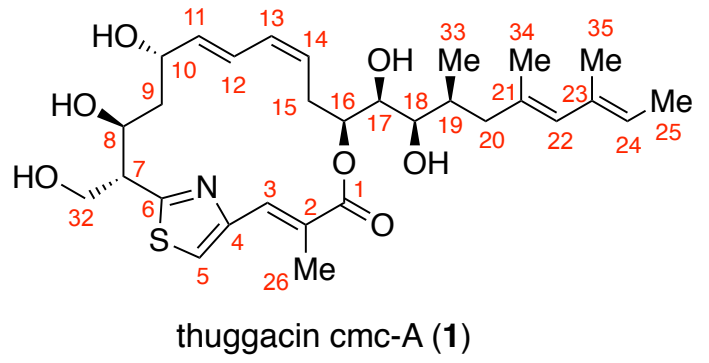

\begin{tabular}{|c|c|c|c|}
\hline & $\begin{array}{c}\text { Natural Thuggacin cmc-A (1) } \\
{ }^{1} \mathrm{H}(600 \mathrm{MHz})^{7}\end{array}$ & $\begin{array}{c}\text { Synthetic Thuggacin cmc-A (1) } \\
{ }^{1} \mathrm{H}(500 \mathrm{MHz})\end{array}$ & $\begin{array}{l}\Delta \delta \text { (synthetic 1- } \\
\text { natural) ppm }\end{array}$ \\
\hline Position & $\delta_{\mathrm{H}}($ mult, $\mathrm{Hz})$ & $\delta_{\mathrm{H}}($ mult, $\mathrm{Hz})$ & $\Delta \delta$ \\
\hline 3 & $7.87(\mathrm{~s}, 1 \mathrm{H})$ & $7.87(\mathrm{~s}, 1 \mathrm{H})$ & 0 \\
\hline 5 & $7.82(\mathrm{~s}, 1 \mathrm{H})$ & $7.83(\mathrm{~s}, 1 \mathrm{H})$ & +0.01 \\
\hline 7 & $3.44(\mathrm{~m}, 1 \mathrm{H})$ & $3.44-3.41(\mathrm{~m}, 1 \mathrm{H})$ & -0.02 \\
\hline 8 & $3.88(\mathrm{~m}, 1 \mathrm{H})$ & $3.89-3.85(\mathrm{~m}, 1 \mathrm{H})$ & -0.01 \\
\hline $\mathrm{OH} 8$ & $5.00(\mathrm{~d}, J=6.5 \mathrm{~Hz}, 1 \mathrm{H})$ & $4.98(\mathrm{~d}, J=6.5 \mathrm{~Hz}, 1 \mathrm{H})$ & -0.02 \\
\hline $9 \mathrm{a}$ & $1.64(\mathrm{~m}, 1 \mathrm{H})$ & $1.66-1.65(\mathrm{~m}, 1 \mathrm{H})$ & +0.02 \\
\hline $9 \mathrm{~b}$ & $0.43(\mathrm{dd}, J=14.3,2 \mathrm{~Hz}, 1 \mathrm{H})$ & $0.41(\mathrm{dd}, J=14.5,1.5 \mathrm{~Hz}, 1 \mathrm{H})$ & -0.02 \\
\hline 10 & $4.94(\mathrm{dd}, J=9.5,9.2 \mathrm{~Hz}, 1 \mathrm{H})$ & $4.94(\mathrm{dd}, J=9.6,9.2 \mathrm{~Hz}, 1 \mathrm{H})$ & 0 \\
\hline $\mathrm{OH} 10$ & $5.05(\mathrm{~d}, J=2.0 \mathrm{~Hz}, 1 \mathrm{H})$ & $5.07(\mathrm{~d}, J=3.0 \mathrm{~Hz}, 1 \mathrm{H})$ & +0.02 \\
\hline 11 & $5.39(\mathrm{dd}, J=15.1,9.2 \mathrm{~Hz}, 1 \mathrm{H})$ & $5.38(\mathrm{dd}, J=15.0,9.2 \mathrm{~Hz}, 1 \mathrm{H})$ & -0.01 \\
\hline 12 & $6.46(\mathrm{dd}, J=15.1,11.1 \mathrm{~Hz}, 1 \mathrm{H})$ & $6.45(\mathrm{dd}, J=15.0,11.0 \mathrm{~Hz}, 1 \mathrm{H})$ & -0.01 \\
\hline 13 & $5.87(\mathrm{dd}, J=11.0,10.0 \mathrm{~Hz}, 1 \mathrm{H})$ & $5.87(\mathrm{dd}, J=11.0,11.0 \mathrm{~Hz}, 1 \mathrm{H})$ & 0 \\
\hline 14 & $5.30(\mathrm{ddd}, J=11.4,11.4,5.4 \mathrm{~Hz}, 1 \mathrm{H})$ & $5.30(\mathrm{ddd}, J=11.3,11.3,5.4 \mathrm{~Hz}, 1 \mathrm{H})$ & 0 \\
\hline $15 \mathrm{a}$ & $2.86(\mathrm{ddd}, J=13.7,11.6,11.5 \mathrm{~Hz}, 1 \mathrm{H})$ & $2.85(\mathrm{ddd}, J=13.7,11.9,11.3 \mathrm{~Hz}, 1 \mathrm{H})$ & -0.01 \\
\hline $15 \mathrm{~b}$ & $2.24(\mathrm{dd}, J=13.7,5.1 \mathrm{~Hz}, 1 \mathrm{H})$ & $2.23(\mathrm{dd}, J=13.7,5.0 \mathrm{~Hz}, 1 \mathrm{H})$ & -0.01 \\
\hline 16 & $4.90(\mathrm{dd}, J=10.8,3.3 \mathrm{~Hz}, 1 \mathrm{H})$ & $4.90(\mathrm{dd}, J=11.2,3.3 \mathrm{~Hz}, 1 \mathrm{H})$ & 0 \\
\hline 17 & $3.57(\mathrm{dd}, J=6.8,3.5 \mathrm{~Hz}, 1 \mathrm{H})$ & $3.58(\mathrm{dd}, J=6.4,3.3 \mathrm{~Hz}, 1 \mathrm{H})$ & +0.01 \\
\hline $\mathrm{OH} 17$ & $4.82(\mathrm{~d}, J=6.0 \mathrm{~Hz}, 1 \mathrm{H})$ & $4.84(\mathrm{~d}, J=6.0 \mathrm{~Hz}, 1 \mathrm{H})$ & +0.02 \\
\hline 18 & $3.33(\mathrm{dd}, J=6.8,3 \mathrm{~Hz}, 1 \mathrm{H})$ & $3.30(\mathrm{~m}, 1 \mathrm{H})$ & -0.03 \\
\hline $\mathrm{OH} 18$ & $4.31(\mathrm{~d}, J=6.0 \mathrm{~Hz}, 1 \mathrm{H})$ & $4.32(\mathrm{~d}, J=5.8 \mathrm{~Hz}, 1 \mathrm{H})$ & +0.01 \\
\hline 19 & $1.94(\mathrm{~m}, 1 \mathrm{H})$ & $1.94-1.93(\mathrm{~m}, 1 \mathrm{H})$ & -0.01 \\
\hline $20_{a}$ & $2.12(\mathrm{dd}, J=12.9,6.5 \mathrm{~Hz}, 1 \mathrm{H})$ & $2.11(\mathrm{dd}, J=12.8,6.4 \mathrm{~Hz}, 1 \mathrm{H})$ & -0.01 \\
\hline $20_{b}$ & $1.77(\mathrm{dd}, J=12.9,7.8 \mathrm{~Hz}, 1 \mathrm{H})$ & $1.76(\mathrm{dd}, J=12.8,8.0 \mathrm{~Hz}, 1 \mathrm{H})$ & -0.01 \\
\hline 22 & $5.59(\mathrm{~s}, 1 \mathrm{H})$ & $5.58(\mathrm{~s}, 1 \mathrm{H})$ & -0.01 \\
\hline 24 & $5.22(\mathrm{q}, J=7.0 \mathrm{~Hz}, 1 \mathrm{H})$ & $5.24(\mathrm{q}, J=6.8 \mathrm{~Hz}, 1 \mathrm{H})$ & +0.02 \\
\hline 25 & $1.59(\mathrm{~d}, J=7.0 \mathrm{~Hz}, 3 \mathrm{H})$ & $1.58(\mathrm{~d}, J=6.8 \mathrm{~Hz}, 3 \mathrm{H})$ & -0.01 \\
\hline 26 & $1.95(\mathrm{~s}, 3 \mathrm{H})$ & $1.95(\mathrm{~s}, 3 \mathrm{H})$ & 0 \\
\hline 32 & $3.94(\mathrm{~m}, 2 \mathrm{H})$ & $3.94(\mathrm{~m}, 2 \mathrm{H})$ & 0 \\
\hline $\mathrm{OH} 32$ & $5.17(\mathrm{t}, J=4.3 \mathrm{~Hz}, 1 \mathrm{H})$ & $5.17(\mathrm{t}, J=4.6 \mathrm{~Hz}, 1 \mathrm{H})$ & 0 \\
\hline 33 & $0.76(\mathrm{~d}, J=6.5 \mathrm{~Hz}, 3 \mathrm{H})$ & $0.75(\mathrm{~d}, J=6.7 \mathrm{~Hz}, 3 \mathrm{H})$ & -0.01 \\
\hline 34 & $1.70(\mathrm{~s}, 3 \mathrm{H})$ & $1.70(\mathrm{~s}, 3 \mathrm{H})$ & 0 \\
\hline 35 & $1.63(\mathrm{~s}, 3 \mathrm{H})$ & $1.63(\mathrm{~s}, 3 \mathrm{H})$ & 0 \\
\hline
\end{tabular}


Table S2. Comparison of ${ }^{13}$ C-NMR Spectral Data between Natural and Synthetic Thuggacin cmc-A (1)

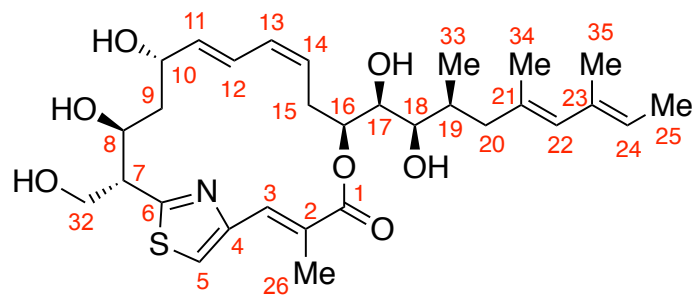

thuggacin cmc-A (1)

\begin{tabular}{|c|c|c|c|}
\hline & $\begin{array}{l}\text { Natural Thuggacin cmc-A (1) } \\
{ }^{13} \mathrm{C}(150 \mathrm{MHz})^{7}\end{array}$ & $\begin{array}{l}\text { Synthetic Thuggacin cmc-A (1) } \\
{ }^{13} \mathrm{C}(125 \mathrm{MHz})\end{array}$ & $\begin{array}{l}\Delta \delta \text { (synthetic 1- } \\
\text { natural) ppm }\end{array}$ \\
\hline Position & $\delta_{\mathrm{C}}($ mult, $\mathrm{Hz})$ & $\delta_{\mathrm{C}}($ mult, $\mathrm{Hz})$ & $\Delta \delta$ \\
\hline 1 & 166.9 & 166.9 & 0 \\
\hline 2 & 126.0 & 126.0 & 0 \\
\hline 3 & 133.5 & 133.5 & 0 \\
\hline 4 & 149.0 & 149.0 & 0 \\
\hline 5 & 121.0 & 121.1 & +0.1 \\
\hline 6 & 168.0 & 168.0 & 0 \\
\hline 7 & 51.4 & 51.4 & 0 \\
\hline 8 & 70.1 & 70.1 & 0 \\
\hline 9 & 39.3 & 39.2 & -0.1 \\
\hline 10 & 69.6 & 69.5 & -0.1 \\
\hline 11 & 137.2 & 137.2 & 0 \\
\hline 12 & 125.0 & 125.0 & 0 \\
\hline 13 & 131.0 & 131.0 & 0 \\
\hline 14 & 128.0 & 128.1 & +0.1 \\
\hline 15 & 30.0 & 30.0 & 0 \\
\hline 16 & 74.3 & 74.2 & -0.1 \\
\hline 17 & 72.1 & 72.1 & 0 \\
\hline 18 & 71.8 & 71.8 & 0 \\
\hline 19 & 32.3 & 32.3 & 0 \\
\hline 20 & 45.2 & 45.3 & +0.1 \\
\hline 21 & 133.3 & 133.3 & 0 \\
\hline 22 & 130.2 & 130.2 & 0 \\
\hline 23 & 133.3 & 133.3 & 0 \\
\hline 24 & 122.6 & 122.7 & $\begin{array}{r}+0.1 \\
\end{array}$ \\
\hline 25 & 13.5 & 13.5 & 0 \\
\hline 26 & 13.4 & 13.4 & 0 \\
\hline 32 & 59.8 & 59.8 & 0 \\
\hline 33 & 13.6 & 13.7 & +0.1 \\
\hline 34 & 17.7 & 17.7 & 0 \\
\hline 35 & 16.7 & 16.7 & 0 \\
\hline
\end{tabular}




\section{Reference}

(1) Shiina, I.; Kikuchi, T.; Sasaki, A. The first total synthesis of (-) and (+)-2-hydroxy-24oxooctacosanolide using an effective lactonization. Org. Lett., 2006, 21, 4995-4958.

(2) Park, S. H.; Yoon, H. K.; Lee, H. W. Metal-mediated diastereoselective allylation reaction of chiral $\alpha, \beta$-epoxy aldehyde. part 2. Bull. Korean Chem. Soc., 2008, 29, 1663-1664.

(3) Bock, M.; Dehn, R.; Kirschning, A. Total synthesis of thuggacin B. Angew. Chem. Int. Ed. 2008, 47, 9134-9137.

(4) (a) Evans, D. A.; Rieger, D. L.; Jones, T. K.; Kaldor, S. W. Assignment of stereochemistry in the oligomycin/rutamycin/cytovaricin family of antibiotics. Asymmetric synthesis of the rutamycin spiroketal synthon. J. Org. Chem. 1990, 55, 6260-6268. (b) For a procedure for the synthesis of the enantiomeric chiral propionimide, see: Gage, J. R.; Evans, D. A. Org. Synth. 1989, 68, 83-91.

(5) Davies, J. A.; Bull, F. M.; Walker, P. D.; Weir, A. N. M.; Lavigne, R.; Masschelein, J.; Simpson, T. J.; Race, P. R.; Crump, M. P.; Willis, C. L. Total synthesis of kalimantacin A. Org. Lett. 2020, 22, 6349-6353.

(6) Forsyth, C. J.; Xu, J.; Nguyen, S. T.; Samdal, I. A.; Briggs, L. R.; Rundberget, T.; Sandvik, M.; Miles, C. O. J. Am. Chem. Soc. 2006, 128, 15114-15116.

(7) Steinmetz, H.; Irschik, H.; Kunze, B.; Reichenbach, H.; Höfle, G.; Jansen, R. Thuggacins, macrolide antibiotics active against mycobacterium tuberculosis: isolation from myxobacteria, structure elucidation, conformation analysis and biosynthesis. Chem. Eur. J. 2007, 13, 5822-5832. 


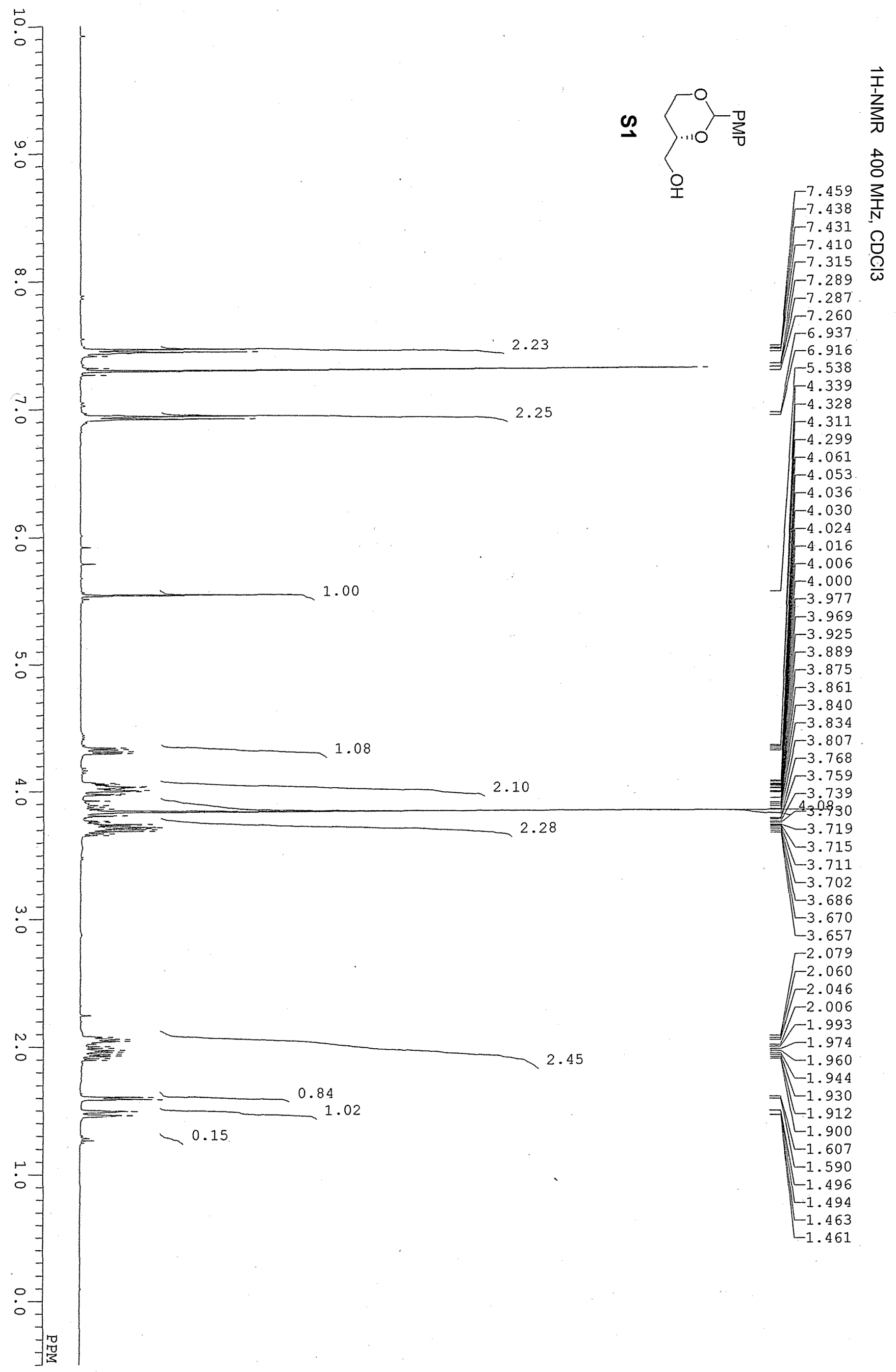




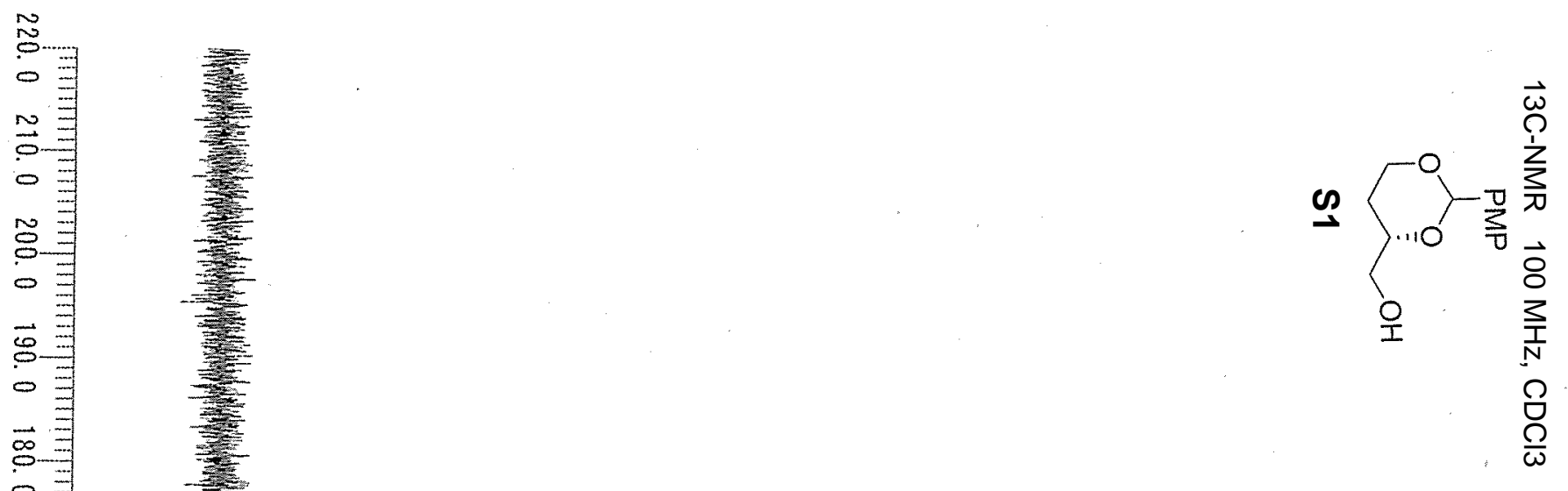

130. 891

127. 384

$\overrightarrow{0}$

○

$\vec{\theta}$

$\circ$

$\overrightarrow{0}$

s.

$\overrightarrow{0}$

$\overrightarrow{0}$

○

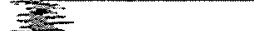

$-113 \cdot-603$

$-101.155$

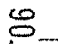

0

$\infty$

0

$\checkmark$

0

9

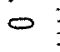

si

$\circ$

s.

0

$\infty$

$\circ$

응

$\circ$

$\overrightarrow{0}$

。寻
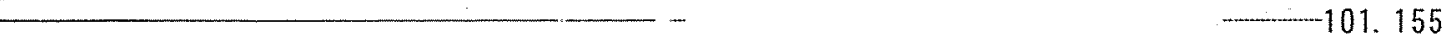

ق

翟

( 


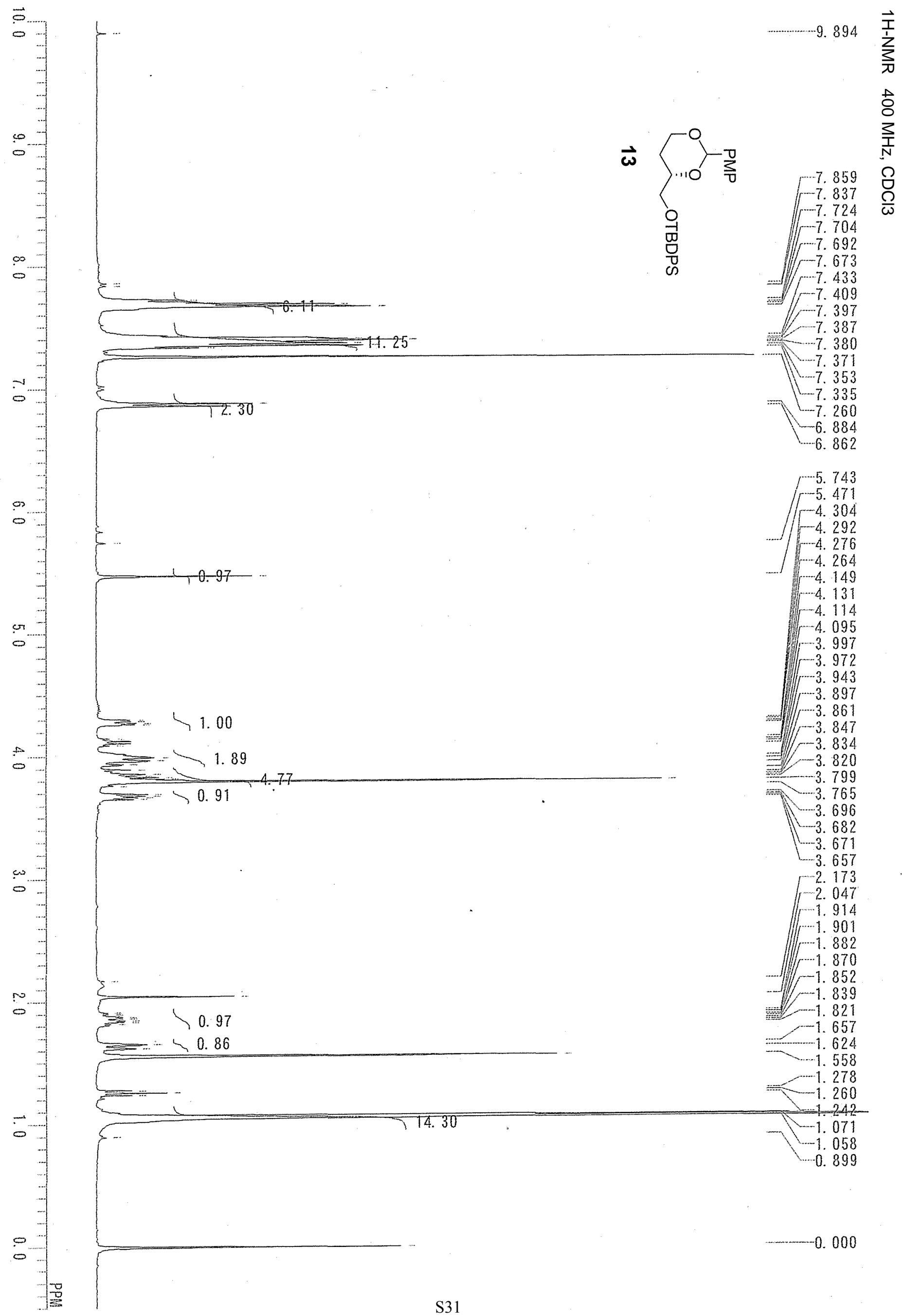




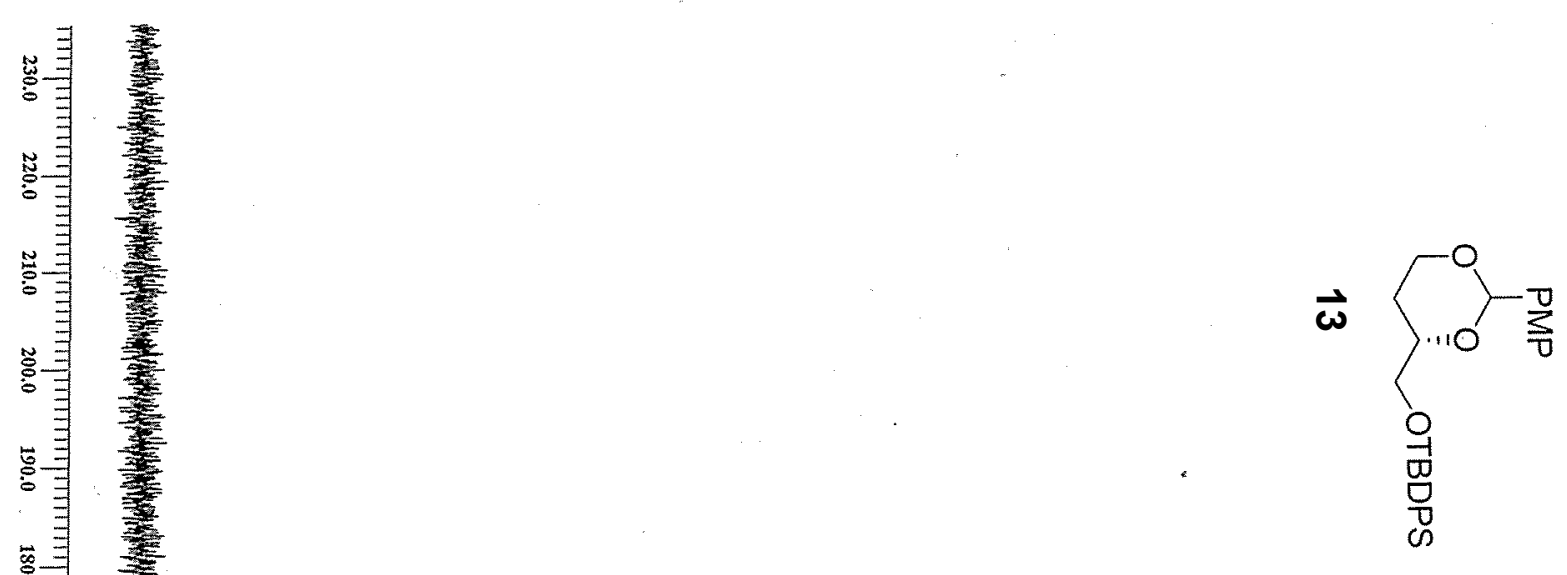
129.6069 127.6040 127.6064
-127.3677 


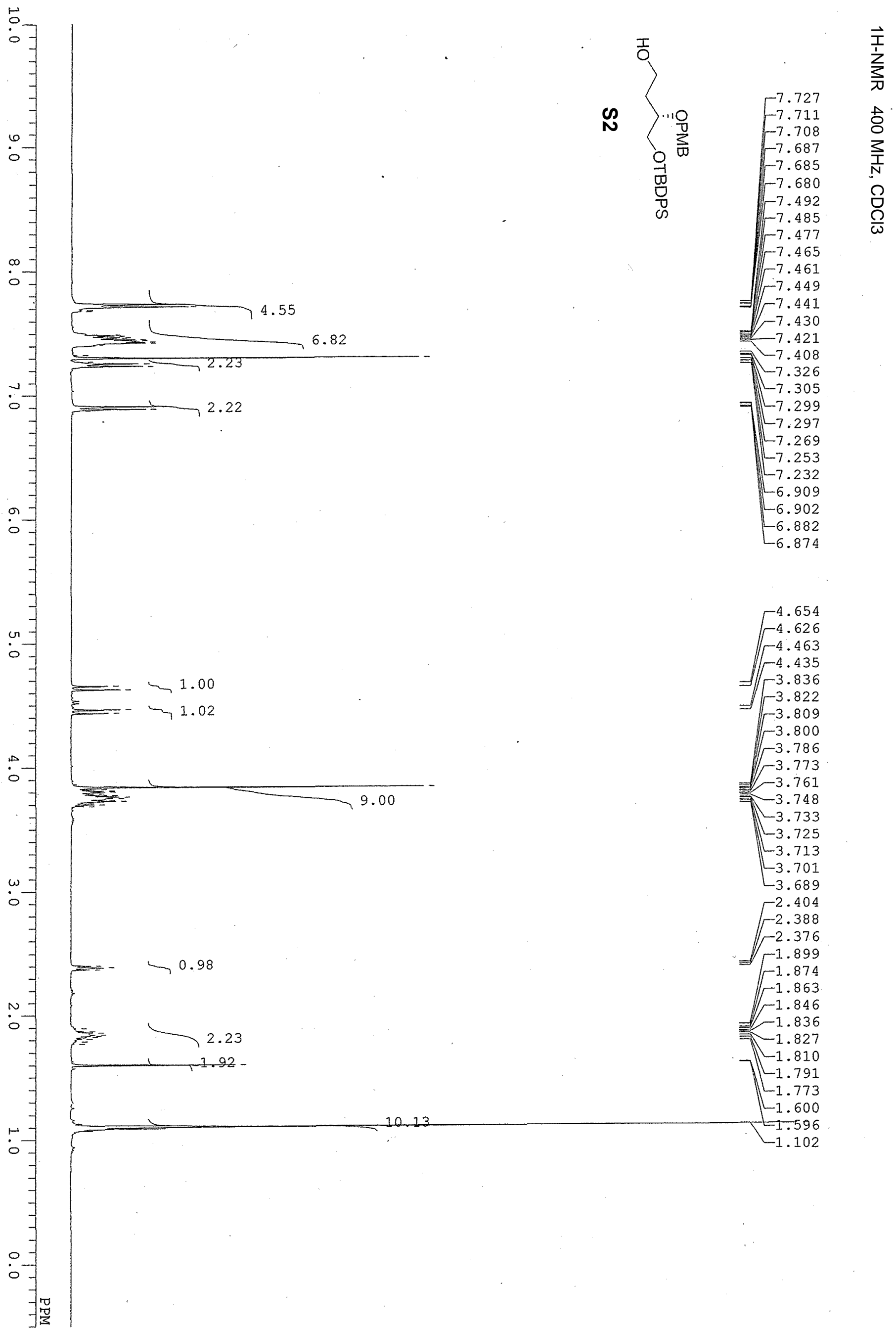




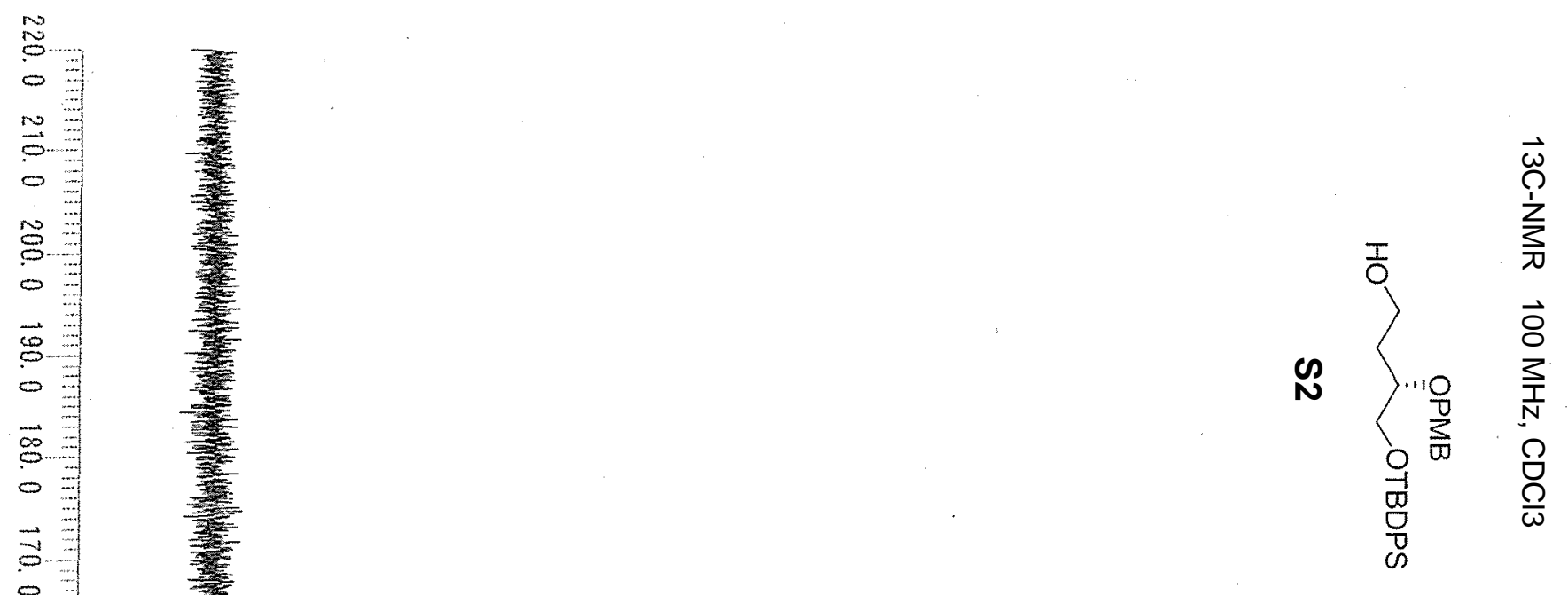

$-\quad 159.212$

क्ष

cํㅇ

$\circ$

D

$-135.600$

-135. 570

-135.295
-133.295

$-133.213$ $-130.422$

$=-129.739$

$-129.451$

127.713

113.817

$\overrightarrow{\vec{\sigma}}$

跍

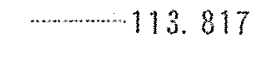

8

0

9

$\circ$

$\infty$

78. 408

77. 313

-77.000

$-76.679$

71. 797

65. 878

60. 452

55. 225

34. 141

$-26.797$

19. 157

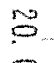

$\overline{0}$

$\infty$

$\infty$

$\frac{1}{0} .9$ 


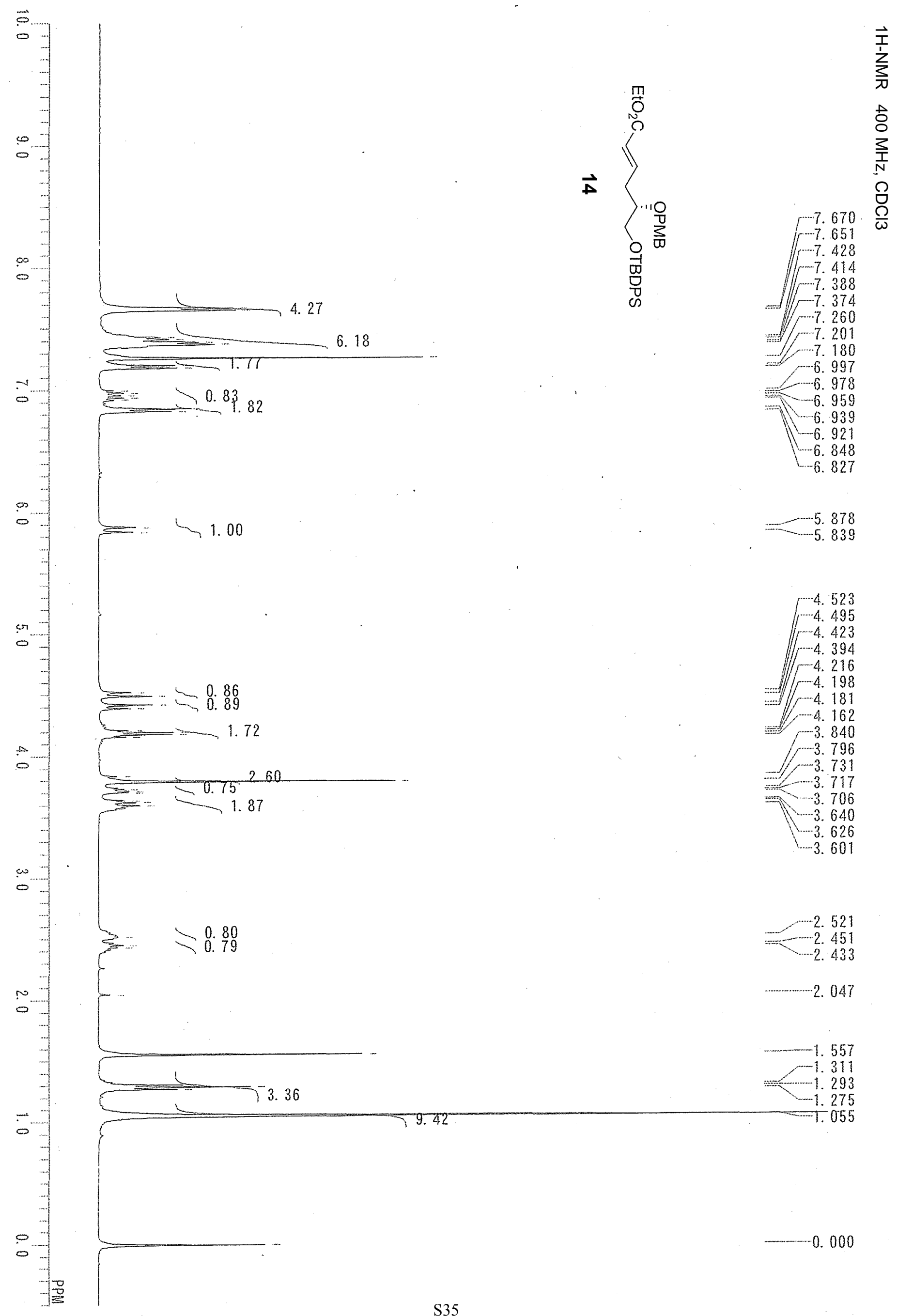




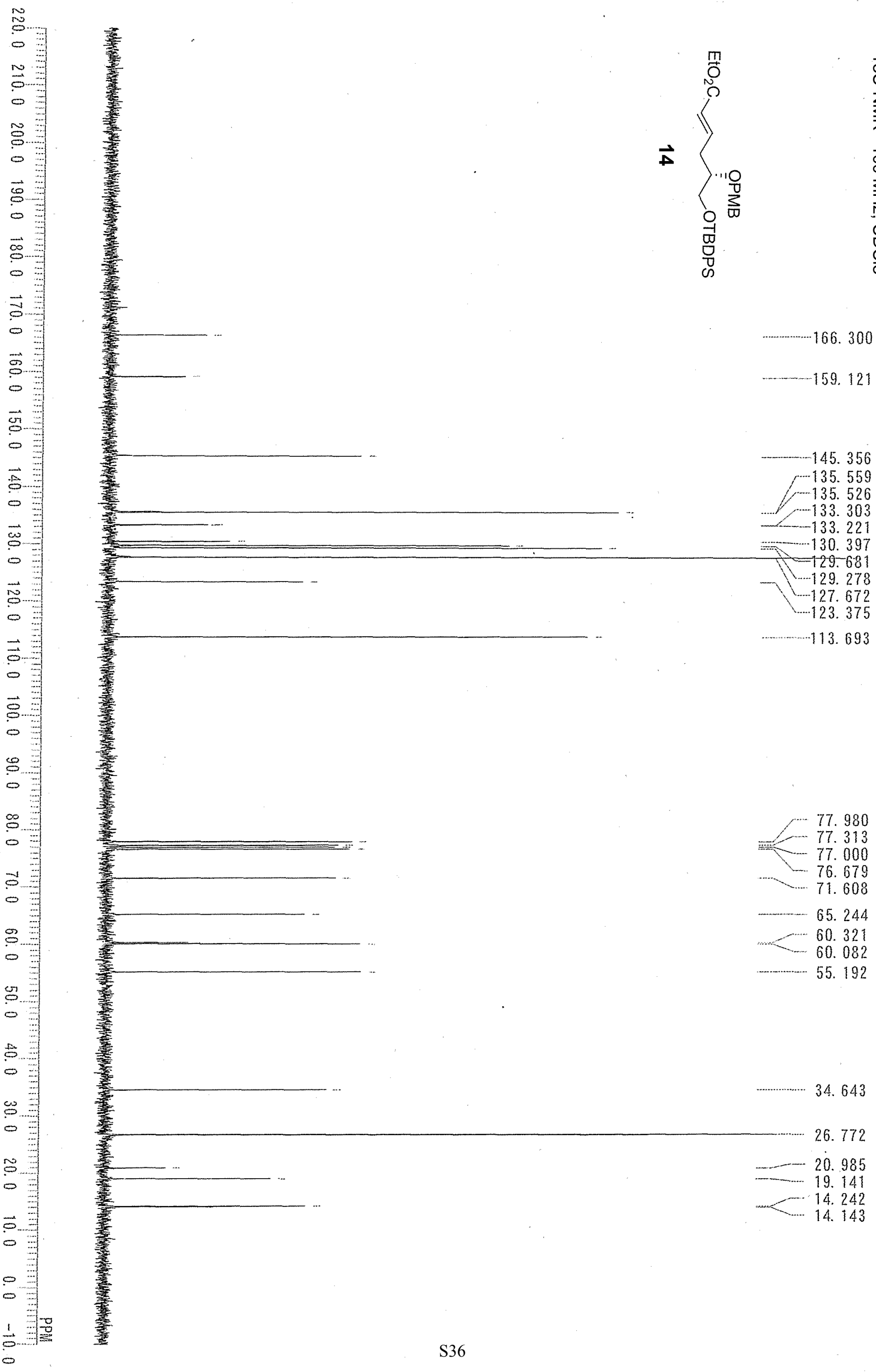




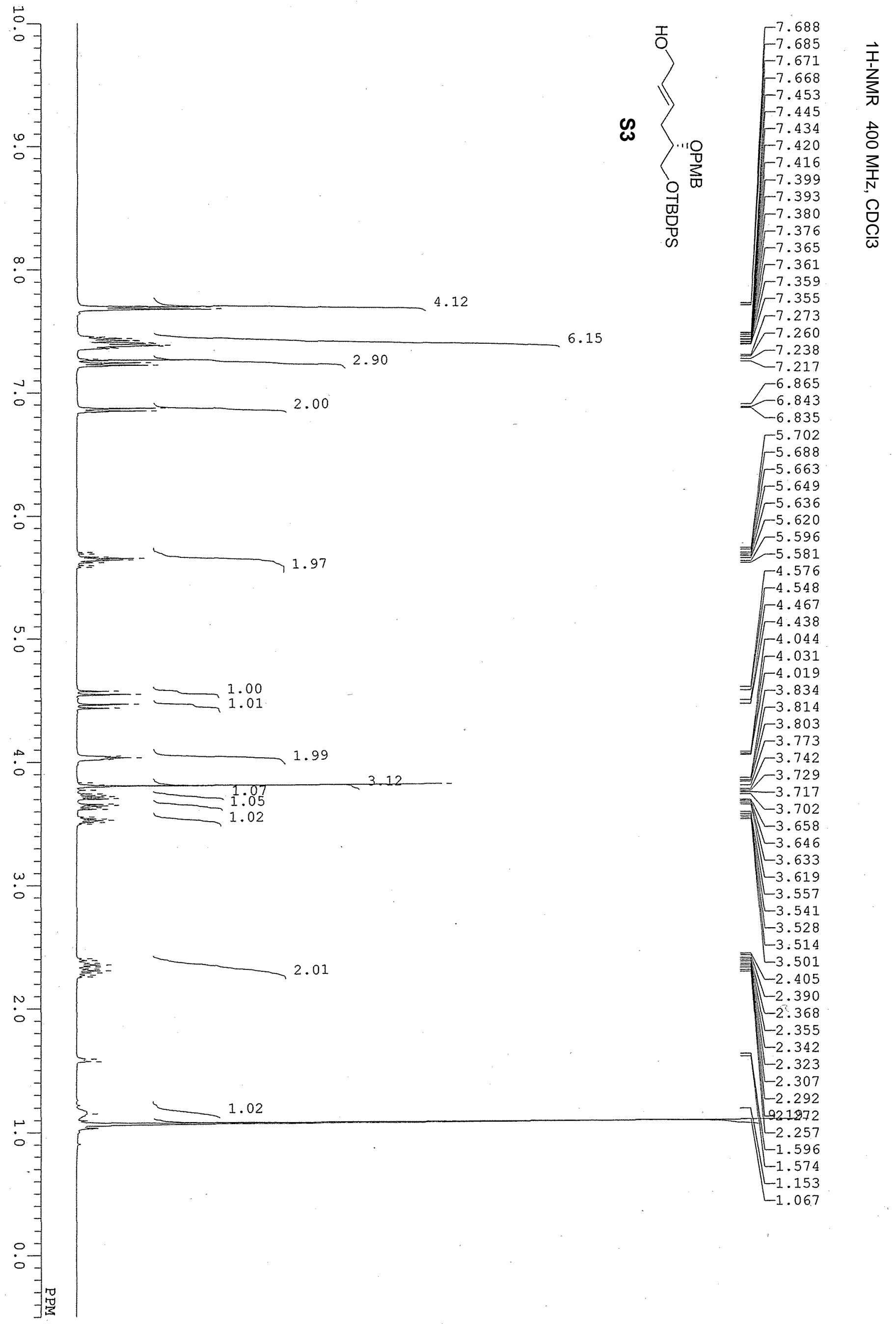




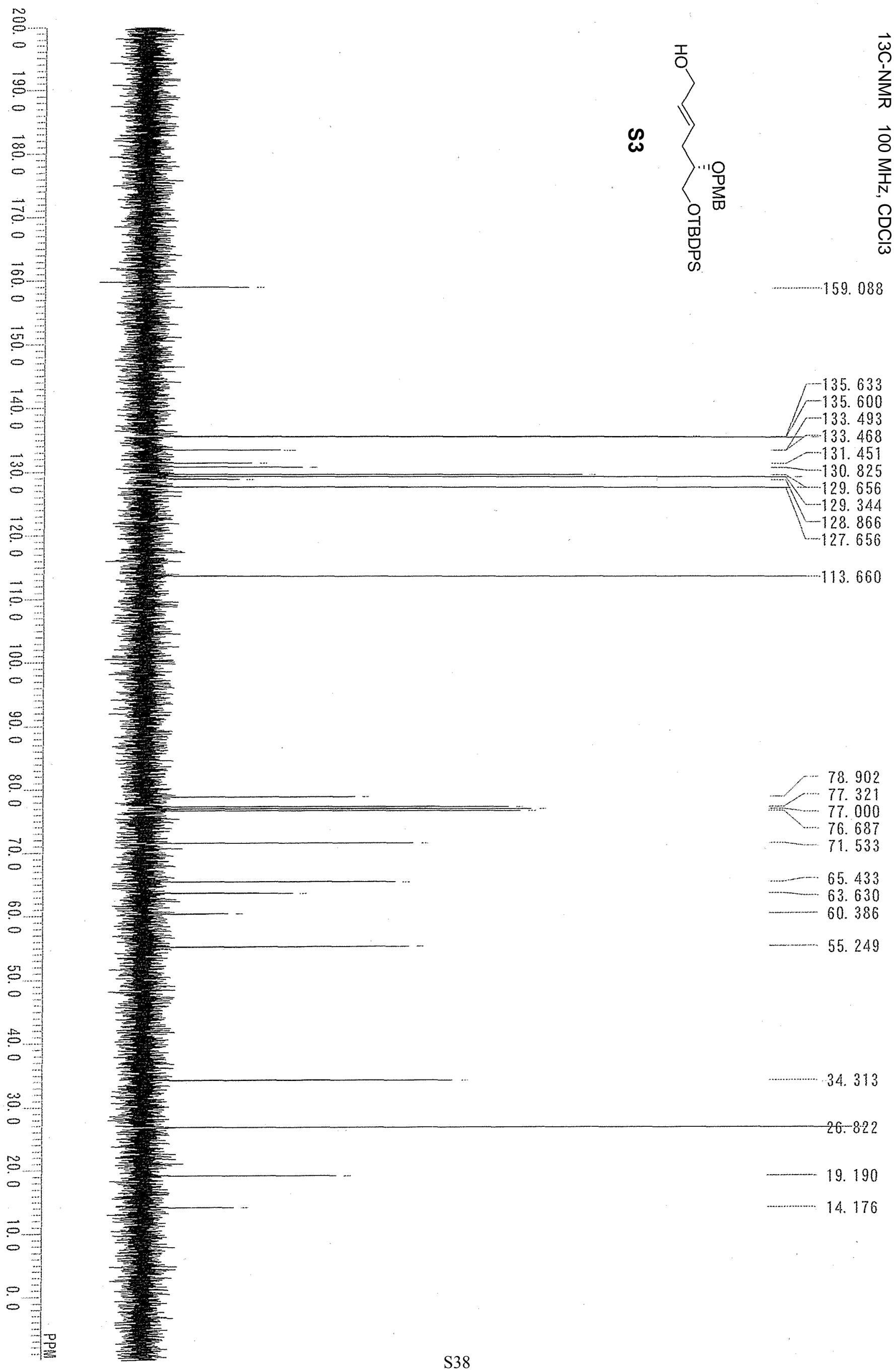


0

so

0

0

0

$-2$

0

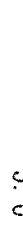

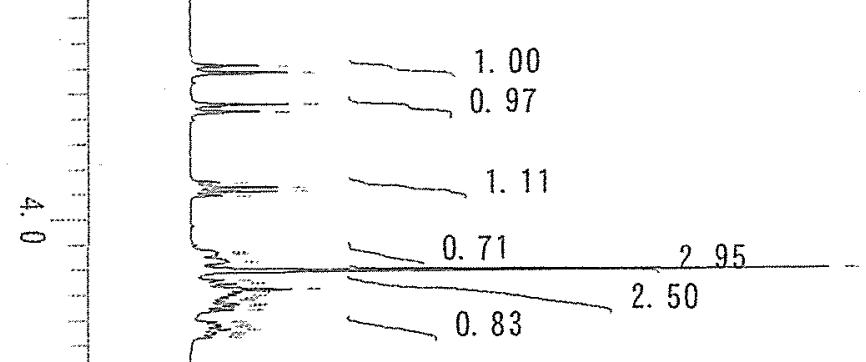

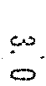

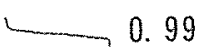

$\underline{5}$

$\longrightarrow 1.01$

2. 07

5. 01

3. 98
6. 44

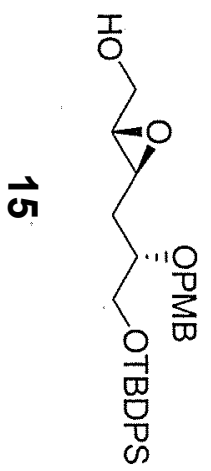

7.676

-7. 660

$-7.452$

-7. 434

7. 419

7. 394

7. 379

7. 358

$-7.261$

$-7.227$

$-7.205$

-862
-6.862

$-6.841$

$-4.613$

$-4.586$

$-4.459$

$-4.431$

-4.149
-4.139

4. 131

$-4.114$

$-4.095$

$-3.871$

$-3.864$

$-3.833$

-3. 798

$-3.759$

$-3.747$

$-3.724$

$-3.713$

-3. 702

$-3.692$

$-3.681$

$-3.667$

- 3.657

$-3.645$

- 3.594

3.583
-3.576
-3.564

- -3.564

$-3.552$

(-3. 3.545

$-3.534$

-3.085

$-3.073$

$-3.061$

$-2.925$

-2. 920

2. 048

$-1.856$

$-1.821$

$-1.808$

-1.808
-1.801

$-1.787$

$1-1.778$

$-1.770$

$-1.762$

$-1.753$

$-1.570$

$=1.536$

-1. 521

$-1.278$

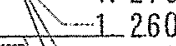

$\sqrt{-1.243}$

I-1. 212

$-1.057$

$-1.019$

-... 0.900

0.884

-.. 0.866

0.000

0 


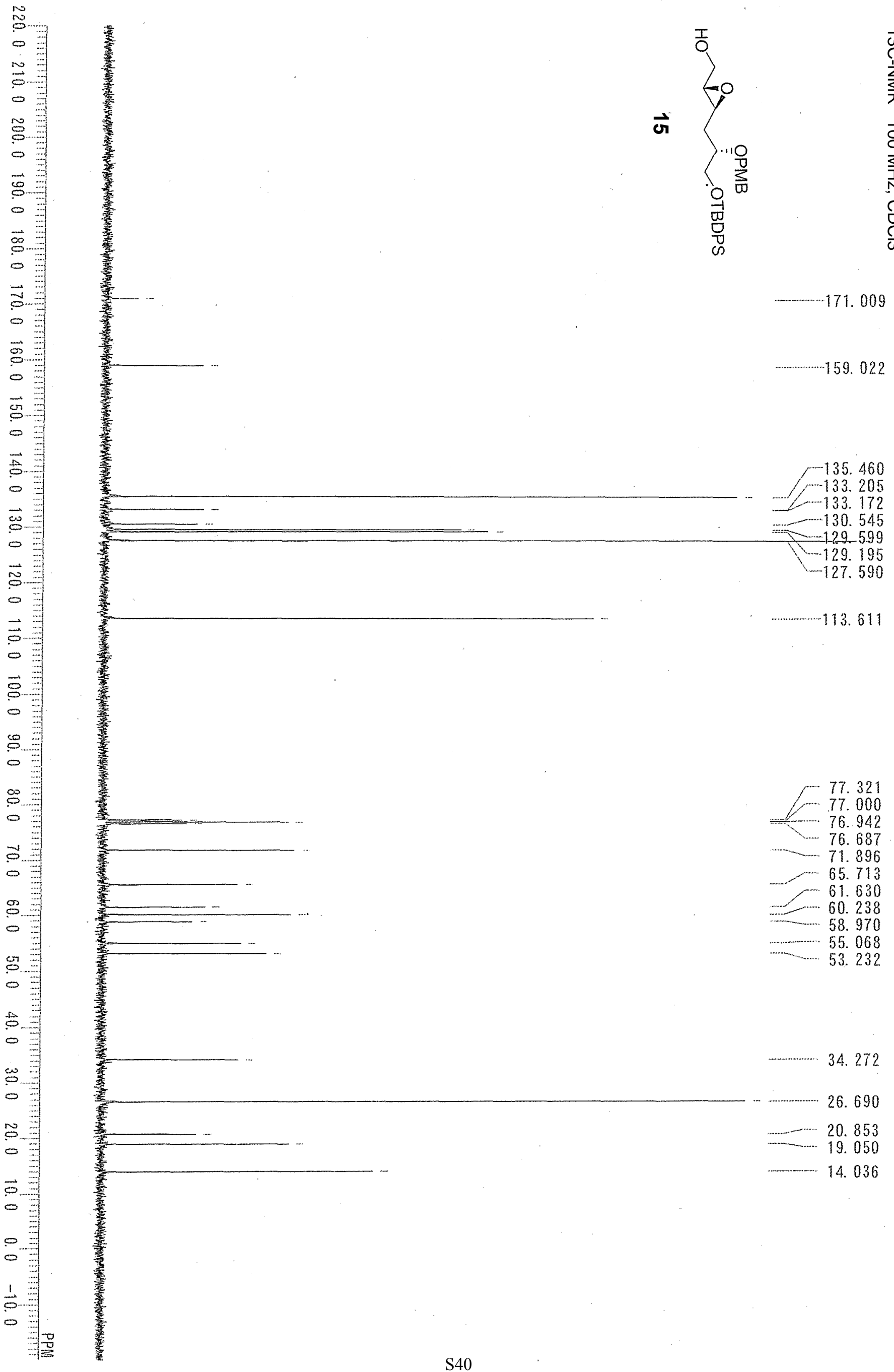




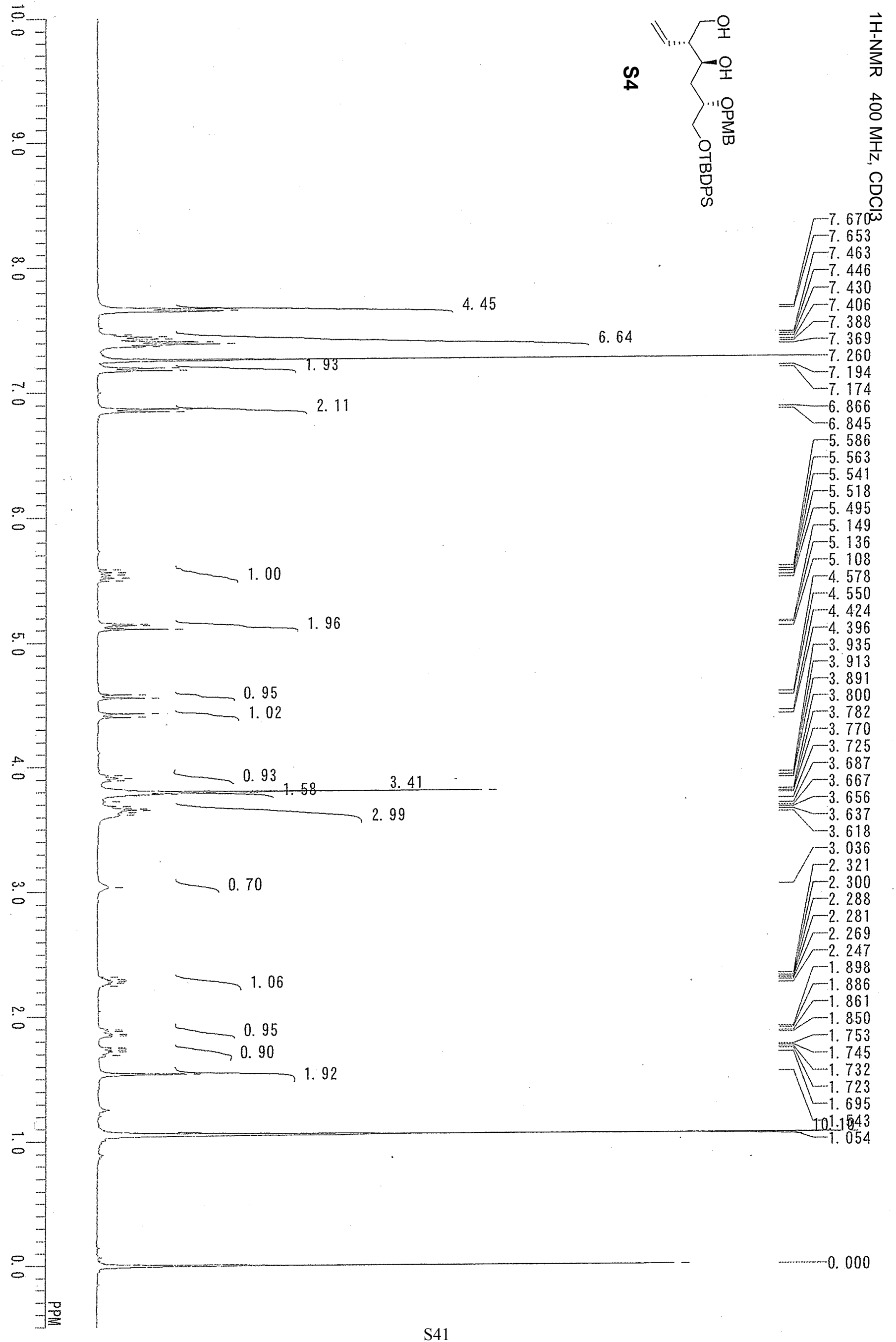




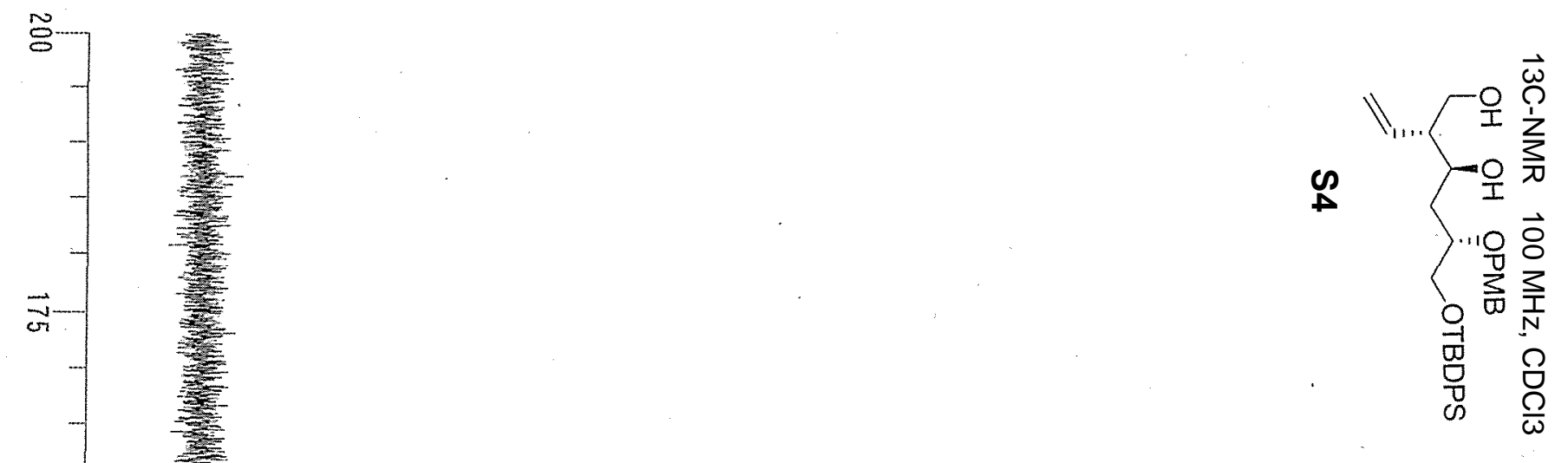

ज̆

जิ

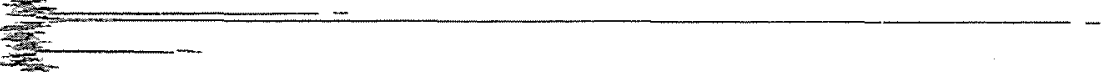

136. 086

$-135.567$

$-133.188$

$-133.106$

129. 944

$-129.805$

$-129.615$

27. 746

$-118.048$

$-113.907$

동

$\overrightarrow{8}$
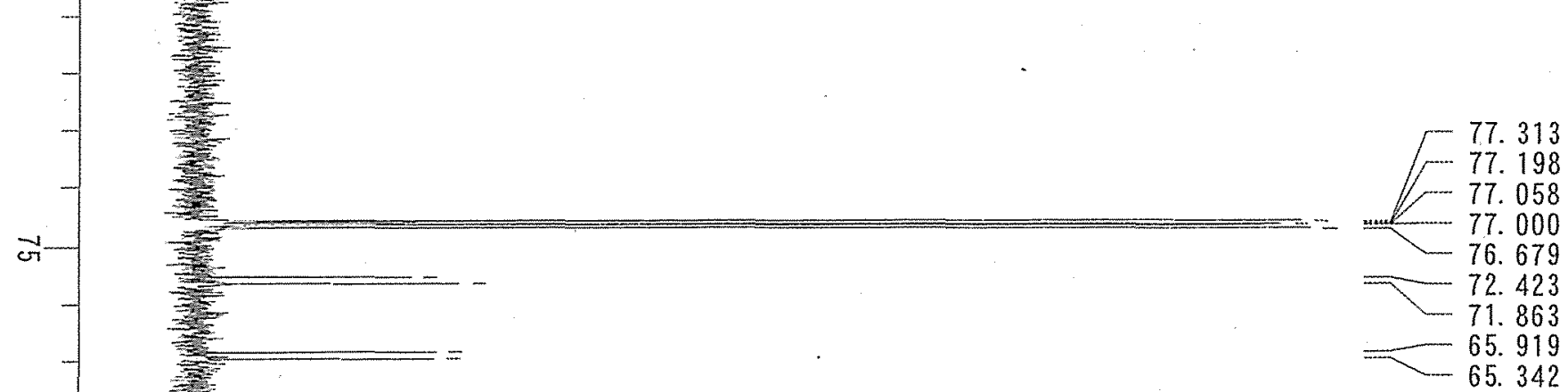

55. 266

51. 750

g

36. 001

26. 781

N

19. 141 


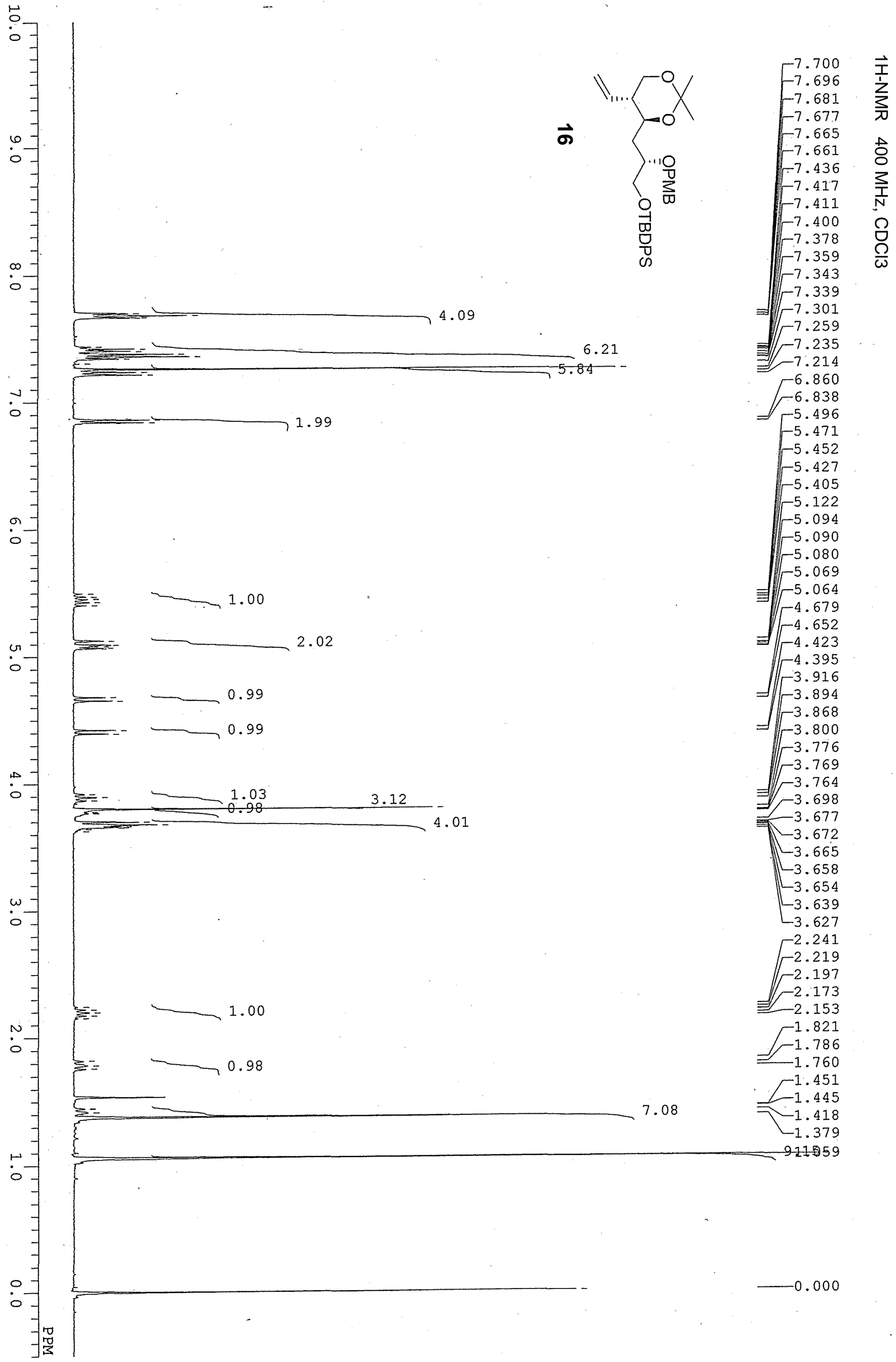




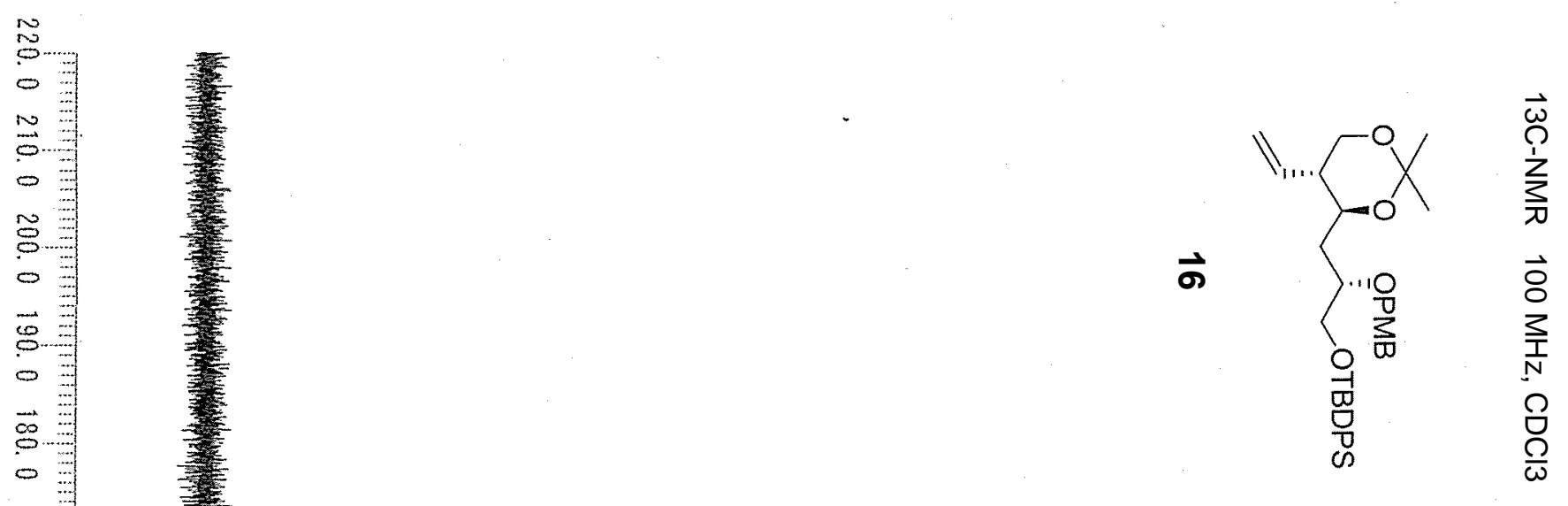

159.031

98. 051

c

0

$\infty$

77. 313

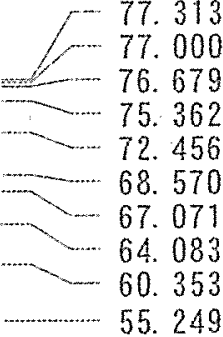

읭

s

0

s.

135.666

$-135.625$

134. 678

$-133.550$

131.278

$-129.294$

127.606

118. 625

113.685

$\overrightarrow{8}$

51

0

0

8
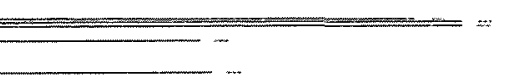

76. 679

75. 362

456

64. 083

55. 249

45. 831

36. 429

29. 785

26. 830

21. 009

19. 190

19. 116

14. 168 


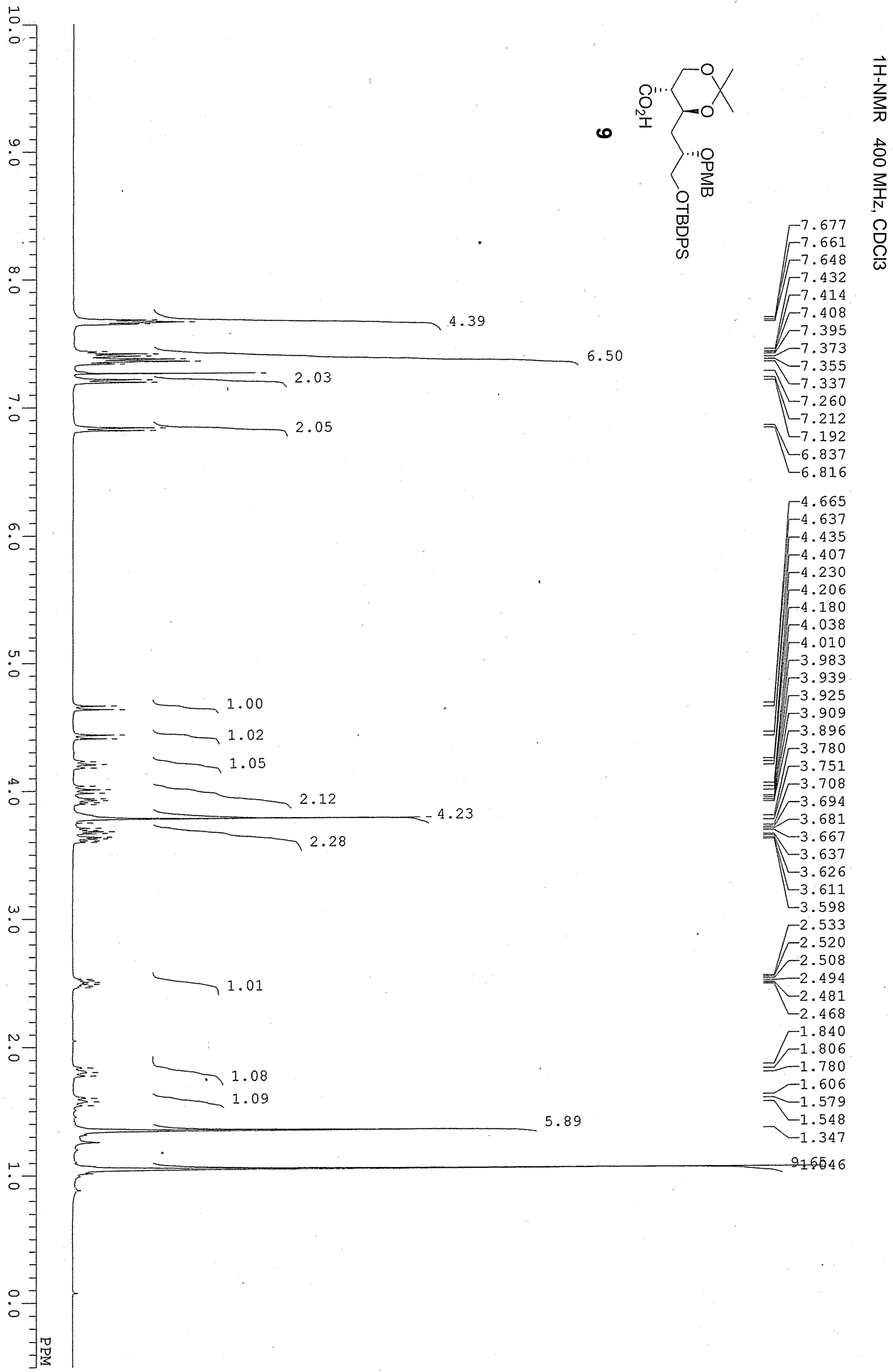




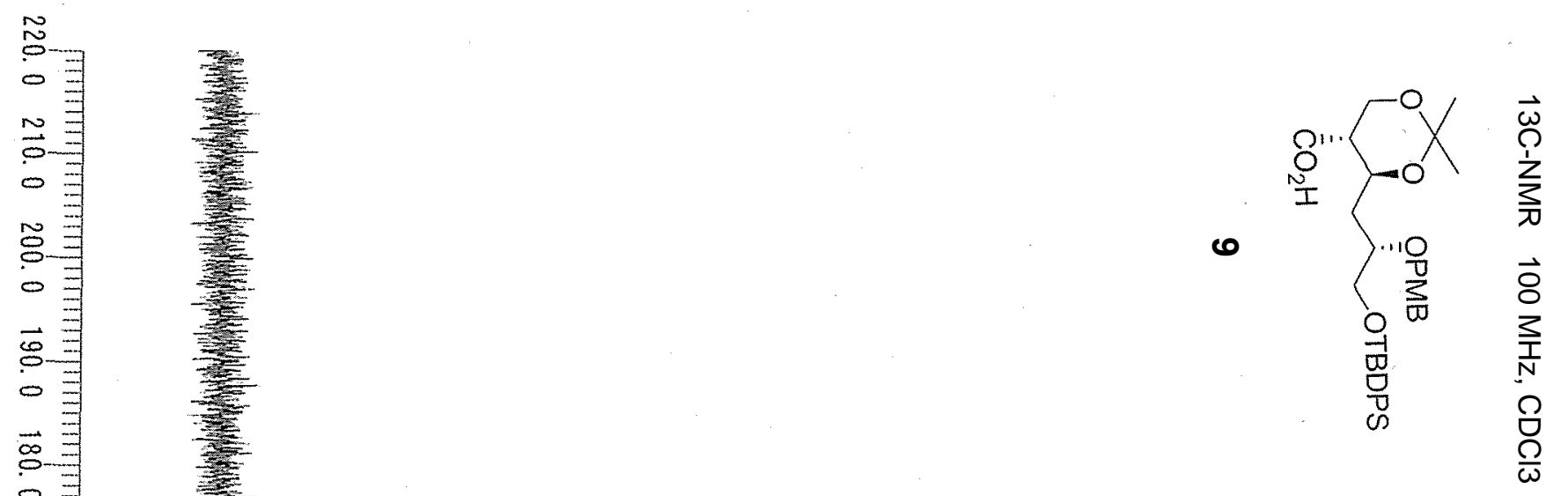

$-159.195$

ज़ㅣㅁ

$\vec{b}$

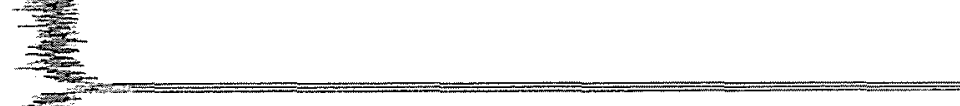

98. 504

뭉

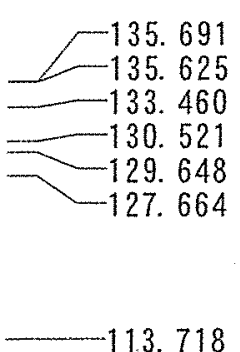

- 7. 7.321

$-77.000$

$-75.246$

72. 349

66. 709

66. 380

61.152

55. 257

46. 951

37. 302

29. 217

26. 855

-19.256
$+\quad 19.182$ 


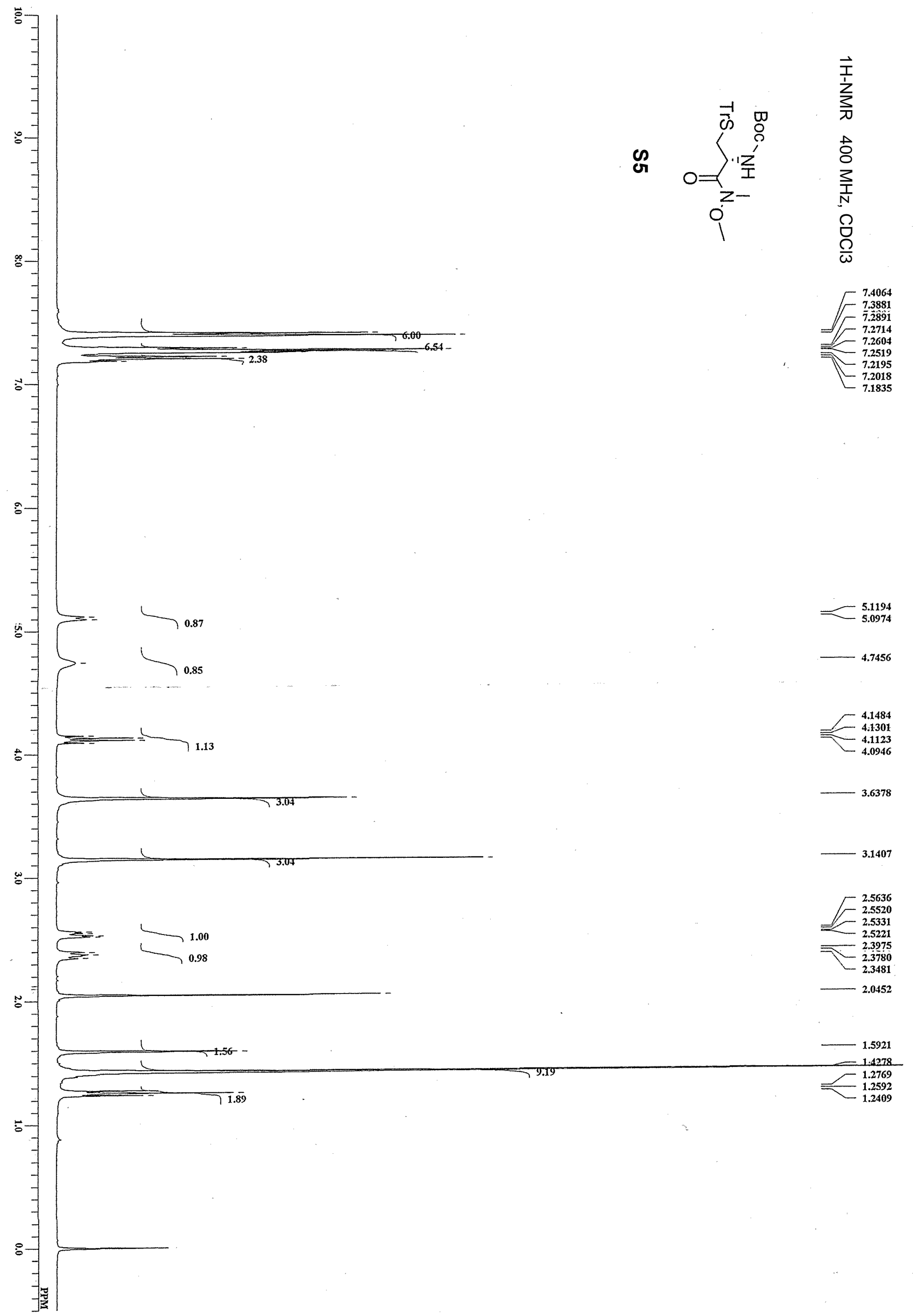




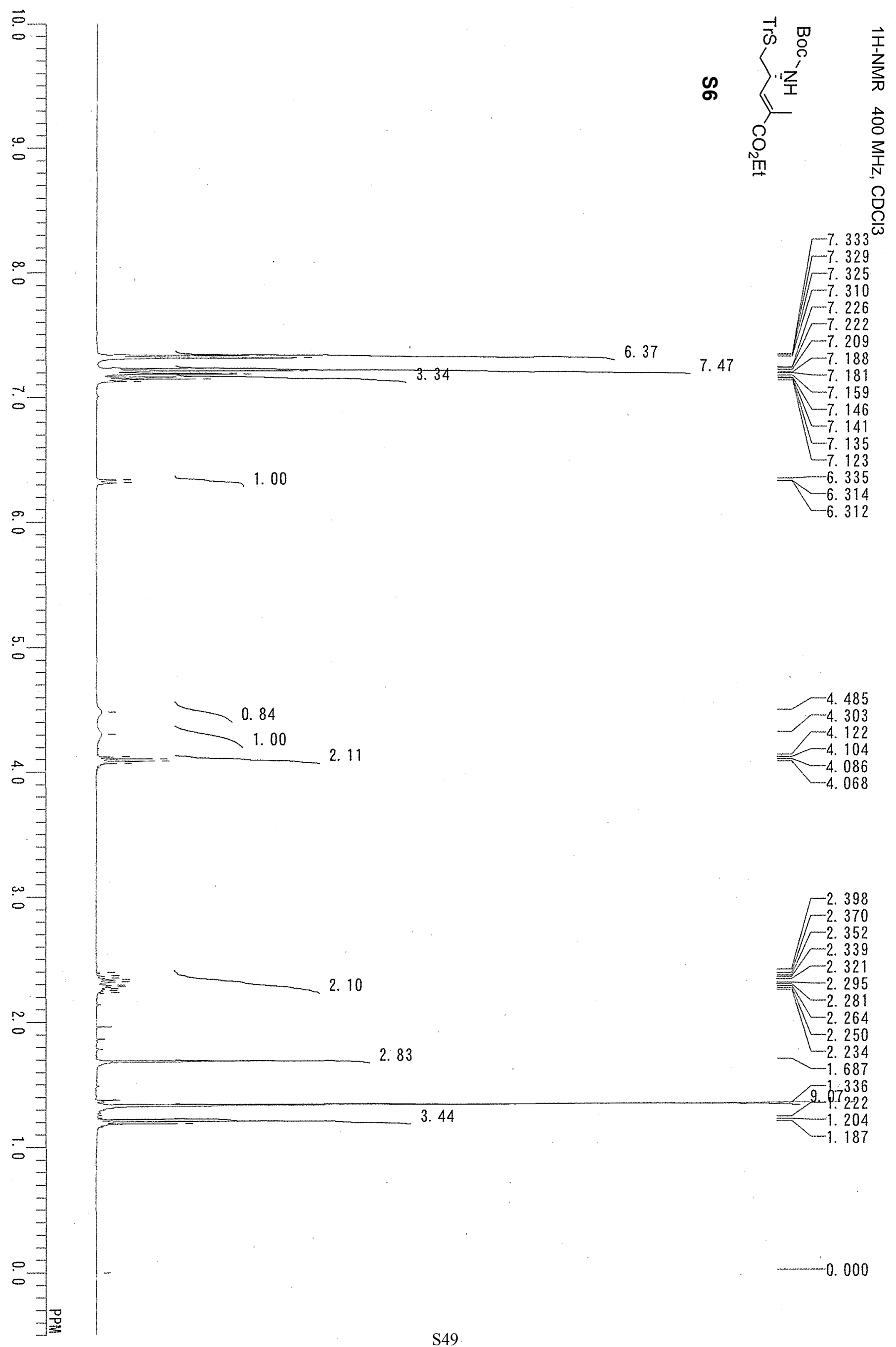


No

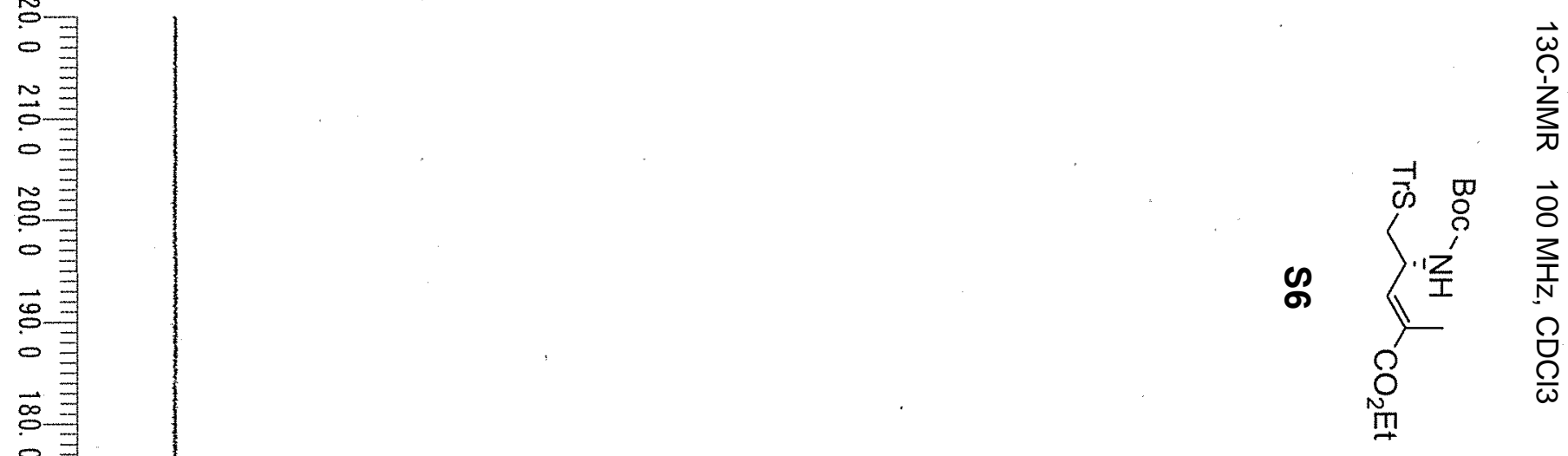

$\vec{D}$

$-167.379$

$-154.618$

몽

$\overrightarrow{0}$

$\vec{\omega}$

0

$\mathbb{N}$

0

$\overrightarrow{0}$

$\circ$

$\overrightarrow{8}$

0

0

0

$\stackrel{\infty}{0}$

¿

0

9

0

g

0

$-144.434$

-144. 302

$-139.832$

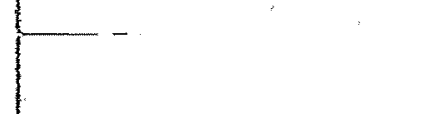

129. 360

$-127.763$

127.705

126.618

$-126.520$

79. 338

77. 321

77.000

76.679

$-66.808$

60. 502

60.115

$-48.186$

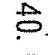

$\infty$

$\omega$

$\infty$

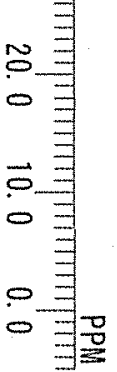

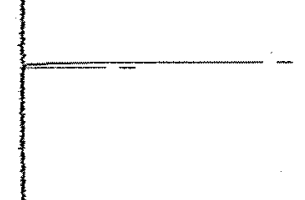

36. 182

28.155

28.057

$-14.020$

13. 987

12. 629 


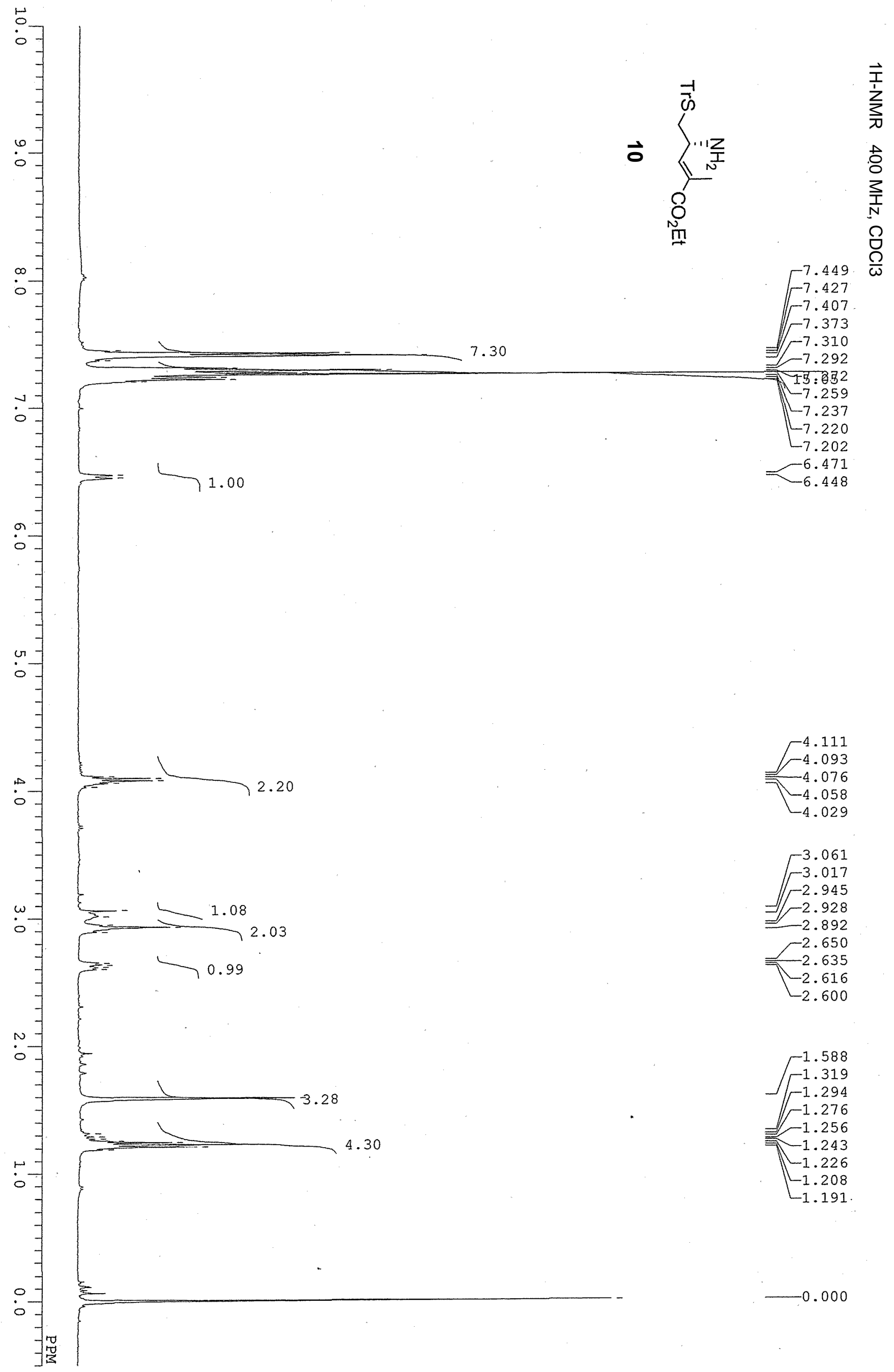




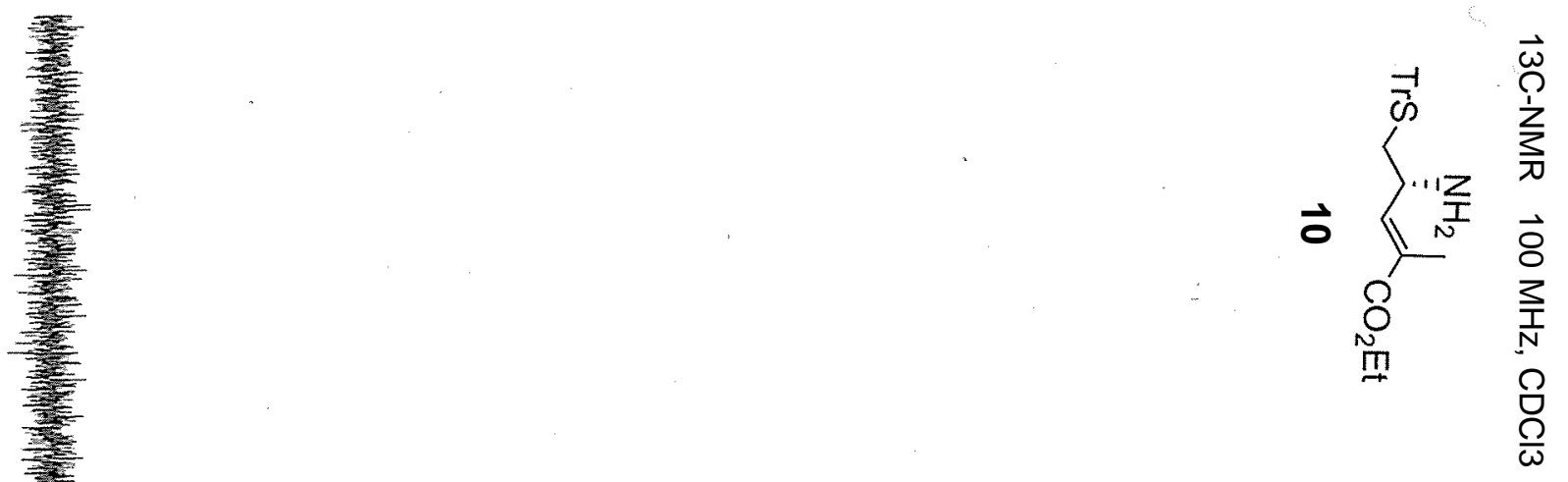




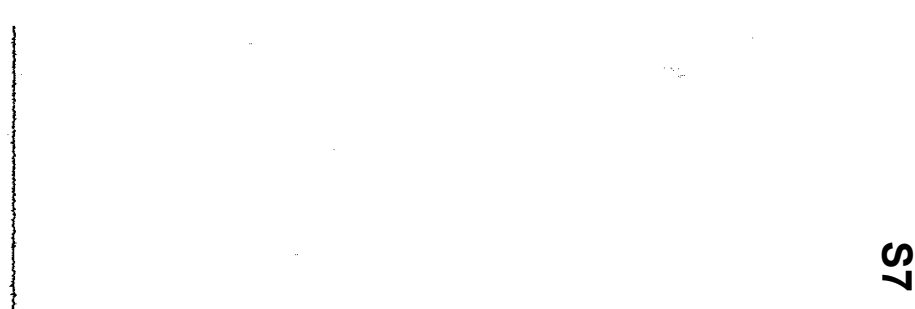

-1
-1
$0-1$
$0-1$
-1
-1
-1
$\infty-1$
$0-1$

s

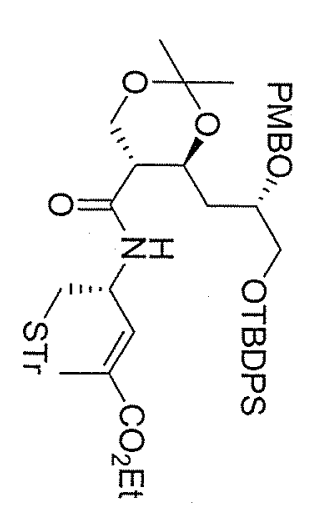

$-7.689$

$-7.338$

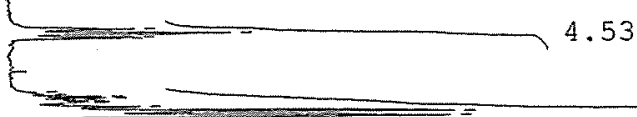

$\dot{0}$

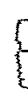

=
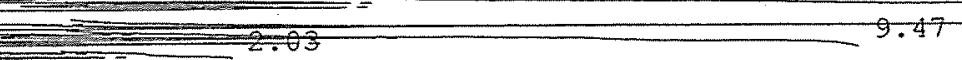

9.47

${ }^{7} .276$

12.62 릴 $14: 26$

$-7.160$

$7\lfloor-7.148$

$-7.143$

$-7.137$

$-6.834$

$7-6.813$

$-6.283$

$-6.280$

$-6.261$

$-6.257$

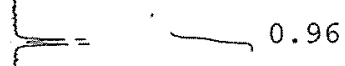

$-5.566$

$-5.548$

4.651

$-4.623$

$-4.561$

$-4.539$

G

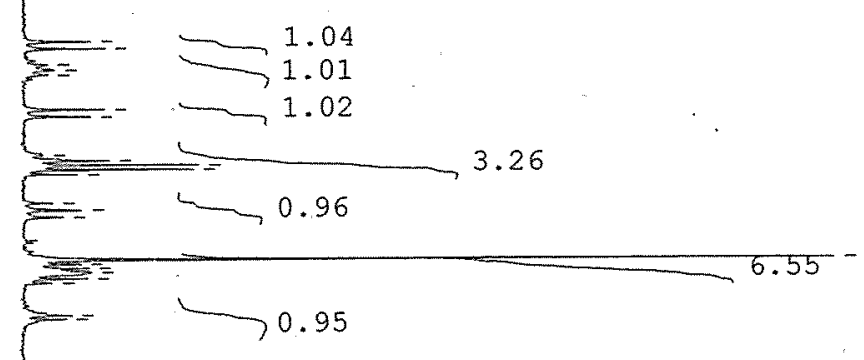

$=-4.520$

$\Rightarrow-4.383$

$-4.355$

$\mapsto$

$-4.201$

:

$\Rightarrow$

$-4.161$

$-4.144$

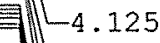

4.012

$-3.983$

$-3.955$

$-3.785$

$-3.768$

w

$-3.751$

$-3.735$

$-3.709$

$-3.694$

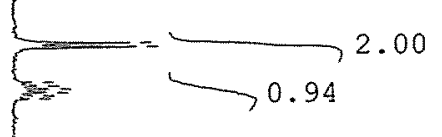

$-3.563$

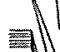

$-3.548$

N

3.26

$-2.327$

$-2.188$

$-2.173$

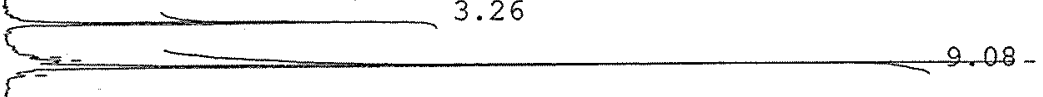

6.58

$-2.161$

$-2.149$

$-2.134$

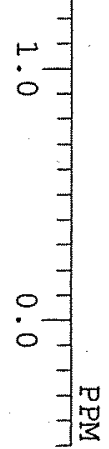

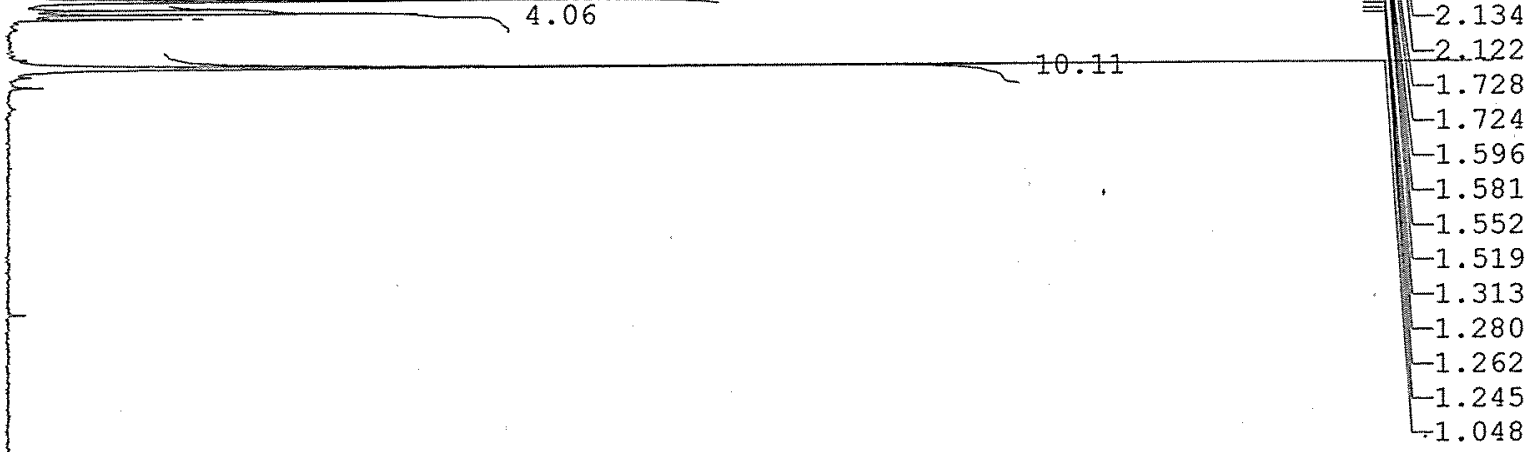



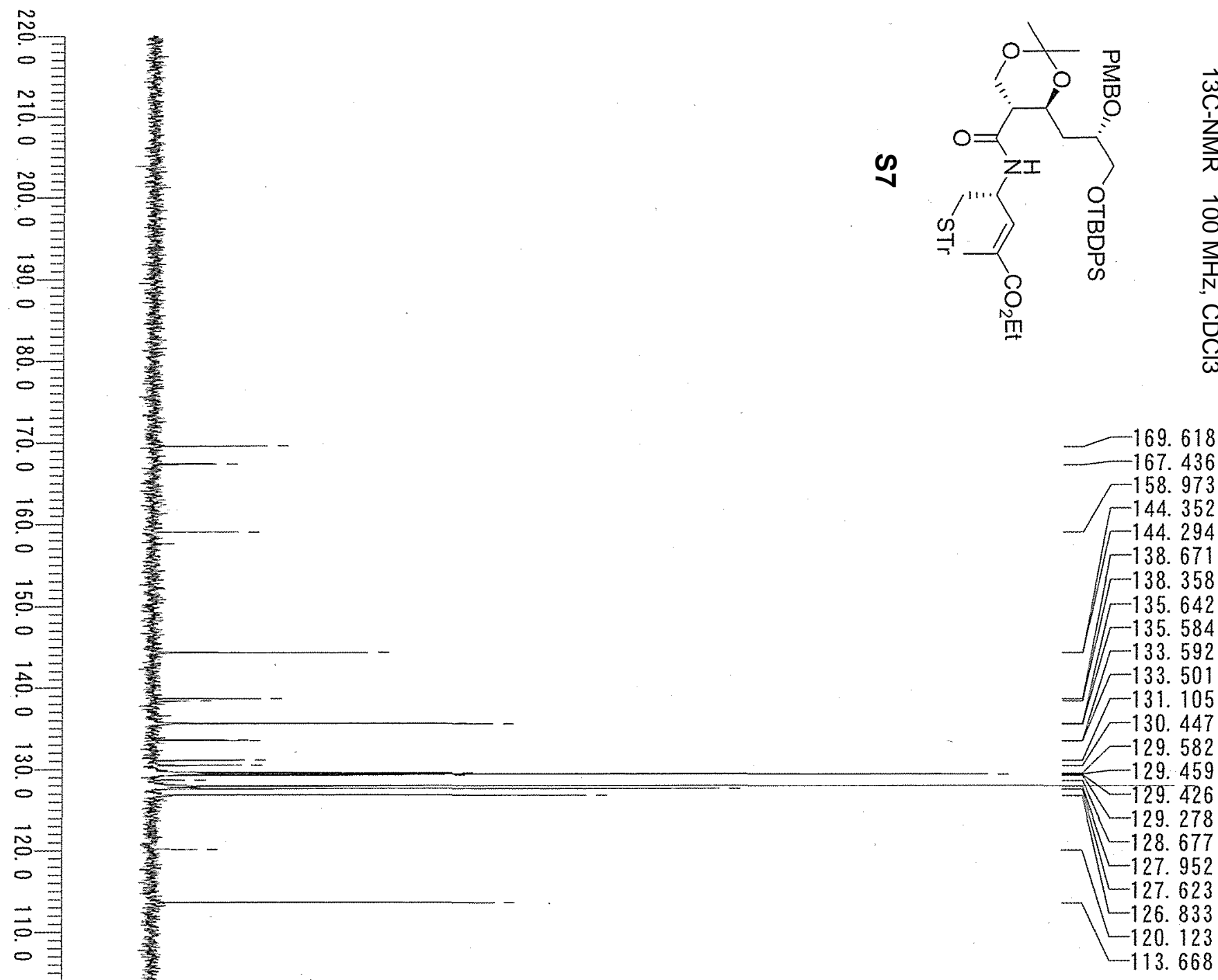

98. 37.2
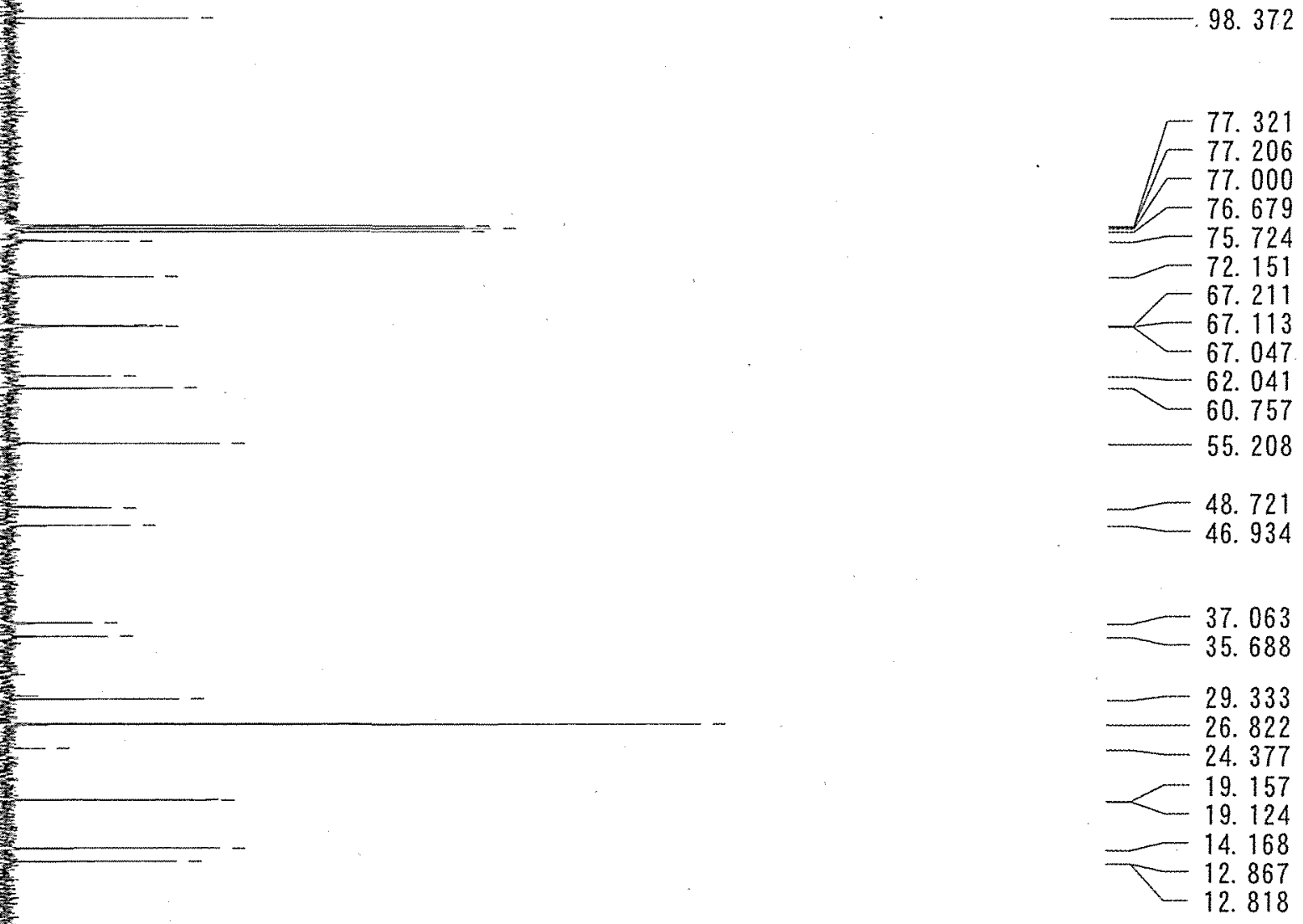


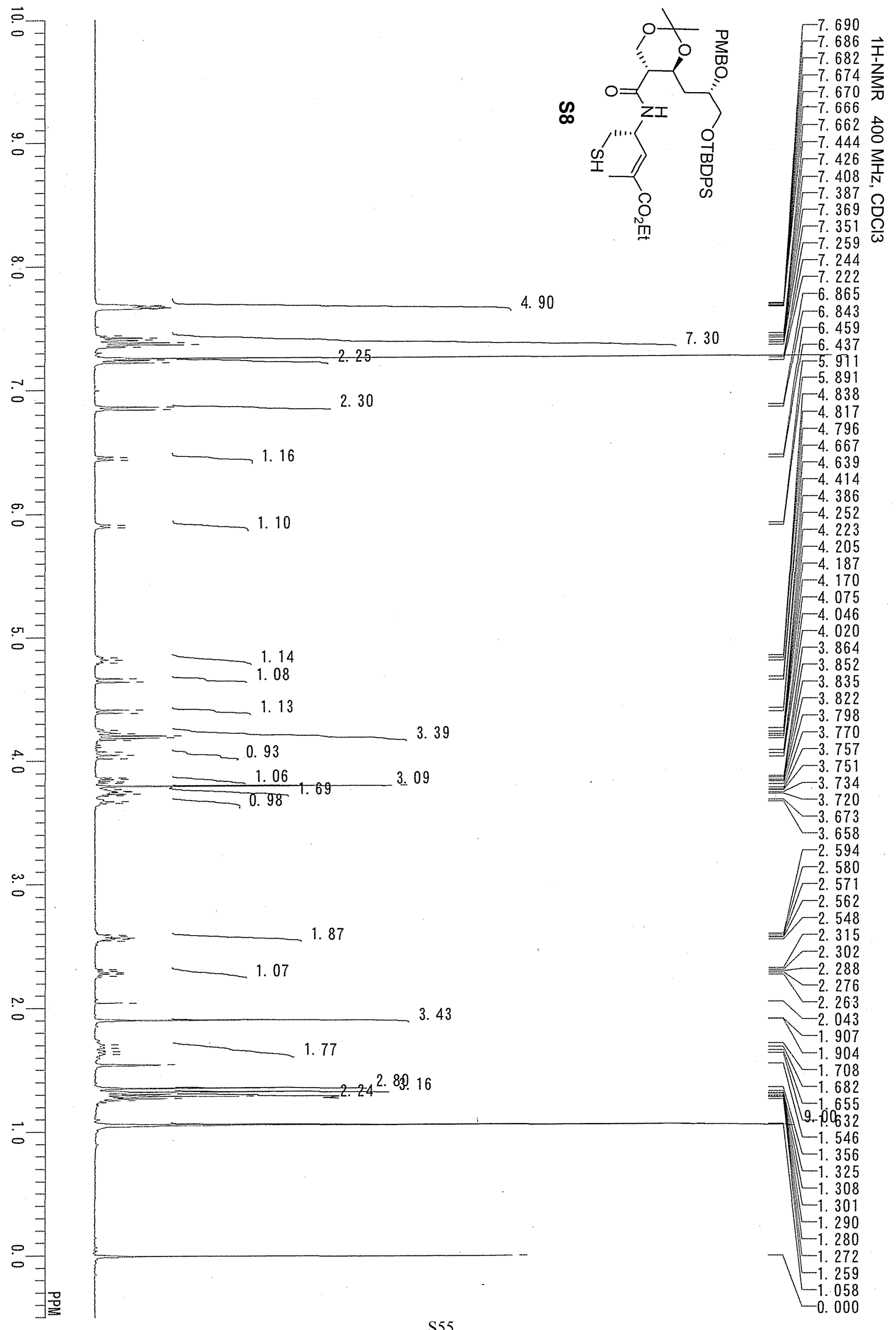




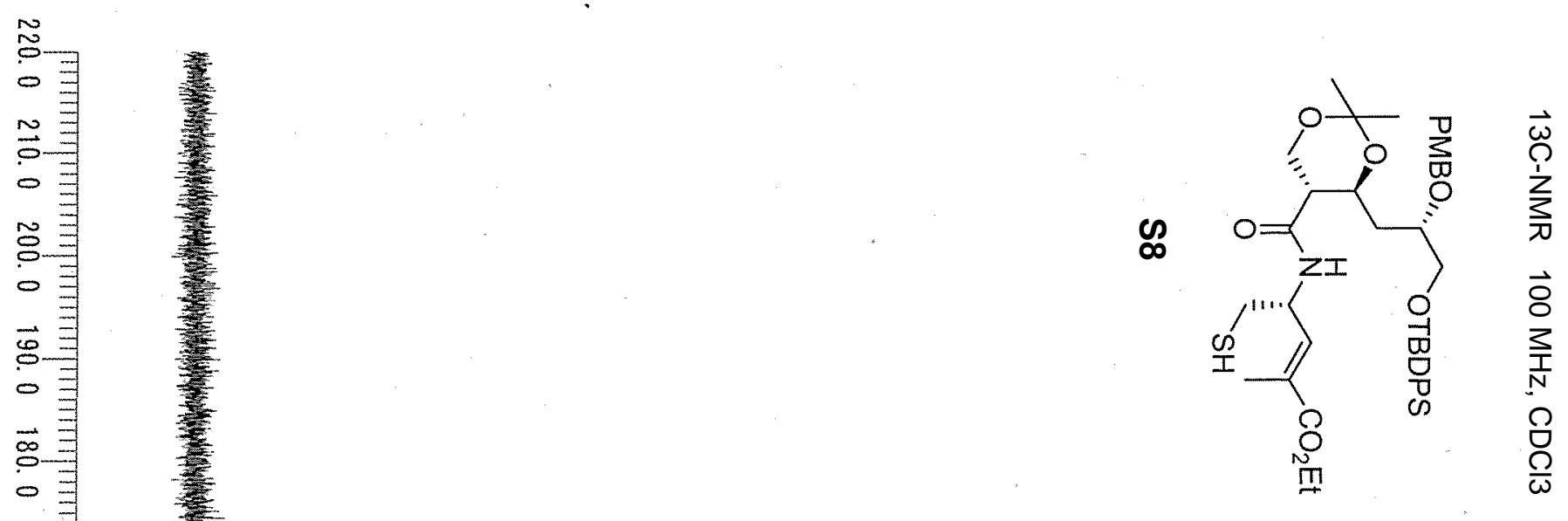

\section{$-$}

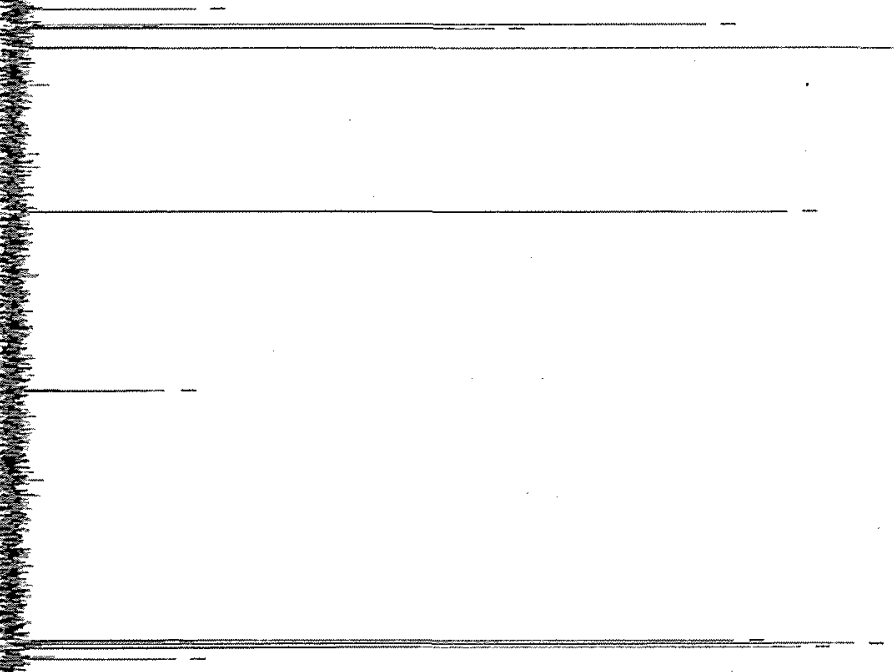

98. 504 


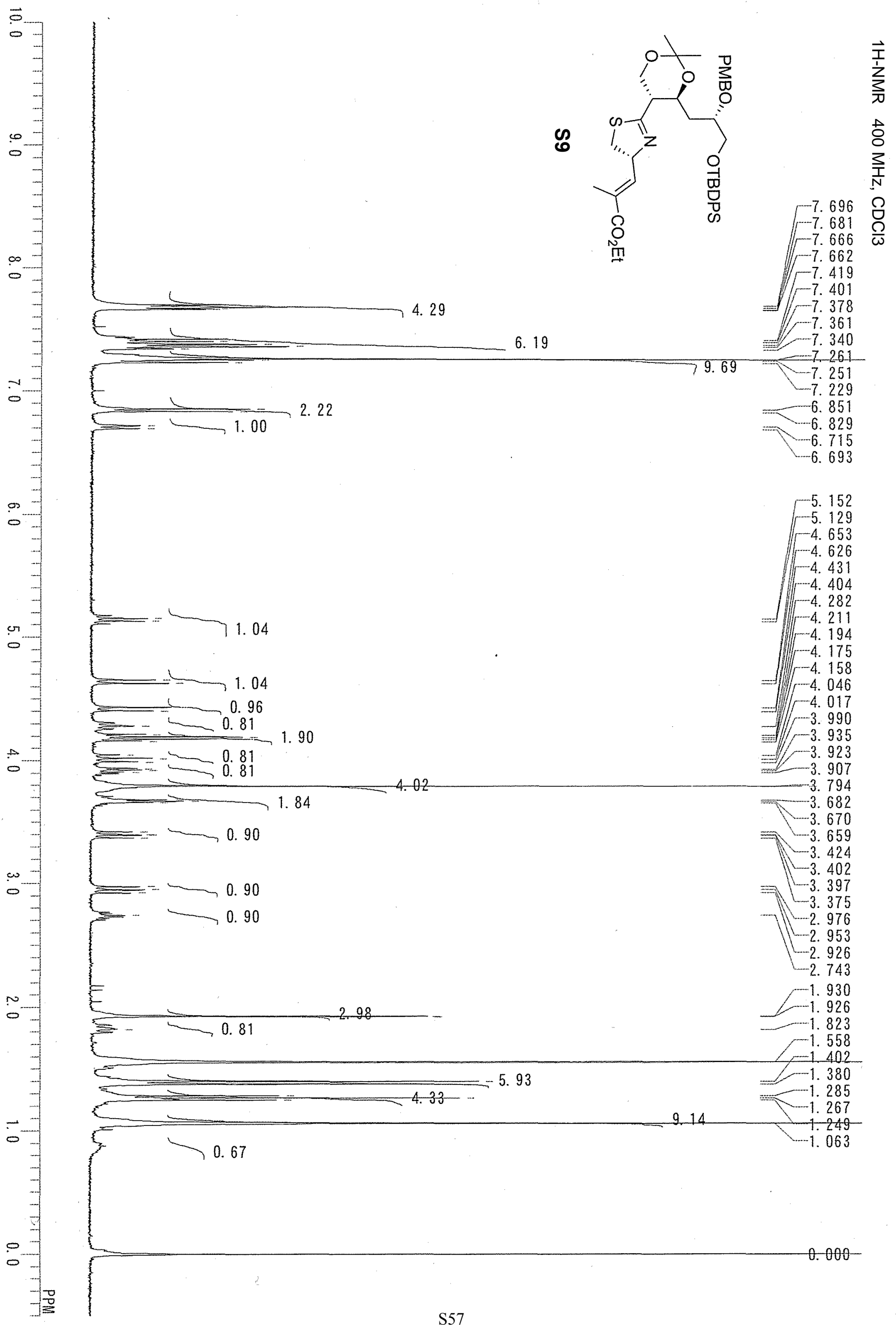



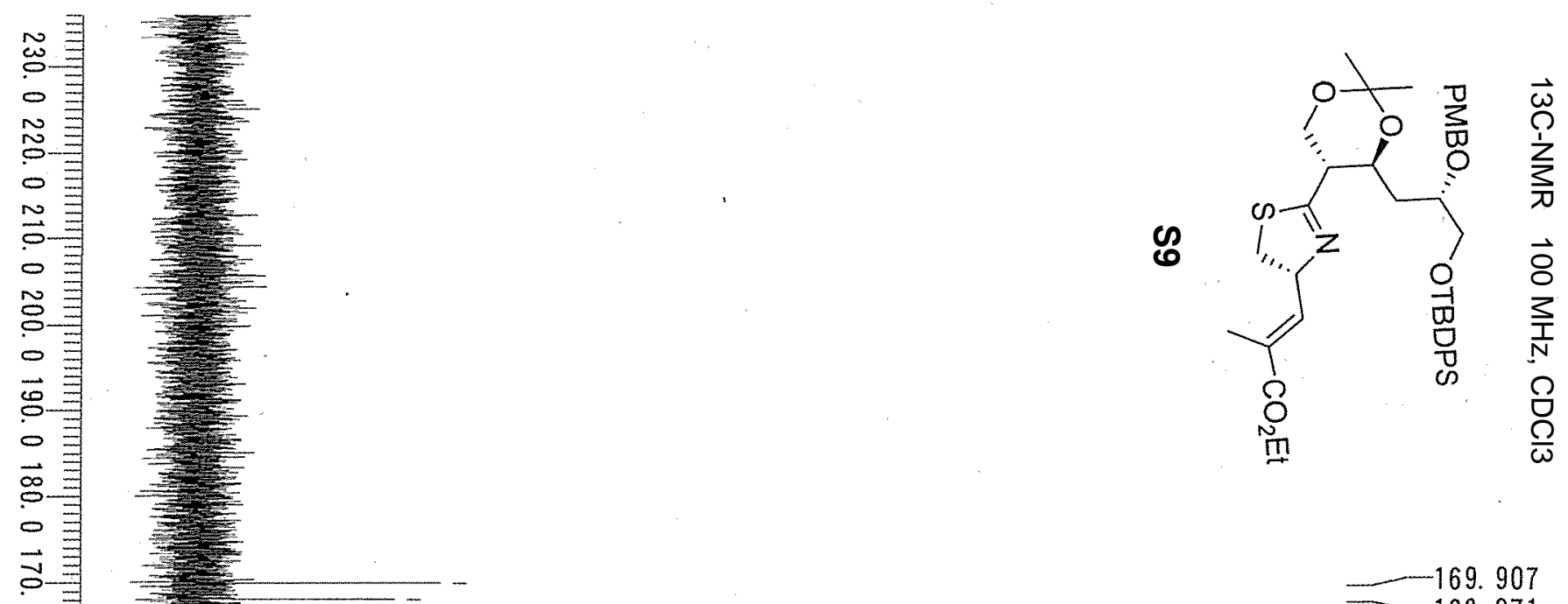

168. 071

-159. 533

용

당

$\circ$

$\overrightarrow{0}$

$\stackrel{1}{2}$

$-131.781$

$-130.505$

130. 118

$-129.607$

$-128.208$

$L_{128.158}$

$-114.196$

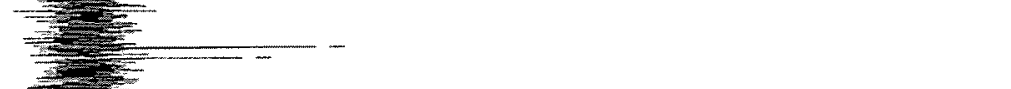

- 38.422 


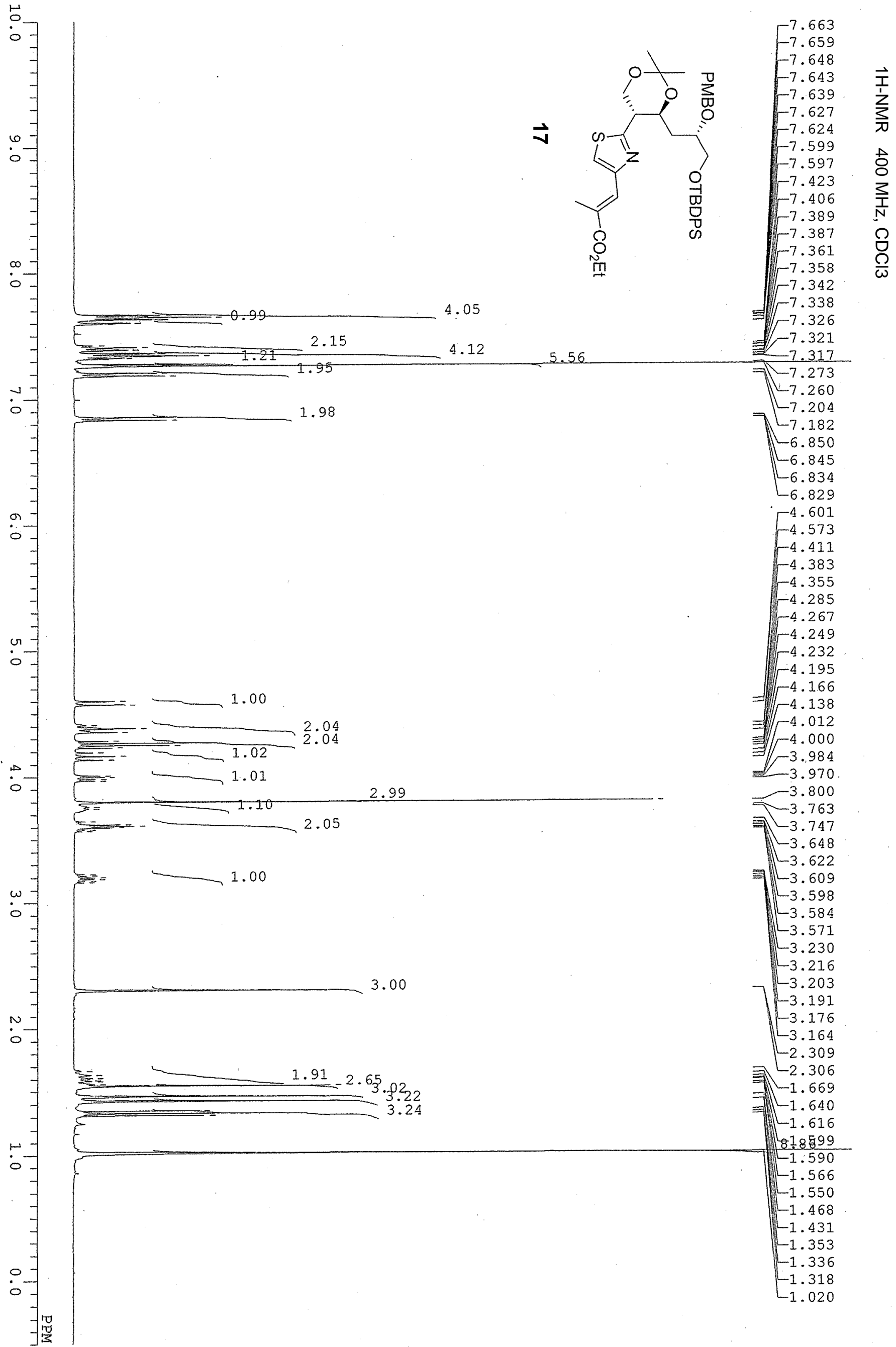




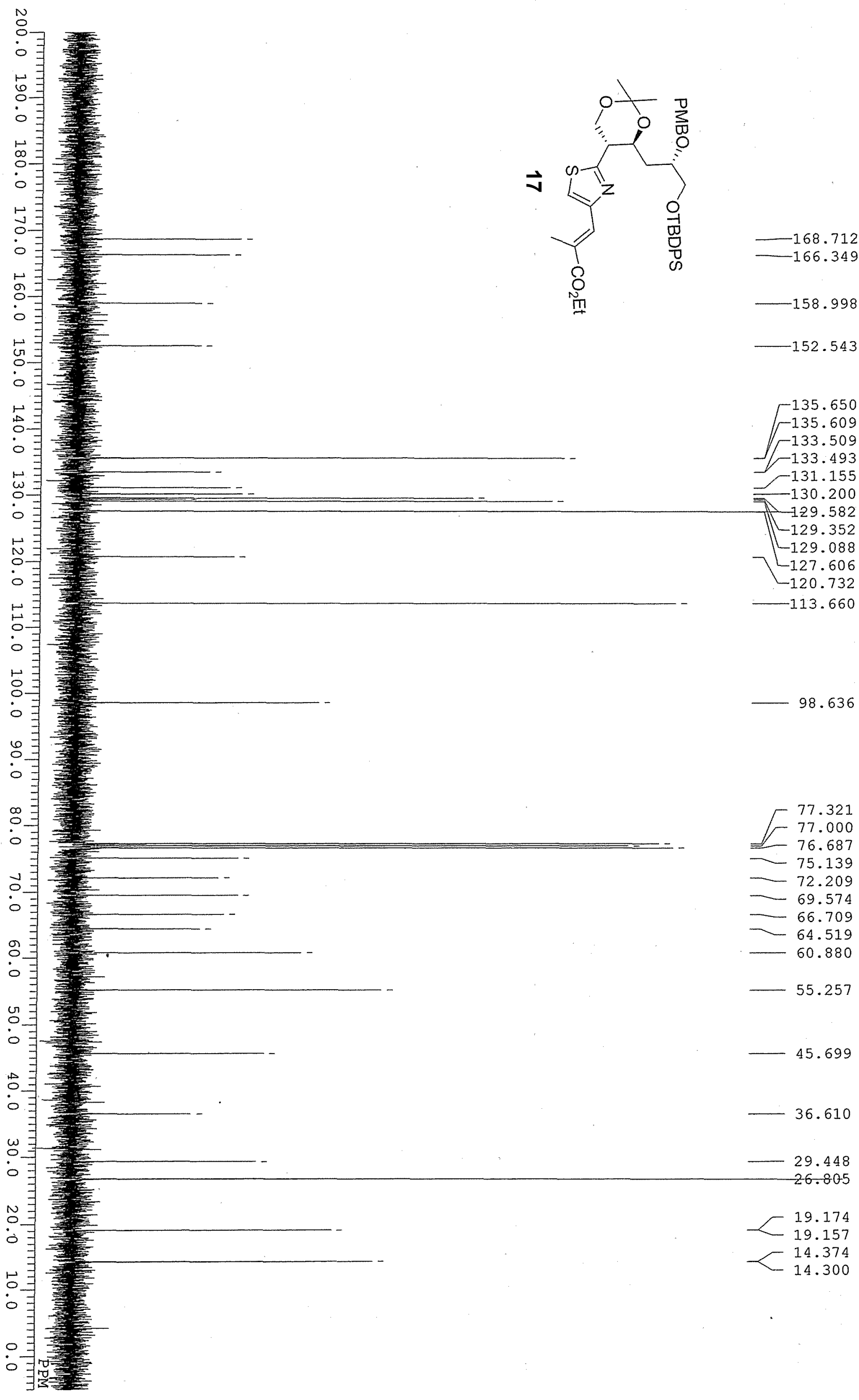




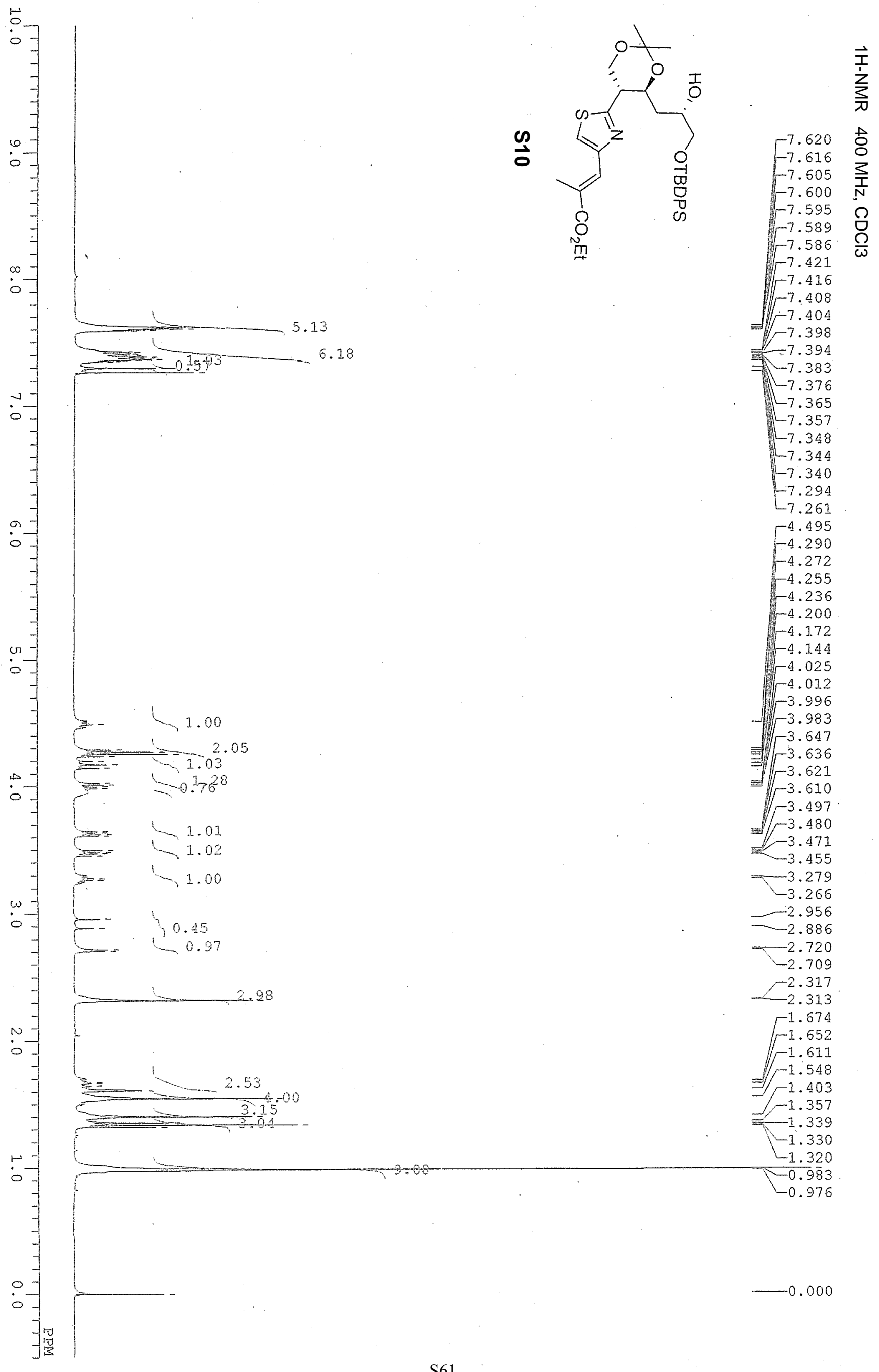




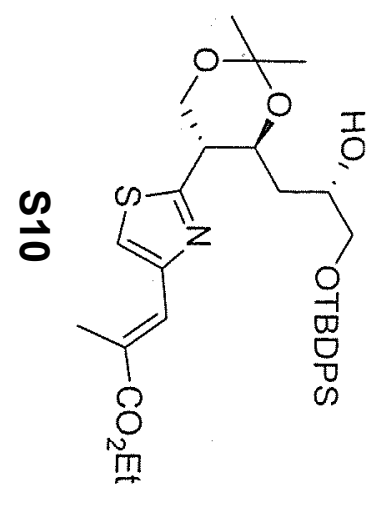

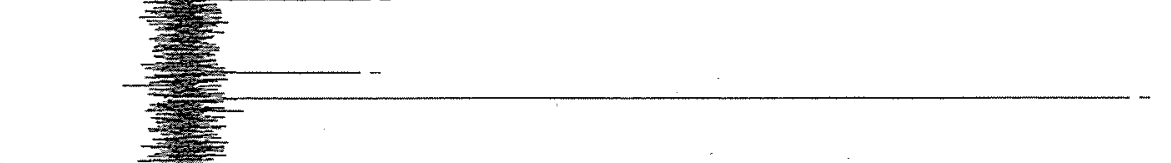

- 29.341 


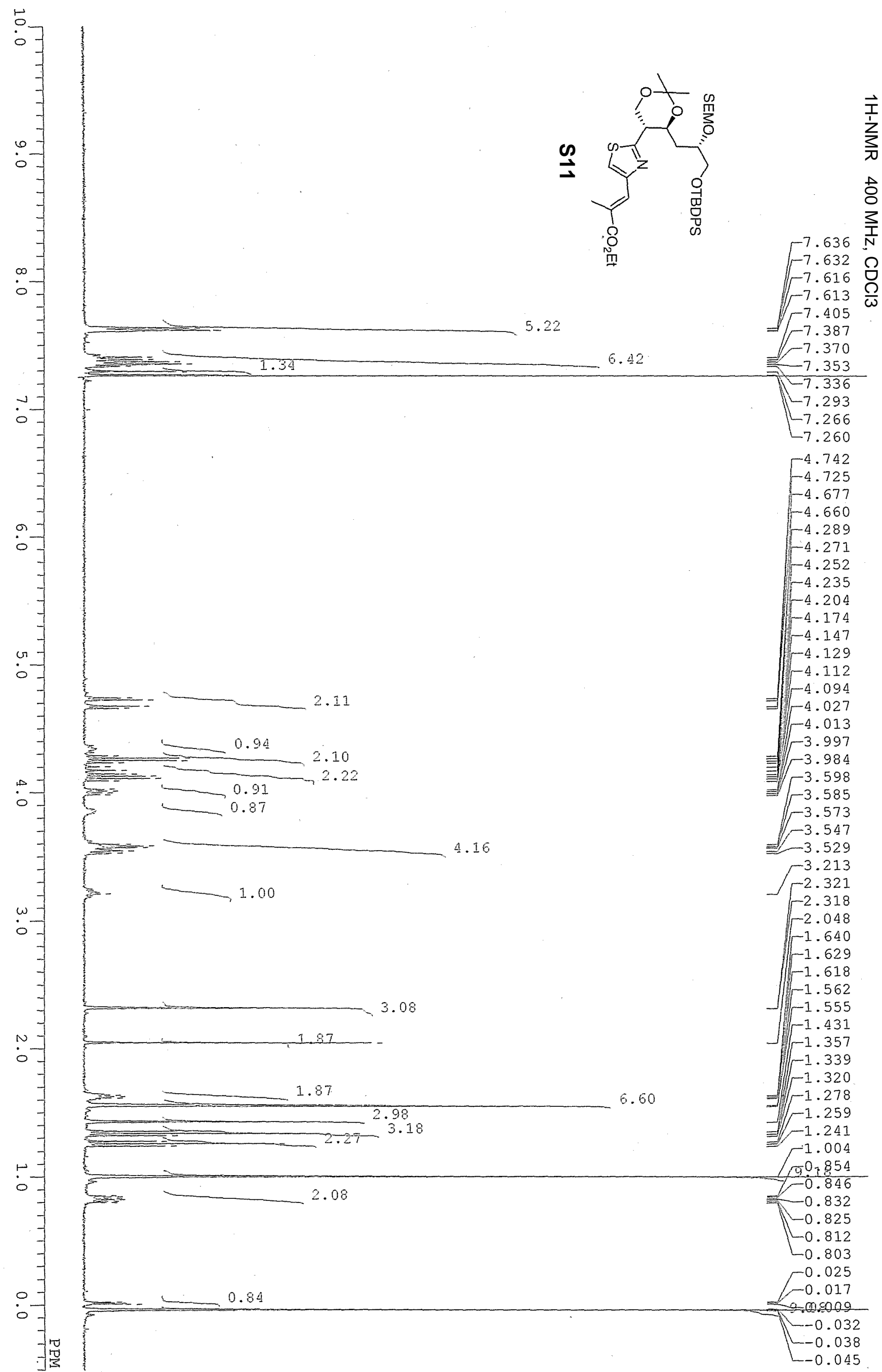



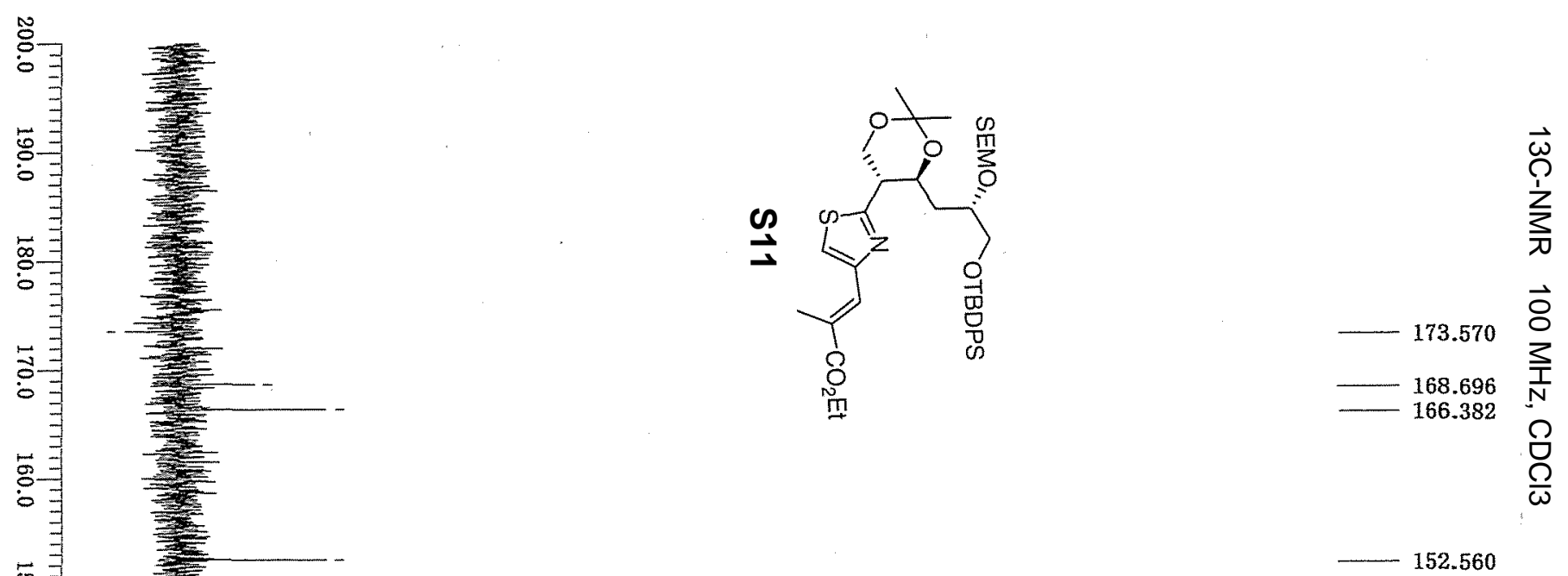

:

空

$$
\stackrel{\vec{\omega}}{\circ}
$$

옹

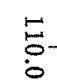
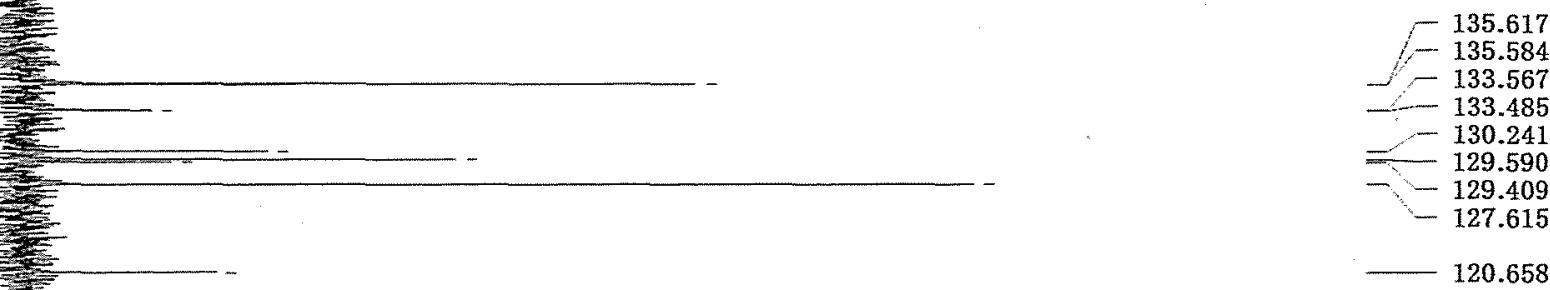

98.685

$\begin{array}{r}-\quad 95.277 \\ \hline\end{array}$

$=\quad 94.305$

91.572

$=$
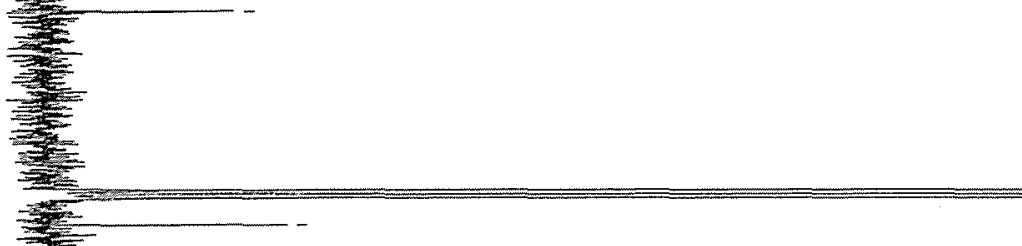

- 77.321 77.000 76.687

74.555

69.615

66.940

$-65.655$

$=65.301$

$-64.947$

$-64.511$

60.897

45.749

36.767

29.423

26.813

- 19.239

$-19.206$

$=-18.095$

18.013

14.407
$-\quad 14.316$

14.407
$-\quad 14.316$

0

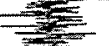

$\dot{\circ}$

-

$-1.425$

$-1.441$

提控 


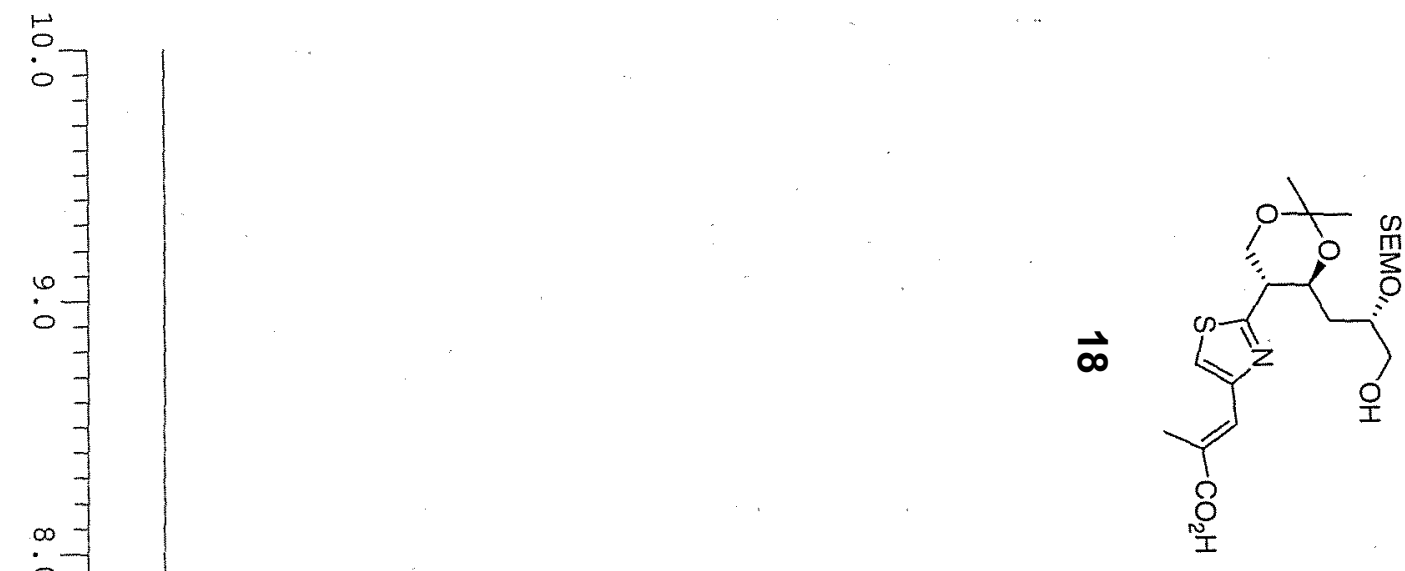

1.00

0.98

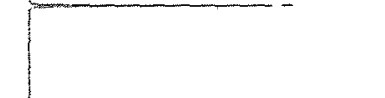

3.05

-4.777
-4.759

$-4.629$

$-4.611$

$-4.402$

$-4.378$

$-4.357$

$-4.179$

$-4.150$

$-4.123$

$-4.029$

$-4.017$

$-4.000$

!

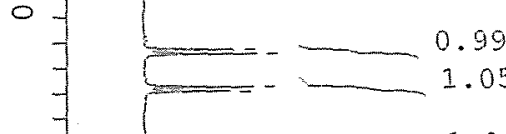

$-3.987$

$-3.787$

.05

$=-3.773$

$-3.762$

$-3.746$

$\equiv-3.739$

$-3.721$

$=-3.571$

$=-3.557$

$[-3.540$

$\equiv-3.532$

$-2.22$

$-3.514$

$\equiv-3.490$

$-3.446$

$L_{-3.429}$

$-3.416$

$-3.398$

$-3.248$

$-3.235$

-3. 222

$-2.351$

$-1.718$

$-1.687$

$-1.663$

$-1.562$

$=-1.504$

3.29

-1.504
$=1.447$

$\Gamma^{0.957}$

$\sqrt{-0.941}$

:

2.16

$-0.930$

$-0.920$

$-0.914$

$-0.904$

$:$

5 


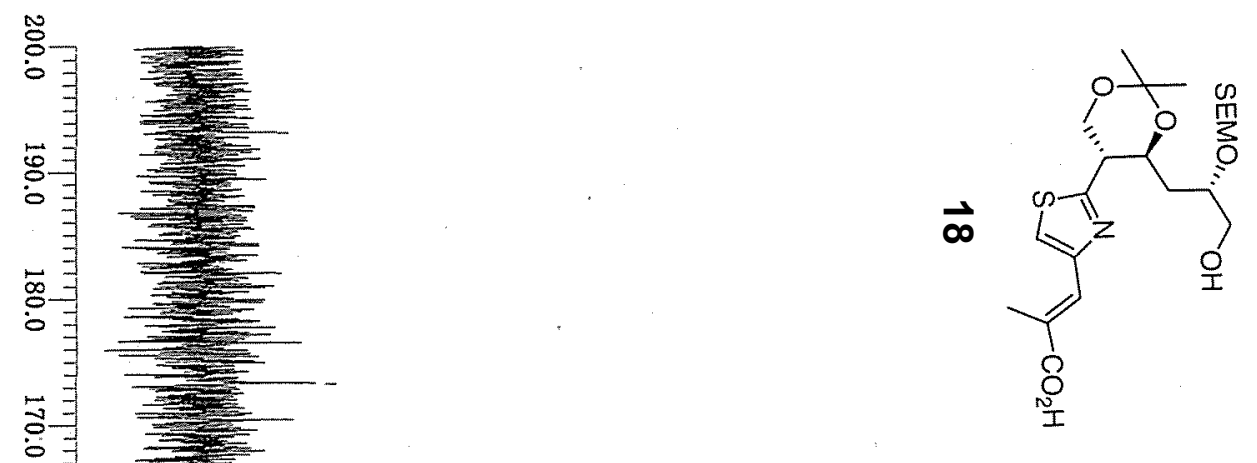

\section{8}

- 69.393

- 66.174 

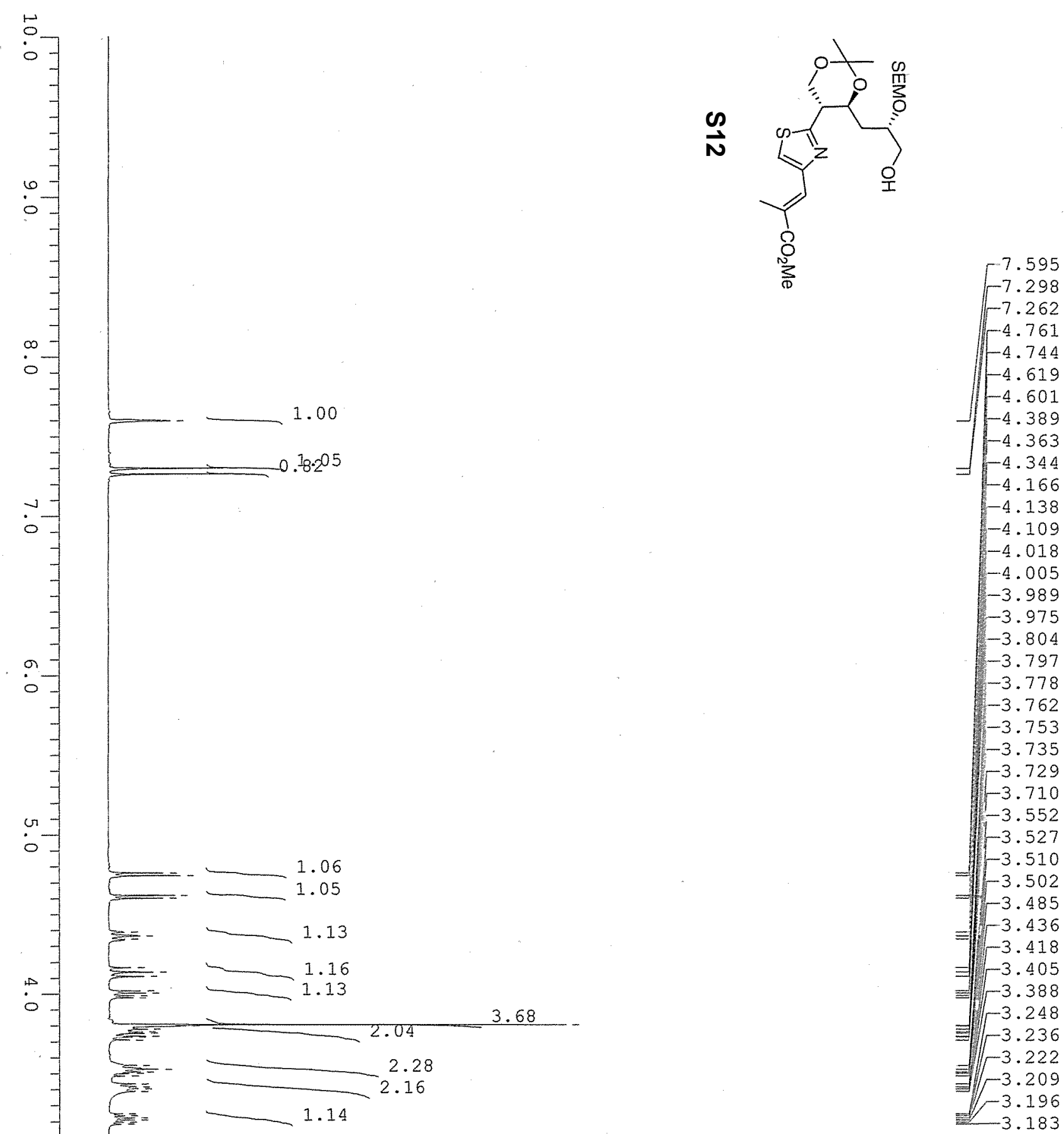

$-2.337$

$-2.333$

$-2.032$

$-1.712$

$-1.681$

3.33

$N$

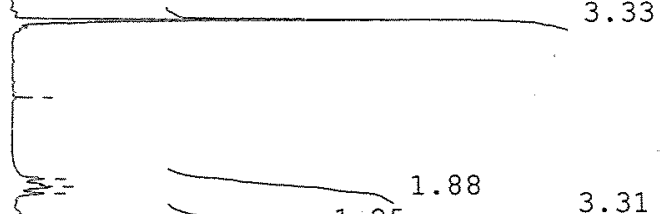

$-1.652$

$-1.554$

] -1.531

$-1.523$

1.507

$\exists /-1.497$

$\equiv-1.471$

3.70

$-1.441$

$-1.248$

$-0.952$

$-0.943$

$-0.936$

$-0.926$

$L_{0} .916$

Lo.909

$-0.900$

:-

$-0.015$

10.800

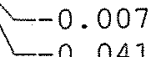




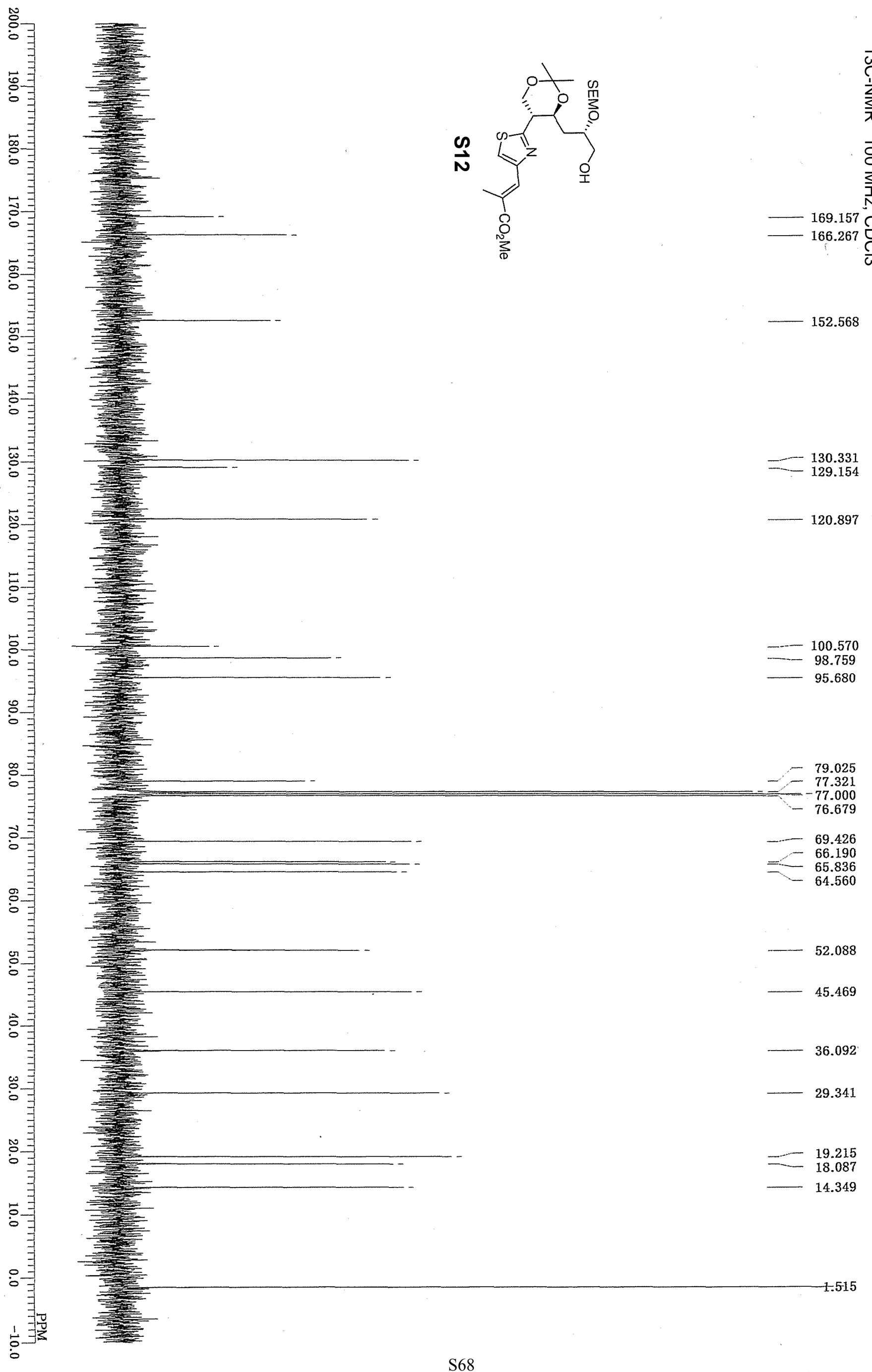



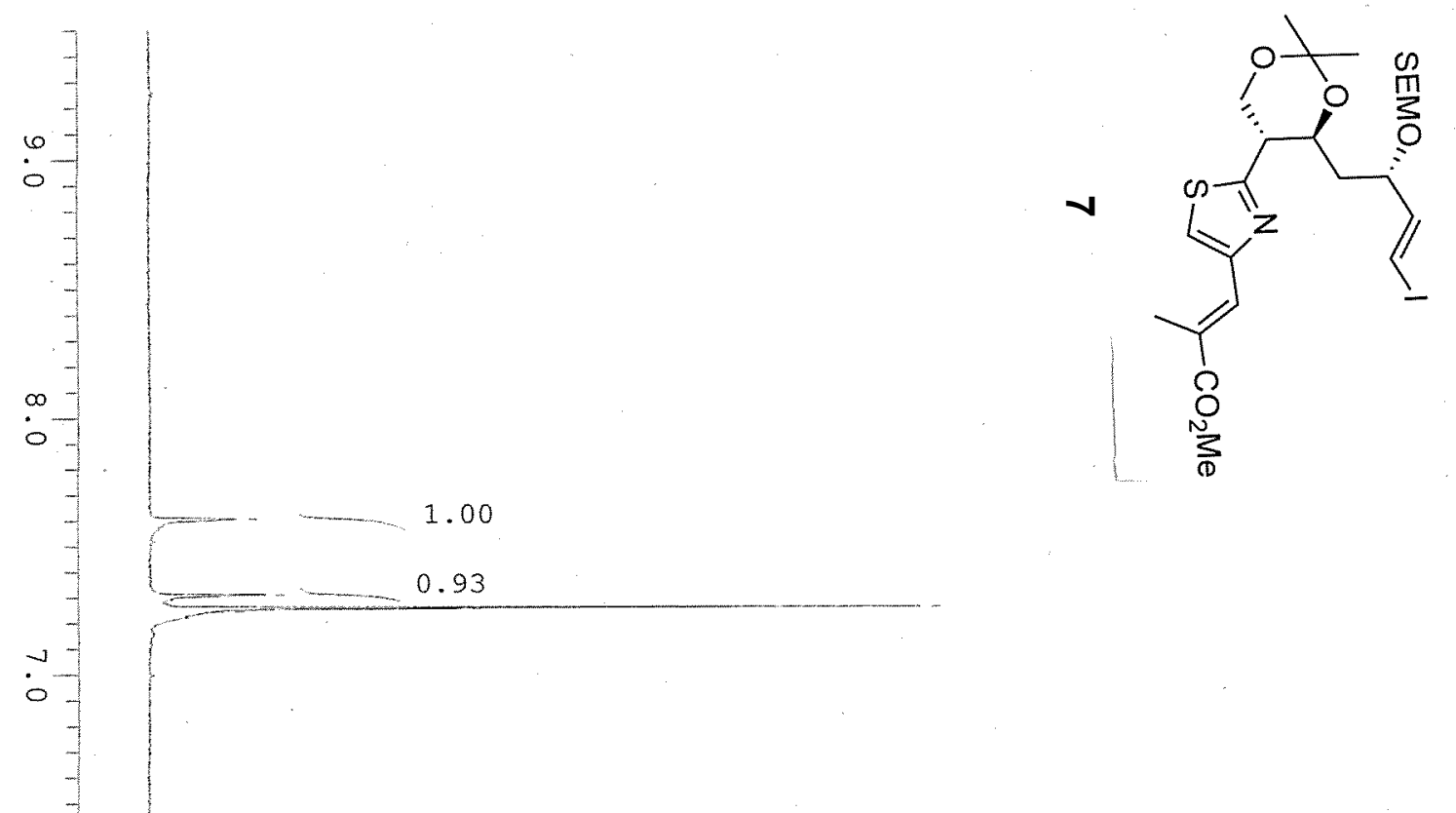

$-7.603$

$-7.308$

$-7.260$

$-7.253$

$-7.242$

. -6.412

- -6.394

$-6.375$

$-6.357$

$-6.322$

$-6.316$

$1-6.285$

II) -4.656

$4-4.639$

-4.593
-4.574

$-4.574$

$-4.399$

$-4.374$

$-4.350$

$-4.215$

$-4.196$

$-4.169$

4.140

$-4.112$

$-4.019$

$-4.006$

3.990

3.977

$-3.814$

-3.808
-3.651

- 3.651

113.628

J) 3.606

3.581

1. -3.558

$-3.539$

$-3.532$

1赫 -3.515

$-3.240$

$-3.227$

$-3.214$

$-3.201$

$-3.187$

$-3.175$

3.17

$-2.344$

$-1.740$

$-1.705$

$-1.680$

- -1.622

111.6913

$-1.558$

$-1.451$

$--1.251$

0.925

0.925
0.904
0.899
0.882

2.76

4

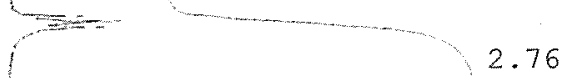

$-0.027$

$-0.018$

$-0.012$

-0.002
-0.008

-0.008
-0.021 


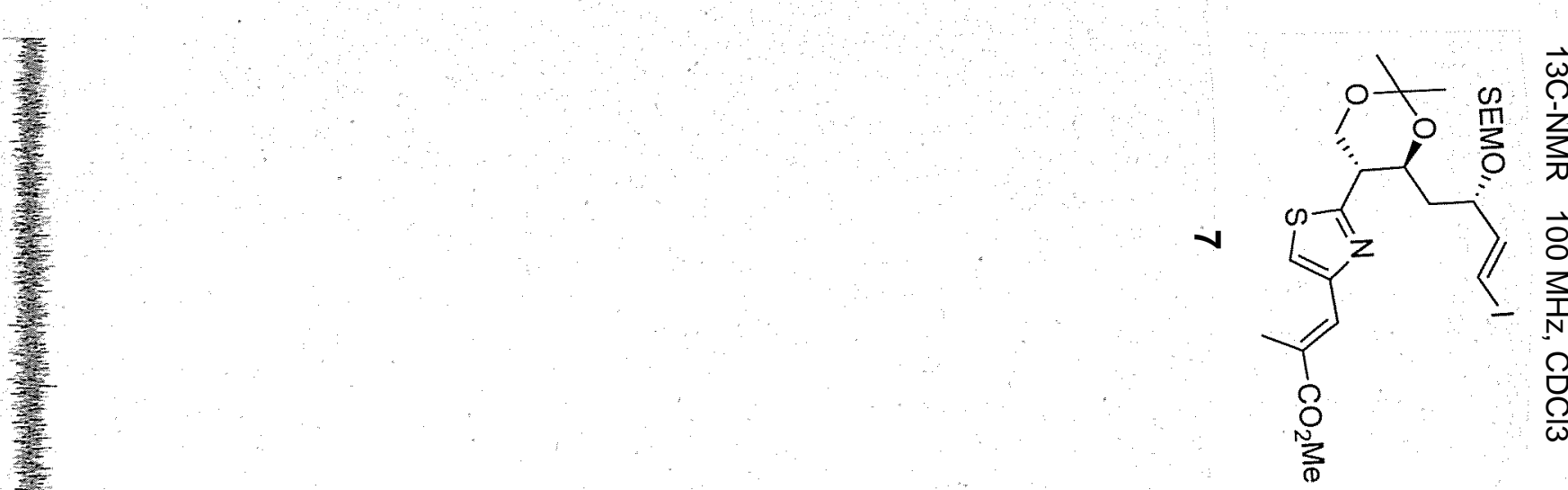

$-169.190$

$-166.160$

o.

o

G

○

$\stackrel{1}{\circ}$

o

$\stackrel{\leftarrow}{\omega}$

年

菏

$\circ$

苫

$\circ$

客

$\circ$

$\because$

$-152.609$

$-146.575$

$=-130.348$
-129.138

$--121.004$

98.743

93.243

78.383

77.346

77.025

76.712

74.852

69.113

65.483

64.569

8

$\leqslant$

$-$

52.138

45.379

39.591

29.440

w

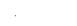

N

19.223

18.029

$-14.407$

㟧

$\circ$

$-1.359$

Ird

品包

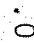




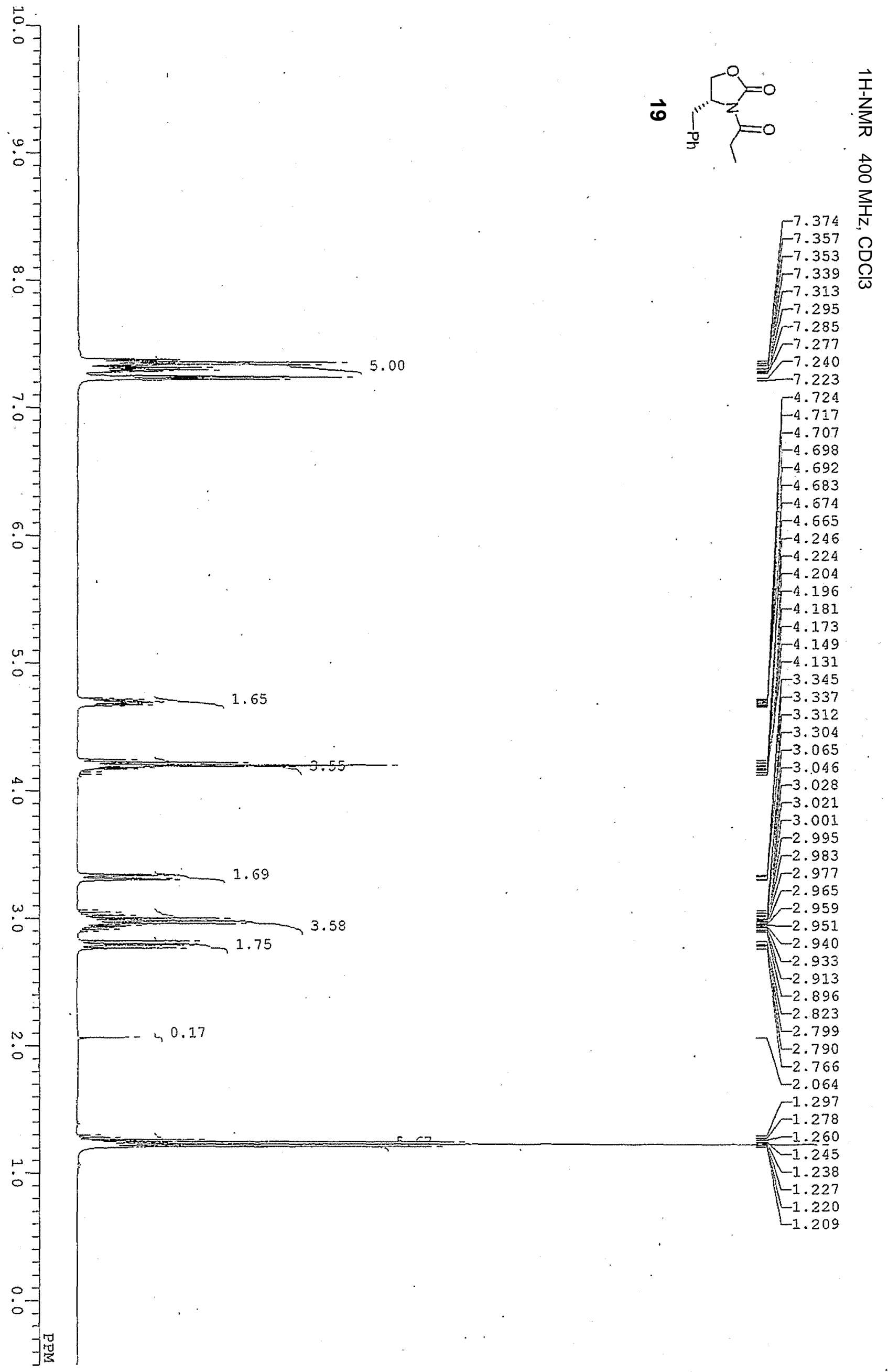




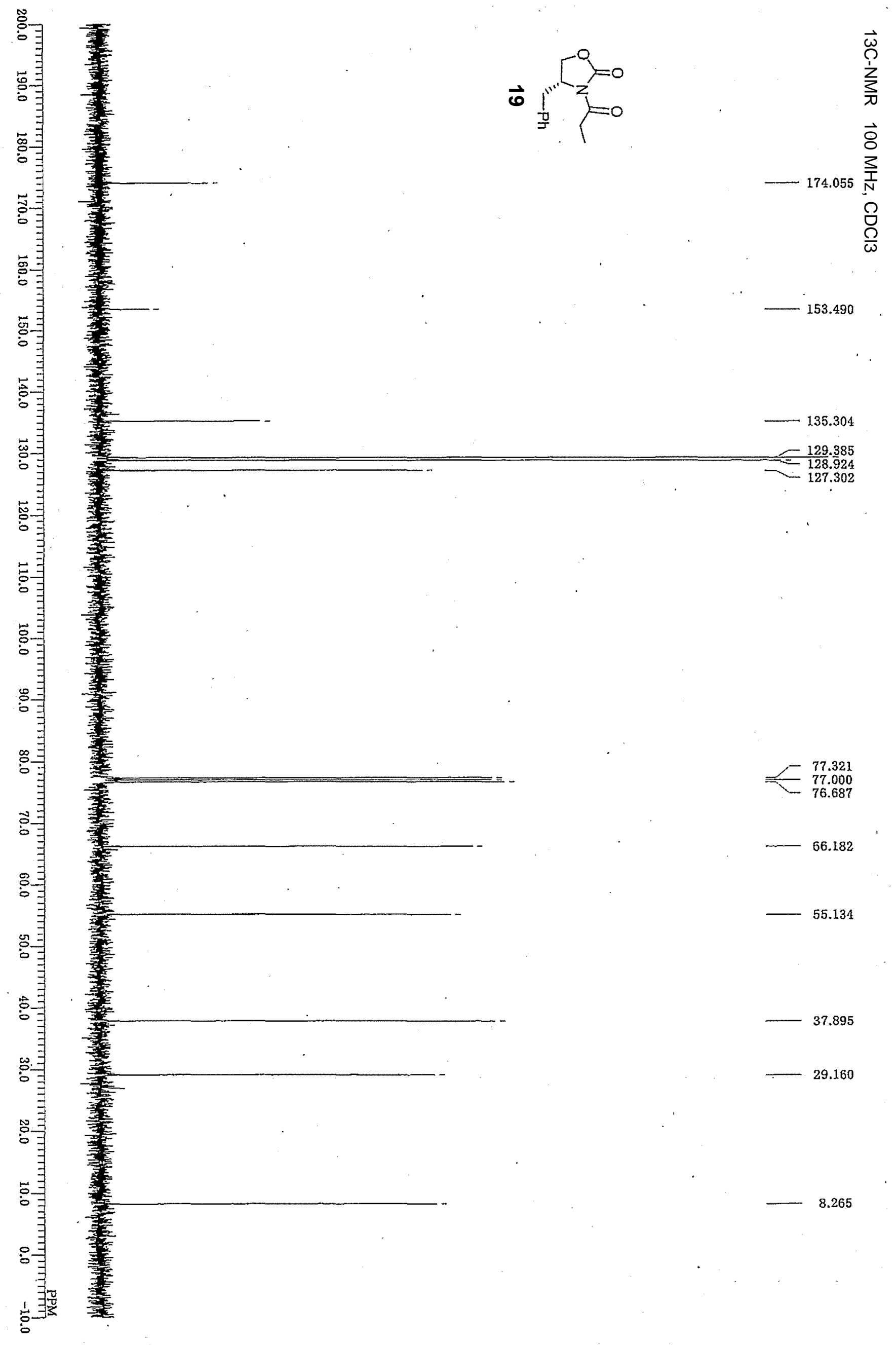




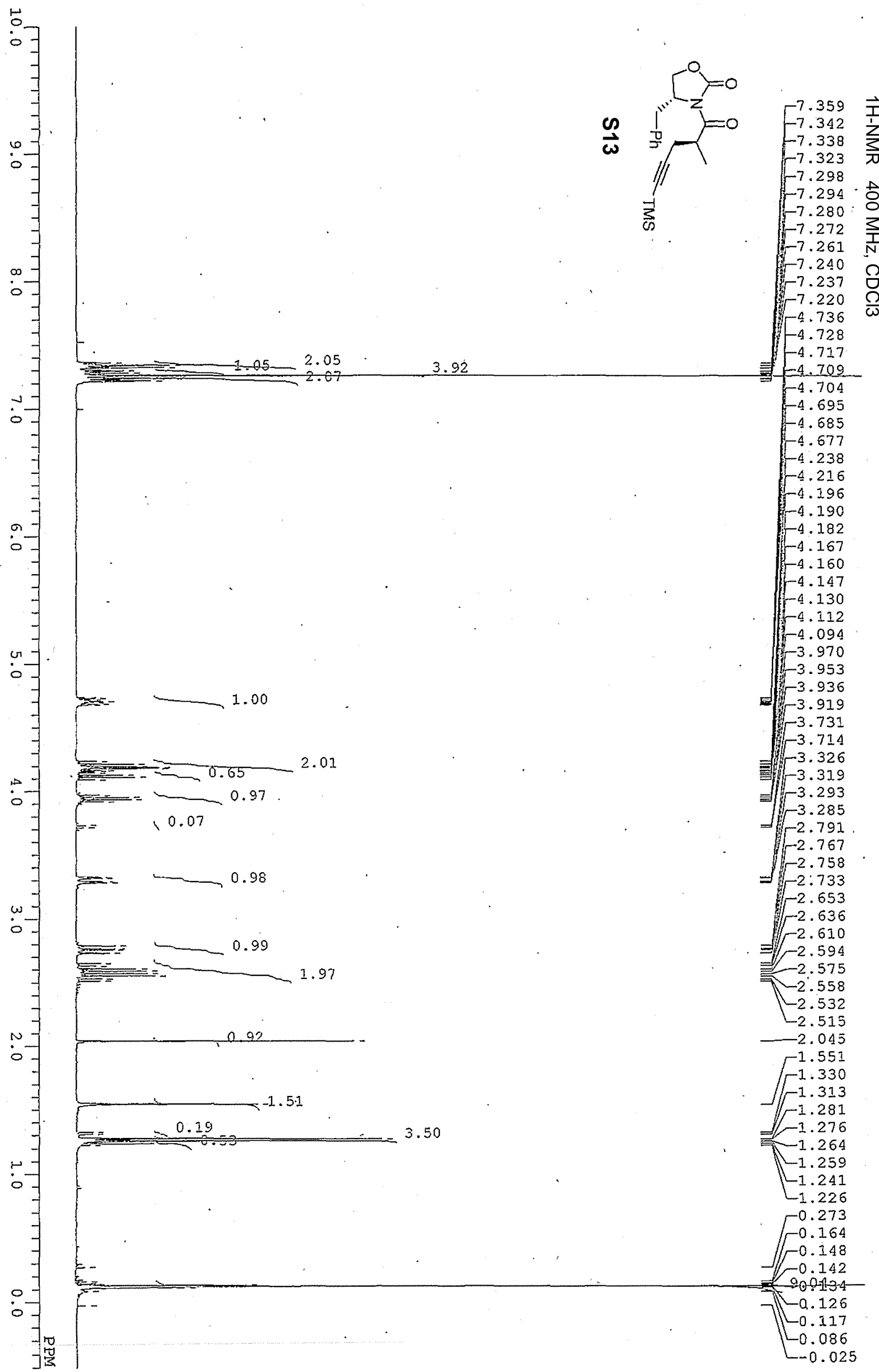




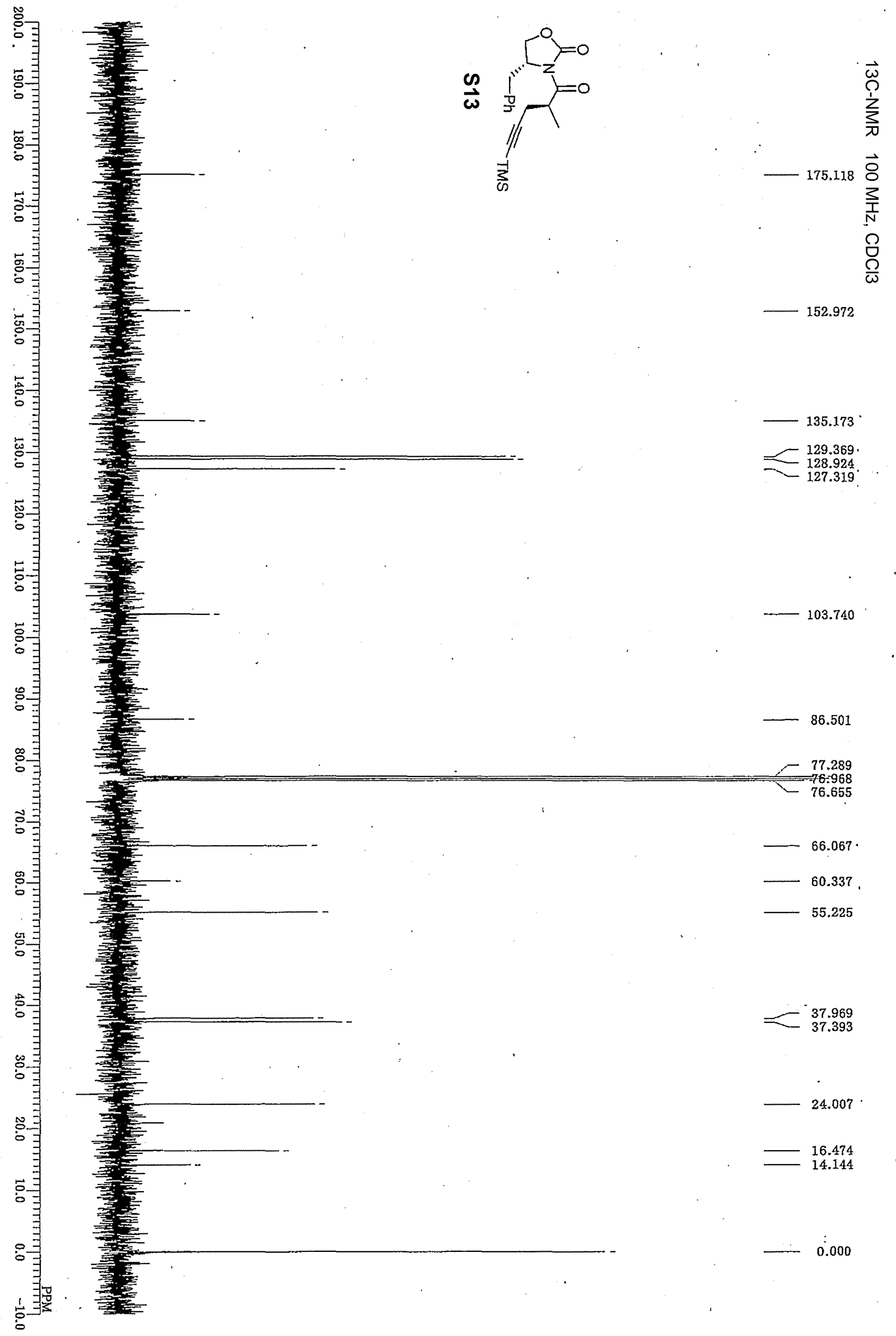




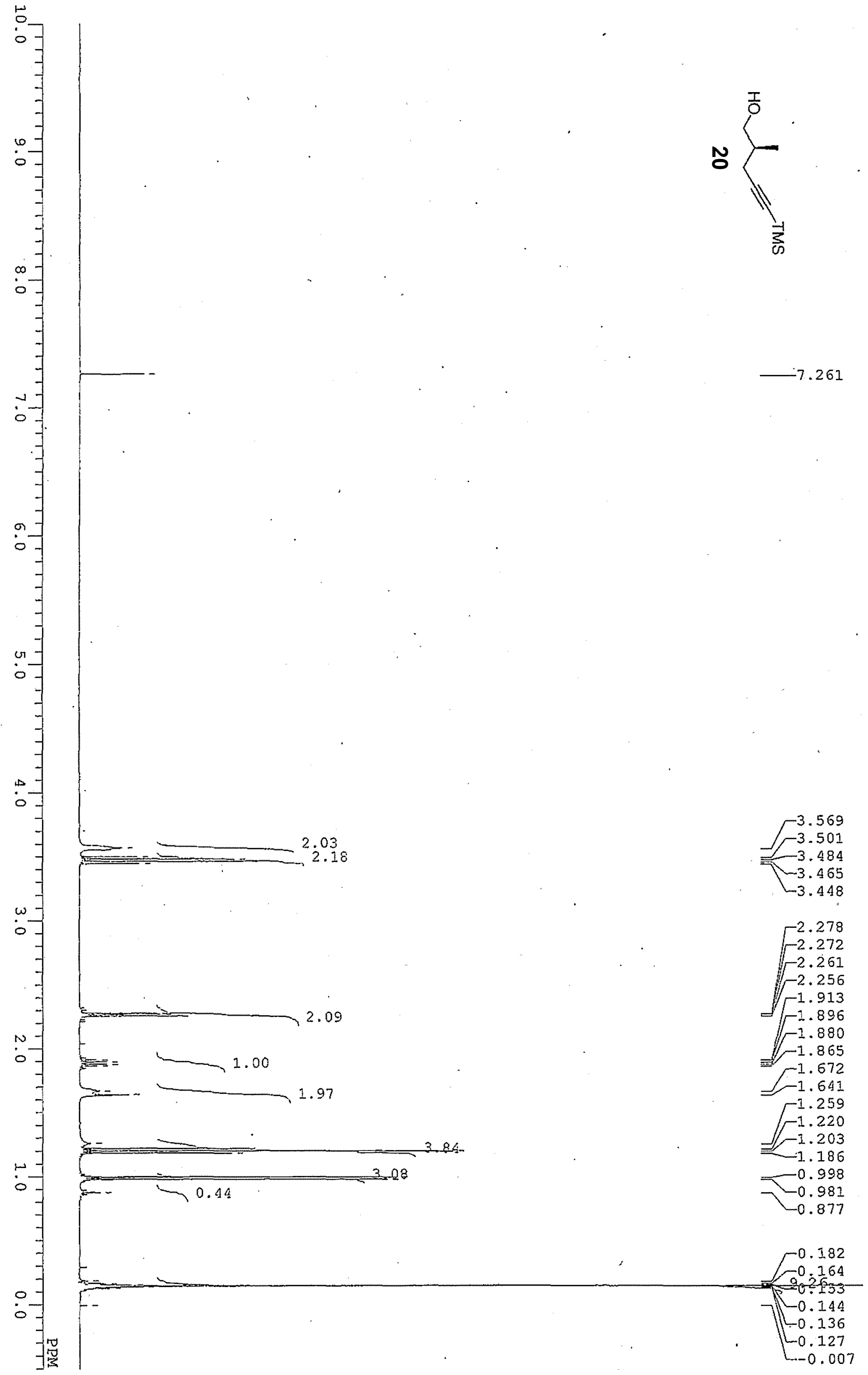




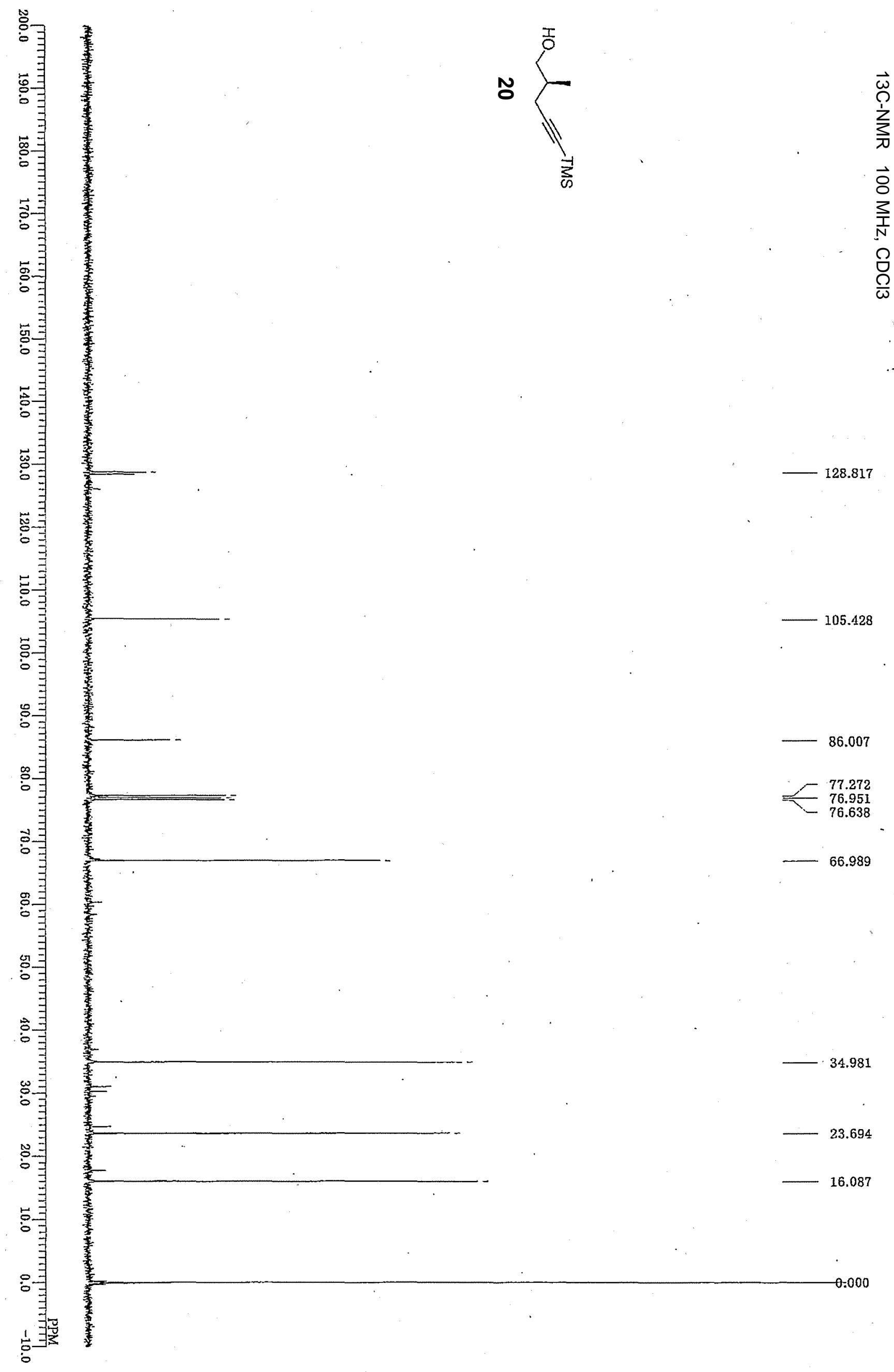




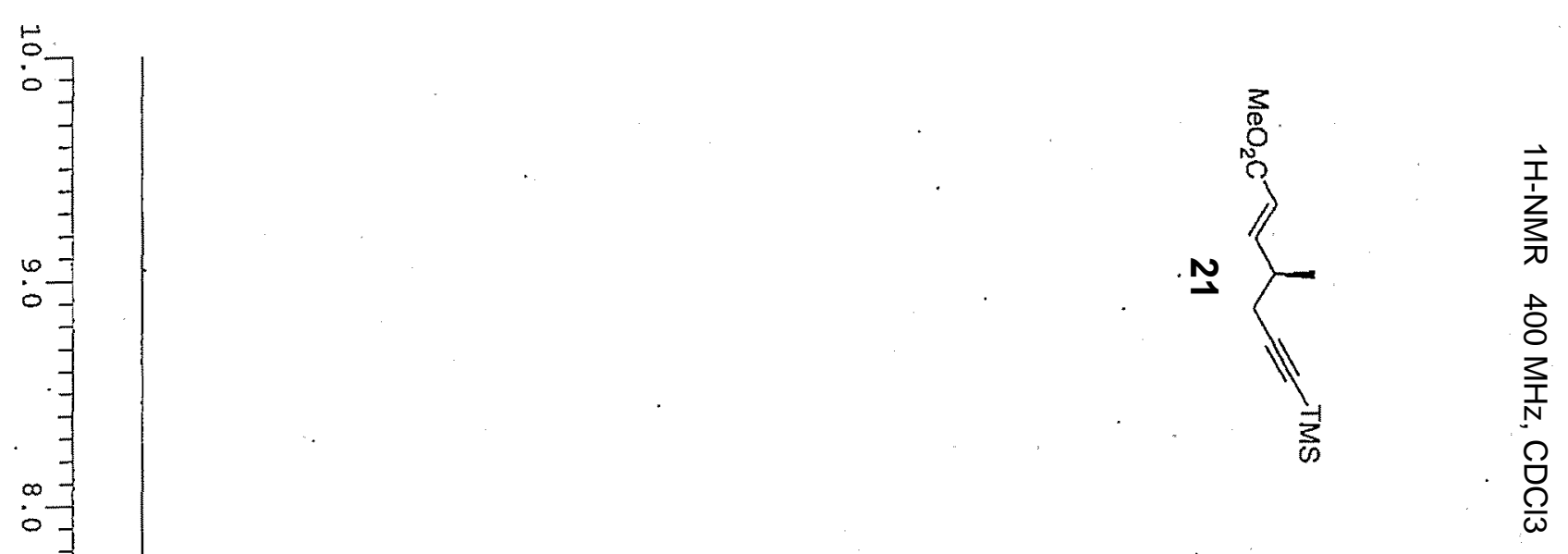

7.260

$-7.254$

6.957

$-6.939$

- 6.917

$-6.899$

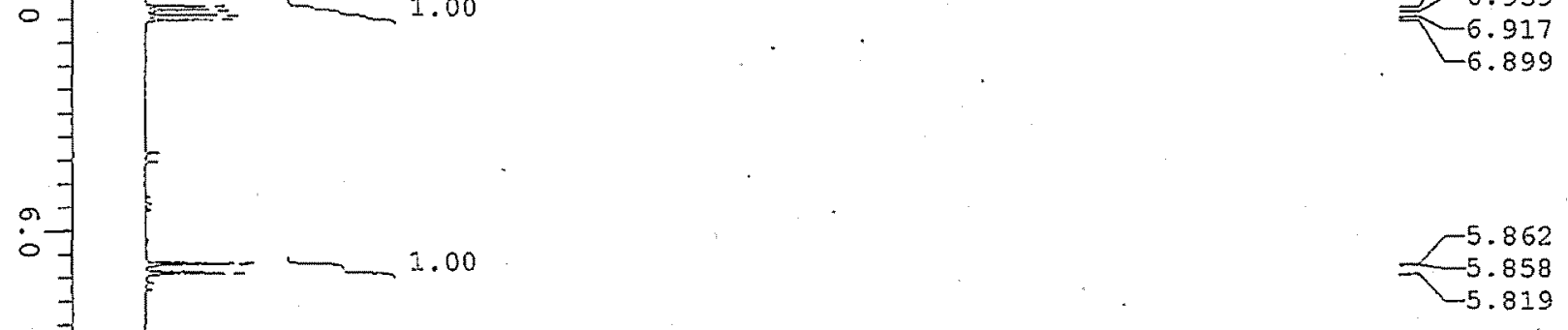

o

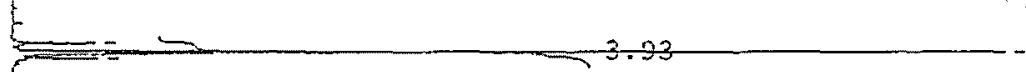

$-3.735$

$-3.728$

$-3.711$

$-3.024$

$-3.007$

$\sqsupseteq-2.562$

$-2.545$

$-2.527$

$-2.356$

$-2.340$

$1 \mathrm{y}^{-2.340}$

c

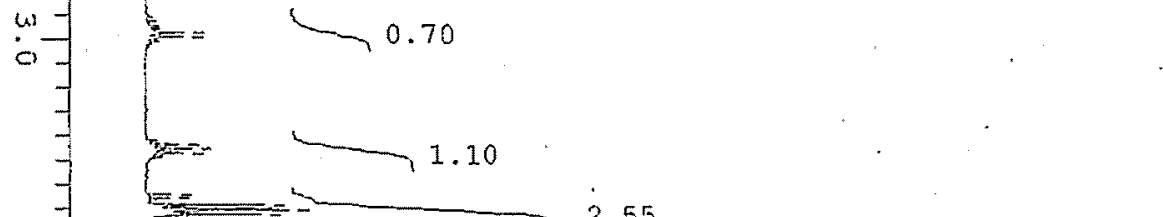

11 2.29

$-2.292$

$-2.273$

$\equiv\{-2.249$

$-2.232$

$-2.181$

:

-

2.55

$-1.586$

$-1.251$

$-1.164$

$-1.157$

$-1.147$

$-1.140$

$-1.134$

$-1.117$

$-1.106$

$-1.088$

$-1.024$

$-1.006$

$-0.988$

$-0.151$

:-1

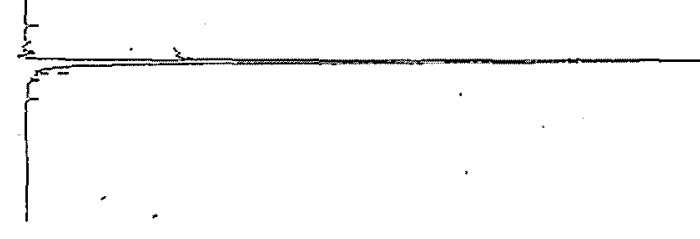

10.253

$-0.136$

$-0.094$ 


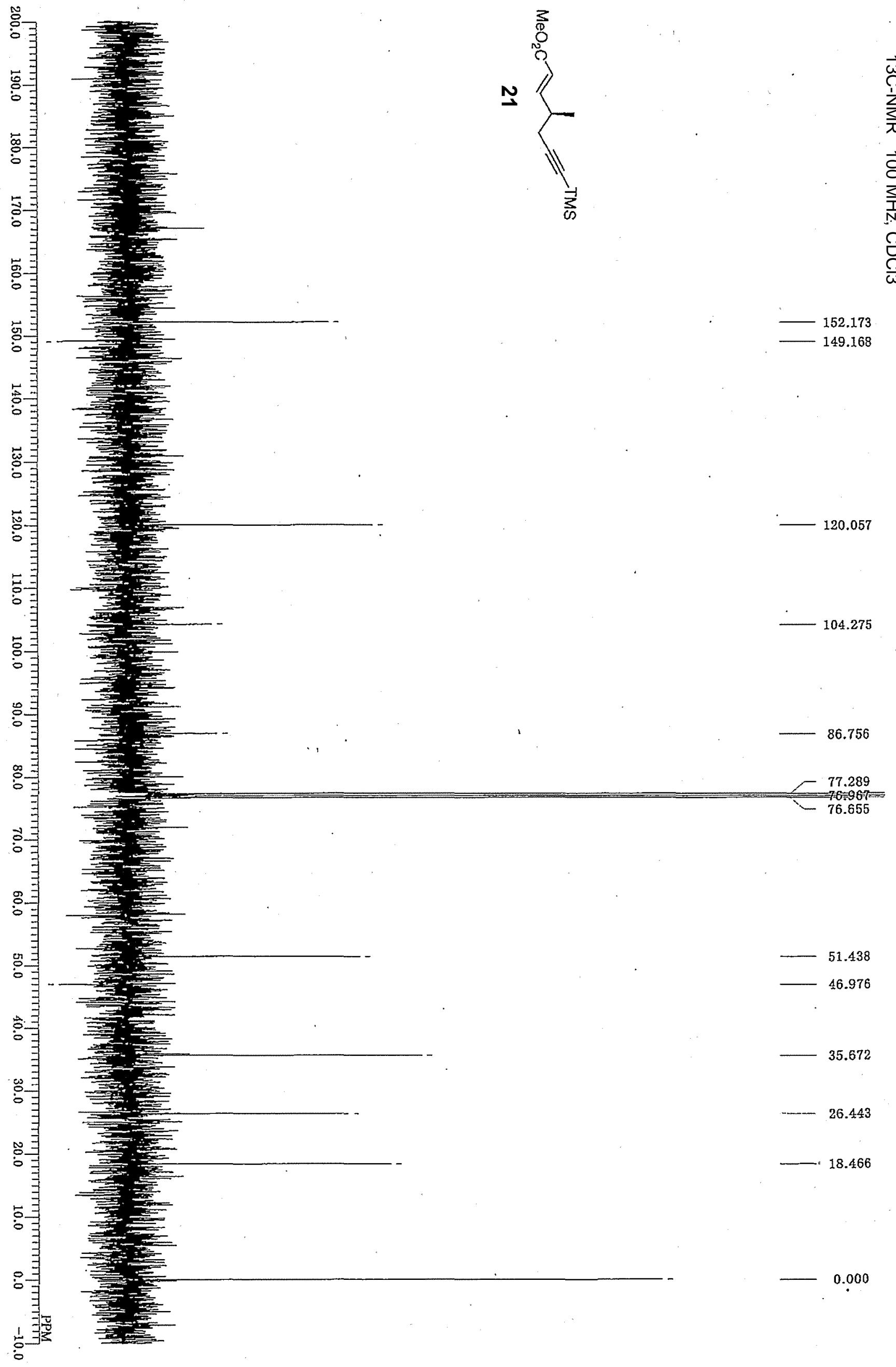




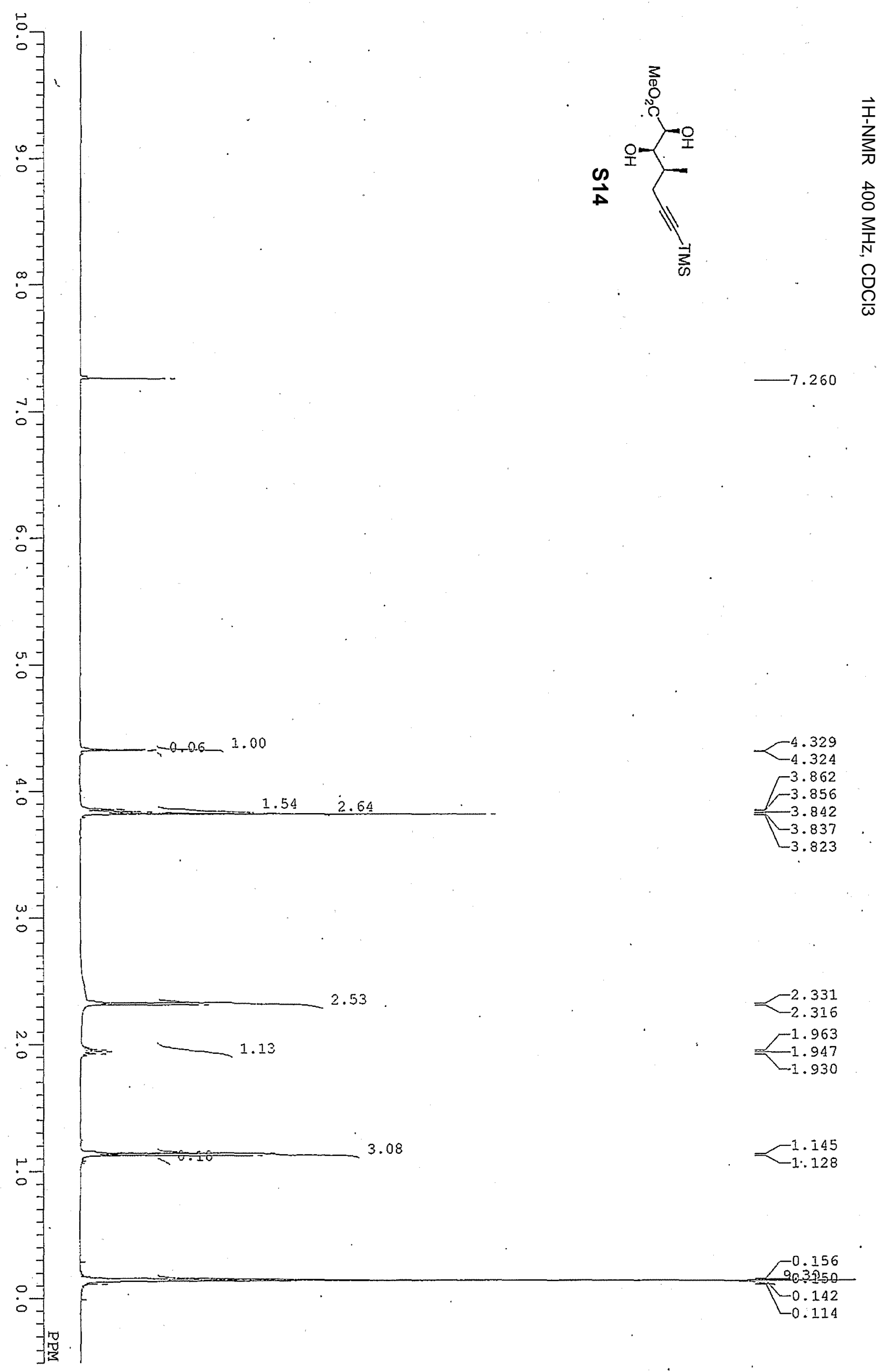



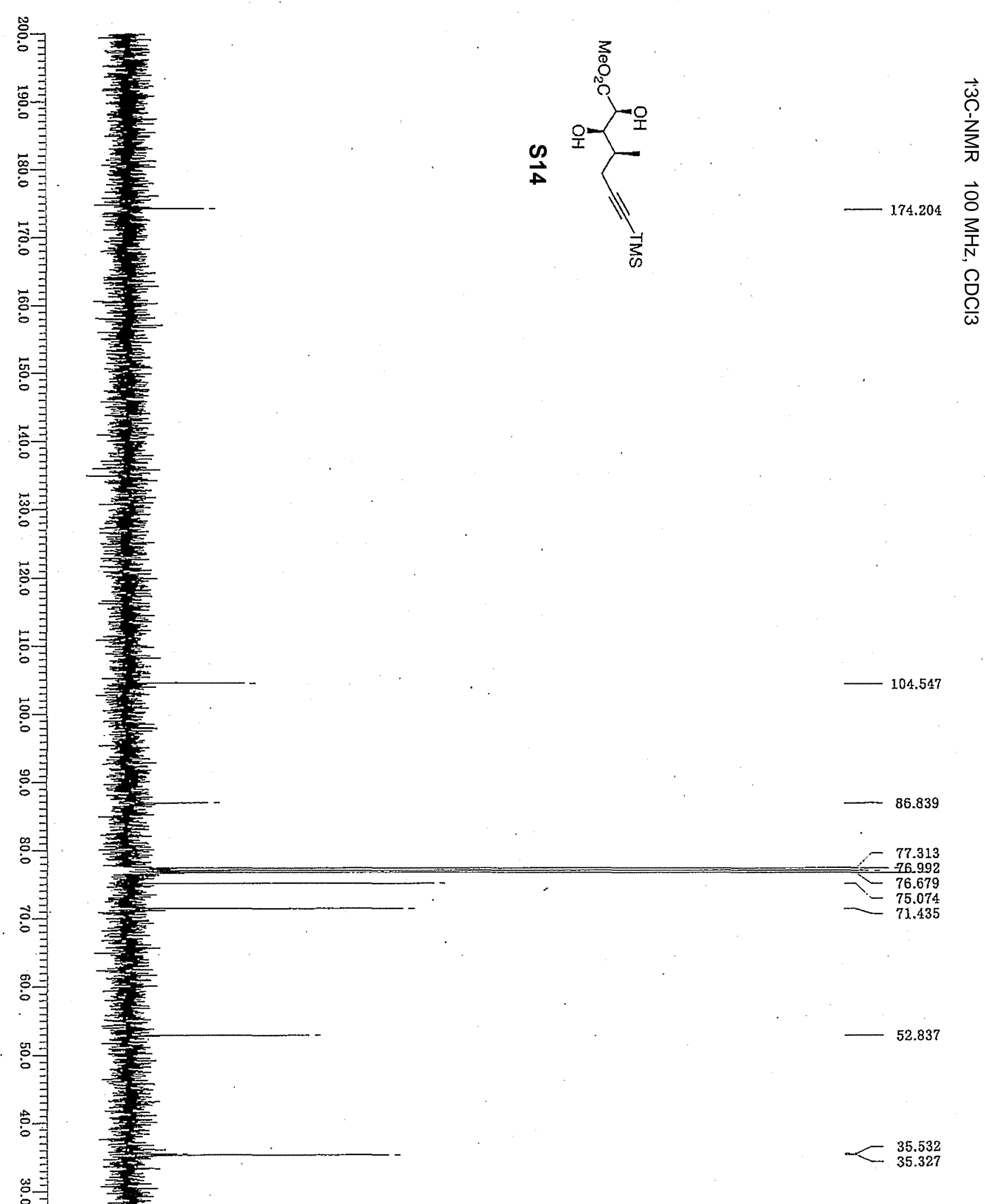


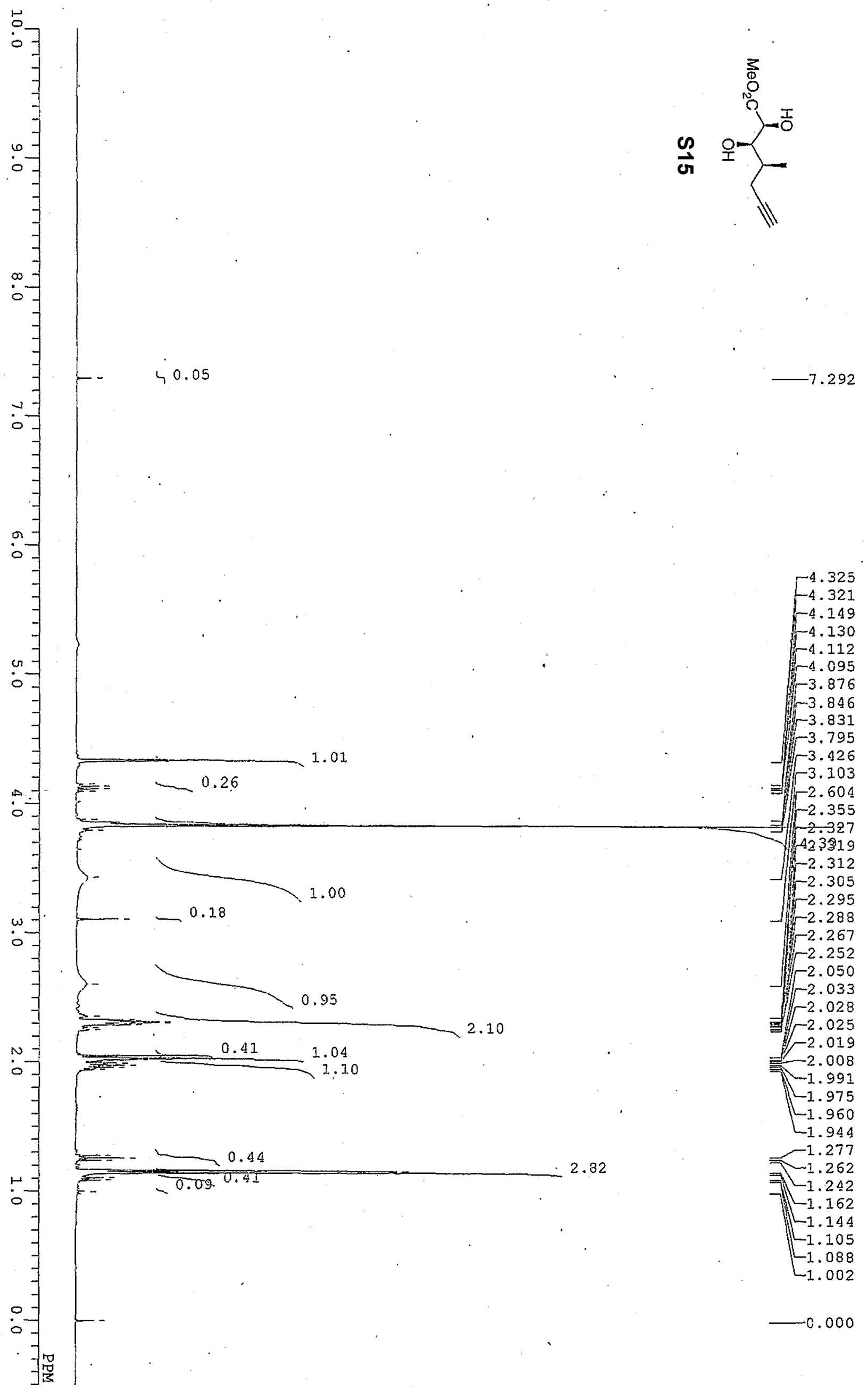

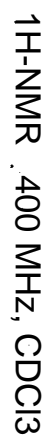

$-4.325$

$-4.149$

$-4.130$

$-4.112$

095

$-3.846$

$-3.831$

$-3.795$

$-3.103$

$-2.604$

$-2.355$

423319

$-2.312$

$-2.305$

$-2.295$

$-2.252$

$-2.050$

${ }^{-2.033}$

$-2.025$

$-2.019$

$-2.008$

$-1.991$

$-1.975$

$-1.944$

$-1.277$

$-1.242$

$-1.162$

$-1.088$

1.002 


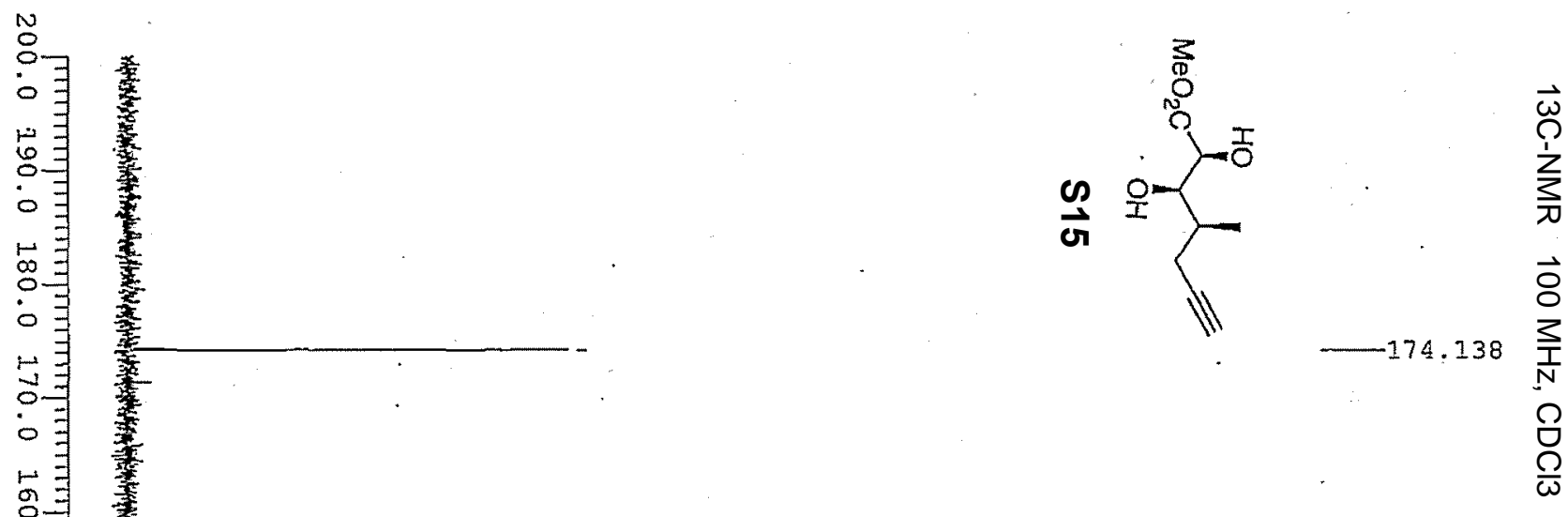

范

:

웅

$\omega$

○

怘

$\circ$

。

。

0

$\because$

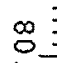

○

ㅇ..ㄱ..

手

용크

능

烈

品

.

$\omega$

크

읔

吕寻

$\circ \exists$

$\circ \exists$

寻

岁灌

:

81.824

$\tau 77.321$

77.000
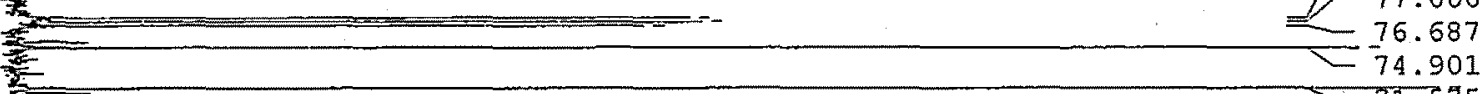

琴

$-71.575$

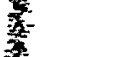

在

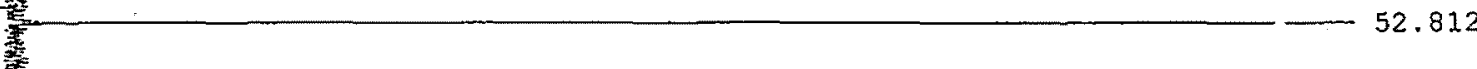

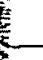

蓄

34.956

青变

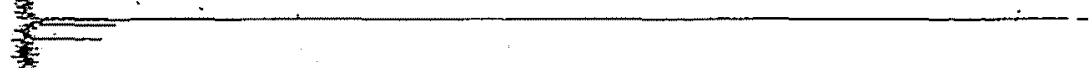

$-22.557$

15.296

箞 


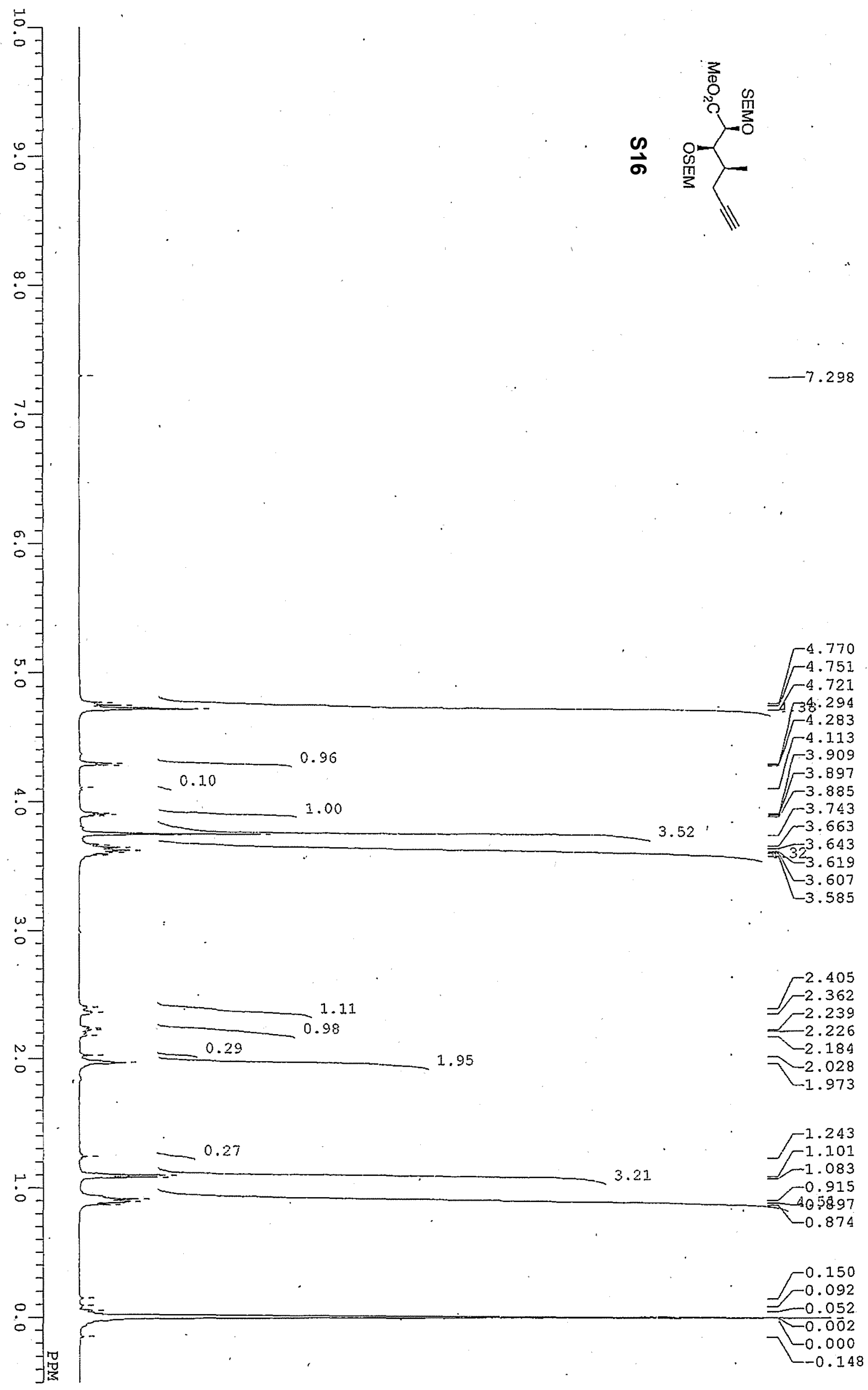




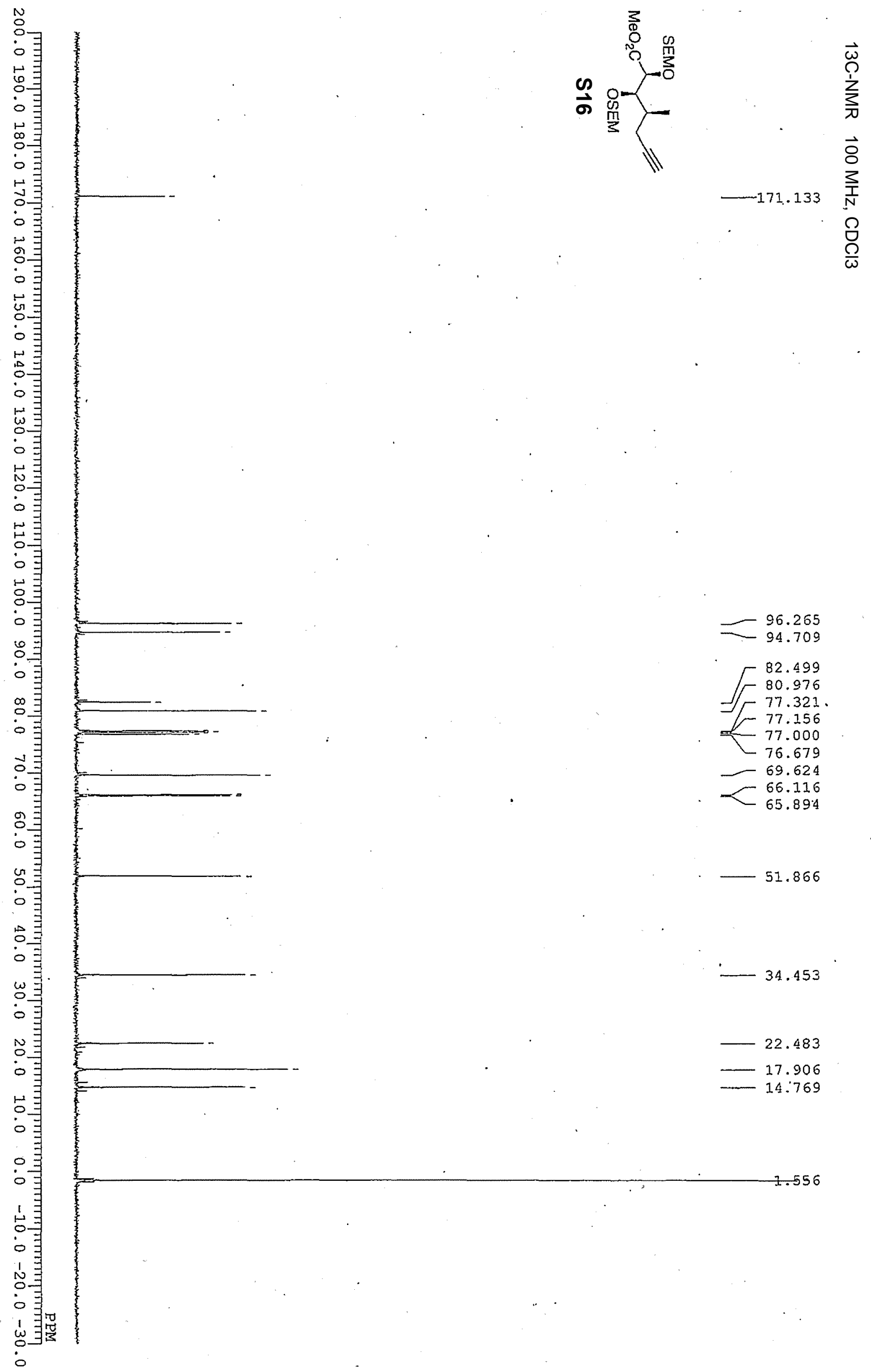




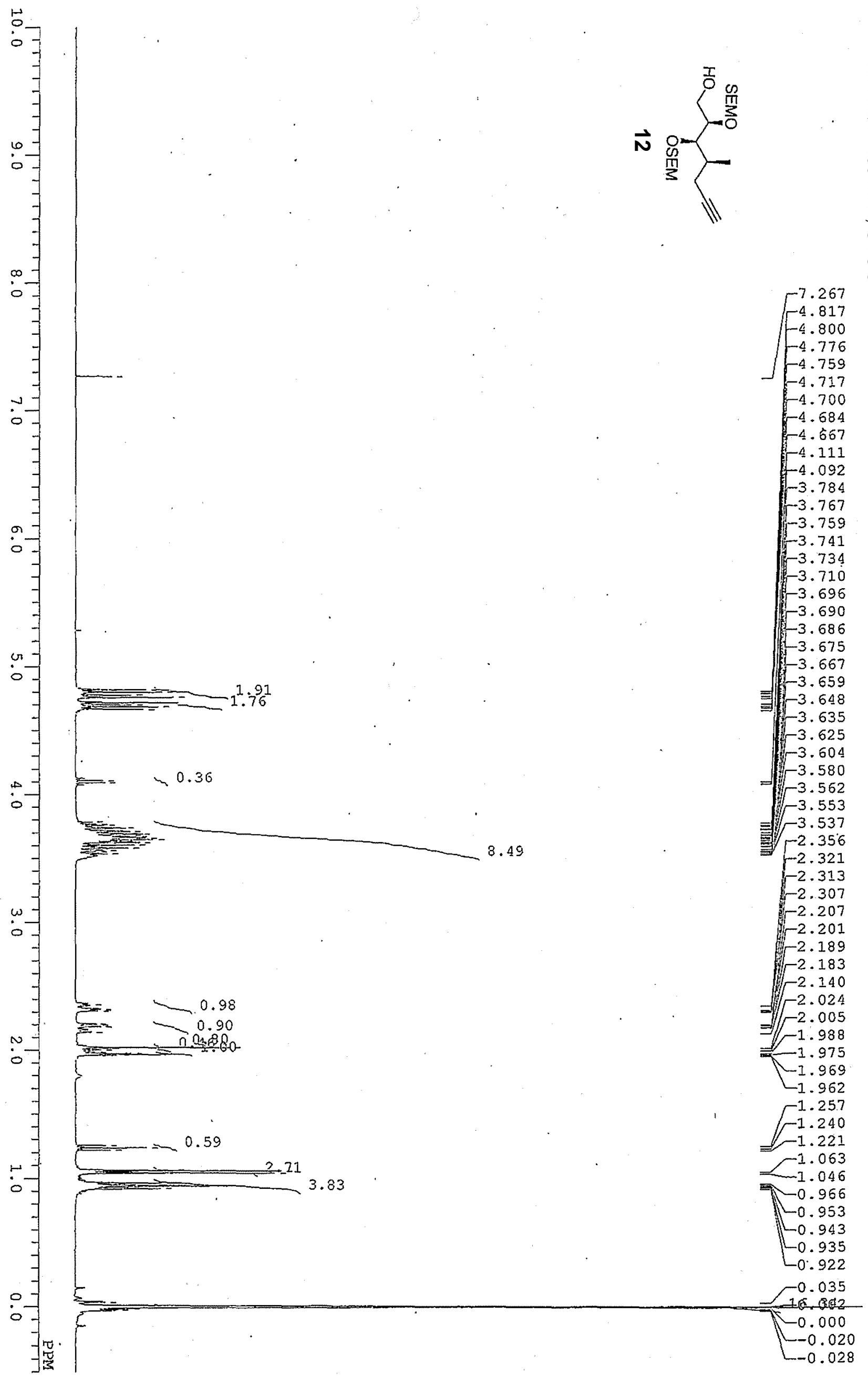




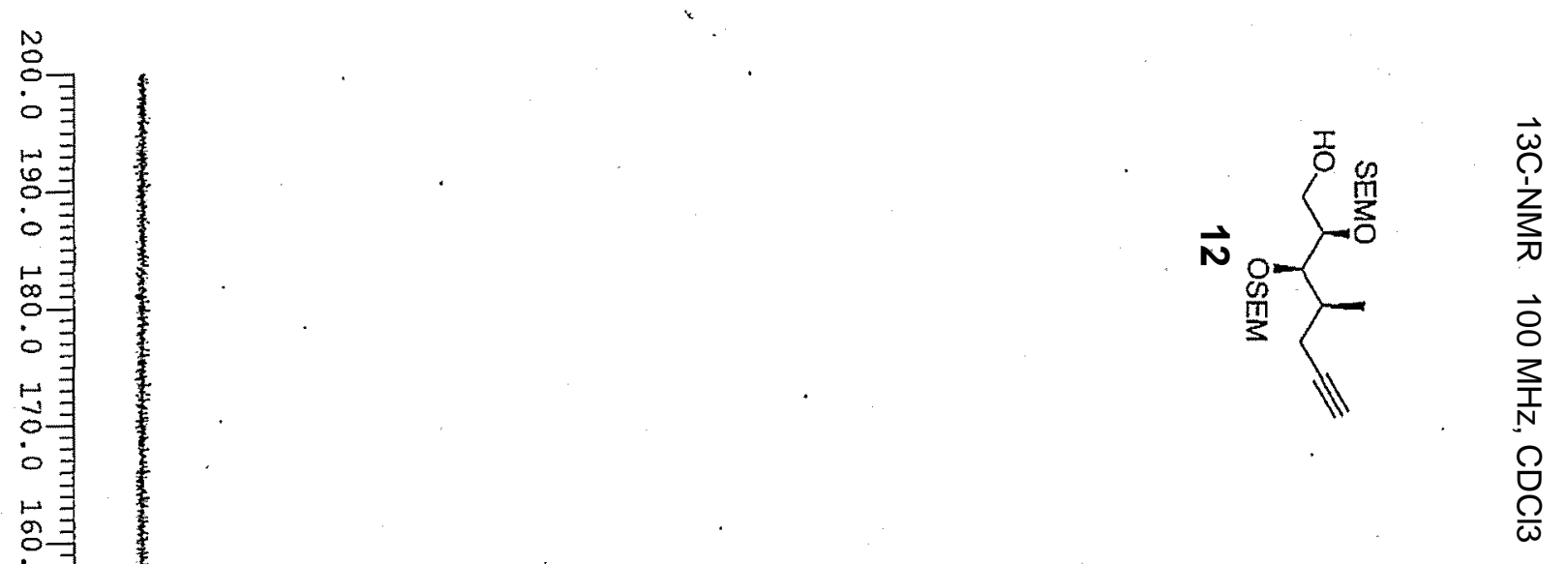

\%

0

㖨

○

o

$\circ \exists$

8.

$\therefore$

동

o

吕录

$\omega$

:

吕录
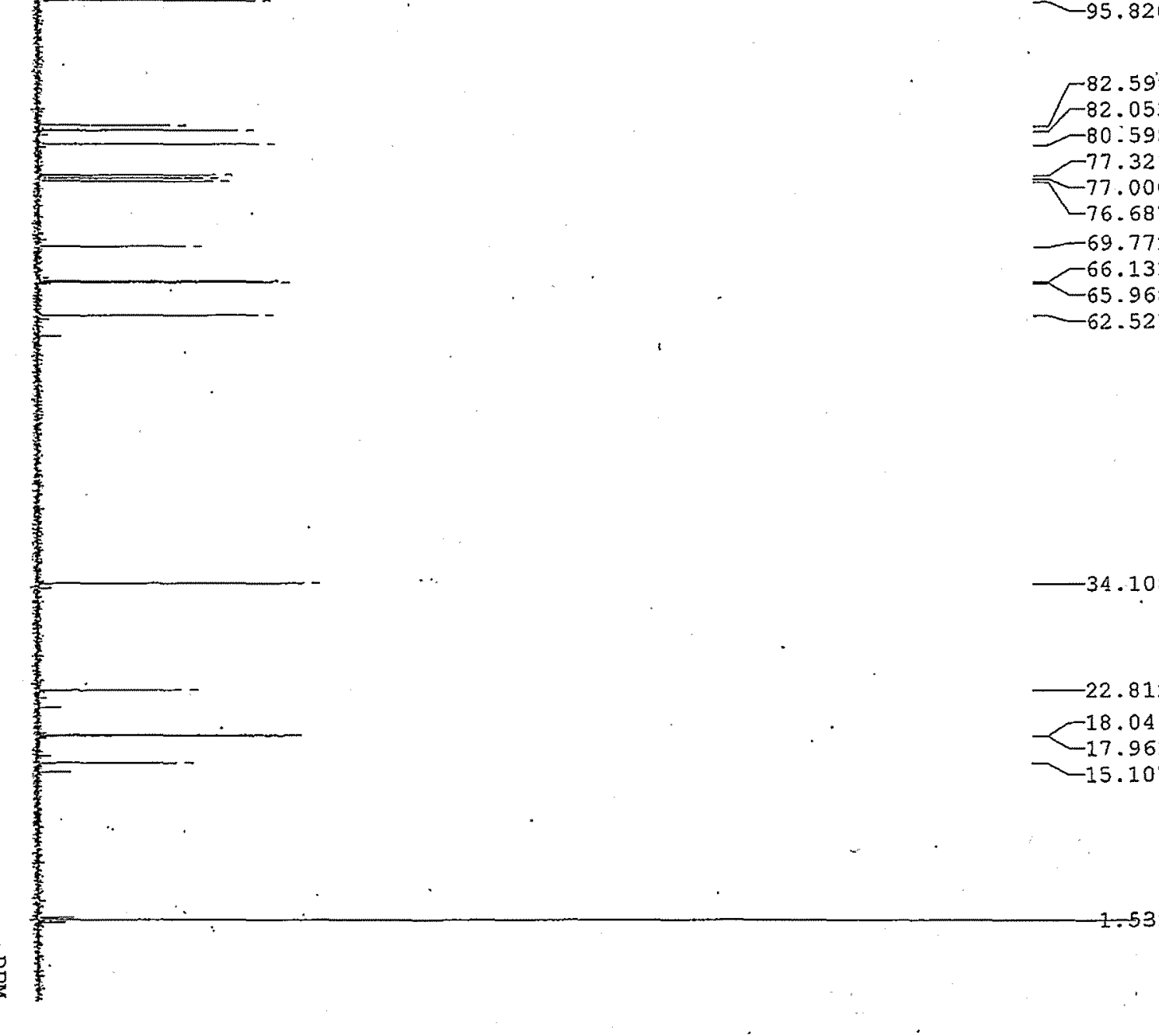

$-82.598$

$-82.055$

$-80: 598$

$-77.321$

$-77.000$

$-76.687$

$-69.772$

$-66.133$

$-65.968$

$-62.527$

占丑

o

$\circ \exists$

刍

占䏨罗

: 


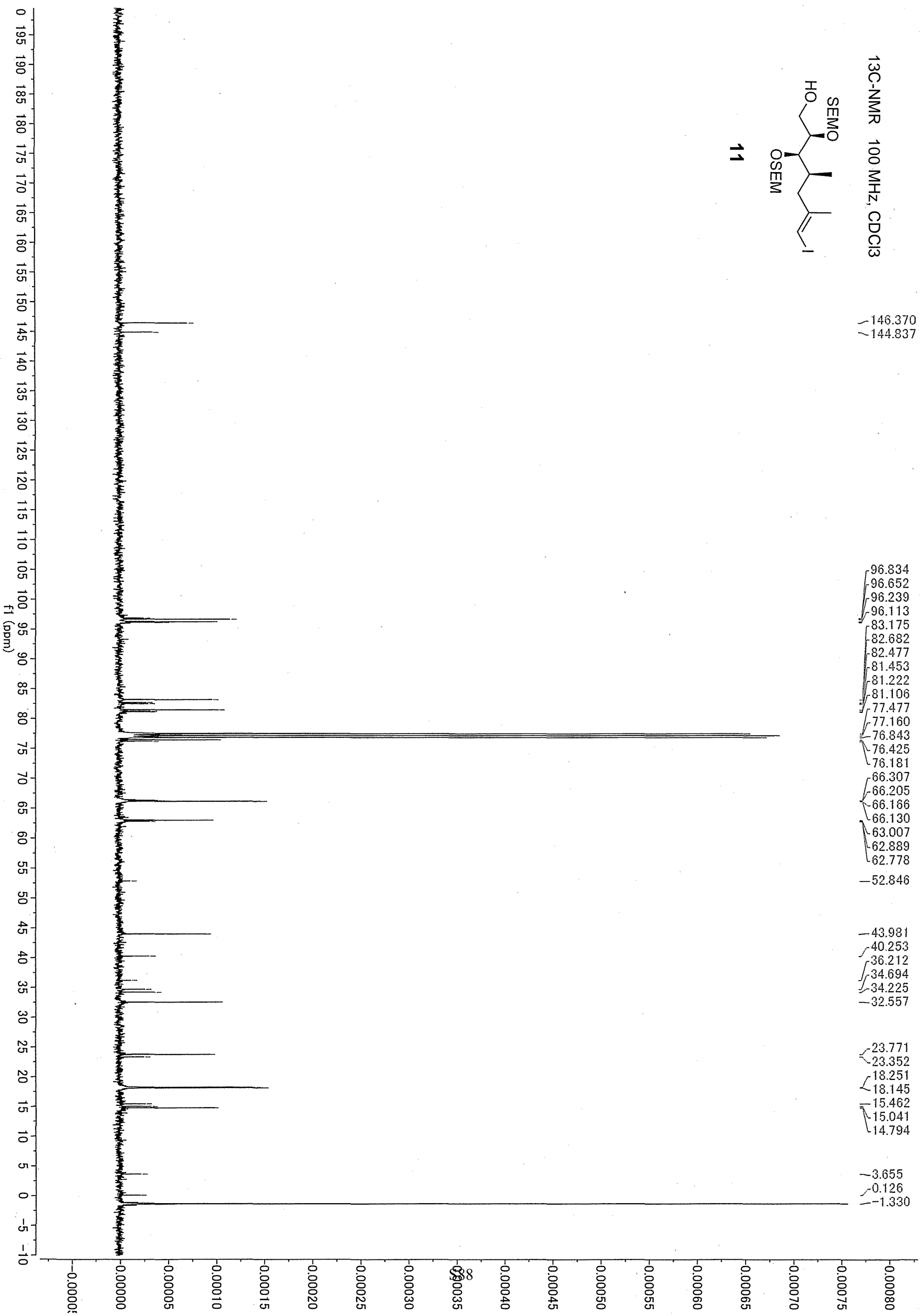




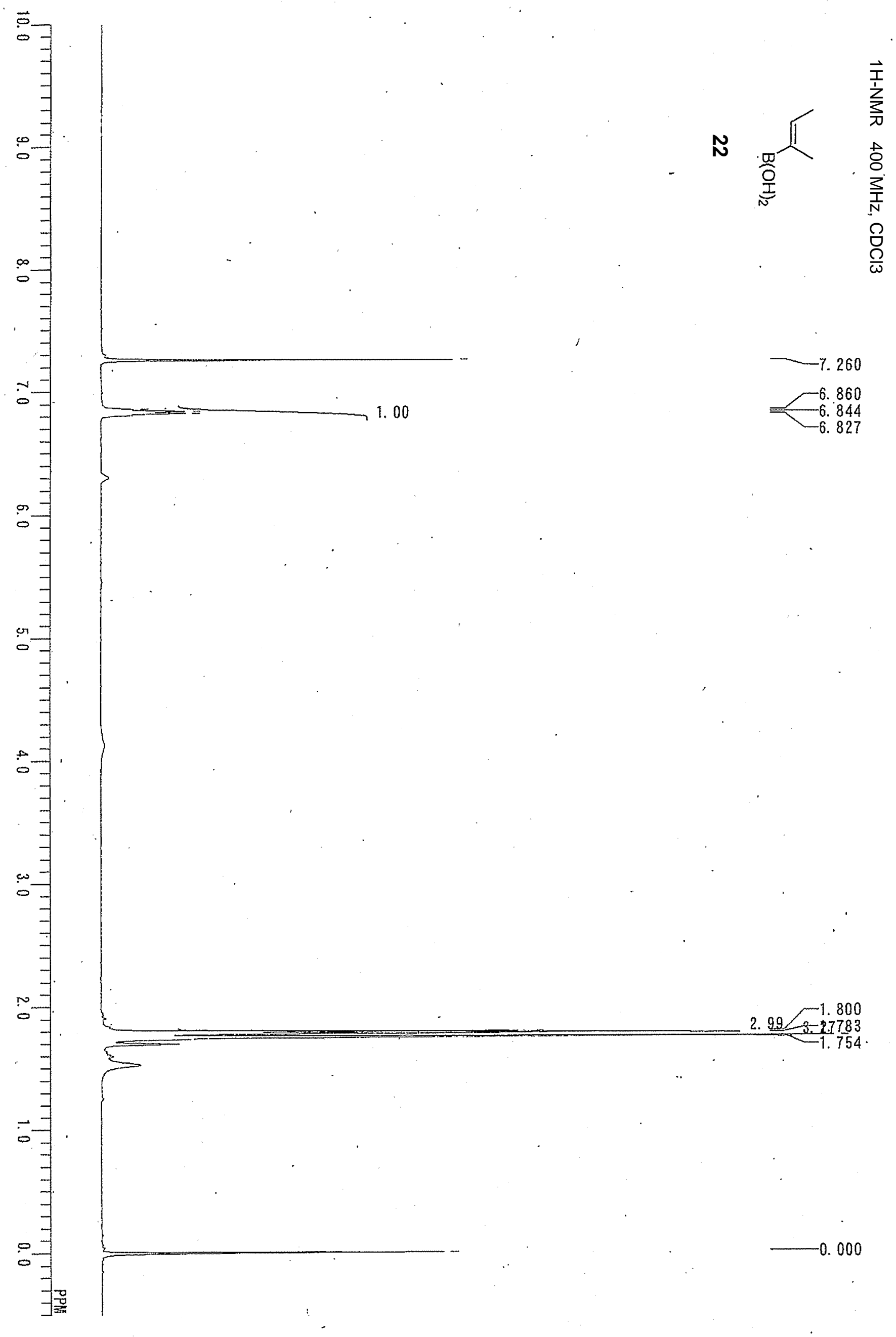




$$
1
$$




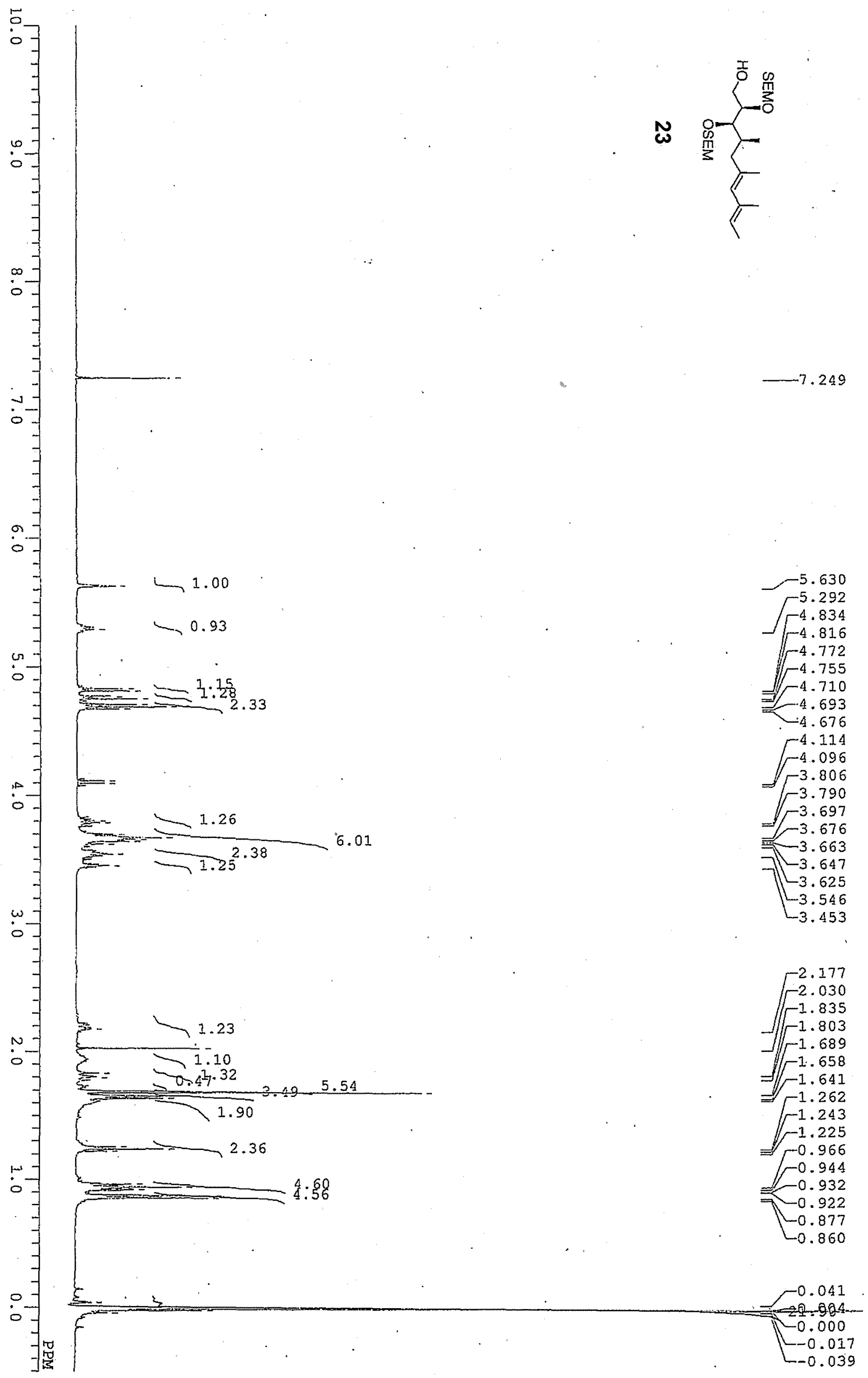




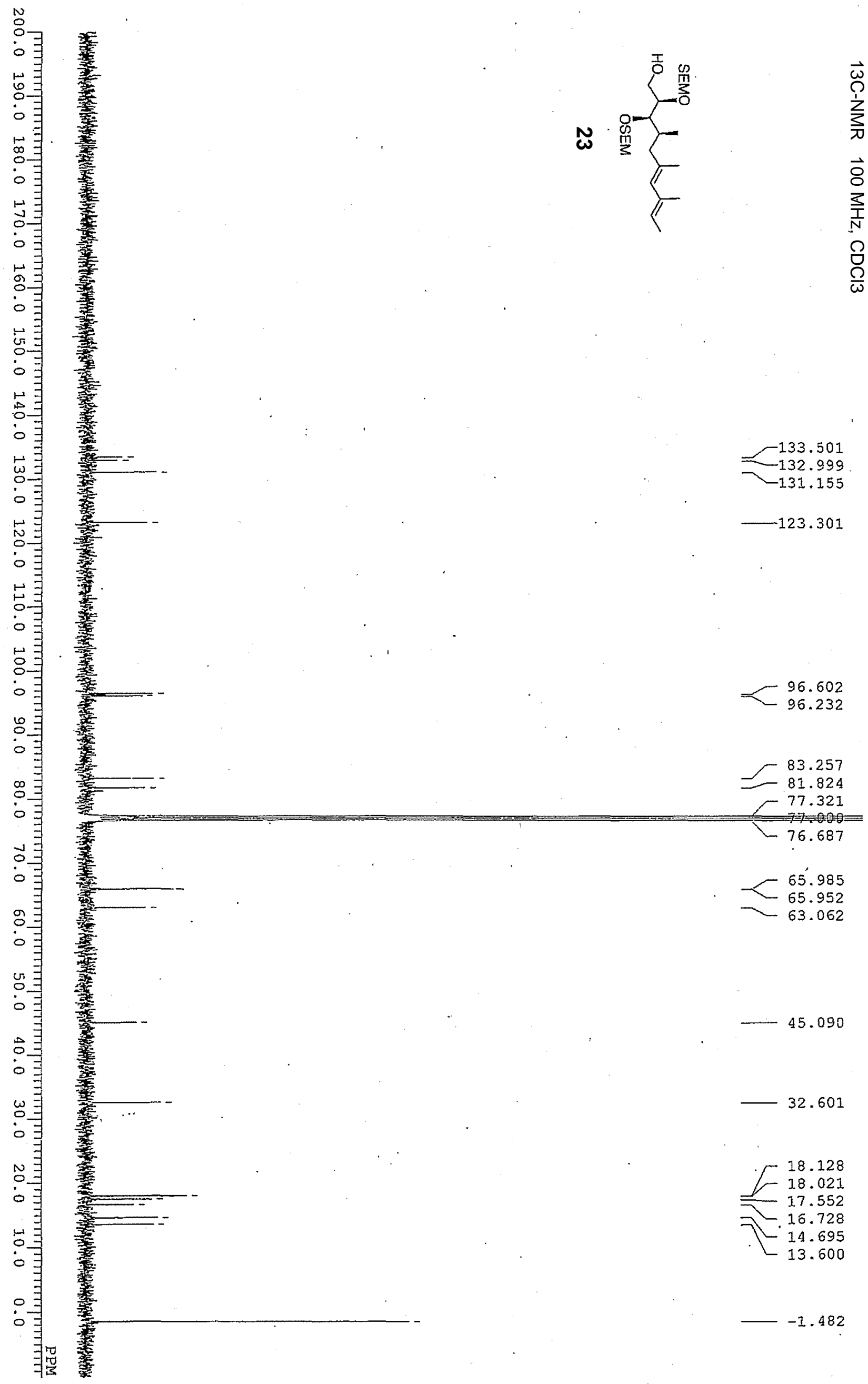




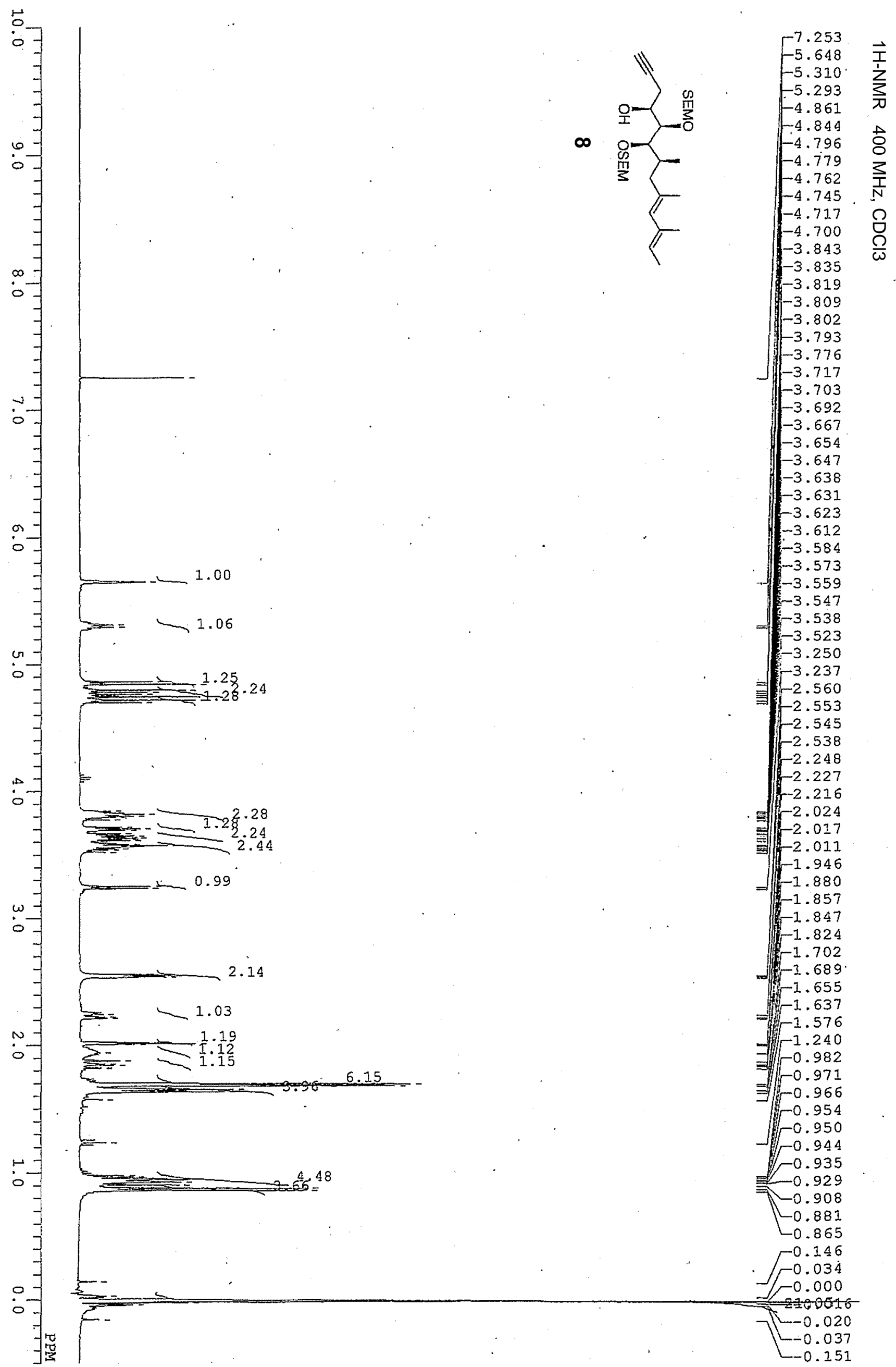




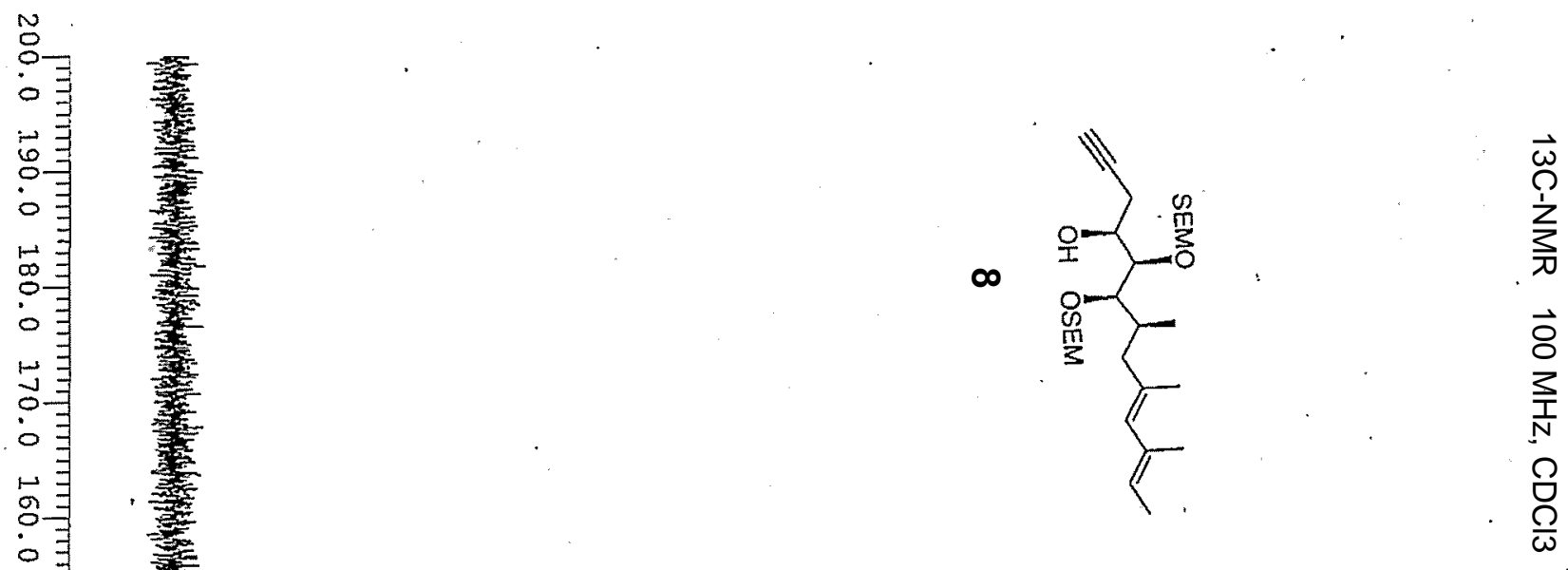

G

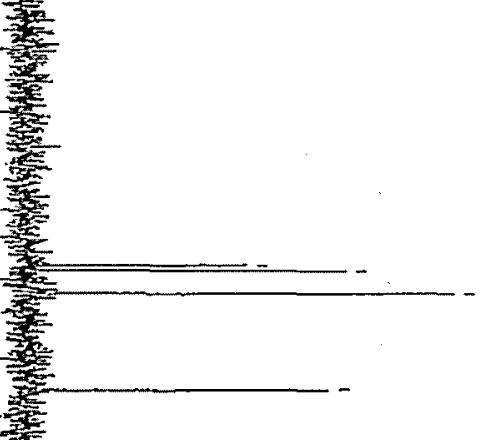

$\frac{\rho}{\omega}$

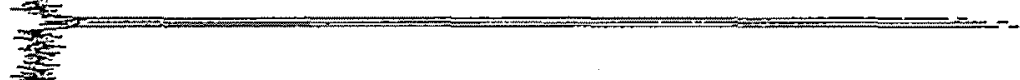




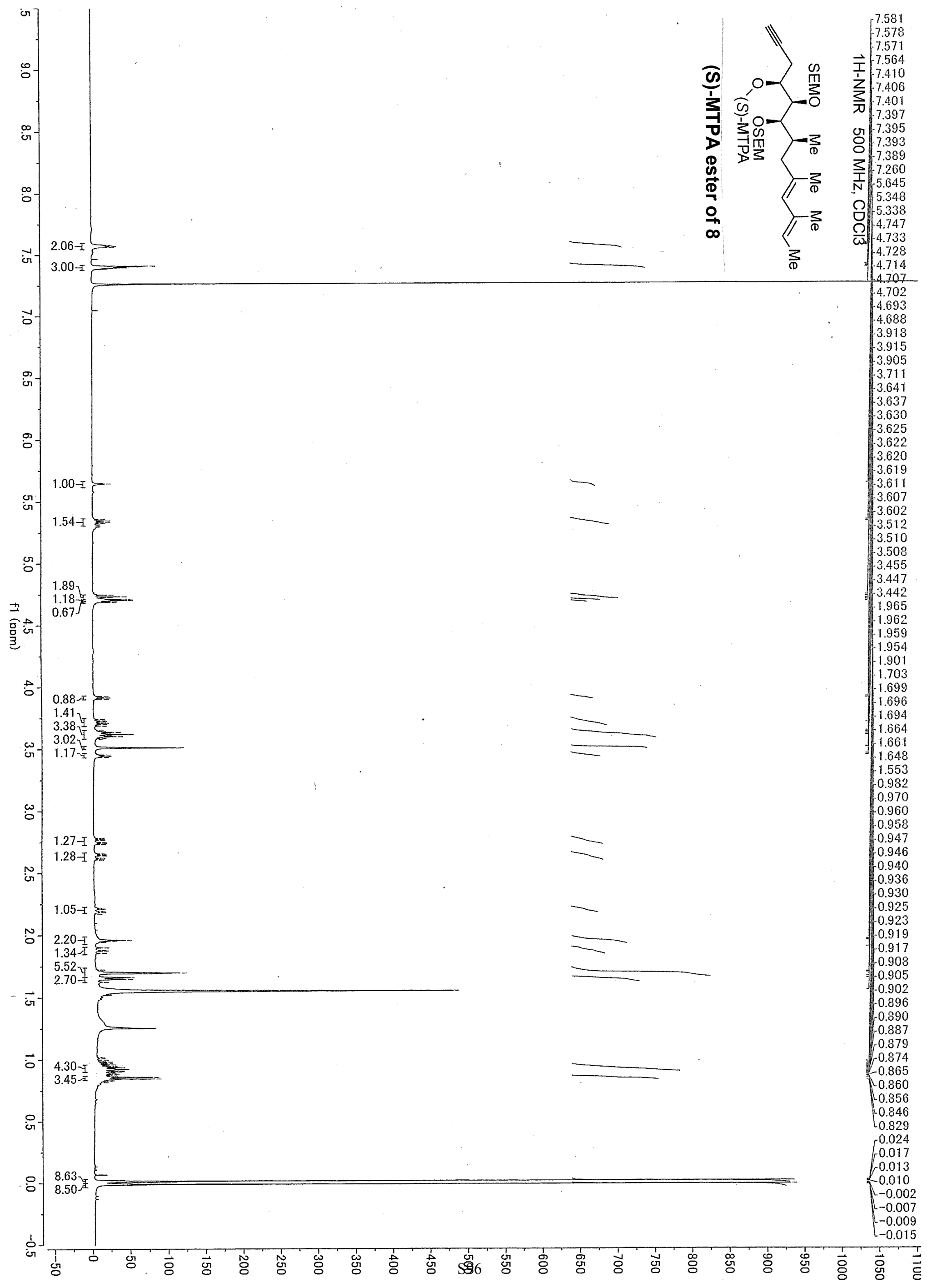




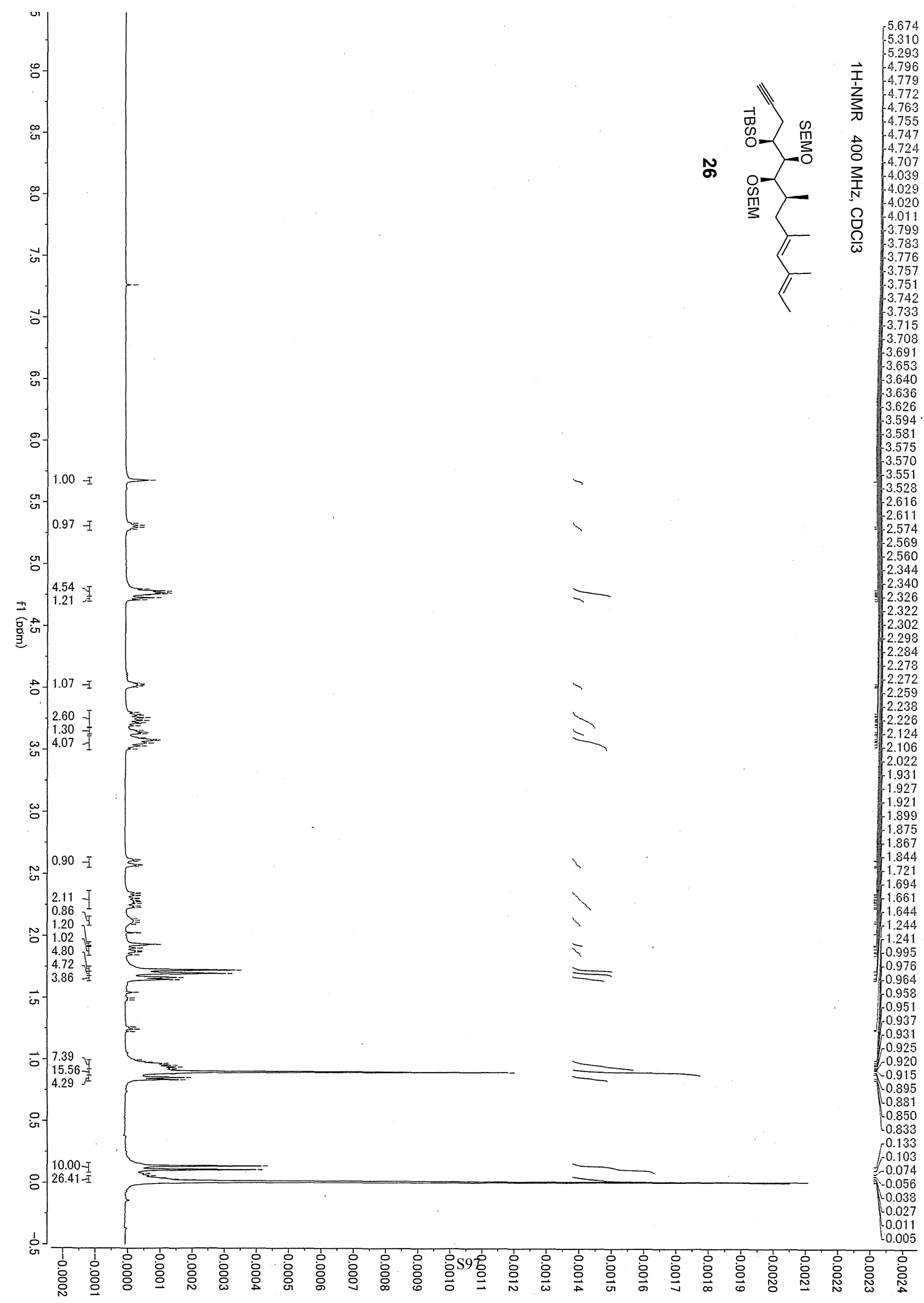




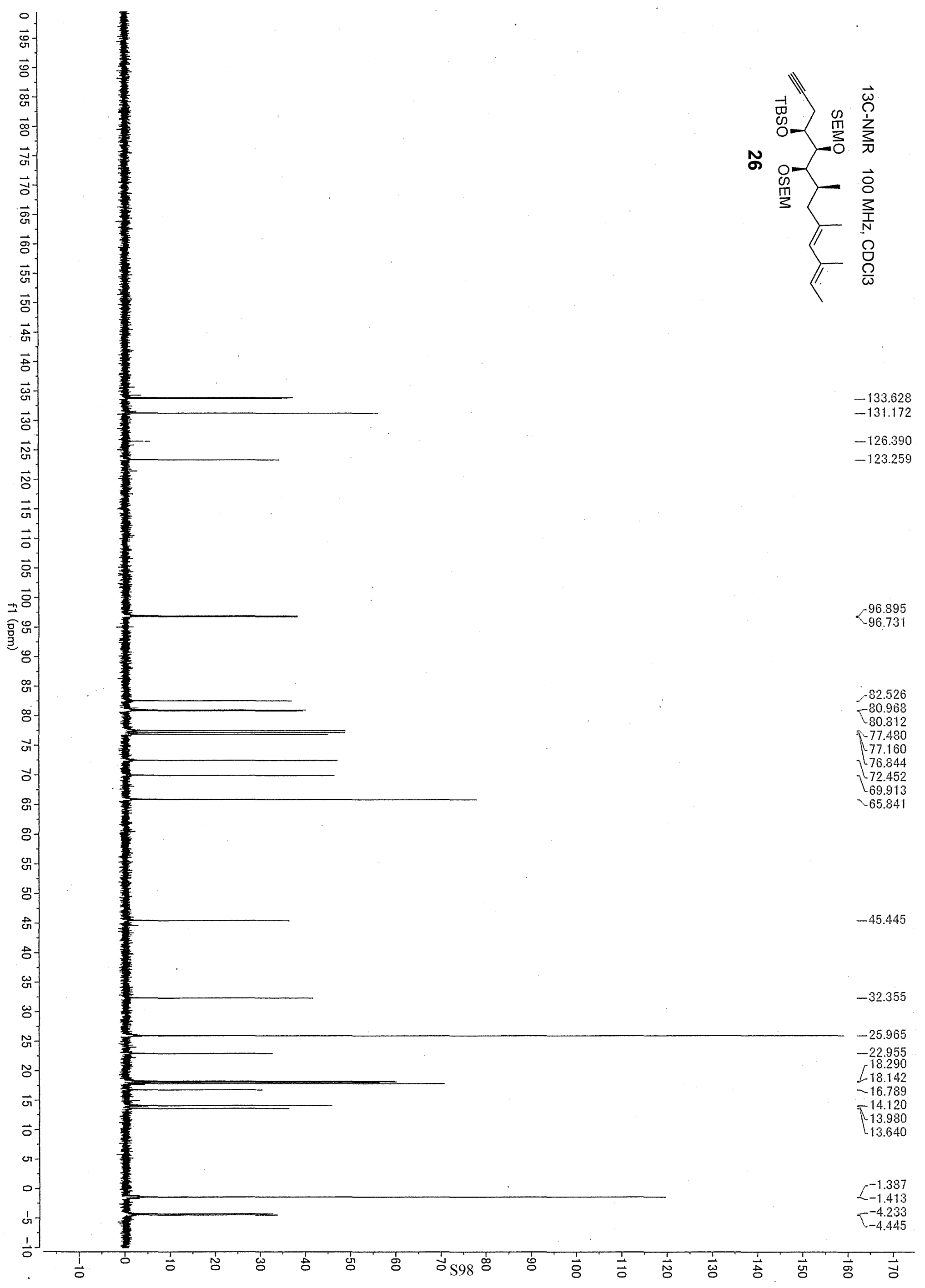




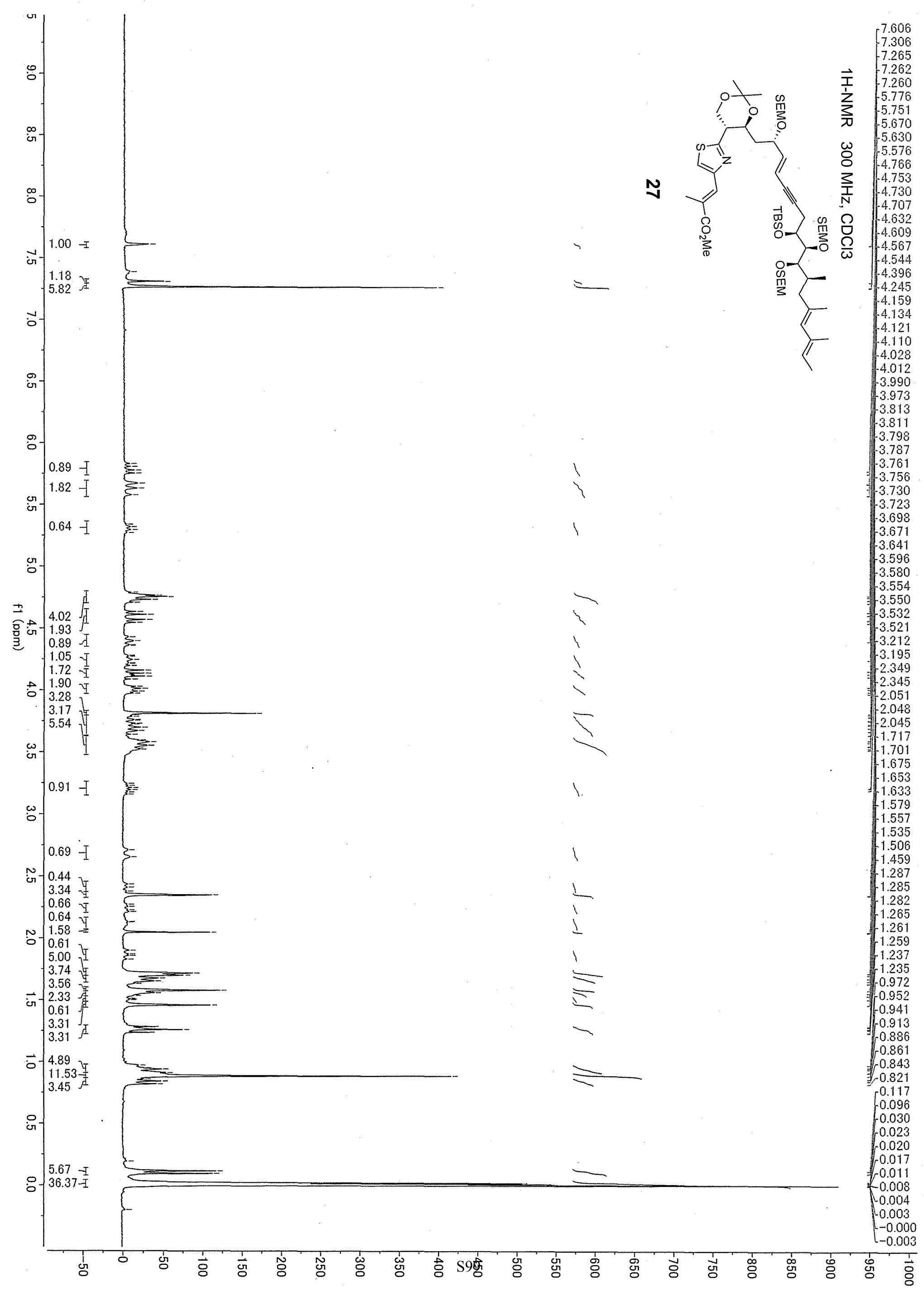




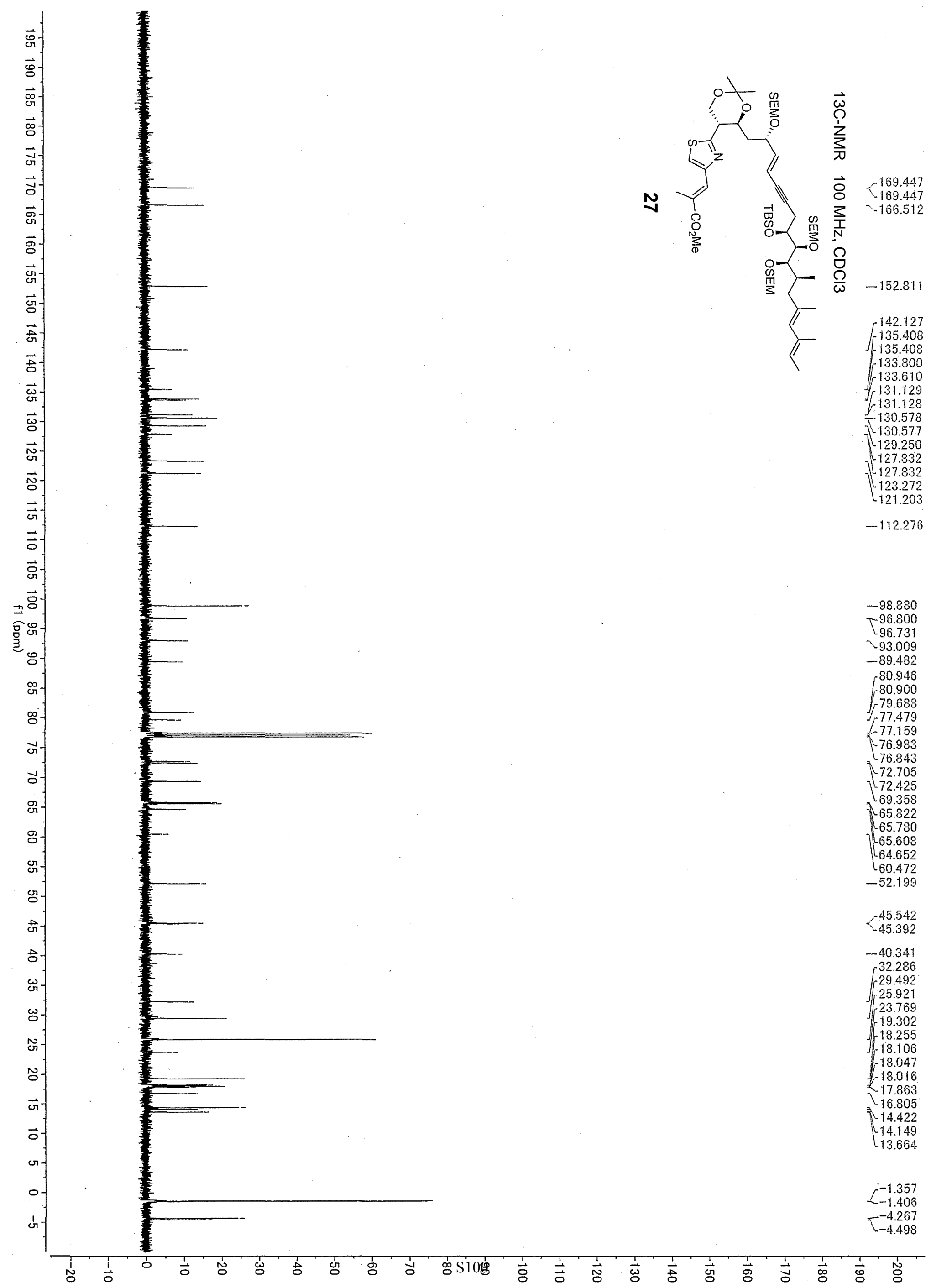




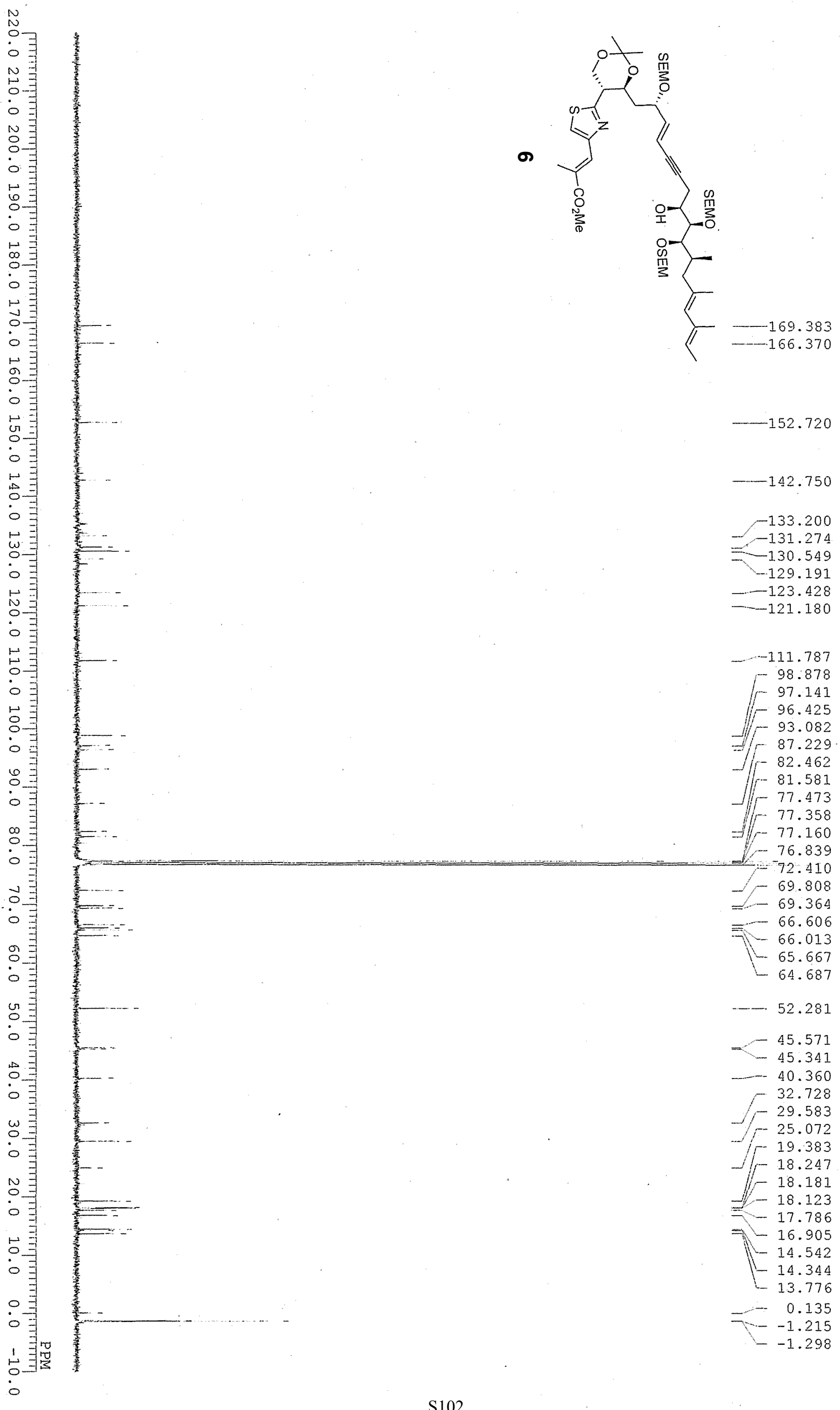




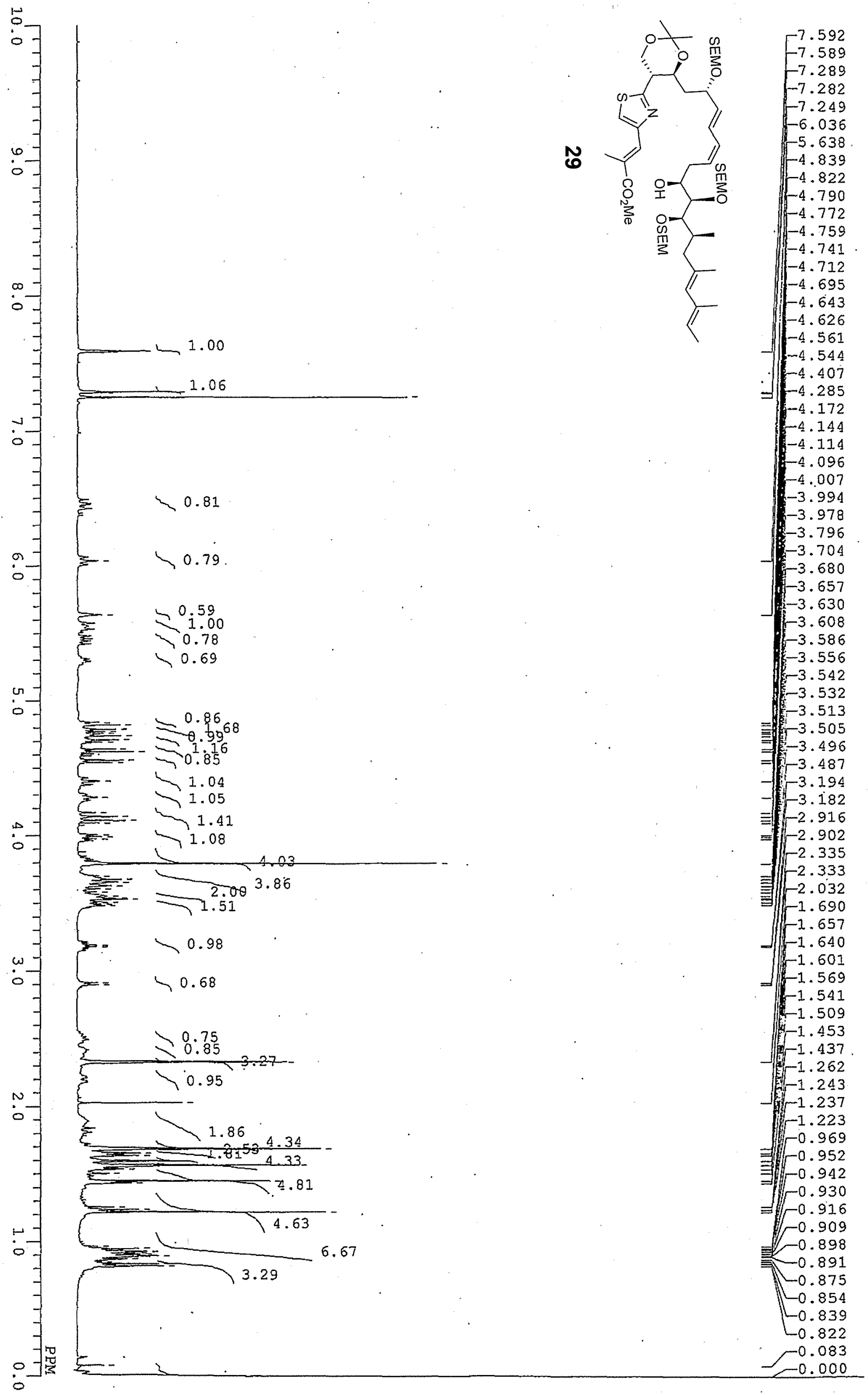

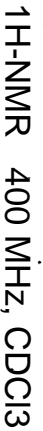



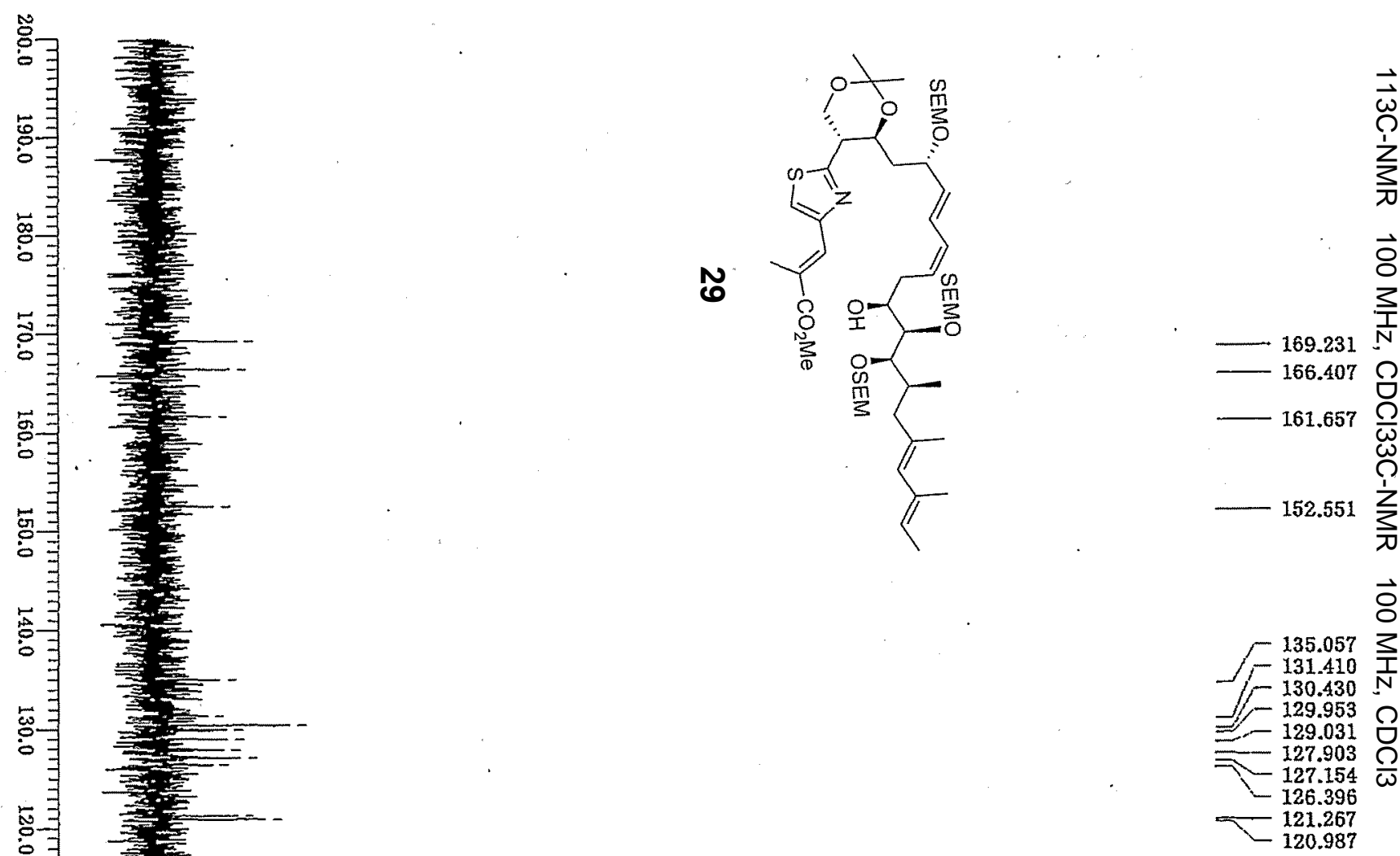

:

官

:

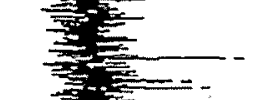

$:$

:

春

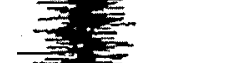

- 98.710

$\sim 96.931$

- 92.757

录

8

:

里录

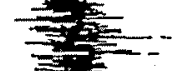

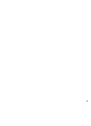

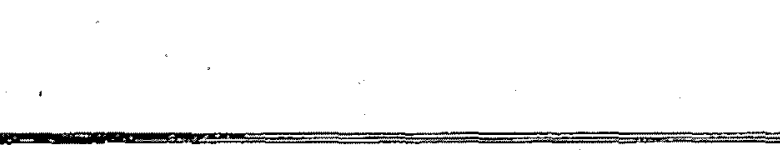

- 82.853

81.528

F 77.321 76-678 72.694

T 70.784

- 69.286

- 66.396

65.815

65.342

64.585

ㅋ․

를

ㄷ-

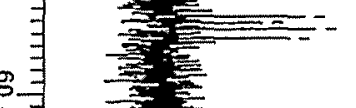

52.104

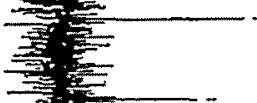

$-52.10$

$-45.526$

:

40.579

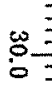

:

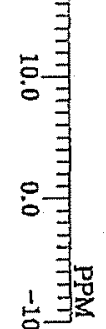

:

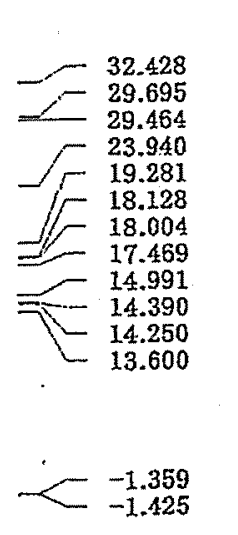




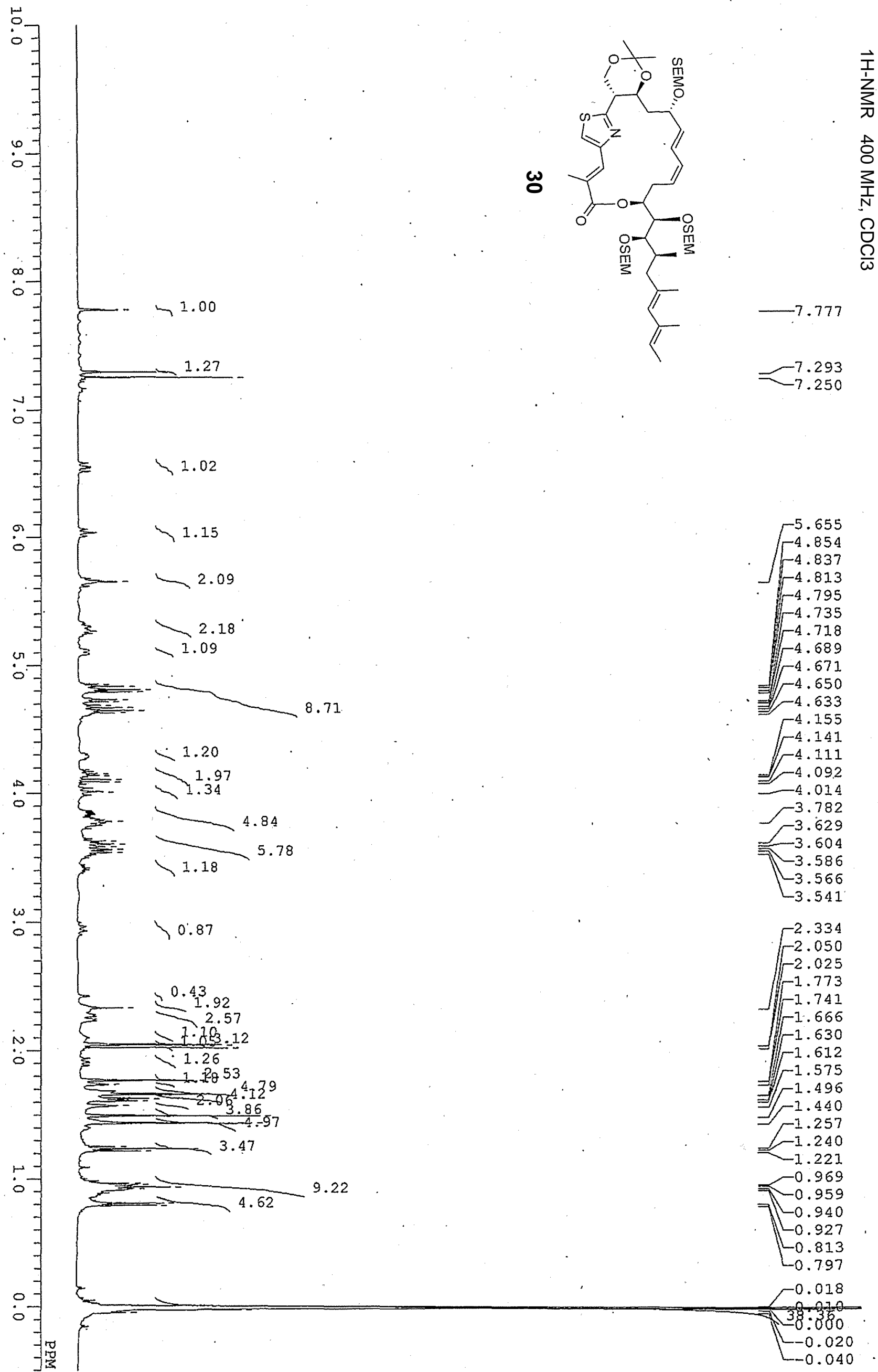



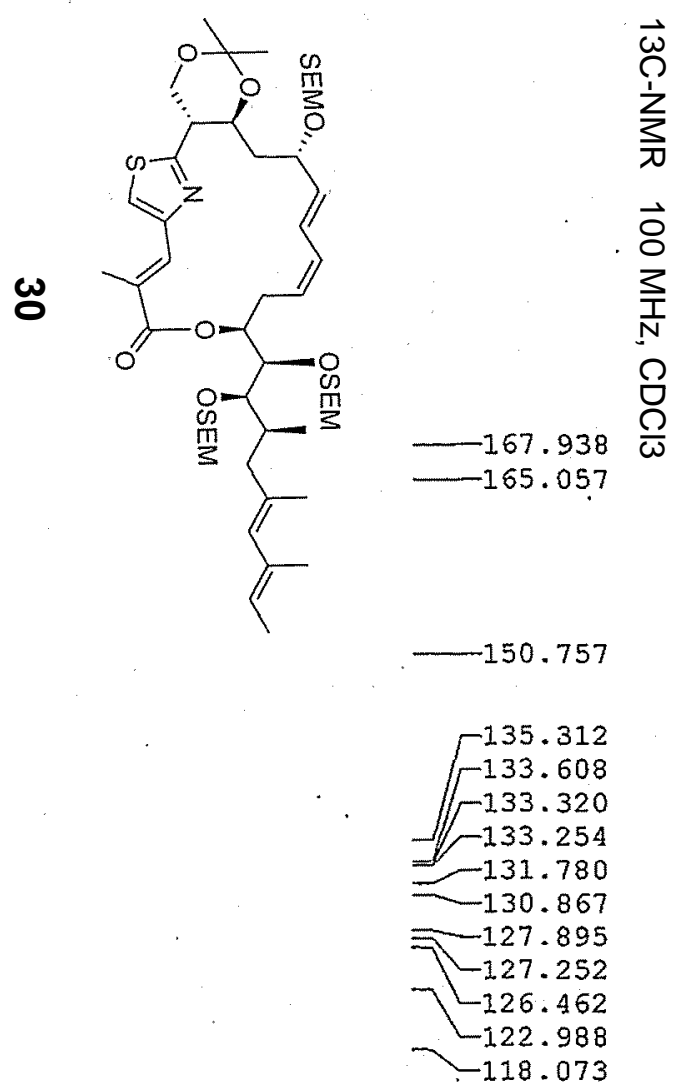

98.981

96.553

96.199

91.654

81.421

F 79.157

T 77.313

Iff 77.198

$1 / 77.000$

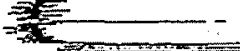

$76 \div 679$

-
- 3.592

70.875

65.943

65.672

64.980

63.581
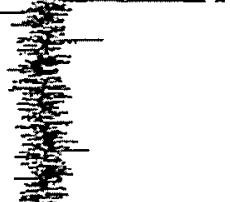

$-45.922$

43.205

38.290

$-\begin{array}{r}31.984 \\ -30.345\end{array}$

$=29.275$

- 18.984

F 18.079

$=17.766$

$-16.687$

14.160
13.814

$-13.600$

$-13.295$

$-1.342$

$-1.416$

$-1.449$ 


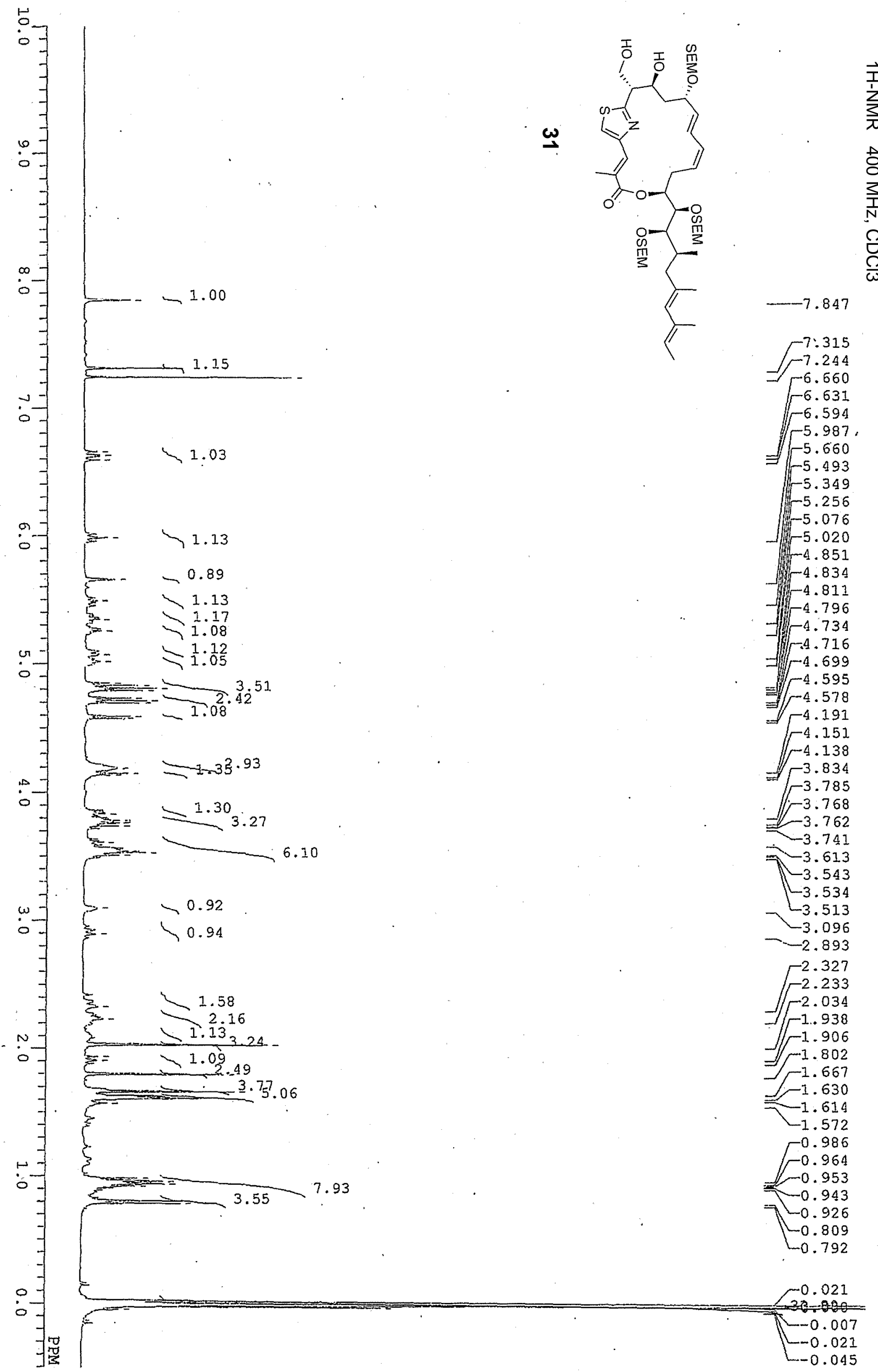


芩

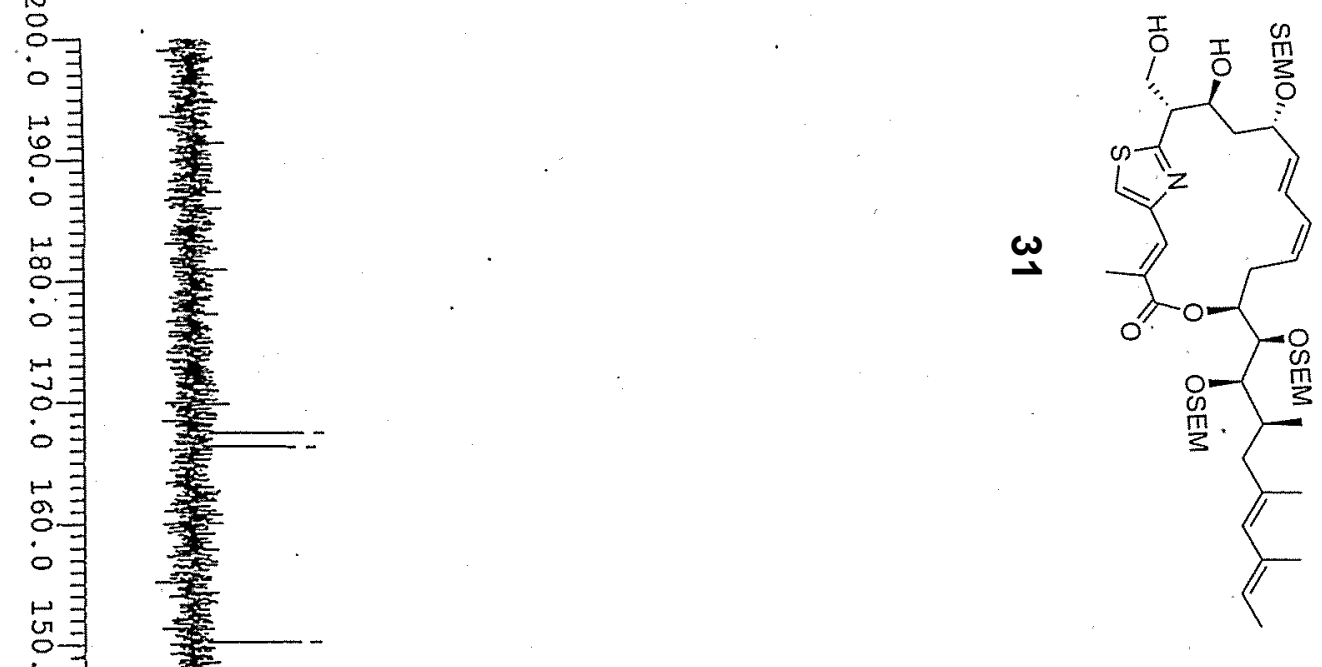

$-13.616$

$-13.345$ 


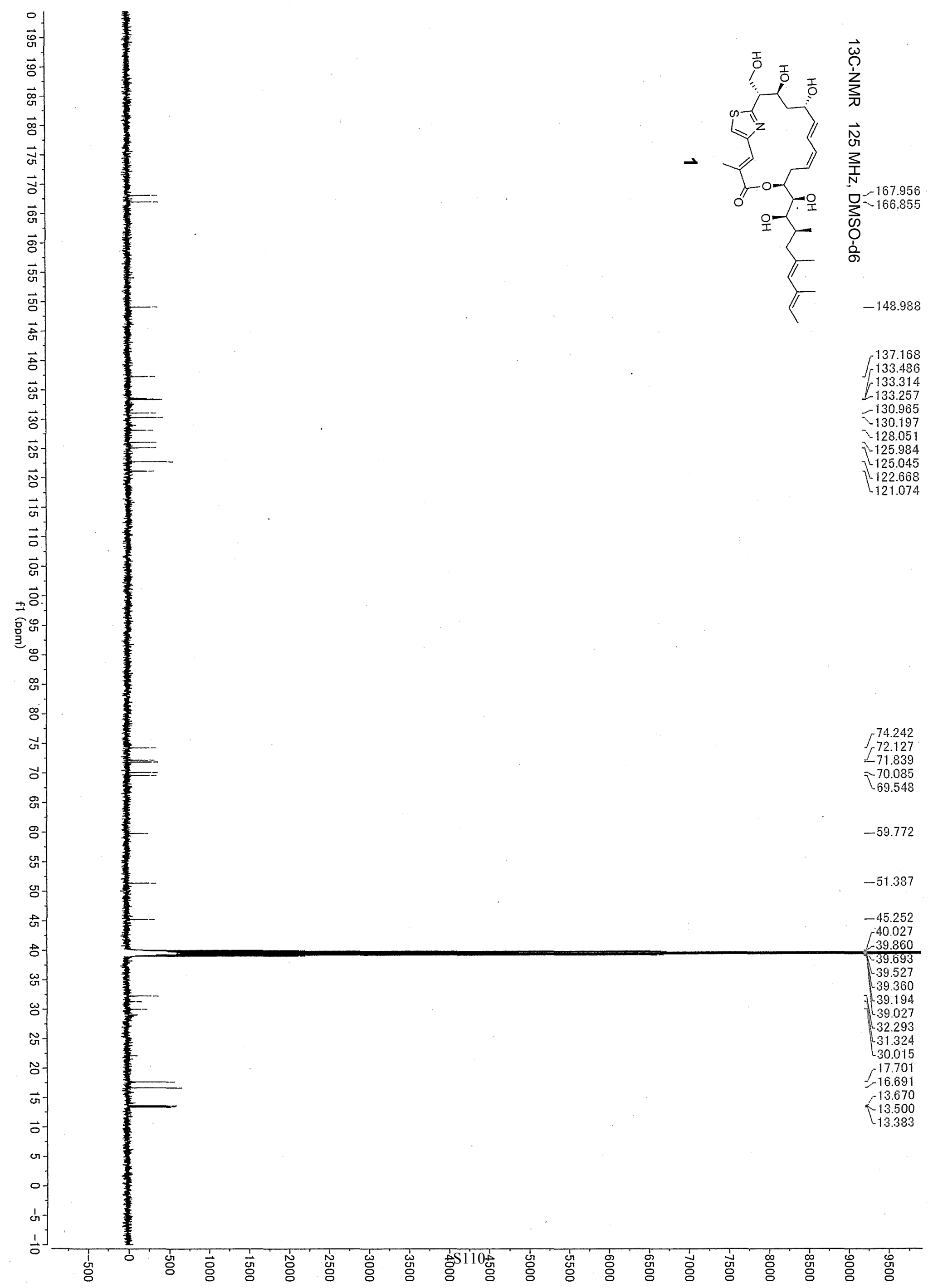

